\title{
Hydrological Modelling for Integrated Water Resources Management in a Changing Climate
}

\author{
Abbas, Salam A. A.
}

How to cite:

Abbas, Salam A. A. (2018) Hydrological Modelling for Integrated Water Resources Management in a Changing Climate. Doctoral thesis, Swansea University. http://cronfa.swan.ac.uk/Record/cronfa43733

Use policy:

This item is brought to you by Swansea University. Any person downloading material is agreeing to abide by the terms of the repository licence: copies of full text items may be used or reproduced in any format or medium, without prior permission for personal research or study, educational or non-commercial purposes only. The copyright for any work remains with the original author unless otherwise specified. The full-text must not be sold in any format or medium without the formal permission of the copyright holder. Permission for multiple reproductions should be obtained from the original author.

Authors are personally responsible for adhering to copyright and publisher restrictions when uploading content to the repository.

Please link to the metadata record in the Swansea University repository, Cronfa (link given in the citation reference above.)

http://www.swansea.ac.uk/library/researchsupport/ris-support/ 


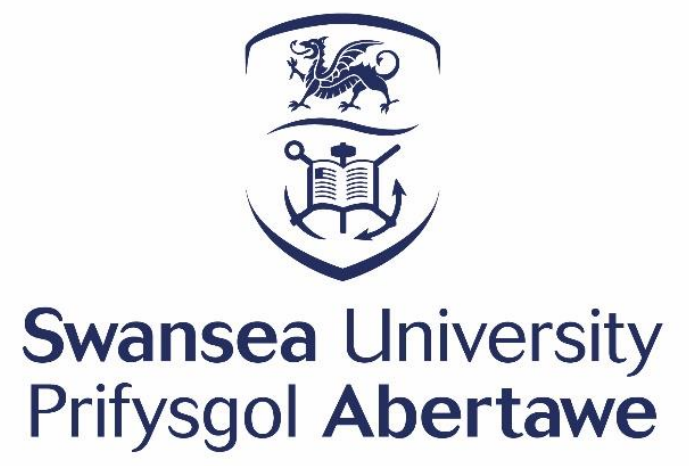

\section{Hydrological Modelling for Integrated Water Resources Management in a Changing Climate}

\section{Salam Abd Albare Abbas}

Submitted to Swansea University in fulfilment of the requirements for the degree of Doctor of Philosophy in the College of Engineering

SWANSEA UNIVERSITY 


\begin{abstract}
Hydrological models are a simplified representation of hydrological processes and can be very used for the water resources assessment and gain an integral view of the water resources status for integrated water resources management IWRM. Furthermore, they can be used to investigate the possible impacts and trends resulting from different types of scenarios, such as climate change impact studies.
\end{abstract}

Accordingly, with IWRM as the future application, the primary objectives of this study is to use a hydrological model, SWAT for the modelling of a highly-regulated river basin through the physical flow control (reservoirs release in the upstream region), the Dee River Watershed in the United Kingdom. Moreover, an essential aspect of model input uncertainty, i.e. precipitation is investigated on the simulated streamflow where different methods of rainfall pre-processing are used. Furthermore, a quantile regression method is employed for analysing the long-term historical trend of rainfall, river flow and catchment water yields focusing on the patterns of the data close to 'extreme' regimes, to link them to the events of interests for the climate change impact studies.

Additionally, a reliable simulation of both land surface and groundwater hydrological processes is a far important step for IWRM. One way to achieve such purpose is the coupling of surface and groundwater models. The land surface model (SWAT) is coupled with the groundwater flow model (MODFLOW) to improve the baseflow simulation of the SWAT standalone in the study area. Another critical aspect of this study is the investigation of parameter uncertainty of the coupled SWATMODFLOW. Finally, the climate projection data from the CMIP5 project is utilised with allocation model, Water Evaluation and Planning software WEAP to address climate change impact for future scenarios on water resources.

All presented models performed well in demonstrating the study conditions, as indicated by the statistical performance. The research approach of the integrated models can generally apply to any catchment and inspired by the need of considering all aspects related to hydrological models for IWRM to bridge the gap of between stakeholder involvement and natural hydrological processes in building and applying integrated models to ensure acceptability and application in decision-making for IWRM. 


\section{Declaration and Statements}

I, Salam Abd Albare Abbas, declare that this thesis titled, 'Hydrological Modelling for Integrated Water Resources Management in a Changing Climate' and the work presented in it are my own, I confirmed that. This work has not previously been accepted in substance for any degree and is not being concurrently submitted in candidature for any degree.

Singed. (Salam Abd Albare Abbas)

Date.

This thesis is the result of my own investigations, except where otherwise stated. Where correction services have been used, the extent and nature of the correction is clearly marked in a footnote(s).

Other sources are acknowledged by footnotes giving explicit references. A bibliography is appended.

Signed. (Salam Abd Albare Abbas)

Date.

I hereby give consent for my thesis, if accepted, to be available for photocopying and for inter-library loan, and for the title and summary to be made available to outside organisations.

Signed. (Salam Abd Albare Abbas)

Date. 


\section{Contents}

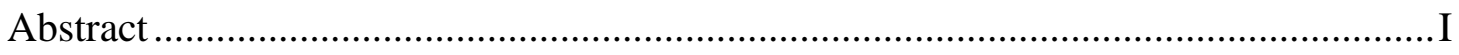

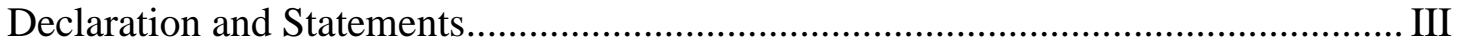

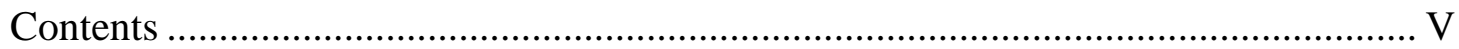

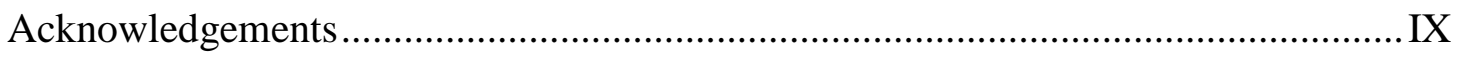

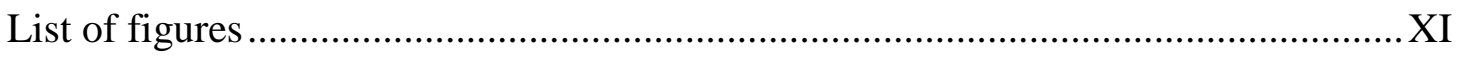

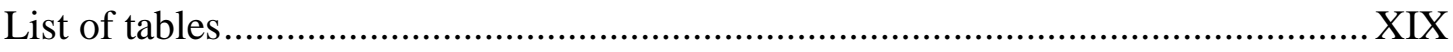

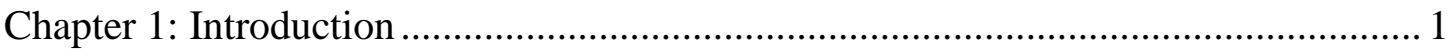

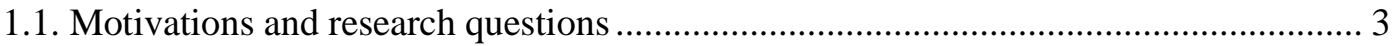

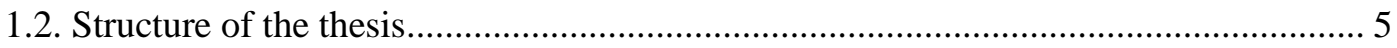

Chapter 2: A Review on Modelling Support for Water Resources Management.......... 7

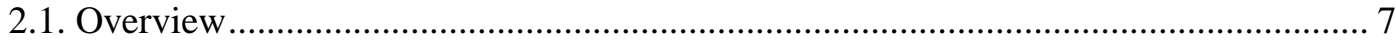

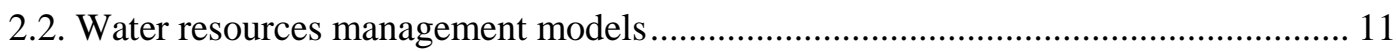

2.2.1. Allocation models .......................................................................................... 12

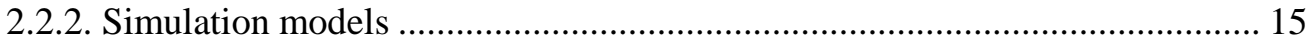

2.2.3. Hydrological models ................................................................................. 18

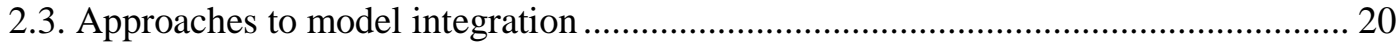

2.3.1. Integrated models versus supermodels ........................................................ 22

2.3.2. Model integration at various levels .............................................................. 23

2.3.3. Scaling and uncertainty issues in implementation ....................................... 25

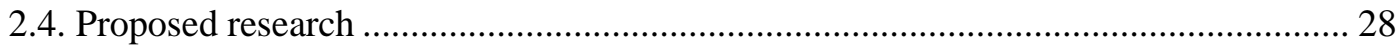

Chapter 3: Hydrological Modelling of a Highly-regulated River Basin...................... 31

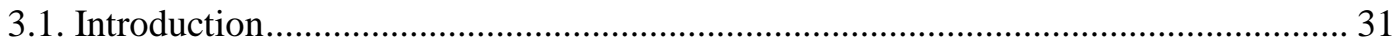

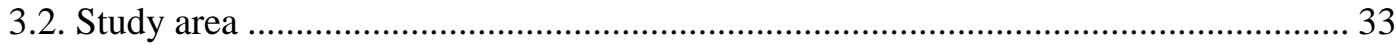

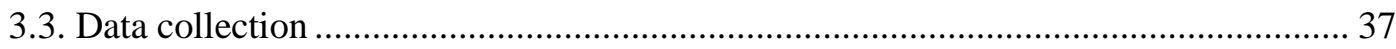

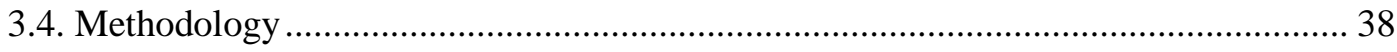

3.4.1. Flow regulation- water abstraction interaction ............................................. 39

3.4.2. Modelling streamflow using SWAT .............................................................. 42

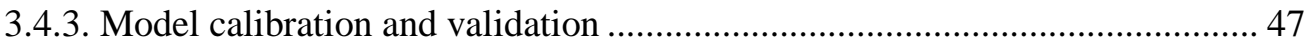

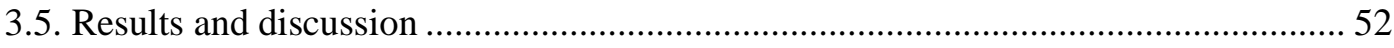

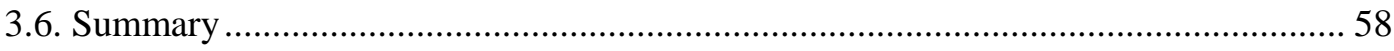

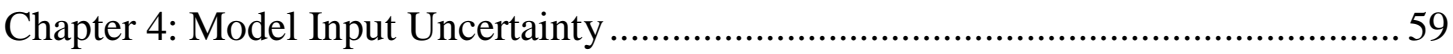

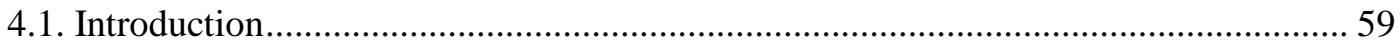


4.2. The CEH-GEAR precipitation dataset. 65

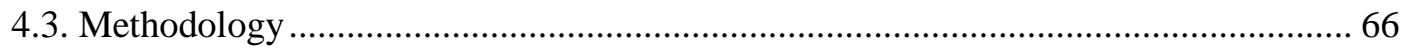

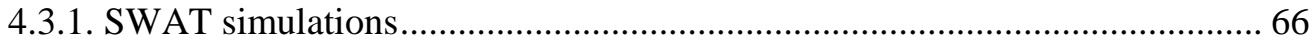

4.3.2. Precipitation pre-processing methods ............................................................. 68

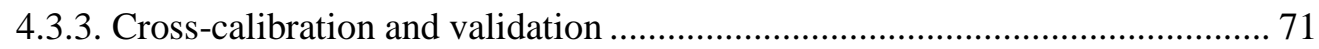

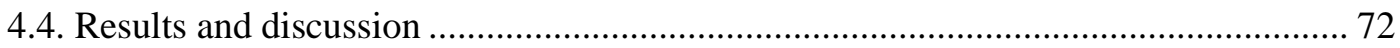

4.4.1. Comparison of point/grid precipitation and spatial distributions................... 72

4.4.2. Impacts of rainfall pre-processing on model simulations ............................... 77

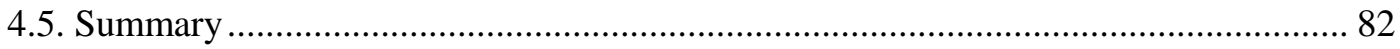

Chapter 5: Trend Analysis of Regional Water Resources ........................................ 85

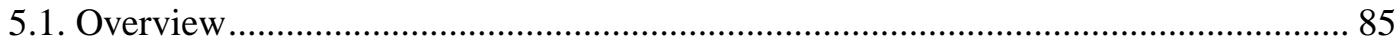

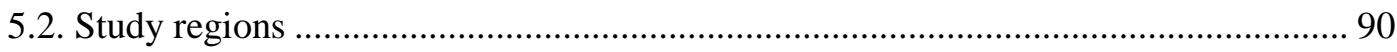

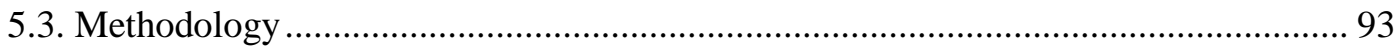

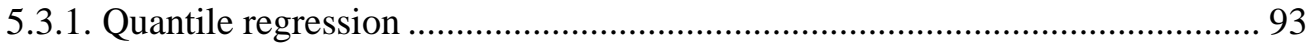

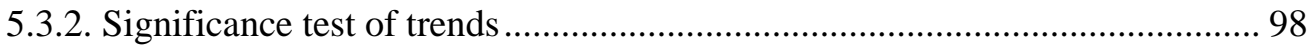

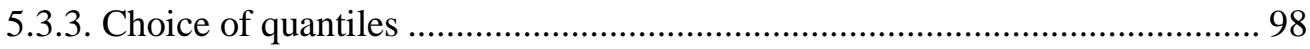

5.3.4. Standardised Precipitation Index (SPI) ………….......................................... 99

5.3.5. Extreme precipitation indices ....................................................................... 101

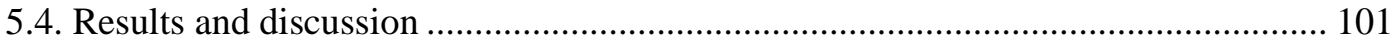

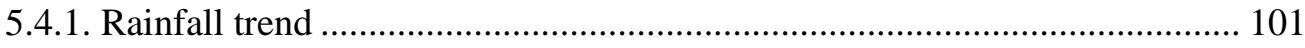

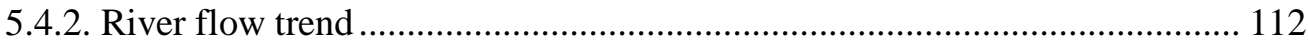

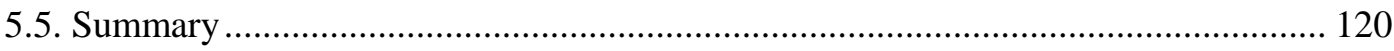

Chapter 6: Coupled Surface-Groundwater Modelling ............................................. 123

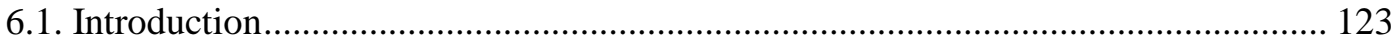

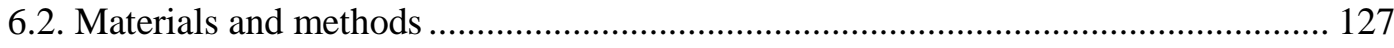

6.2.1. Groundwater data.................................................................................. 127

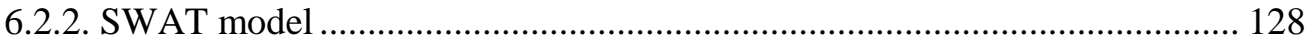

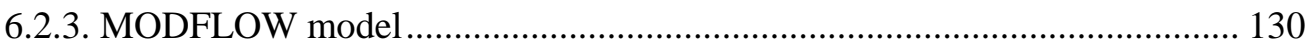

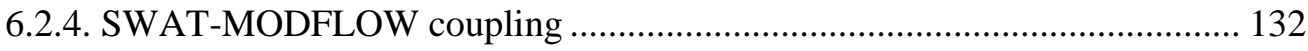

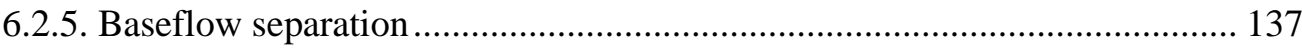

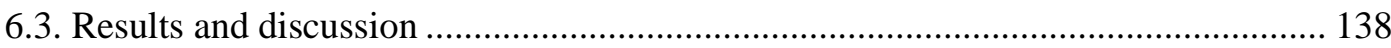

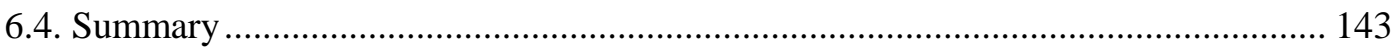

Chapter 7: Uncertainty Analysis of the Coupled Surface-Groundwater Models ...... 144

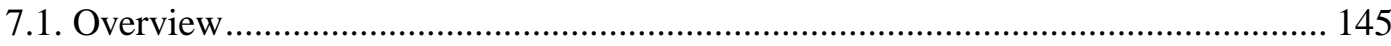

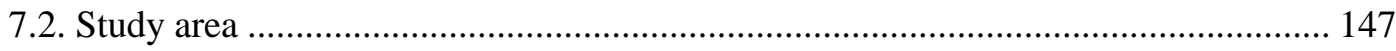


7.3. Sensitivity Analysis 147

7.4. Global Sensitivity Analysis (GSA) techniques .......................................................... 149

7.4.1. Elementary Effect Test (EET) ............................................................... 149

7.4.2. Generalised Likelihood Uncertainty Estimation (GLUE)............................. 151

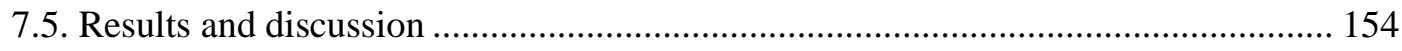

7.5.1. Uncertainty of parameters.......................................................................... 154

7.5.2. Parameters uncertainty using the EET method ............................................ 159

7.5.3. Parameters uncertainty using GLUE method.............................................. 161

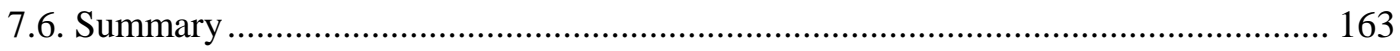

Chapter 8: The Reliability of Climate Projections in the Concept of Driving Models 165

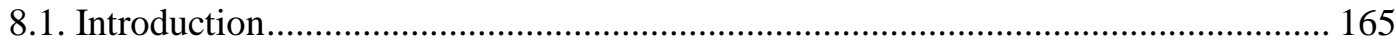

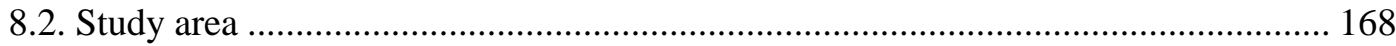

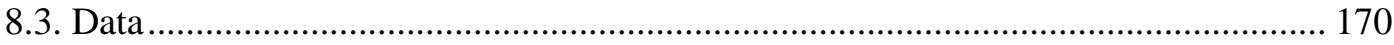

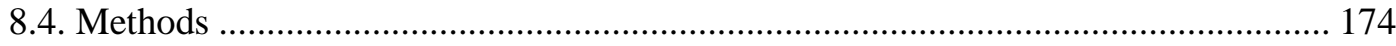

8.4.1. The goodness of fit tests (GOF) .............................................................. 174

8.4.2. Bias correction: quantile mapping .............................................................. 176

8.4.3. Mann Kendall trend test (MK) .................................................................. 177

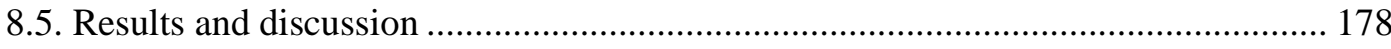

8.5.1. Statistical comparison of the observed and the modelled precipitations ...... 178

8.5.2. Trend analysis of average rainfall.............................................................. 183

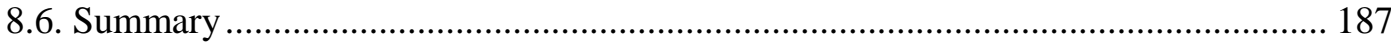

Chapter 9 Climate Change Impact on Water Resources.......................................... 189

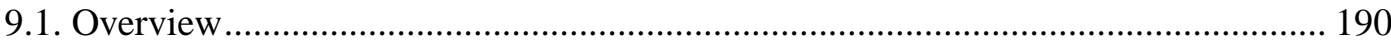

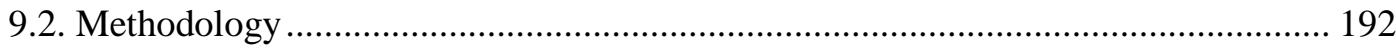

9.2.1. Hydrological simulation.............................................................................. 192

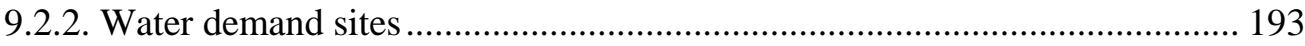

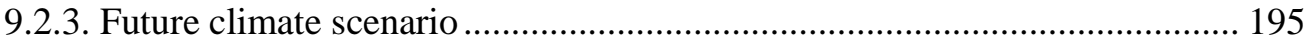

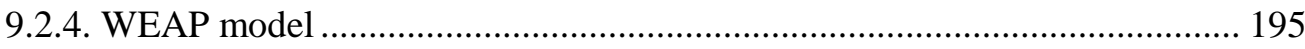

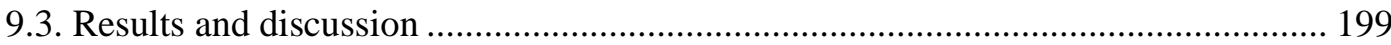

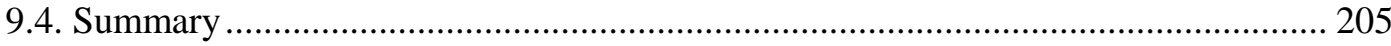

Chapter 10: Conclusions and Recommendations .................................................. 206

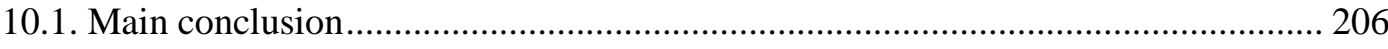

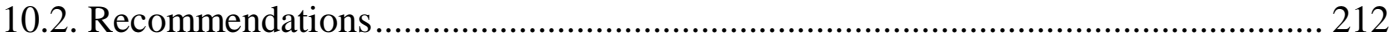

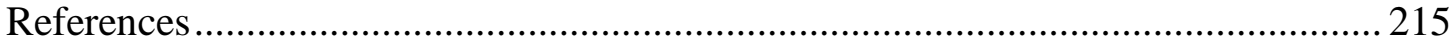




\section{Acknowledgements}

First and foremost, I would like to thank God Almighty for giving me the ability, strength, knowledge and opportunity to undertake this research study and to persevere and complete it satisfactorily. Without his blessings, this achievement would not have been possible.

I would like to dedicate this thesis to my grandfather who, although no longer with us, from an early age, inspired me to pursue my interests in science, for his love and encouragement in all my endeavours and the value, he placed on family. I would like to thank my mom and dad. Your devotion, unconditional love and support, a sense of humour, patience, optimism and advice was more valuable than you could ever imagine. I appreciate all your patience and support during my $\mathrm{PhD}$ study.

I would like to thank my supervisor, Dr Yunqing Xuan for providing his valuable time and help to support me throughout the entire study as his student. I appreciate his patience, guidance and continuous encouragement all the period. His immense knowledge, valuable comments and inspired ideas have helped me to complete my $\mathrm{PhD}$ research and finish this thesis. I have been exceptionally lucky to have a supervisor who cared so much about my work, and who responded to my questions and queries so promptly.

I must express my gratitude to Dr Ryan Bailey from Colorado State University, for his continued support and encouragement. I was continually amazed by his willingness to help me to get knowledge and build the numerical models. Completing this work would have been all the more difficult were it not for the support and friendship provided by the other members of my $\mathrm{PhD}$ office friends and my friends in Iraq. I am indebted to them for their help.

I am very grateful to the Higher Committee for Education Development in Iraq (HCED) for providing me with the scholarship and living funds for pursuing my research. 
I gratefully acknowledge the National Resources Wales, Centre for Ecology and Hydrology (CEH), British Atmospheric Data Centre (BADC), British Geological Survey (BGS), Dr Xiaomeng Song from School of Resources and Earth Science in the China University of Mining Technology, Dr Haider Addab and Ali Al Rammahi from Kufa University in Iraq, for the valuable data that was needed in my research.

I would also like to thank my examination committee members, Professor Dawei Han and Dr Patricia Xavier for serving as my committee members even at hardship. I also want to thank you for letting my defence be an enjoyable moment, and for your brilliant comments and suggestions, thanks to you.

Last but not the least, I would like to thank my brother and my sisters for supporting me spiritually throughout writing this thesis and my life in general. 


\section{List of figures}

Fig. 2-1 Characteristics of simulation and optimisation models ....................12

Fig. 2-2 Types of simulation models .......................................... 16

Fig. 2-3 Classification of hydrological models ..................................20

Fig. 2-4 Multi-level framework of IWRM model integration problems ..............23

Fig. 3-1 River Dee catchment location ..............................................

Fig. 3-2 Average annual precipitation in the Dee River basin ......................35

Fig. 3-3 Land use and soil maps in the Dee River basin .........................36

Fig. 3-4 Significant Water Abstraction Points in the Dee River basin ................41

Fig. 3-5 Schematic representation of conceptual water balance of SWAT model ....43

Fig. 3-6 The main and the upstream catchments of Dee River basin ................47

Fig. 3-7 Graphical representation of the relationship between the uncertainty of parameter and the prediction uncertainty of SUIFI2 algorithm (After Abbaspour et al.,

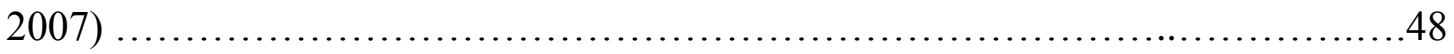

Fig. 3-8 Locations of the main inlets and the river gauge stations in Dee River basin .50

Fig. 3-9 Flow hydrograph at Manley Hall station over both the calibration and the validation periods (Catchment $\mathrm{C}$ ) 52

Fig. 3-10 Flow hydrograph at Druid station over both the calibration and the validation periods (Catchment A)

Fig. 3-11 Flow hydrograph at Bala station over both the calibration and the validation periods (Catchment B) .54

Fig. 3-12 Dotty plots with the objective function of NSE coefficient against each relative change of SWAT parameters at Brynkinalt Weir station .56

Fig. 4-1 Derivation of daily and monthly gridded rainfall estimates of CEH-GEAR (After Keller et al., 2015) 
Fig. 4-2 Methods of precipitation pre-processing for a selected sub-basin in Dee River basin

Fig. 4-3 Comparison of the daily precipitation observed by the gauges in Dee River catchments with the CEH-GEAR data at the same locations for the period of 19952003 .74

Fig. 4-4 Six-month moving average of monthly areal rainfall over the selected subbasins of the Dee river basin for the period of 1995-2003 .75

Fig. 4-5 Spatial distribution of annual rainfall for the three simulations at Dee River basin .76

Fig. 4-6 Per cent of rainfall relative change of sub-basins rainfall for a. GAM against CPEM and b. GPM against CPEM [positive values refer an overestimation rainfall of GAM/GPM over traditional SWAT setup, CPEM] .76

Fig. 4-7 Scatter plots of the simulated and observed flow from the three methods CEPM (a), GAM (b) and GPM (c) respectively at Brynkinalt Weir station for the calibration period .78

Fig. 4-8 Nash Sutcliffe coefficient of cross-validated results of three simulations of the daily SWAT model of Dee river basin for the period of 2001-2003 .80

Fig. 4-9 Determination coefficient of cross-validated results of three simulations of the daily SWAT model of Dee river basin for the period of 2001-2003 .80

Fig. 4-10 The 6-month moving average of monthly river flow simulations at Brynkinalt Weir for the period of 1995-2003 .81

Fig. 4-11 Cumulative monthly flow simulations of Brynkinalt Weir station for the Period of 2001-2003 .82

Fig. 5-1 Annual rainfall in and near Dee River catchment over the period of 19702004

Fig. 5-2 Topographic map of Beijing with the location of rain gauges .92

Fig. 5-3 Areal average rainfall in the metropolitan area of Beijing for each month over the period of 1960-2012 .93 
Fig. 5-4 Visual yearly linear trend for station No. 1 in Dee River basin for different quantiles

Fig. 5-5 Visual yearly quadratic trend for station NO. 1 in Dee River basin for different quantiles .97

Fig. 5-6 Spatial distribution of the linear trend of annual precipitation over Dee River catchment (circular dots represent significant trend points) 102

Fig. 5-7 Yearly linear trends using Quantile Regression for Flooding (tau=0.98) and Drought (tau $=0.02$ ) Conditions over Dee River Basin

Fig. 5-8 Yearly quadratic trends using quantile regression for flooding (tau=0.98) and drought (tau=0.02) Conditions over Dee River Basin 104

Fig. 5-9 Spatial distribution of monthly rainfall of winter months in the Dee catchment (a November, b December, c January and d November - January) for 0.98 quantile [circular dots represent significant trend]

Fig. 5-10 Spatial distribution of the linear trend of annual precipitation of 0.98 quantile over Dee River catchment for R95PTOT and R99PTOT indices (circular dots represent significant trend points) 106

Fig. 5-11 Spatial distribution of the linear trend of annual precipitation of 0.98 quantile over Dee River catchment for R10MM and R20MM indices (circular dots represent significant trend points)

Fig. 5-12 Spatial distribution of the linear trend of annual SPI value for 0.98 and 0.02 quantile over Dee river catchment (circular dots represent significant trend points) 108

Fig. 5-13 Spatial distribution of the significant linear trend of annual precipitation over Beijing metropolitan area .108

Fig. 5-14 Linear quantile regression trends for the areal average precipitation for flooding (tau=0.98), drought $(\mathrm{tau}=0.02)$ and mean $(\mathrm{tau}=0.50)$ conditions over Beijing area 110 
Fig. 5-15 Confidence band of the gradient (mm/year) of the fitted lines using summer (a) and winter (b) seasons in Beijing (The horizontal axes are quantiles and the vertical axes refer to the gradient of the trend lines; the red lines represent the confidence bands of the fits using ordinary linear regression)

Fig. 5-16 Prediction bands of the gradient of observed and simulated river flow trends at Pont-y-Capel station 113

Fig. 5-17 Spatial distribution of the Water Yields trends at 0.98 quantile 114

Fig. 5-18 Spatial distribution of the Water Yields trends at 0.02 quantile 114 Fig. 5-19 Spatial distribution of the Water Yields trends at 0.5 quantile 115

Fig. 5-20 Spatial distribution of the river flow trends at 0.98 quantile for highly managed watershed scenario .116

Fig. 5-21 Spatial distribution of the river flow trends at 0.02 quantile for highly managed watershed scenario

Fig. 5-22 Spatial distribution of the river flow trends at 0.5 quantile for highly managed watershed scenario

Fig. 5-23 Comparison of river flow trends of the sub-basins along the mainstream under Scenario I (highly-regulated) and Scenario II (natural-state) at 0.98 quantile .118

Fig. 5-24 Comparison of river flow trends of the sub-basins along the mainstream under Scenario I (highly-regulated) and Scenario II (natural-state) at 0.02 quantile .119

Fig. 5-25 Comparison of river flow trends of the sub-basins along the mainstream under Scenario I (highly-regulated) and Scenario II (natural-state) at 0.5 quantile 119

Fig. 6-1 Location of the license withdraw wells used in MODFLOW model .132

Fig. 6-2 Schematic representation of conceptual water balance of coupling SWATMODFLOW .134 
Fig. 6-3 MODFLOW grid with the location of groundwater monitoring well in the Dee River basin

Fig. 6-4 Flowchart illustrating the coupled SWAT-MODFLOW model for Dee River watershed 136

Fig. 6-5 Flowchart presenting the model code sequence of the coupled SWATMODFLOW model (After Bailey et al., 2016)

Fig. 6-6 The comparison of simulated river flow from the standalone SWAT model and the coupled SWAT-MODFLOW at Ironbridge for the water year of 1999

Fig. 6-7 The comparison of simulated baseflow from the standalone SWAT model and the coupled SWAT-MODFLOW at Pont-y-Capel for the period of 1995-2000

Fig. 6-8 The comparison of simulated groundwater level from the coupled SWATMODFLOW at the monitoring well for the period of 1995-2000

Fig. 7-1 Location of Alyn River Catchment 147

Fig. 7-2 The scatter plot map for streamflow simulation of SWAT model 156

Fig. 7-3 Location of HRU 74 with Alyn River Basin 157

Fig. 7-4 The scatter plot map for streamflow simulation of the coupled SWATMODFLOW

Fig. 7-5 Average of Elementary Effects against their standard deviation with confidence bounds from bootstrapping .159

Fig. 7-6 Convergence plots of the sensitivity indices of the model parameters for the SWAT and the coupled SWAT-MODFLOW using Elementary Effect Test (EET). The solid lines are the bootstrap means of the sensitivity indices and the dashed lines are the $95 \%$ bootstrap confidence intervals .160 
Fig. 7-7 Scatter plots of parameters for SWAT and coupling SWAT-MODFLOW using GLUE method

Fig. 7-8 Streamflow prediction limits against observations by GLUE method at Ponty-Capel for Alyn River basin for the water year of 1997 163

Fig. 8-1 Elevation map of Iraq with climatic zones 169

Fig. 8-2 Box plot of annual observed precipitation in Iraq 171

Fig. 8-3 Thiessen polygon for observed rainfall in Iraq 172

Fig.8-4 Box plots of average precipitation in Iraq for different temporal bands for period 1941-2005 172

Fig. 8-5 Cullen and Frey graph of areal average monthly precipitation for the observations 179

Fig. 8-6 Goodness of fit of the observed monthly areal average precipitation over Iraq for beta distribution 180

Fig. 8-7 Cumulative of the areal average of observed and modelled precipitation over Iraq 182

Fig. 8-8 Density plots of the areal average of observed and modelled precipitation over Iraq after bias correction

Fig. 8-9 Spatial variation of the annual average rainfall trend using Mann Kendall test over Iraq for a) Observed, b) bcc-csm-1-1 model, c) bcc-csm-1-1-m model, d) CCM4 model, e) MIROC5 model and f) MRI-CGCM3 model 184

Fig. 8-10 Linear trends of the annual rainfall for the observed and simulated time series using the Mann Kendall trend test in zone 2 over Iraq 185

Fig. 8-11 Linear trends of annual rainfall for the observed and simulated time series using the Mann Kendall trend test in zones 3 and 4 over Iraq 185

Fig. 8-12 Linear trends of the areal average annual rainfall for the observed and simulated time series using Mann Kendall trend test over Iraq 186 
Fig.9-1 Management strategies within simulation and allocation models (After Loucks et al., 2005)

Fig. 9-2 Dee River demand sites (After Dee Valley Water and United Utilities, 2017)

Fig. 9-3 Study region of the coupled SWAT-WEAP model (surface water abstraction in $\mathrm{m}^{3} /$ day) 196

Fig. 9-4 Public water supply PWS1 abstractions for scenarios no. 3, 6, 9 and 12 [ 100 $\%$ of maximum licensed abstractions for summer months (June, July and August), 50 $\%$ of maximum licensed abstractions for winter months (December, January and February), and $70 \%$ for the rest with $1 \%$ increase of water use per year] 198

Fig. 9-5 Flowchart of the coupled SWAT-WEAP model framework 199

Fig. 9-6 The average monthly unmet streamflow requirement $\left(\mathrm{m}^{3} / \mathrm{sec}\right.$.) in Chester weir for the four future scenarios for the period of 2008-2040 .200

Fig. 9-7 The average monthly flow requirement coverage (\% of flow requirement) in Chester weir for the four future scenarios for the period of 2008-2040 201

Fig. 9-8 The average monthly unmet demand $\left(\mathrm{m}^{3} / \mathrm{sec}\right.$.) for public water supply PWS for the four future scenarios for the period of 2008-2040 for the maximum withdraw scenario

Fig. 9-9 The average monthly unmet demand $\left(\mathrm{m}^{3} / \mathrm{sec}\right.$.) for public water supply PWS for the four future scenarios for the period of 2008-2040 for the 50\% maximum withdraw scenario 203

Fig. 9-10 The average monthly unmet demand $\left(\mathrm{m}^{3} / \mathrm{sec}\right.$.) for public water supply PWS for the four future scenarios for the period of 2008-2040 for the time series withdraw scenario .204 


\section{List of tables}

Table 2-1: Summary of Allocation models .....................................14

Table 2-2: Summary of Simulation models ....................................17

Table 3-1: Collected data for the SWAT model ..................................... 38

Table 3-2: Characteristics of the major reservoirs in the Dee River basin (Natural Resources Wales, 2015) ..................................................40

Table 3-3: The river gauge stations utilised in the calibration and validation of the hydrological model (Source: Centre for Ecology and Hydrology, 2015) .............50

Table 3-4: The calibrations and validations of the three models A, B and C in the two

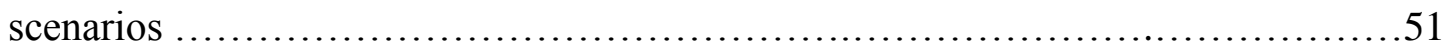

Table 3-5: Calibration (1995-2000) and validation (2001-2003) of the SWAT model .53

Table 3-6: Main SWAT parameters used in the calibration process .55

Table 3-7: Sensitivity of SWAT parameters included in the final calibration and t-Stat and p-values at Brynkinalt Weir station .57

Table 4-1: Statistical comparison of precipitation of observed and CEH-GEAR dataset at rain gauges for a period of 1995-2003 for Dee River .73

Table 4-2: Calibration results of three simulations of the daily SWAT model for Dee river basin for the period of 1995-2000

Table 4-3: The per cent of bias (PBIAS) indices of the SWAT model calibrations at the Brynkinalt Weir station .78

Table 4-4: Percent of bias of three simulations of the daily SWAT model for Brynkinalt Weir station of Dee river basin for the period of 2001-2003 .79

Table 5-1: Quantile regression example applications in environmental and climate change impact studies .94 
Table 5-3: Slope and p-value of trend results of the areal average rainfall over the metropolitan area of Beijing 109

Table 6-1: Collected data for the MODFLOW model 128

Table 6-2: The calibrated river flow of the standalone SWAT model and the coupled SWAT-MODFLOW model for the calibration period of 1995-2000 139

Table 6-3: The calibrated river flow of the standalone SWAT model and the coupled SWAT-MODFLOW model for the validation period of 2001-2003 139

Table 6-4: The simulated baseflow results of the standalone SWAT model and the coupled SWAT-MODFLOW model for the calibration period of 1995-2000 .141

Table 6-5: The simulated baseflow results of the standalone SWAT model and the coupled SWAT-MODFLOW model for the validation period of 2001-2003 141

Table 7-1: SWAT and SWAT-MODFLOW model parameters .155

Table 7-2: Combined parameters for SWAT and coupling SWAT-MODFLOW 155

Table 8-1: Iraqi rain gauges' stations used in this study .170

Table 8-2: The CMIP5 monthly models used in this study 173

Table 8-3: GOFs of CMIP5 monthly areal average rainfall models against observed one 181

Table 9-1: The average monthly unmet streamflow requirement $\left(\mathrm{m}^{3} / \mathrm{sec}\right.$.) in Chester weir for the four future scenarios for the period of 2008-2040 .200

Table 9-2: The average monthly flow requirement coverage (\% of flow requirement) in Chester weir for the four future scenarios for the period of 2008-2040 .201 


\section{Chapter 1: Introduction}

Integrated water resources management (IWRM) is an approach to formulating, utilising and implementing management and planning strategies for ecologically and sustainably developing water resources by considering the temporal and spatial interconnections with human, natural resources and environmental aspects among water users. It is seen as an umbrella concept under which more coordinated, and holistically methods are sought as there is a complex interaction between water resources systems (WRS) and environmental and social sub-systems (Gain et al., 2013). Recently, it has been considered as a global paradigm for water resources management and has been broadly used in dealing with practical water resources management problem (Gain et al., 2012).

According to Agyenim and Gupta (2012), Integrated Water Resources Management IWRM is one of the most proper techniques for the management of water resources for three main reasons:

1) It is a holistically thorough approach that considers all type of water resources;

2) It makes a connection between water resources and catchment's livelihood; and

3) It integrates the aspects of good governance.

The primary objective of sustainable river basin management needs a sound understanding of water resources systems and their types and relationships (e.g. groundwater, surface water, quantity and quality, biotic components, upstream and downstream interactions). The water resources systems should be fully considered and dealt with as part of the broader environment and about socio-economic demands under the effects of the political and cultural situations.

Evidently, water resources management cannot be treated in separation; it is essential to consider the performance of ecosystems simultaneously at different levels and 
different spatial and temporal scales. This often involves management and planning of water system at the various local levels such as field, farm, and village and at the regional levels such as catchments and river basins (Jain and Singh, 2003).

Computer models are abundant when it comes to applications in hydrology and water resources analysis. Hydrological models come as a big group aiming to reveal the processes within a catchment and beyond; hydrodynamic models, on the other hand, are made to account for more detailed physical processes in river channels and floodplains. In the context of IWRM, these models are indispensable tools as to answering the questions related to water quantity, quality, distribution and the impacts of the changes in underlying conditions.

Hydrological models can be used for quantitative studies of IWRM to access surface water resources (runoff) which is often generated by rainfall storms. On the other hand, hydrogeologists make use of groundwater models to simulate the movement of water within water-bearing layers and predict of aquifer system conditions for several purposes such as irrigation development. Sensibly, some output results that are generated from hydrological modelling might be utilised as input for groundwater modelling and vice versa for a more accurate result of available water resources from the natural process.

Operationally, hydrological modelling is a powerful technique through which the hydrological cycle in the real world is presented for prediction of hydrological processes such as surface runoff, evapotranspiration and groundwater recharge. Ordinarily, hydrological models can be divided into two main groups: stochastic and deterministic (Process-based model). Stochastic hydrological models utilise statistical or mathematical techniques (e.g. transfer functions, regression...etc.) to connect input data such as precipitation to the output such as runoff. Whereas, deterministic hydrological models are more complicated as they characterise the physical processes 
in the water resources system (e.g. streamflow, evapotranspiration, subsurface flow...etc.) and they can be a single-event model or continuous simulation model.

What makes the models in IWRM different from those generic, natural-process oriented models, however, is that not only does IWRM need to know 'how much', it also has to deal with policy problems such as 'what can be done with that' in terms of allocation, planning, and the necessary optimisation and decision-making. It is the latter factors that bring in more management-orientated models on top of the traditional, engineering-focused hydrological models and hydrodynamic models.

Model integration or integrated models are a prerequisite for using models to support IWRM. The reason why integrated models are preferred over a single 'super' model is that in any model certain technical compromises always have to be made to competently represent the main processes while purposefully simplifying or even ignoring other less significant ones. Specifically, those already-specialised individual models, if wisely integrated, will outperform a single, supermodel that attempts to address everything.

While integration of water resources management aims to find an optimised approach for addressing and balancing the needs of multiple stakeholders, participants as well as numerous natural components, the use of computer models does not appear to be in an integrated fashion in the first place. However, integration of computer models in IWRM has gradually become significant as the problem of IWRM develops more and more complex requiring more detailed, refined, and dynamic solution to more challenging situations such as climate change impact.

\subsection{Motivations and research questions}

As discussed above, IWRM is a complicated process and needs integration at a different level. It is essential to study hydrological and groundwater flow models and 
all processes affect this model to assess the availability of water resources and investigate the climate change impact on the water resources.

This study aims to use the hydrological model for integrated water resources management under climate change for the highly-regulated river basin. Most studies focused only on one or two aspects of modelling for integrated water resources management where a systematic approach is needed to address issues:

1) How to represent complex human activities in models?

2) How to address input data uncertainties and their implication in IWRM?

3) How to use quantile-based regression to investigate the trend of possible floods and droughts?

4) How to use coupled models to improve baseflow simulation (coupling SWATMODFLOW) and allocation/management (coupling SWAT-WEAP)?

5) Addressing parameters uncertainties of a coupled surface-groundwater modelling (SWAT-MODFLOW)?

6) Understanding the uncertainty in climate projections.

To achieve the study's aims, the research strategy has been designed as follows:

1) A quasi-distributed hydrological model is set up for a highly-regulated river basin, the Dee River watershed in the United Kingdom involving calibration and validation using historical streamflow observation.

2) Use three different methods of precipitation pre-processing to examine their effects on the simulated river flow of the selected study area, fed with both the observed daily rain gauges and the newly gridded rainfall model (Centre for Ecology and Hydrology- Gridded Estimates Areal Rainfall, CEH-GEAR), including cross-calibration and validation.

3) Use quantile regression (linear and nonlinear) to study the trend of the likely floods and droughts based upon the observed rainfall and simulated 
streamflow and water yield of SWAT model for an extended period (more than 30 years).

4) Construct a groundwater flow model and couple it with a calibrated hydrological model to improve the simulation of baseflow.

5) Use the SAFE Toolbox (Sensitivity Analysis for Everybody) to conduct the sensitivity analysis of the of the coupled hydrological-groundwater model.

6) Study the reliability of climate projections by analysing the trends of the observed and simulated precipitation of 18 models of CMIP5 projects with a larger case study area (Iraq) owing to its coarse spatial resolution.

7) Design a range of scenario simulations based on the future climate conditions and feed them into the coupled model to identify the future probably Unmet Demand at four sites with considerable water use for the public water supply.

8) Summarise and conclude the study and with recommendations for future work.

\subsection{Structure of the thesis}

This thesis consists of ten chapters including the introduction in Chapter 1 and conclusion in Chapter 10.

Chapter 2 is a review of the literature of modelling support for integrated water resources management. The classification of models has been discussed and applications are also summarised.

Chapter 3 introduces the study area highlighting the problems of this catchment and building of hydrological modelling using the SWAT model of the complex highlyregulated watershed, the Dee River in the UK. It involves model description, structure and calibration and validation. A summary of the limitations follows the detailed discussion of the model set up. 
Chapter 4 illustrates the use of three techniques of rainfall pre-processing on the simulated streamflow of the SWAT model using rain gauges and gridded precipitation mode (CEH-GEAR model). This setup includes cross-calibration and validation.

Chapter 5 describes the use of linear and non-linear quantile regression to study the trend of regional water resources using the observation (e.g. precipitation) and the simulated results of SWAT model (e.g. streamflow and water yield) for the likely flooding and drought events.

Chapter 6 studies the coupling of the land surface process model (SWAT) with a physically based fully distributed groundwater flow model (MODFLOW) to improve simulation of the baseflow. It includes the steps of model integration and discussion of current application and limitations.

Chapter 7 investigates the parameters uncertainties of SWAT and the coupled SWATMODFLOW using MATLAB toolbox (SAFE).

Chapter 8 examines the reliability of climate projections from CMIP5 to reveal the trend of the historically observed precipitation. Owing to the coarse spatial resolution of the climate model, a larger study area is used, Iraq.

Chapter 9 scrutinises the integration of a simulation model SWAT with an allocation model WEAP as well as the climate model from projections of CMIP5 to develop future scenarios of water uses and evaluate the likely unmet demands.

Chapter 10 concludes the results and identifies the recommendations for future works. 


\section{Chapter 2: A Review on Modelling Support for Water Resources Management}

Computer models play an indispensable role in integrated water resources management (IWRM) providing support to a range of critical components ranging from water resources assessment to management and decision making. This chapter offers a review of computer modelling for integrated water resources management. Firstly, the concept of IWRM is presented in its historical context together with a classification of various related computer models; the two main groups of models: simulation models and the allocation models are then discussed in detail with regards to their structure, conceptualisation, and applications. Finally, this chapter investigates the issues and challenges arising from model integration with a new perspective.

\subsection{Overview}

Integrated Water Resources Management (IWRM) is "an empirical concept built up from on-the-ground experience of practitioners. Although many parts of the concept have been around for several decades - in fact since the first global water conference in Mar del Plata in 1977- it was not until after Agenda 21 and the World Summit on Sustainable Development in 1992 in Rio that the concept was made the object of extensive discussions as to what it means in practice" (Hassing, 2009). It is also an umbrella concept under which more coordinated and holistically methods are pursued as there are complex interactions among water resources systems (WRS) and environmental and social sub-systems (Gain et al., 2013). Recently, IWRM has been considered as a global paradigm for water resources management and has been widely practised to tackle water resources management problem (Gain et al., 2012).

Water resources management has never lacked of attention. The efforts of exploring effective methods for managing limited and yet sometimes excessive water resources can be traced back to as early as the 1930s. Andrews (2006) revealed that the Tennessee Valley Authority in the US was one of the first taking an integrated 
approach to water resources management in 1933. However, the modern form of water resources management is thought evolve from 1970s when water management began to be categorised as an engineering paradigm based on "predict and provide", project-led and sub-sectorial technique to water services (Savenije and Van der Zaag, 2008), with societies capable of various essential engineering measures, such as construction of large-scale irrigation facilities, building dams and reservoirs.

Many researchers in the 1990s, such as Mitchell (1990) addressed those related aspects of water resources management. It was from the early 1990's that water resources management was gradually recognised as a multi-sectoral, multi-regional and multi-dimensional problem-solving process and hence requiring a new concept (Biswas, 2008). A key following-up to this recognition was the proposal of a new paradigm IWRM in river basin management and planning in the early 1990s.

This new approach was one of the first systematic attempts to address many related aspects in WRM practice including environmental protection, stakeholder participation, equity, and efficiency. The concept of IWRM was then summarised in a series of internationally recognised principles, such as the Dublin Principles proposed at the International Conference on Water and the Environment and Rio UNCED Agenda 21. Subsequently, more related standards were used to develop IWRM as a management paradigm by the end of the 1990s when IWRM started to be recognised by a number of key international organisations such as the World Water Partnership (WWP), the UN's Global Water Partnership (GWP) and the World Bank (see Biswas, 2008; Molle, 2009).

Arguably, one of the most preferred definitions of IWRM was given by the Global Water Partnership (GWP, 2012) as "a process which promotes the coordinated development and management of water, land and related resources in order to maximise economic and social welfare in an equitable manner without compromising the sustainability of vital ecosystems and the environment". Some variations were 
also introduced about particular components, processes or mechanisms. For instance, Ballweber (2006) highlighted the role of participants, suggesting that "IWRM is blending or integrating actions and objectives favoured by different players to achieve the best total result within a river basin or watershed". Other researchers associated IWRM with scales and institutions such as River Boards (Maganga et al., 2004), Poverty Index (Mulwafu and Msosa, 2005), Social Learning (Mostert et al., 2008) or even Adaptive Management (Engle et al., 2011). Others such as Savenije and Van der Zaag (2008) used four key dimensions to define IWRM: water resources, water users, temporal and spatial scale.

The practice of IWRM relies heavily on the use of computer-based models from the very beginning, mainly because both assessing and allocating water resources are non-trivial processes. The use of computer models in water resources management naturally followed the two needs in the beginning, i.e., to obtain the knowledge of water resources at given place quantitatively and then to allocate them in an efficient and optimised manner under specific constraints. The former group of computer models is slightly different from those that have been used in studying natural processes in the water cycle, such as hydrological models. IWRM community tends to use more generic names, such as simulation models, to highlight their leading role in IWRM, i.e., simulating natural process to produce resources with different initial and boundary conditions and to form the basis for 'what-if' analysis further.

Computer models in the latter group mathematically resemble an optimisation problem-solving process with the primary objective set as efficient/effective water use under various constraints. Since water use represented by the models in this category is often referred to as allocations of a different part of water resources, these models are also named as allocation models, though, strictly speaking, an allocation is merely a small part of their purposes whereas nowadays more complicated models are focused on decision making in general. The earliest attempt of using computer models in IWRM was probably due to Sheer (1981) where the Potomac Reservoir and River Simulation Model (PRRISM) was used from the late 1960s to early 1970s, to simulate 
the water use from the reservoirs during drought season in the Potomac River Basin for providing public Water supply in Metropolitan area Washington, D.C., USA.

Along with IWRM, models in this field started to grow since the 1960s (Wurbs, 1994). It is fair to say that the modern IWRM would not be able to fully develop without the support of computer-based models. Over the last two decades, the need for detailed, finely granulated models has increased dramatically with many models having been put into use, such as MODFLOW (Niswonger et al., 2011) and PDM (Moore, 2007). While this move, in general, helps practitioners to refine individual models and improve the governance in return, it has fragmented the idea of integration.

Consequently, the more highly refined, specialised models come into use, the less attention is paid to the link between these models. In other words, modelling efforts somewhat has worked in the opposite direction as to the IWRM. Thankfully, over the last decade, researchers have increasingly recognised the problem of using highlyspecialised, fragmenting models in IWRM. Research on model integration in the context of IWRM has appeared in many research agenda, with some promising outcomes as revealed in, e.g., SWAT-MODFLOW (Bailey et al. 2016). Their overall results prove that the model can represent the integrated watershed modelling results that comprise surface hydrological components and groundwater hydrological components with or without well pumping.

Besides, results improve understanding regarding the spatial patterns of groundwater impact on streamflow, which can aid in watershed management schemes of surfacegroundwater conjunctive use. Moreover, in regards to the protection of aquatic species and their sensitivity to streamflow, areas of high groundwater discharge rates and associated baseflow can be identified for protection under changing climate patterns. 


\subsection{Water resources management models}

Computer models, in general, are abundant when it comes to applications in hydrology and water resources analysis. As such, hydrological models come as a big group aiming to reveal the processes within a catchment and beyond; hydrodynamic models, on the other hand, are made to account for more detailed physical processes in river channels and floodplains. In the context of IWRM, those models are indispensable tools as to answering the questions related to water quantity, quality, distribution and the impacts of the changes on underlying conditions.

What makes the models in IWRM different from those generic, natural-process oriented models, however, is that not only does IWRM need to know 'how much', it also has to deal with policy problems such as 'what can be done with that' in terms of allocation, planning, and the necessary optimisation and decision-making. It is the latter factors that bring in more management-orientated models on top of the traditional, engineering-focused hydrological models and hydrodynamic models.

Another key point when using traditionally process-based or physically-based hydromodels in the IWRM context is that they are more often run in a 'simulation' mode under predefined conditions, than being used with real and current situations. This is because those models are used to facilitate the designing and planning of water resources systems or to construct policies that can maximise the favourable impacts and minimise the undesirable ones (Loucks, 2008). From this perspective, models used in IWRM are therefore more often grouped into two categories: the simulation models that answer 'what-if' questions under a set of predefined conditions; and the allocation models that address 'what-should-be-done' questions by seeking optimum operation conditions and policies to meet the increasing water demand (Loucks et al., 2005; Loucks, 2008; Condon and Maxwell, 2013). Figure 2-1 shows the distinction between the two groups of the models used in IWRM. 


\subsubsection{Allocation models}

Allocation models "usually employ some sort of optimisation to maximise the satisfaction of demand while adhering to a variety of system-specific rules and priorities" (Condon and Maxwell, 2013). Allocation models can be part of a decisionmaking process while being linked to different human activities. They are often formulated to represent interactions among various stakeholders to optimise allocations under specific predefined constraints. As such, allocation models are usually based on a simpler form of governing equations and sometimes are datadriven. Accordingly, those models are less computationally expensive compared with the simulation models. It also implies that a Monte-Carlo based operation can be comfortably implemented to account for the stochastic nature of stakeholder interaction and decision-making process.
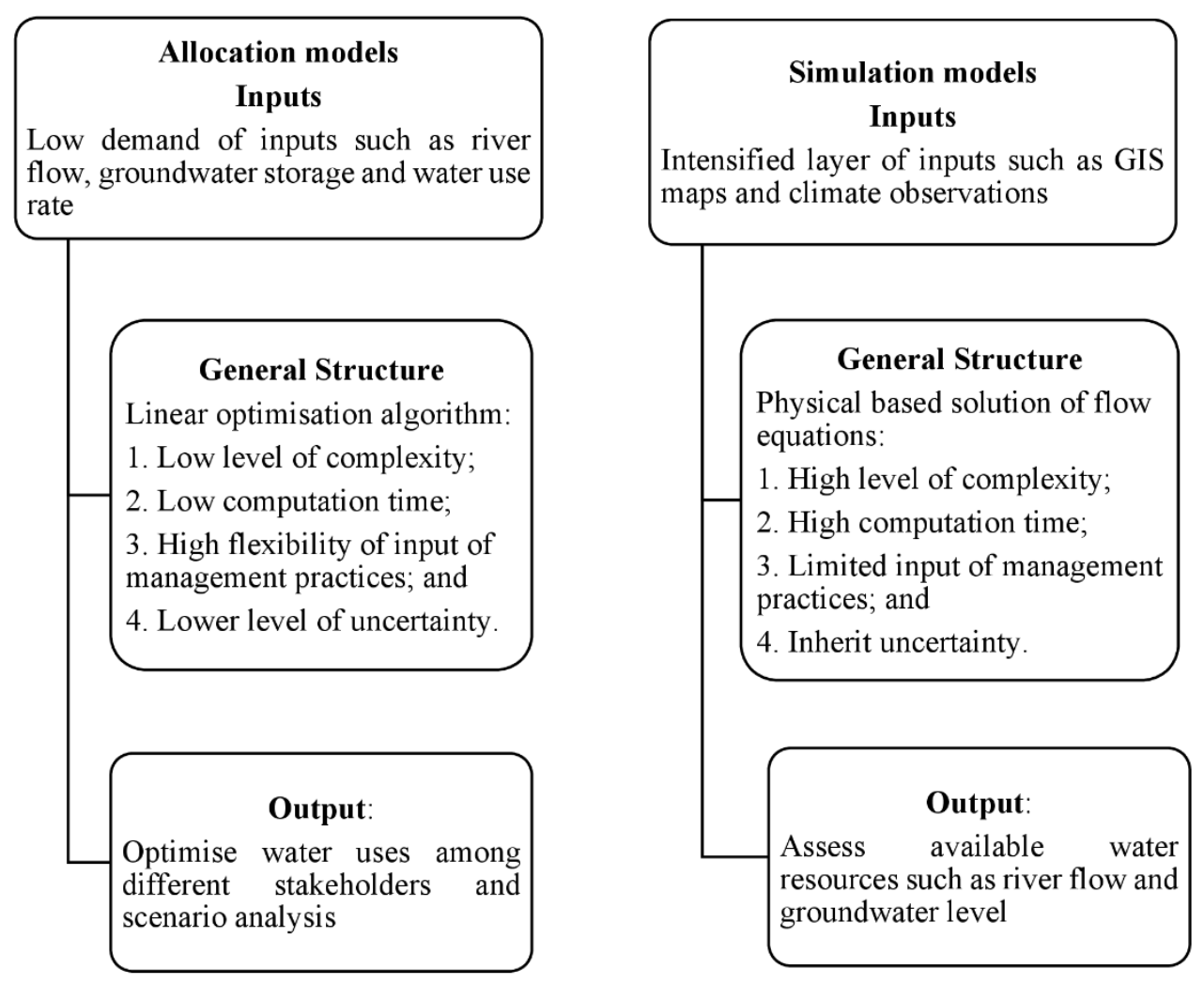

Fig. 2-1 Characteristics of simulation and optimisation models 
While many allocation models are sufficiently competent as far as allocation and decision making are concerned, the main drawback is their inability to account for the feedback from decision's side to the supply side of the resource. In other words, the management practice based on the allocation modelling result may well affect the water supply by altering environments that further change the physical and socioeconomical processes. In fact, the lack of this in allocation models highlights the very need for model integration in IWRM. Several allocation models have been developed and applied, among which the Water Evaluation and Planning System WEAP (Yates et al., 2005), the Resources Allocation Model REALM (Perera et al., 2005) and MODSIM (Ashraf Vaghefi et al., 2017) are three typical models that have been reported in many pieces of literature.

The WEAP (Water Evaluation and Planning) system is "a user-friendly software tool that takes an integrated approach to water resources planning" (Yates et al., 2005). The WEAP model is designed to optimise the allocation of water resources among various users (e.g., municipal, environmental and agricultural) and integrate water quality, water supply and demand. It also offers a link to other simulation models such as groundwater simulation model (MODFLOW), as well as economic model and water quality model (Droogers, 2009). The REALM model aims to simulate the operation of rural and urban, simple and complex water supply systems. Furthermore, the REALM can easily model problems 'what if'. REALM makes use of linear programming to optimise water distribution within a network.

MODSIM (Ashraf Vaghefi et al., 2017) has been applied in complex river basin management that may involve surface-groundwater interaction for conjunctive use of surface and subsurface water resources. MODSIM can also be linked with water quality models for pollution control strategies. Other examples of allocation models include WaterWare (Condon and Maxwell, 2013) and RiverWare (Zagona et al., 2001). 
Table 2-1: Summary of Allocation models

\begin{tabular}{|c|c|}
\hline Main objective & $\begin{array}{l}\text { Optimise the allocation of water resources from various sources } \\
\text { (surface and subsurface water) as a supply to different stockholders } \\
\text { (e.g. Irrigation uses, domestic uses, industrial uses...etc.) }\end{array}$ \\
\hline Formation & $\begin{array}{l}\text { Formed around an objective function of various unknown variables } \\
\text { (decision-variables) to be minimised or maximised with parameter } \\
\text { values assumed to be known and constraints presented as inequalities } \\
\text { and equations. }\end{array}$ \\
\hline Numerical solution & $\begin{array}{l}\text { Normally, data were driven and used to solve a simple form of the } \\
\text { governing equations (i.e. linear optimisation algorithm). }\end{array}$ \\
\hline Interface & $\begin{array}{l}\text { Some models such as WEAP are designed to be able to interact with } \\
\text { other physical models or economic models. }\end{array}$ \\
\hline $\begin{array}{l}\text { Decision Making } \\
\text { Support }\end{array}$ & $\begin{array}{l}\text { Naturally, a part of decision making since it assesses a wide range of } \\
\text { management options and policy scenarios. }\end{array}$ \\
\hline Applications & $\begin{array}{l}\text { Many applications including: development of planning framework } \\
\text { for short-term scenario (land use change) and long-term scenario } \\
\text { (climate change) (Mehta et al., 2013); simulation crop } \\
\text { evapotranspiration (demand) for agricultural land (Joyce et al., 2011); } \\
\text { linking with other models (Ashraf Vaghefi et al., 2015). }\end{array}$ \\
\hline Advantages & $\begin{array}{l}\text { Simply posed with much lower demand for computing resources and } \\
\text { data; thus, suitable for Monte-Carlo based stochastic analysis. It is } \\
\text { also relatively easy for users to prepare input data and assess a sort of } \\
\text { operating policies. }\end{array}$ \\
\hline Disadvantages & $\begin{array}{l}\text { Lack of ability to simulating feedbacks to the physical system and } \\
\text { have limited ability to simulate connections within complex, } \\
\text { heterogeneous, conjunctively management water system. }\end{array}$ \\
\hline
\end{tabular}

Allocation models can be readily characterised by different sub-groups as well. For example, depending on how the allocation process is modelled, the model can be probabilistic, deterministic or a combination of both; subject to the consideration of 
time dependency in the optimisation components, they may be called static or dynamic models; or from perspective of different model structures and algorithms, they are referred to as linear or nonlinear models (Loucks et al., 2005). Table 2-1 summarises the formation and the applications of allocation models.

\subsubsection{Simulation models}

The key input to allocation models is the information of available water resources of the area in question. Model-based evaluation and assessment of water resources have been widely practised long before IWRM started to be implemented. From IWRM perfective, those models fall into another large group - the simulation models, as they are employed to simulate natural processes under given conditions so that water resources can be adequately accounted. Among many other choices, hydrological models that simulate surface water resources and specialised groundwater models are the two main types of models widely used by IWRM practitioners.

Simulation models present certain advantages over allocation tools as some of them can support physically based solutions by solving partial differential forms of the flow equations (Condon and Maxwell, 2013). However, they are often restricted by the expense of computation and are subject to a limited group of operating policies. The use of simulation models allows for integrating physical processes and offers spatially distributed outputs of a wide range of variables. They usually require a wide range of spatially distributed parameters as far as IWRM is concerned.

One must be aware that most models in this group are in fact able to serve different purposes, and water resources management is merely one of them. Besides, models in this groups often have been categorised differently already by other user communities. In the case of hydrological models, they are often referred to as a lumped model versus distributed model, process-based versus physical based, to name just a few. The discussion of these models indeed goes beyond the scope of this chapter, and 
there are large numbers of publications in the relevant fields that can be referred. A distinction, however, does exist between the model being used for its 'normal' purposes and that for serving water management.

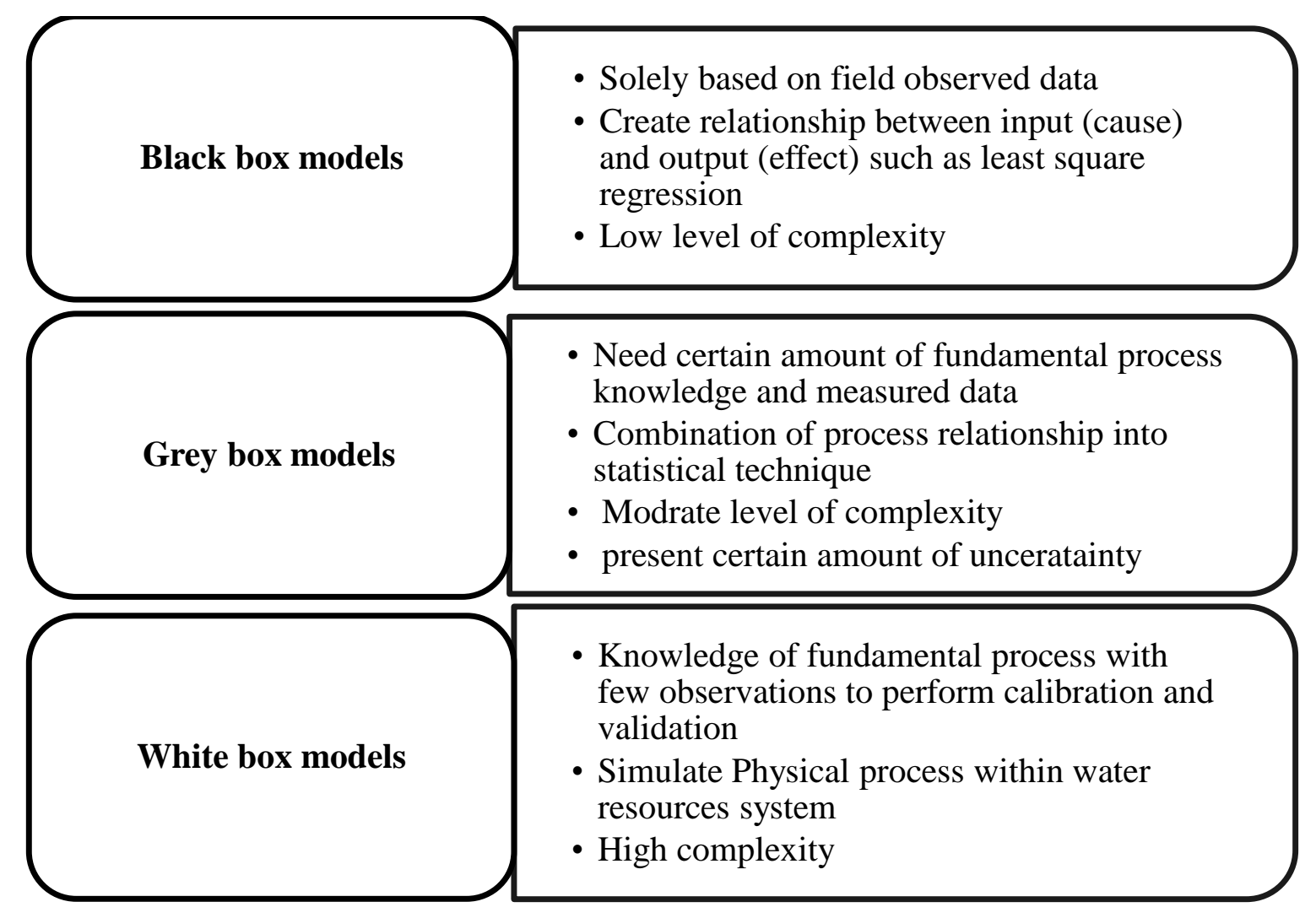

Fig. 2-2 Types of simulation models

Compared with the normal use, water management often needs larger time steps, such as month or year, for planning purposes, and thus requires models to be able to deal with predefined conditions translated from various management options and policies. Therefore, the computing-intensive model with a detailed spatial-temporal output, such as distributed hydrological models may not be necessary for IWRM.

Loucks et al. (2005) further refined the category of simulation models into statistical Black box models), process-oriented (Grey box models) and the combination of both White box models) types, subject to the availability of measured data. Specifically, 
statistical models are driven by a significant amount of measurement conducted in the field so that statistical nature can be revealed. Meanwhile, process-oriented models need the understanding and knowledge of fundamental processes with less availability of data. Figure 2-2 illustrates various types of simulation models. Examples of simulation models used in IWRM are also abundant. Readers can refer to Penn State Integrated Hydrological Modelling System PIHM (Qu and Duffy, 2007), Integrated Water Flow Model IWFM (Miller et al., 2009), Cetemps Hydrological Model CHYM (Verdecchia et al., 2009) and HydroGeoSphere HGS (Brunner and Simmons, 2012). Table 2-2 gives a summary of the simulation models.

Table 2-2: Summary of Simulation models

\begin{tabular}{|c|c|}
\hline Main objective & $\begin{array}{l}\text { Simulating physical processes of the natural water system to assess } \\
\text { the available surface and groundwater resources. }\end{array}$ \\
\hline Numerical solution & $\begin{array}{l}\text { Some of the simulation models need numerical solutions to partial } \\
\text { differential forms of the flow equations and providing support for } \\
\text { physically based solutions. }\end{array}$ \\
\hline $\begin{array}{l}\text { Decision Making } \\
\text { Support }\end{array}$ & $\begin{array}{l}\text { Insufficient ability to dynamically simulate water management } \\
\text { operations decisions }\end{array}$ \\
\hline Applications & $\begin{array}{l}\text { Various examples, including: calculate conditions of surface and } \\
\text { shallow groundwater in the study basin under different management } \\
\text { scenarios (Scherberg et al., 2014); conducted a computation of } \\
\text { groundwater recharge and subdivision water budgets under climate } \\
\text { and topographical gradients (Duffy, 2004); quantified the influence } \\
\text { the shallow tile drain networks on the flow pattern of groundwater } \\
\text { (De Schepper et al., 2015). }\end{array}$ \\
\hline Advantages & Detailed representation of the physical system of water resources. \\
\hline Disadvantages & $\begin{array}{l}\text { Normally restricted by the expense of computation and subject to a } \\
\text { limited group of operating policies and often required a significant } \\
\text { amount of data. }\end{array}$ \\
\hline
\end{tabular}




\subsubsection{Hydrological models}

The rainfall-runoff models or hydrological models range from black box models to be more complicated, differential, distributed models (Tan et al., 2005). Consequently, hydrological models can be classified regarding representing of hydrological processes, space and time scale that is utilised and what techniques of solving model equations (Singh, 1995). The primary features for differentiating the methods are the nature of basic algorithms (i.e. process-based, empirical and conceptual), whether a deterministic or stochastic approach is taken to define input and parameters and whether the spatial representation is distributed or lumped (Melone et al., 2005).

Hydrologic models can be categorised into the following classes based on the existence of random variables, temporal variation and their spatial distribution (Chow et al., 1988):

1) Deterministic models: in this type of models the randomness does not consider, in other words, a specific value of input always results in the same output. Consequently, these models can be utilised for forecasting which is concerned the decision maker for water resources management.

a) Deterministic lumped model: A lumped model takes the spatial average of model parameters of a catchment and does not consider the spatial distribution of the inputs and parameters, and therefore, the basin is treated as a single unit (Cheng, 2011). Thus, the conceptual parameterisation of these is simple and computationally efficient. Lumped models do not provide a reliable and adequate forecast (Melone et al., 2005) which is concerned the decision making for water resources management; for that reason, they are unsuitable for application in this study.

b) Deterministic semi-distributed model: In this kind of model, the basin will be divided into Hydrological Response Units (HRUs) giving the unique value of land use, soil type and slope and simulates the several hydrological processes in each HRU. 
c) Deterministic distributed model: In this type of models, hydrological processes are taking place at each grid and describes the model variables as functions of the space dimensions (Feyen et al., 2000).

2) Stochastic models: The output of these models is at least partially random. Thus, these models create statistical predictions. These models are also classified as space correlated and space independent conditional on whether random variables in space effect on each other.

The broad classification of hydrologic models is shown in Fig. 2-3. The hydrological models can also be classified according to whether the hydrological processes are described as:

1) Empirical models: These models, such as Fuzzy Logic and Artificial Neural Networks, are utilised to create a relationship between rainfall and runoff to predict runoff in different catchments (Chen et al., 2013). These models do not contain physical transformation function to relate input to output;

2) Conceptual models: These model are simplifications of the complex processes of runoff generation in a watershed. The specific components of conceptual models frequently have to be described by empirical functions based on the observation of some processes; and

3) Physical models: These models are distributed based which can explicitly represent the spatial distribution of the mainland surface characteristics such as climatic variables, soil and topographic elevation (Wijesekara et al., 2012).

Catchment models can also be categorised as either continuous or event-based models. On the one hand, event-based models are short-term models utilised for simulating individual storm events, and they form the basis for the design of stormwater infrastructure and as operational models (Melone et al., 2005). Comprehensibly, the main limitation to the use of event-based models is the problem of unknown initial conditions (e.g. initial soil moisture) that cannot be measured and might affect the forecasts in real time (Melone et al., 2005). 


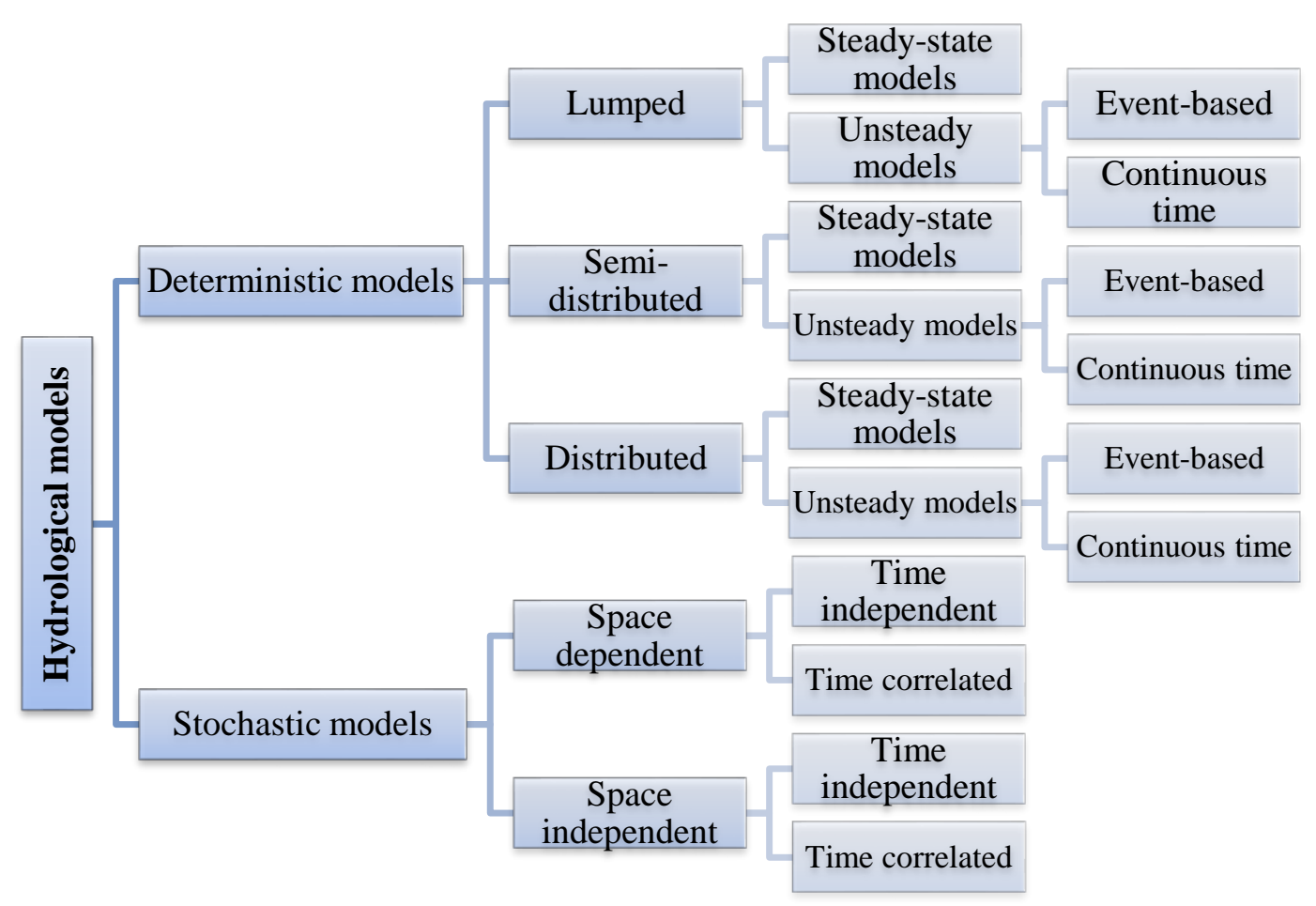

Fig. 2-3 Classification of hydrological models

On the other hand, continuous based models simulate a catchment's overall water balance over an extended period considering all runoff components with providing soil moisture redistribution between storm events (Melone et al., 2005). These models form the basis for water resources planning and management. Accordingly, the use of hydrological models depends mainly (Sahoo et al., 2006) on:

1) Type of model;

2) Modelling skills;

3) Availability of input data;

4) Project requirements and study objectives;

5) Time to process input; and

6) Structure and support to new users.

\subsection{Approaches to model integration}

The classification of models in IWRM reflects the modelling needs in water resources management about various stakeholders, different environment components and heterogeneous water demands. This inevitably assumes allocation models a central 
role while being fed by the simulation models with assessments of water resources. The concept of IWRM in effect requires a closer link between these two types of models. In other words, model integration or integrated models are a prerequisite for using models to support IWRM.

Integrated use of models in water resources management practice has been a continuing effort, from early attempts aiming to partially solve the related problems, such as running the European Hydrological Modelling System SHE (Abbott et al., 1986) to nowadays the so-called seamless integration of several models using specific industrial interface stands such as the Open Modelling Interface OpenMI (Gregersen et al., 2007). The fundamental drive of model integration, if sifted thoroughly, is the need of solving a range of different problems that require multi-instances runs of two or more models working collaboratively. As far as physical problems are concerned, there always should be a single model that can address those entirely related problems, at least in theory.

More recently, there is a trend to use the concept of coupling fine-scale atmospheric models with high-resolution hydrological models (coupled hydro-meteorological models) to decrease uncertainties related to the spatial distribution and timing of heavy precipitation. This is predominantly significant for complex terrain regions (Naabil et al., 2017). One such example is WRF-Hydro model (Gochis et al., 2013) which have been used to successfully predict streamflow and flood forecasting when operating at effective grid resolutions of a few kilometres or even less (e.g. Senatore et al., 2015; Arnault et al., 2015). It also has been applied as an operational tool in assessing water resources such as Naabil et al. (2017). The WRF-Hydro modelling method has been employed in various regions around the world either in a coupled or uncoupled mode (e.g. Fersch, 2014; Kerandi et al., 2018).

Another example is Hydro-JULES programme (the Joint UK Land Environment Simulator) that aims to generate a 3 dimensional model to represent the land- 
atmosphere interactions of water and energy together with terrestrial earth system components such as carbon and nitrogen cycling as well as dynamic vegetation Best et al. (2011) and Clark et al. (2011). The Natural Environment Research Council (NERC) offers the support to its centres for JULES activities including UK Environmental Prediction projects and the Earth System Modelling and other related research for technical development and management with partners.

\subsubsection{Integrated models versus supermodels}

The reason why multiple models are preferred over a single 'super' model is that in any model certain technical compromises always have to be made to competently represent the key processes while purposefully ignore or simplify other less important ones. In other words, those already-specialised individual models, if wisely integrated, will outperform a single, supermodel that attempts to address everything. One of such typical scenarios, for example, is the integration of hydrological model (for the rainfall-runoff process) and the hydrodynamic models (to represent channel and overland surface flow) or even the hydro-meteorological models in many flood risk related modelling efforts, such as Tang et al. (2009).

For IWRM, model integration is naturally a preferred approach. Many practices and case studies, despite having not an explicitly made reference to model integration, have in effect practised in similar fashions. It is because:

1) Allocation models need to have input from simulation models that can assess the available water resources under prescribed conditions; and

2) Decision-based on the model outputs have to be tried out regarding building new conditions on both models to evaluate the impact.

In addition to the traditional approach of model integration between physical models, IWRM has a social dimension that also needs to be integrated, for example, translating the human-made decision into the scenario of integrated models. It is this dimension that distinguishes model integration in IWRM from others that are mainly 
focused on solving physical problems. This also makes it more challenging due to the involvement of translating decisions in the social domain into what can be understood by the physical models.

\subsubsection{Model integration at various levels}

In this section, it is proposed a new, multi-level framework to help pinpoint the model integration problem in IWRM, as shown in Fig. 2-4, a structure that sees the model integration as process-orientated model integration, dataflow-orientated integration, implementation-orientated integration, and finally the presentation-orientated integration.

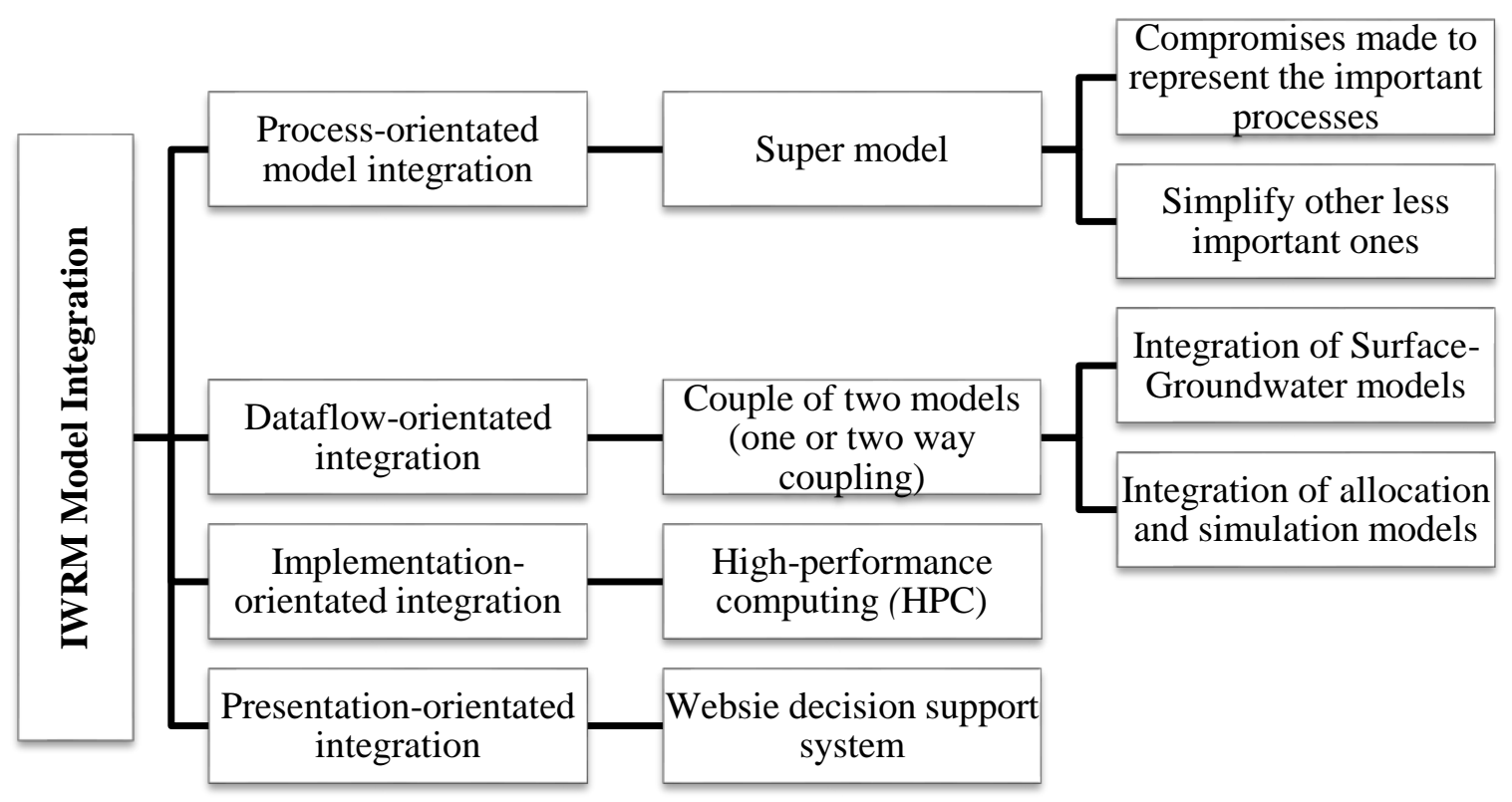

Fig. 2-4 Multi-level framework of IWRM model integration problems

Adjusting model dynamical process representation and sufficiently arranging the data flow across model boundaries is the first step towards model integration in IWRM. They are also like the traditional model integration among physical models. The need of integrating multiple dynamical processes usually arises from simulation models where a more comprehensive physical process needs to be represented. For example, surface water resources and groundwater resources are typically represented by the surface hydrological model and groundwater models and a comprehensive process 
representation of the surface and groundwater interaction would require the integrated use of the two models. In contrast, the dataflow-orientated integration is needed when dealing with data translation, feeding and feedback across different model categories, i.e., simulation models, allocation models and decision support (often included in the allocation models).

When models are linked by certain data flow routes, they are often termed as coupled models. The model coupling can be achieved by linking two simulation models focusing on different processes and/or models belonging to different groups, such as an allocation model driven by a simulation model. Depending on whether the data flow is only one direction or bi-directional, the coupling is often referred to as a 'oneway' or 'two-way' coupling. The use of coupled models has been reported by many researchers, such as: SWAT-MODFLOW (Kim et al., 2008), HEC-RAS-MODFLOW (Rodriguez et al., 2008), SWAT-WEAP (Tegegne et al., 2013), WEAP-Parflow (Condon and Maxwell, 2013) and MODFLOW-WEAP (Hadded et al., 2013).

In IWRM, the importance of interaction between surface and groundwater system is well recognised. However, many models in a coupled mode suffer from inadequate feedback from model boundaries as they have a limited physical representation of the hydrological process. Apart from surface-groundwater interaction, the interaction between allocation and simulation models is far more vital for the decision-making process.

A fully integrated surface-groundwater model would be able to assess the spatially distributed hydrological variables such as river flow, groundwater table and soil moisture content. More significantly, it would also be able to simulate the surface and groundwater interaction in a heterogeneous and complex domain as well as land use change impact on these variables. Construction of coupled surface-groundwater model will need a range of input data such as land use map, soil map, topographic map, aquifer properties data and climate data. 
The accuracy of these kinds of models will be the central part of an IWRM system where water allocation models are driven by the outputs from the integrated surfacegroundwater model to further simulate water management operations for defined operating policies and priorities. This integration framework is beneficial to grasp how the hydrological cycle is affected by management decisions.

A good example of model integration between allocation and simulation models is demonstrated by (Condon and Maxwell, 2013). They developed a water management tool and coupled it with an integrated hydrology model (ParFlow) at the regional scale hydrological system, the Little Washita catchment in the US. The water allocation module illustrated in their study employs a similar linear optimisation method to the Water Evaluation and Planning (WEAP) model. The allocation module they demonstrated is unique because it is coupled with a fully integrated hydrological model and it needed for the better understanding of how management decisions impact the entire hydrological cycle and the coupled water-energy balance. The water management problem reflects the capability of the model to evaluate facing demands and manage unmet water needs in a limited water resources system. Moisturedependent irrigation was utilised for the agricultural demands. Their approach allowed for investigation of managed systems in an integrated fashion not possible with other tools.

\subsubsection{Scaling and uncertainty issues in implementation}

The implementation of model integration, which aims at a higher level of integration, shifts the focus from modelling process of individual models to the more technical side regarding running several models collectively and collaboratively on an integrated Information and Communication Technologies (ICTs) platform. The technical questions at this level include model interface design and implementation, common shared software (operating systems) and hardware (network) platforms support. 
Apart from this seemingly ICT-focused work, there are other critical issues from modeller's viewpoint, among which many integration processes share two challenges:

1) How to match the time scales between two connected models; and

2) How to deal with the uncertainties propagating from one model to another.

Matching different scales among models is one of the necessary steps in model integration. The fundamental reason that individual model chooses to work on different temporal and spatial scales follows the same explanation made above regarding the choice of a single supermodel or running several models in an 'integrated' mode - simply because each model may need to focus on different processes, hence the preferred.

There are two typical scenarios in IWRM where scale matching needs more attention:

1) Integration of spatially distributed or semi-distributed model and spatially lumped model; and

2) The interaction between the fast components (such as surface water) and the slow component (such as groundwater flow).

The so-called distributed model can account for the spatial heterogeneity of processes by discretising large area into detailed, smaller areas that yet can be treated homogeneously, whereas the lumped model regards typically the study area as a single entity by using various parameterisation schemes to represent spatial heterogeneity.

For the mismatch in temporal scales, a good example is that the coupling of a surface hydrological model such as SWAT (Arnold et al., 1998) and groundwater model such as MODFLOW (Niswonger et al., 2011). On the one hand, the SWAT model runs on the sub-daily, daily, monthly and yearly time strep to simulate landscape hydrological processes focusing on the surface phase. On the other hand, the computational time intervals for the MODFLOW is called 'stress periods'. For the steady-state groundwater flow model, stress periods is dimmed. Meanwhile, for the transient-state groundwater flow model, the transient stresses (pumping rates, river stages, etc.) can 
only change at the beginning of each stress period which can be seasons, months, etc. If desired, stress periods can be subdivided into smaller time steps.

Uncertainty in model simulations have been extensively studied in recent years, especially when it comes to hydrological simulations, for example (Shen et al., 2012; Jin et al., 2010; Butts et al., 2004). While the uncertainties associated with a standalone model can well be represented and reduced to some extent, those propagating through model chains or in a coupled model scenario, have yet to be fully explored. In the case of IWRM, there are very few studies having recognised such issue (Hassanzadeh et al., 2016).

Proper accounting for the uncertainty when using multiple, linked models is more paramount than running a single model, since the former scenario may have uncertainty amplified so much that in the end no useful signal can be picked up for decision making. It envisaged that these areas need immediate attention when using multiple models to support IWRM:

1) Implementation of Monte-Carlo based probabilistic simulations and its interpretation by allocation/DSS models;

2) Uncertainty representation and reduction in a coupled model scenario, especially when the coupling is made two-way, with strong feedback within; and

3) Uncertainty-awareness in decision making and optimisation.

To end with, the presentation layer of the model integration works on top of the implementation to present and interact with the relevant stakeholders in an integrated way. Rather than providing static information, such as reports, the integrated presentation should be able to communicate with the users (stakeholders) and conduct what-if analysis based upon the inputs. In addition to serving such purposes, the integrated presentation also needs to efficiently present uncertainty information along with the decision was taken by the user/stakeholder as well as in the final impacts. 


\subsection{Proposed research}

A review of the computer modelling support to water resource management present in the context of IWRM has been carried out. Model classification and their individual development have been discussed focusing on the needs of simulating natural process (simulation models) and the management process (allocation models). It is also argued for even closer integration of those models to support IWRM. Several fundamental issues, such as scaling issues among multiple processes as well as the uncertainty measurement and reduction have been highlighted.

While integration of water resources management aims to find an optimised approach to address, balance the needs of multiple stakeholders, participants as well as multiple natural components, the use of computer models does not appear to be in an integrated fashion in the first place. However, integration of computer modelling in IWRM has become increasingly significant as the problem of IWRM grows more and more complex requiring more detailed, refined, and dynamic solution to more challenging situations such as climate change impact. There are several areas identified in computer modelling where more efforts are worth spending in the context of IWRM:

1) Modelling of management practice and decision-making process. There is an apparent gap in comparison with physical process modelling;

2) Integration of models or modelling systems that belong to two different domains, i.e., the physical process domain and social interaction domain, especially, the understanding of the feedbacks over a range of different temporal scales; and

3) Understanding and managing the uncertainties from the coupled/integrated models. Topics such as decision making under uncertainty should gain more attention.

In this study, The Soil and Water Assessment Tool, SWAT (Arnold et al., 1998) is selected for the following reasons:

1) It is a robust multidisciplinary watershed model; 
2) It is a semi-distributed model that can be used to model spatial heterogeneity of catchment characteristics even without the extensive data requirements of fully distributed models; and

3) The possibility of interfacing this model with other models to develop a range of scenarios to be investigated and analysed.

SWAT model is a physically based, basin scale, quasi-distributed, continuous time hydrological model that operates on a daily time step. This model is designed to predict the impact of management on water, agricultural chemical yields and sediment fluxes in ungauged catchments. SWAT model is computationally efficient and capable of continuous simulation over long time periods which concerns water resources managers.

SWAT model will be utilised to create a hydrological model for the highly-regulated river basin, the Dee River catchment in the United Kingdom to model the interaction of flow regulation, through the physical flow control of reservoirs released in the upstream region, and water abstraction interaction. Two scenarios considered related to the reservoir releases to investigate the impact on the simulated streamflow. The model can be used for climate change impact studies.

The long-term trend of precipitation, as well as the simulated flow and water yields from the SWAT model, will be investigated by using quantile regression. This technique allows for studying the trend for desire quantile rather than average value. In this study, trends of likely flooding (higher quantile) and likely drought (lower quantile) will be considered. The state-of-the-art interfacing of the SWAT model with other environmental models has been achieved to develop the range of scenarios. One of such example is the coupling with MODFLOW, the groundwater flow model, which have been carried out by many researchers such as Kim et al. (2008); Guzman et al. (2015); Bailey et al. (2016) and Bailey et al. (2017). 
SWAT model has a limitation in terms of dealing with groundwater since its groundwater module is lumped and accordingly parameters such as hydraulic conductivity could not be spatially represented (Kim et al., 2008). It also has a limitation to expressing recharge rates and spatial distribution of groundwater levels. On the other hand, in the MODFLOW model, one of the key components is an accurate and reliable calculation of recharge rates within the input data. The groundwater flow of MODFLOW often overlooks the precision of the recharge rates that are needed to be calculated into the model. Therefore, there is significant uncertainty in the simulated results of groundwater flow (Kim et al., 2008).

In this study, the new interface of SWAT-MODFLOW developed by Bailey et al. (2017) will be used in a highly-regulated River basin, the Dee River basin in the United Kingdom to construct daily SWAT-MODFLOW model to improve estimation baseflow of SWAT model in the study area. The SWAT-MODFLOW coupling will be useful for decision makers for water resources planning and management since the coupling of SWAT-MODFLOW reveals the fully distributed water resources model. This model can be used for climate change impact studies.

Moreover, parameters uncertainty of the standalone SWAT and the coupled SWATMODFLOW will be investigated. Apart from the coupling of simulation models (surface and groundwater models), the interaction of simulation and allocation models is far important. In this study, the calibrated SWAT model of the Dee River basin is coupled with climate change data from the CMIP5 project, and then a range of future scenarios are created in allocation model, Water Evaluation and Planning Software WEAP to investigate the likely future unmet water demand for public water supply in the downstream of the study area. 


\section{Chapter 3: Hydrological Modelling of a Highly-regulated River Basin}

As reviewed in Chapter 2, the first group of computer models is typically used in studying natural processes in the water cycle (e.g. hydrological models, hydraulic models and hydrodynamic models). IWRM community tends to use more generic names such as simulation models, to highlight their crucial role in IWRM. The hydrological models are utilised to simulate physical processes of the natural water system to assess the available surface and groundwater resources with different initial and boundary conditions and to form the basis for 'what-if' analysis further.

Hydrological modelling is one of the necessary steps for assessing climate change impact on water resources in river basins. However, many river basins in question already have flow regulations in place which inevitably makes it difficult to model the underlying hydrological process. On the other hand, stakeholders become increasingly keen for more finely-granulated information when it comes to the climate change impacts, such as spatially distributed of water resources under different river flow regulating rules or management practices.

In this chapter, a semi-distributed hydrological model SWAT is utilised as an example of a hydrological model (i.e. simulation model) to model a highly-regulated river basin, the Dee River catchment in the UK which is studied at refined sub-catchment level, with different river flow regulating rules in place as predefined scenarios. The modelling of the Dee River watershed is a challenging process because of its physical flow control through the storage structures from four reservoirs in the upstream side of this river. The Natural Resources Wales in the UK is operating this river to protect the Dee River watershed from flooding in winter and provide water for large surface water abstraction for public water supply PWS in summer.

\subsection{Introduction}

Climate change impact on water-related issues such as floods and droughts have been an active research area thanks to the fact that those physical phenomena and their 
variations are more easily and directly perceived than those that can only be remotely sensed or over a long time, for example, biodiversity (Solomon, 2007). Technically speaking, it is relatively straightforward to make use of many existing hydrological models with climate change projections, especially when rapid advancing computing power makes more climate simulation data available at increasingly high resolutions. It is now possible to simulate regional climate at $1 \mathrm{~km}$ spatial resolution with monthly and daily time step output (Keller et al., 2015).

Accordingly, there have been plenty of studies on climate change impact at river basin level; and unsurprisingly, a key feature shared by them is the use of hydrological models driven by long-term historical forcing fields (such as precipitation) or climate projections for future scenarios, e.g., see (Abbaspour et al., 2009; Ficklin et al., 2013; Jin and Sridhar, 2011; Abdo et al., 2009). More recently, researchers are gradually paying more attention to the uncertainties and biases in such projection driven hydrological simulations (Cannon et al., 2015; Maurer and Pierce, 2014; Miao et al., 2016). For instance, Miao et al. (2016) reported that the bias correction technique they used was beneficial to reduce over $80 \%$ for temperature and $83 \%$ for precipitation of model bias compared with the raw climate model outputs. They also found their method can remove over $40 \%$ and $60 \%$ of the uncertainty in global model temperature and precipitation projections.

Unlike many previously reported studies of climate change impact on general flow trends of largely 'natural' catchments such as Schneider et al. (2013), the literature of those applied to highly-regulated river basins are scarce. There are, however, some attempts, such as Dutta et al. (2015), Yoon et al. (2016), but their focuses were mainly on the modelling procedure per se, whereas, separating anthropogenic impacts from climate change is yet to be addressed. The human activities or physical flow control effects such as hydropower, reservoir, influent return and development of surface and groundwater abstractions become more significant issues in river basins; these activities can cause changes to the hydrology of streams.

The regulated river flow highlights the importance of water in particular river basin, regardless of the management practices are utilised to prevent flooding or provide 
water for various uses. For most rivers, natural river flow data cannot obtain without getting information about human activities. Also, actual evapotranspiration cannot be measured directly for a watershed. For that reason, hydrological models are a powerful tool for the investigating of the hydrological trend and used to separate natural state and management practice within the catchment.

In this chapter, a hydrological model built for a highly-regulated river basin is presented. A medium-sized, highly-regulated (with reservoirs and licensed water withdraws) river basin, the Dee river basin in the UK is studied using the river flow data over more than 30 years (1970-2003). The catchment is modelled using the Soil and Water Assessment Tool (SWAT) focusing on separation and representation of the flow regulation and water abstraction in the sense of restoring the basin to its 'natural' state. The model is then calibrated and validated against the observed data before the two scenarios (with and without regulations) are designed to simulate long-term simulations (1970-2003) of river flow and sub-catchment water yields under the conditions specified by both scenarios. The method can be readily extended to study future climate change impact, and it is also able to incorporate future changes to the regulating rules.

\subsection{Study area}

The Dee River originates from the mountainous region of the Snowdonia National Park in North Wales in the United Kingdom. The main-stream of the river is measured $113 \mathrm{~km}$ long with a catchment area of 2,215 $\mathrm{km}^{2}$ as shown in Fig. 3-1. It flows eastward to the Wales-England border at the City of Chester before discharging into the Irish Sea at the Liverpool Bay. The annual precipitation over the basin shows a clear west-east declining trend with $1,700 \mathrm{~mm}$ in the western part quickly reducing to $685 \mathrm{~mm}$ in the east where flat, lowland dominates as revealed in Fig. 3-2. The temporal distribution of annual precipitation also demonstrates a definite seasonal pattern with wet winters (178-578) mm in DJF (December, January and February) and ordinarily dry summers (165-278 mm) in JJA (June, July and August). Consequently, the Dee river basin experiences both flooding and droughts in different seasons. 
Records show that the storms and subsequent flooding in the winter of 2013/2014 had a significant impact on some communities, businesses, infrastructure and the environment. There could be more extremes in the weather with a changing climate leading to more frequent and more severe flooding (Natural Resources Wales and Environmental Agency, 2014). Additionally, the droughts in 1995 and 1996 are the most notable recorded drought event of the Dee River basin (Mayall, 2000).

According to Natural Resources Wales (2015), the Dee River basin is one of its examples of advanced river basin management that is mainly achieved by:

1) Regulating the upstream river flow through controlling the release of water from the four main reservoirs: Celyn, Brinig, Bala and Alwen;

2) Sustaining substantial surface water withdraws by adjusting reservoir release in summer in the middle and downstream for industrial and public water supply; and

3) Reducing flood risks in the middle and downstream during wet seasons.

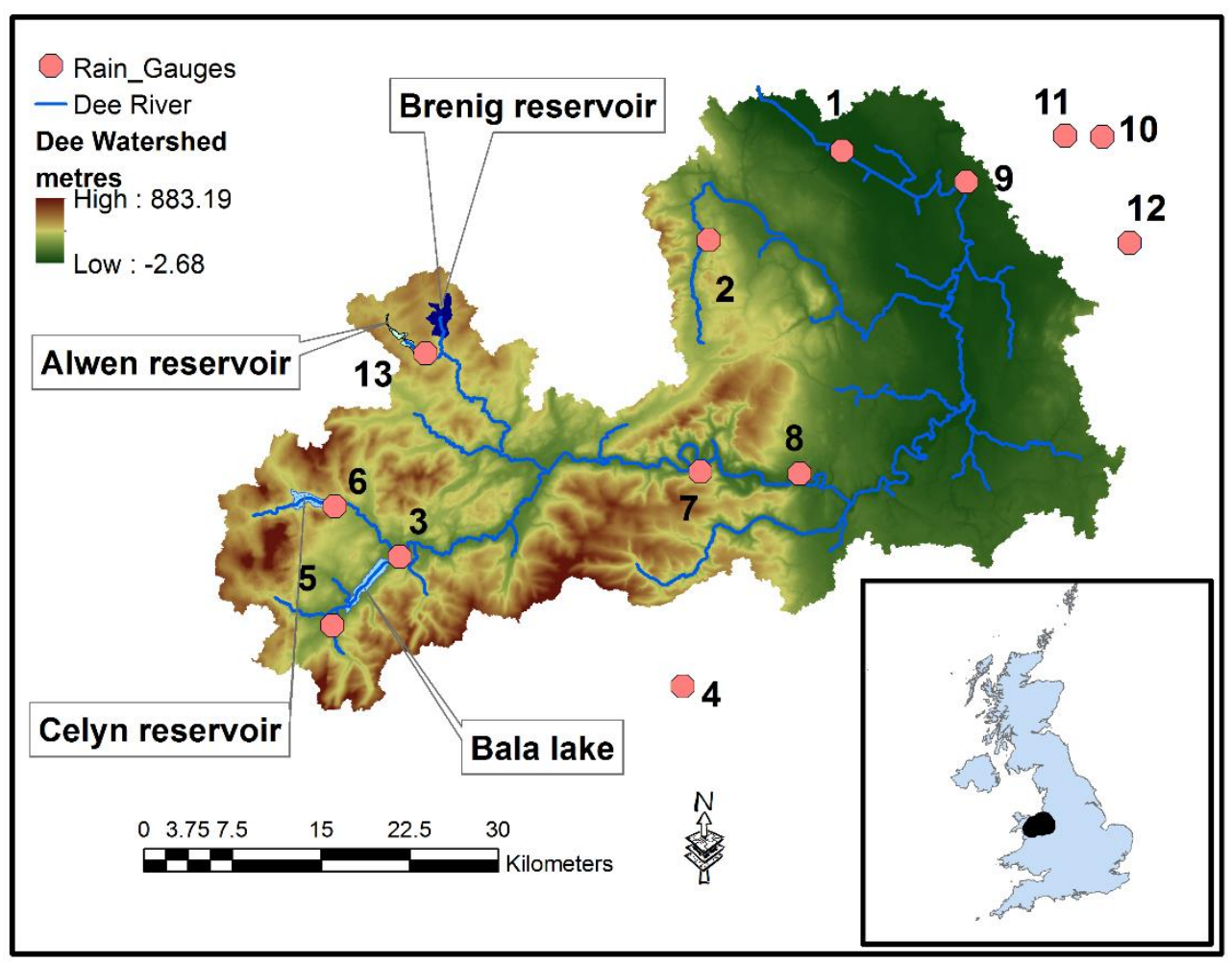

Fig. 3-1 River Dee catchment location 
The management of the river basin has been aimed to maintain industrial and domestic water supply to over three million people for regions of Wirral, Cheshire, Shropshire and north-east Wales (Natural Resources Wales, 2009). In the meantime, flood risks in the downstream urban areas such as the city of Chester have been effectively reduced (Natural Resources Wales and Environmental Agency, 2014).

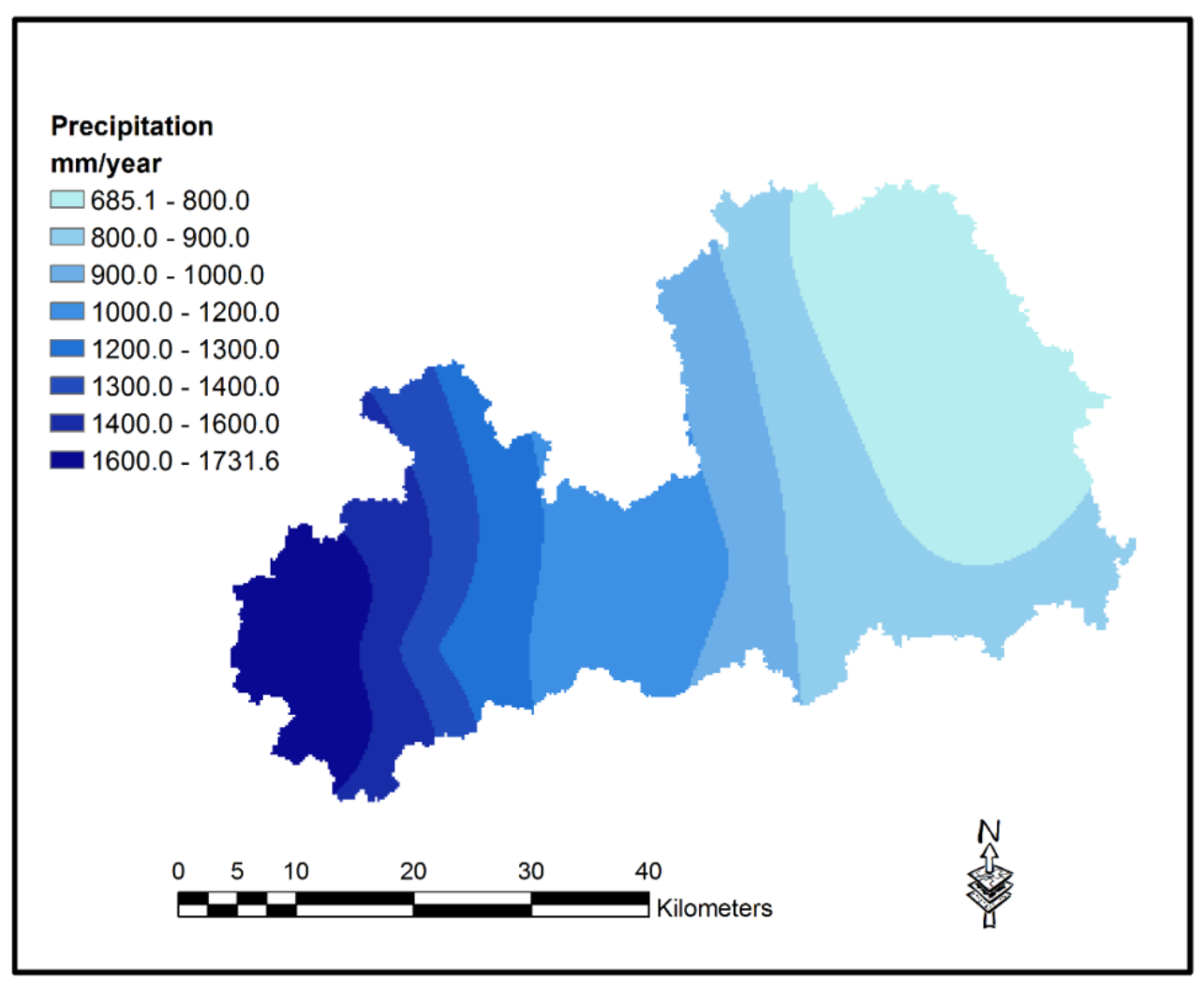

Fig. 3-2 Average annual precipitation in the Dee River basin

The Dee River is managed by Natural Resources Wales (NRW) through a regulatory scheme. The PWS surface water abstractions from the River Dee are governed by the River Dee General Directions which set out rules for the licensed water withdraws during drought conditions and are approved by the statutory Dee Consultative Committee (DEFRA, 2014).

If storage in the regulating reservoirs falls to the drought action trigger level, a meeting of the Committee will take place to discuss the introduction of drought alleviation measures as enshrined in the Dee General Directions. To a large extent, 
water flow in the Dee and certain of its tributaries is regulated under a set of rules called the Dee General Directions, a requirement of the Dee and Clwyd River Authority Act 1973 (DEFRA, 2014). They comprise:

1) "Normal General Directions" which are employed during times of "normal" flows; and

2) "Drought General Directions" which are specified to define the principles and detail under which the prescribed flows and abstractions must be reduced in a drought, more severe than the design drought.

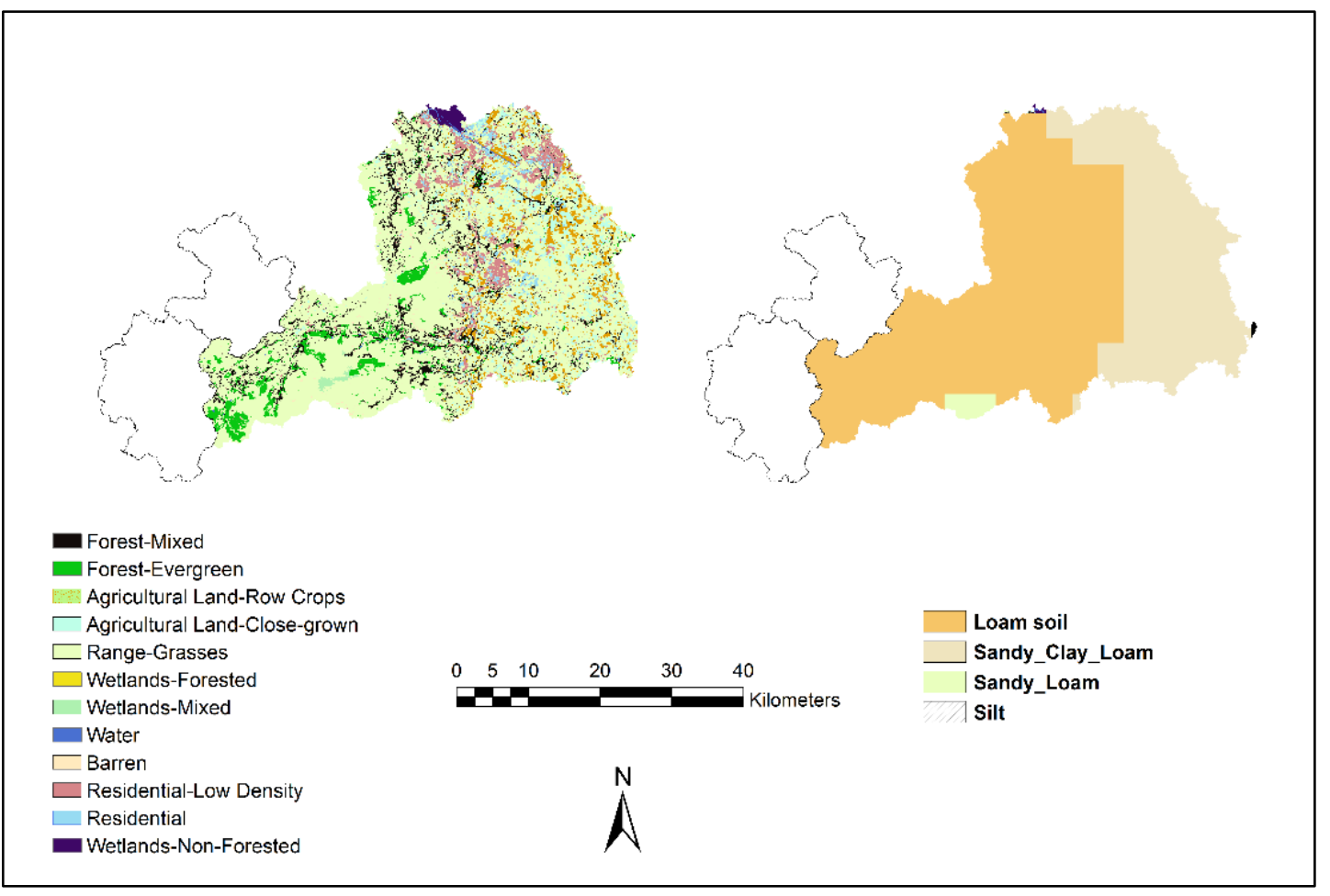

Fig. 3-3 Land use and soil maps in the Dee River basin

These rules are introduced when the total storage of the Llyn Celyn and the Llyn Brenig reservoirs fall below the seasonal "System Conservation Rule Curve" (SCRC). The flows in the Dee are controlled by the River Dee regulation scheme which comprises a system of flow balancing along the River Dee. There are four main lakes/reservoirs in the upstream of the Dee River Basin: The Bala Lake, the Celyn Reservoir, the Brenig Reservoir and the Alwen Reservoir which are used primarily as a water supply reservoir for Welsh Water (DEFRA, 2014). 
The River Dee Regulation Scheme utilises the storage in the Celyn reservoir, the Brenig reservoir, and the Bala Lake to ensure that up to $733 \mathrm{Ml} /$ day can be abstracted in the lower reaches for public water supply. In terms of a hierarchy of the use of the water from the reservoirs, the release of water is as follows (DEFRA, 2014):

1) Bala lake;

2) Celyn Reservoir; and

3) Brenig Reservoir.

The Bala lake is controlled to hold a" buffer" of 0 to $20 \mathrm{~m}^{3} / \mathrm{s}$. of water. Once it holds more than this, it will "spill" (DEFRA, 2014). Figure 3-3 shows land use and soil map of the study area. The mainland use categories in the study area are range-grasses (67 $\%)$, forest (12\%), agriculture (13\%), residential region (7\%) and wetland (1\%). The most dominant soils are loam and sand as presented in Fig. 3-3.

\subsection{Data collection}

There are four categories of data collected to model the catchment, namely:

1) The static dataset, such as DEM, land use and soil type data that are presumed to be static over the study period;

2) The historical observations of precipitation and river flow data, as well as temperature data;

3) CEH-GEAR daily data; and

4) The operational data of flow regulation and water abstraction data that represent management practice.

Most data used are available in the public domain except those requested from the water management authority subject to an academic license. The summary of the data is illustrated in Table 3-1. 
Table 3-1: Collected data for the SWAT model

\begin{tabular}{|c|c|c|}
\hline Data & Resolution & Source \\
\hline $\begin{array}{l}\text { Digital Elevation } \\
\text { Model DEM }\end{array}$ & $25 \mathrm{~m}$ & $\begin{array}{l}\text { ASTER Global Digital Elevation Model } \\
\text { Version 2. NASA. }\end{array}$ \\
\hline Land Use Map & $25 \mathrm{~m}$ & Centre for Ecology and Hydrology \\
\hline Soil Map & $3.5 \mathrm{~km}$ & $\begin{array}{l}\text { Digital Soil Map of the World and Derived } \\
\text { Soil Properties. FAO. }\end{array}$ \\
\hline River Network & $\begin{array}{l}1: 15,000 \text { to } \\
1: 30,000\end{array}$ & $\begin{array}{l}\text { OS Open Rivers Ordnance Survey (GB), } \\
\text { EDINA maps }\end{array}$ \\
\hline River Flow Data & Daily (1970-2003) & National River Flow Archive, CEH \\
\hline Precipitation & Daily (1970-2003) & $\begin{array}{l}\text { Met Office - MIDAS Land Surface Stations } \\
\text { data. British Atmospheric Data Centre. }\end{array}$ \\
\hline $\begin{array}{l}\text { CEH-GEAR } \\
\text { precipitation }\end{array}$ & $1 \mathrm{~km}$ & The Centre for Ecology and Hydrology \\
\hline Air Temperature & Daily (1970-2003) & $\begin{array}{l}\text { Met Office - MIDAS Land Surface Stations } \\
\text { data }\end{array}$ \\
\hline Reservoir Release & Daily (1970-2003) & Natural Resources Wales. \\
\hline $\begin{array}{l}\text { Surface and } \\
\text { Groundwater } \\
\text { withdraws }\end{array}$ & -- & Natural Resources Wales (2015). \\
\hline
\end{tabular}

\subsection{Methodology}

As mentioned before, hydrological modelling of the river basin is widely used to reveal regional water resources and their variability. In this study, the Soil Water Assessment Tool SWAT (Arnold et al., 1998) is chosen for this purpose. This model is wide-reaching used to successfully investigate the impact of catchment management on:

1) Water availability (e.g. Wang et al., 2016);

2) Nutrients fluxes (e.g. Malagó et al., 2017);

3) Sediments transports (e.g. Vigiak et al., 2017);

4) Climate change (e.g. Kankam-Yeboah et al., 2013);

5) Agricultural yields (e.g. Bannwarth et al., 2014); and

6) Land use impact studies (e.g. Babar and Ramesh, 2015). 
However, it is the treatment of river flow regulation and the representation of water use and abstraction that makes this study distinctive from others. Two scenarios concerning the choices of river flow regulation are constructed to assess the impact of the management practice. Then in the next chapter, different methods of precipitation pre-processing on the hydrological modelling performance and model calibration and validation.

\subsubsection{Flow regulation- water abstraction interaction}

The observed river flow data are in fact the result of the natural flow process regulated by the management rules which in turn is based upon the estimate of probable water use in the central water withdraw area. In other words, the natural rainfall-runoff relationship cannot be revealed only by the data per se. For the Dee catchment, releases from the four upstream reservoirs contribute substantially to the river flow; for instance, the catchment area above Chester Weir is $1,816 \mathrm{~km}^{2}$ with an average natural runoff of $36.8 \mathrm{~m}^{3} / \mathrm{sec}(639 \mathrm{~mm} /$ year) and sixteen percent of the area, and $33 \%$ of this runoff are controlled by the regulating reservoirs (DEFRA, 2014).

Water released from the Celyn Reservoir goes into the River Tryweryn and then flows into the River Dee. Most of the water passes through a small hydro-electricity plant to generate electricity. There are three hydropower licences. The catchment of the Brenig reservoir is significantly "over-reservoired" (DEFRA, 2014). It means that the reservoir cannot usually fill from its own watershed within one annual hydrological cycle.

When the reservoir level is drawn down, it can take several years for it to completely re-fill again. The Brenig Reservoir is therefore only used during drought conditions when the capacity of the Celyn and the Bala Reservoirs are no longer predicted to be capable of maintaining the flow in the River Dee. The Bala Lake is a natural lake but now forms part of the River Dee regulation system and the level of its outflow is automatically controlled. The Alwen Reservoir is used as a direct source for public water supply. It is not part of the Dee regulation system. Table 3-2 provides the main 
reservoir characteristics of the main reservoirs in the upper reaches of the Dee catchment.

Table 3-2: Characteristics of the major reservoirs in the Dee River basin (Natural Resources Wales, 2015)

\begin{tabular}{cccccc}
\hline $\begin{array}{c}\text { Reservoir } \\
\text { Name }\end{array}$ & $\begin{array}{c}\text { The } \\
\text { height of } \\
\text { Dam }(\mathbf{m})\end{array}$ & $\begin{array}{c}\text { Total Storage } \\
\text { Volume }\left(\mathbf{1 0}^{\mathbf{6}} \mathbf{~ m 3}\right)\end{array}$ & $\begin{array}{c}\text { Surface Area } \\
\text { (hectares) }\end{array}$ & $\begin{array}{c}\text { Average } \\
\text { Runoff } \\
\left(\mathbf{m}^{\mathbf{3}} / \mathbf{s}\right)\end{array}$ & $\begin{array}{c}\text { Average } \\
\text { Runoff } \\
(\mathbf{m m} / \mathbf{y e a r})\end{array}$ \\
\hline Celyn & 58 & 81 & 325 & 3.10 & 1590 \\
Brenig & 50 & 60 & 370 & 0.62 & 884 \\
Alwen & 30 & 15 & 150 & 0.73 & 899 \\
Bala Lake & N/A & 18 & 400 & 11.50 & 1380
\end{tabular}

There are circa 30 Public Water Supply (PWS) licences with substantial abstractions in the area. In 2009, the PWS abstracted a total of 197,042 million litres which accounted for approximately $93 \%$ of all the water abstracted in the Dee Catchment Abstraction Management Strategy (CAM) area. Of the water abstracted by PWS companies in 2009 only around 1\% was taken from groundwater sources (DEFRA, 2014). The locations of these PWS licenses are shown in Fig. 3-4. Although the metric capacity data of the water abstraction points along with their positions are known, the real-time water abstraction data are not available. According to the management practice where the planned water abstraction determines reservoir releases on the day, the following procedure to inversely represent the day water abstraction at each site of PWS can be used.

To conserve water supplies and ensure efficiency of operation, the PWS companies provide a weekly abstraction forecast to Natural Resources Wales to assist in calculating the required releases from the reservoirs (DEFRA, 2014). An inversion of this procedure is used to estimate the daily water withdrawal amount at the water abstraction points, as this amount is not available from the data collected. 


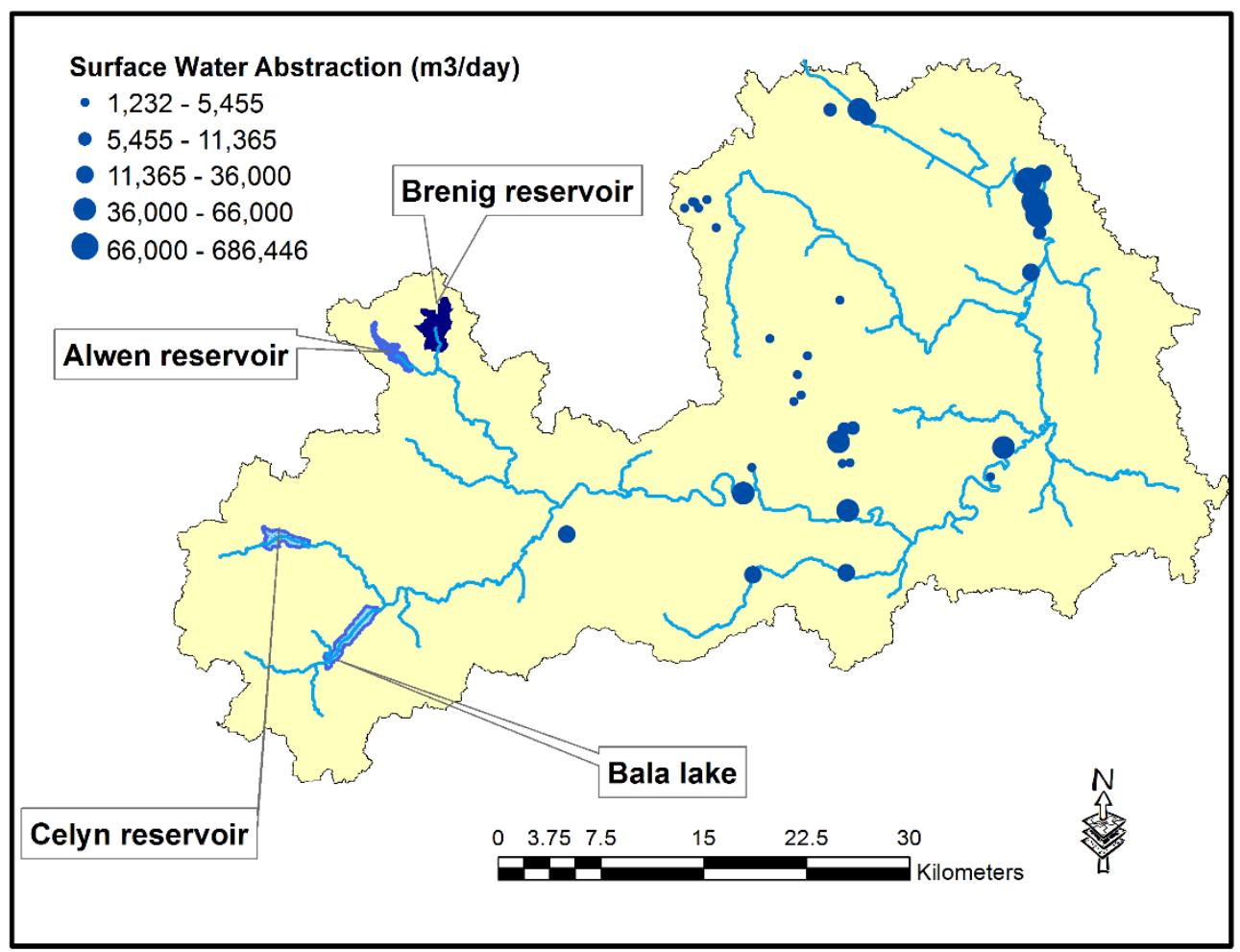

Fig. 3-4 Significant Water Abstraction Points in the Dee River basin

The $i^{\text {th }}$ day water withdrawal at an abstraction point $k$, denoted as $W_{i, k}$ can be estimated as:

$$
W_{i, k}=\mathrm{CNR}_{i} \times M D L A_{k}
$$

where $\mathrm{CNR}_{i}$ is the coefficient of the normalised reservoir release on the $i$ th day, and MDLA $_{k}$ refers to the Maximum Daily Licensed Abstraction at point $k$. The normalised reservoir release from the three reservoirs (Celyn, Brinig and Alwen) $\mathrm{NR}_{i, j}, j=1,2,3$ is calculated by nomarlising the $i^{\text {th }}$ day release over the span between the minimum and maximum releases over the entire period of simulation, i.e., 19702003:

$$
N R_{i, j}=\frac{R_{i, j}-M I N R_{j}}{M A X R_{j}-M I N R_{j}}, j=1,2,3
$$

The coefficient is then calculated by summing up the three normalised releases: 
$\mathrm{CNR}_{i}=\sum_{j=1}^{3} N R_{i, j}$. It is possible that $\mathrm{CNR}_{i}$ may exceed 1 in which case 1 is used as the upper limit. The main reason of aggregating reservoir releases is due to a hierarchy of the use of the water from the reservoirs (larger amount from Celyn reservoir and smaller from Alwen reservoir). Following the procedure above, a 'restored' daily flow time series is produced and ready to be used for the subsequent modelling purposes.

Understandably, this method, although helps to represent the interaction between flow regulation and water abstraction, it also brings in uncertainties. It can be improved by further modelling the probabilistic nature of water abstraction or by conditioning them on other variables such as temperature.

\subsubsection{Modelling streamflow using SWAT}

The Soil and Water Assessment Tool, SWAT (Arnold et al., 1998) is a public domain hydrological model which has been tested in many applications in different parts of the world. It is a physically-based continuous river basin scale model and is designed to simulate the rainfall-runoff process under various spatial and temporal scales. Moreover, this model is a spatially quasi-distributed using hydrological response units (HRUs) to describe the spatial distribution of soil characteristics, land use, topography within a catchment. The simulation of the hydrology of a watershed can be divided into two main divisions (Neitsch et al., 2011):

1) The land phase of the hydrological cycle which controls the amount of water, sediment, nutrient and pesticide loadings to the main channel in each subbasin; and

2) The routing phase which can be defined as the movement of variables mentioned above through the stream networks of the watershed to the outlet.

The calculations in SWAT are performed for each HRU and then scaled up to the subbasin outlet by the per cent of an area of the HRU within the sub-basin. This approach results in the HRUs lacking spatial relations typically seen in a fully distributed model but yield a computationally efficient calculation scheme allowing for rapid watershed 
simulation over long time periods (Bailey et al., 2016). The details of the model structure, applications as well as model set-up are widely available, e.g., in Abbaspour et al. (2015).

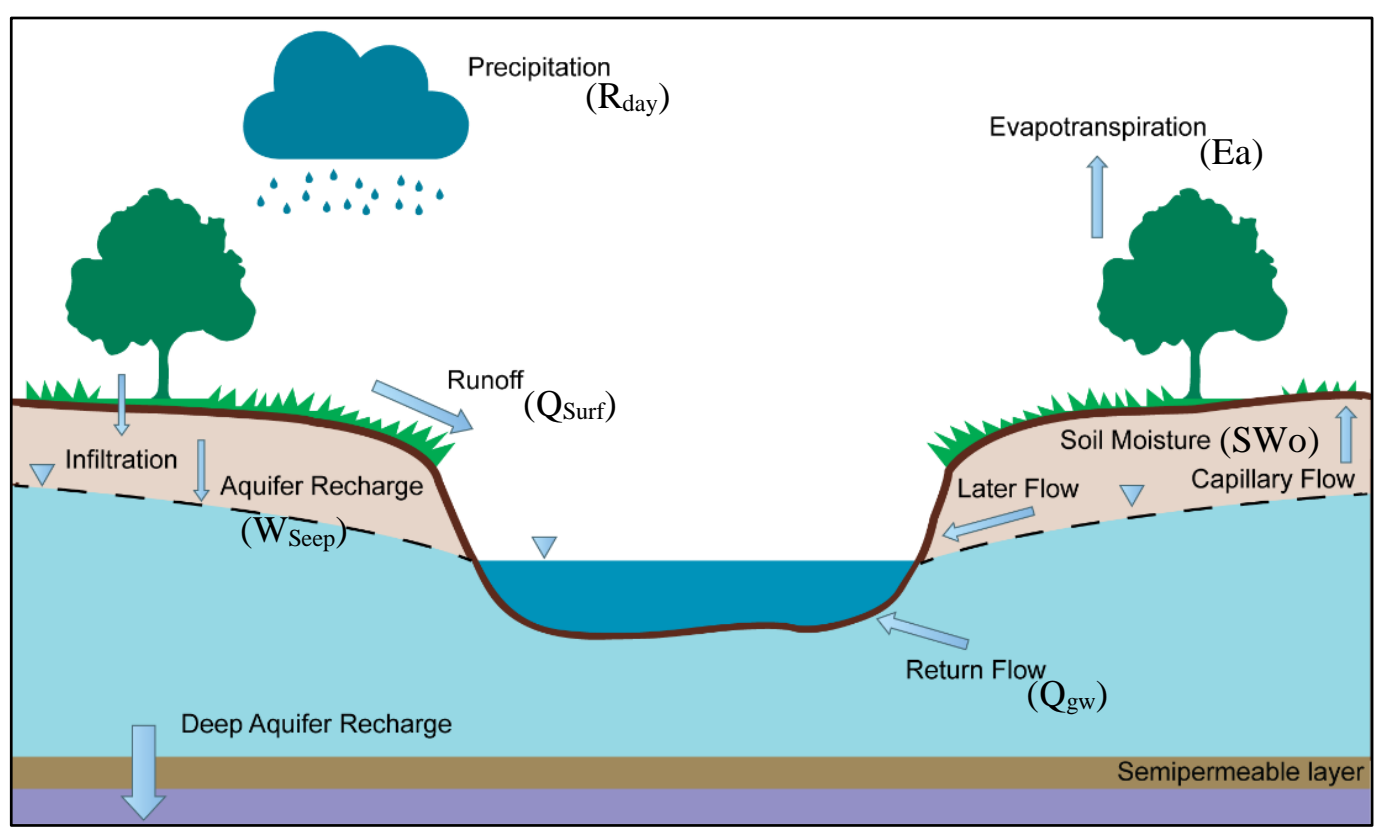

Fig. 3-5 Schematic representation of conceptual water balance of SWAT model

The division of the watershed enables the model to reflect differences in evapotranspiration for different soil and crops. Runoff is calculated separately for each HRU and routed to obtain the total runoff for the watershed. This increases the accuracy and provides a better physical representation of the water balance (Neitsch et al., 2011). Figure 3-5 shows a schematic representation of a conceptual water balance of the SWAT model. It is of particular interests to mention that in the land phase, SWAT simulates the hydrological cycle based on the water balance equation (Neitsch et al., 2011) as follows:

$$
S W_{t}=S W_{o}+\sum_{i=1}^{t}\left(R_{\text {day }}-Q_{\text {surf }}-E_{a}-W_{\text {seep }}-Q_{g w}\right)_{i}
$$

where:

$S W_{t}$ : Final soil water content $(\mathrm{mm})$, 
$S W_{o}$ : Initial soil water content on day $i(\mathrm{~mm})$,

$t$ : time in (days),

$R_{\text {day }}$ : the amount of precipitation on day $i(\mathrm{~mm})$,

$Q_{\text {surf: }}$ the amount of surface runoff on day $i(\mathrm{~mm})$,

$E_{a}$ : the amount of evapotranspiration on day $i(\mathrm{~mm})$,

$W_{\text {seep }}$ : the amount of water entering the vadose zone from soil profile on day $i(\mathrm{~mm})$,

$Q_{g w}$ : the amount of return flow on day $i(\mathrm{~mm})$.

The surface runoff $Q_{\text {surf }}$ is predicted for daily rainfall using SCS curve number equation (USDA-SCS, 1972) as:

$$
\begin{gathered}
Q_{\text {surf }}=\frac{(R-0.2 s)^{2}}{R+0.8 s}, R>0.2 s \\
Q_{\text {surf }}=0.0, R \leq 0.2 \mathrm{~s}
\end{gathered}
$$

where $s$ is a retention parameter. The retention parameter is varied (Arnold et al., 1998):

1) Among watershed, because land use, soil, slope and management all vary; and

2) With time owing to changes in soil moisture content.

The retention parameter $s$ is related to curve number $C N$ in the following equation:

$$
s=254\left(\frac{100}{C N}-1\right)
$$

The constant, 254, gives $s$ in mm. Fluctuation in water content makes $s$ parameter to be changed according to the following equation:

$$
s=s_{1}\left(1-\frac{F F C}{F F C+\exp \left[w_{1}-w_{2}(F F C)\right]}\right)
$$


where $s_{1}$ is the value of $s$ associated with $C N_{l}, F F C$ is the fraction of field capacity and $w_{1}$ and $w_{2}$ are shape parameters. FFC can be calculated as follow:

$$
F F C=\frac{S W-W P}{F C-W P}
$$

where $S W$ : is the soil water content in the root zone $(\mathrm{mm}) . W P$ is the welting point water content $(\mathrm{mm}),(1,500 \mathrm{kPa}$ for many soils $), F C$ is the field capacity water content (mm) (33 kPa for many soils).The values of $w_{1}$ and $w_{2}$ are determined from a simultaneous solution of Equation (3-7) according to the assumption of $s=s_{1}$ when $F F C=0.6$ and $s=s_{3}$ when $(S W-F C) /\left(W_{\text {seep }}, o-F C\right)=0.5$ (Arnold et al., 1998).

SWAT provides two methods to calculate surface runoff: SCS curve number (USDASCS, 1972) and Green Ampt infiltration (Green and Ampt, 1911). The choice of the rainfall-runoff model is often a trade-off between model complexity (simple vs complex) and the availability of required input data. While the curve number has been considered to produce satisfactory results, it is a theoretically simple model based on empirical relationships between daily rainfall, type of soil and land use category without considering rainfall intensity or duration of storm events. On the contrary, the Green Ampt is a physically-based method and can model storm events owing to the condition of sub-daily precipitation, a model input that can be challenging to get. In this study, the technique of the SCS curve number is utilised to estimate the surface runoff since the objective of the study is to create a daily hydrological model.

The peak in this model is calculated using the modified rational method. Additionally, SWAT model offers three techniques to estimate potential evapotranspiration (PET): Penman-Monteith method (Monteith, 1965), Priestley-Taylor method (Priestley and Taylor, 1972) and Hargreaves method (Hargreaves et al., 1985). In this study, the Hargreaves method is employed to estimate potential evapotranspiration since this technique required only temperature data and this variable is available for long records. The actual evapotranspiration (ET) is computed based on Ritchie (Ritchie, 1972). 
In this study, the standard approach of the SWAT model setup is mostly followed. However, to measure the impact of management practice, the entire river basin is modelled with two different configurations: Scenario I and II, which differ from their objectives as well as the areas they represent. Considering data availability, the entire catchment of Dee is separated into three parts (Fig. 3-6). The two upper-stream subcatchments cover the three water supply reservoirs whose releases are measured at the two inlets to the central part - sub-catchment $\mathrm{C}$ which constitutes the main study area. Correspondingly, there are three SWAT models set up for sub-catchments A, B and C, namely models A, B and C.

Scenario I focuses on a so-called 'real-life' situation, i.e., to model the catchment as it is. In this setting, the observed flow at the outlets of both sub-catchments A and B (i.e., the two inlets to sub-catchment $\mathrm{C}$ ) are used to build Model $\mathrm{C}$ alongside historical datasets. The daily water abstractions at the PWS sites are estimated using the method presented above. Neither of the sub-catchments in the upper stream are modelled. In other words, Scenario I takes reservoir releases as the known boundary conditions to study the impact of water management practice in the central part of the catchment under the current regulation.

Scenario II takes a different stand, aiming to reveal the 'natural state' of the catchment by modelling not only the sub-catchment $\mathrm{C}$ but also both sub-catchments in the upper streams. In this scenario, all reservoirs in the upper streams are modelled (by models A and B) as natural lakes with no flow control imposed. The same model $\mathrm{C}$ is used as in scenario I, but instead of being driven by the historical observed flow data at the two inlets, the simulation of model $\mathrm{C}$ takes inputs from the simulation of model $\mathrm{A}$ and $\mathrm{B}$ at the inlets. The same representation of water abstraction in sub-catchment $\mathrm{C}$ is adopted. In other words, scenario II is not a 'pure' natural flow simulation as it considers the water abstraction; however, it does offer an insight of the impact of flow regulation. 


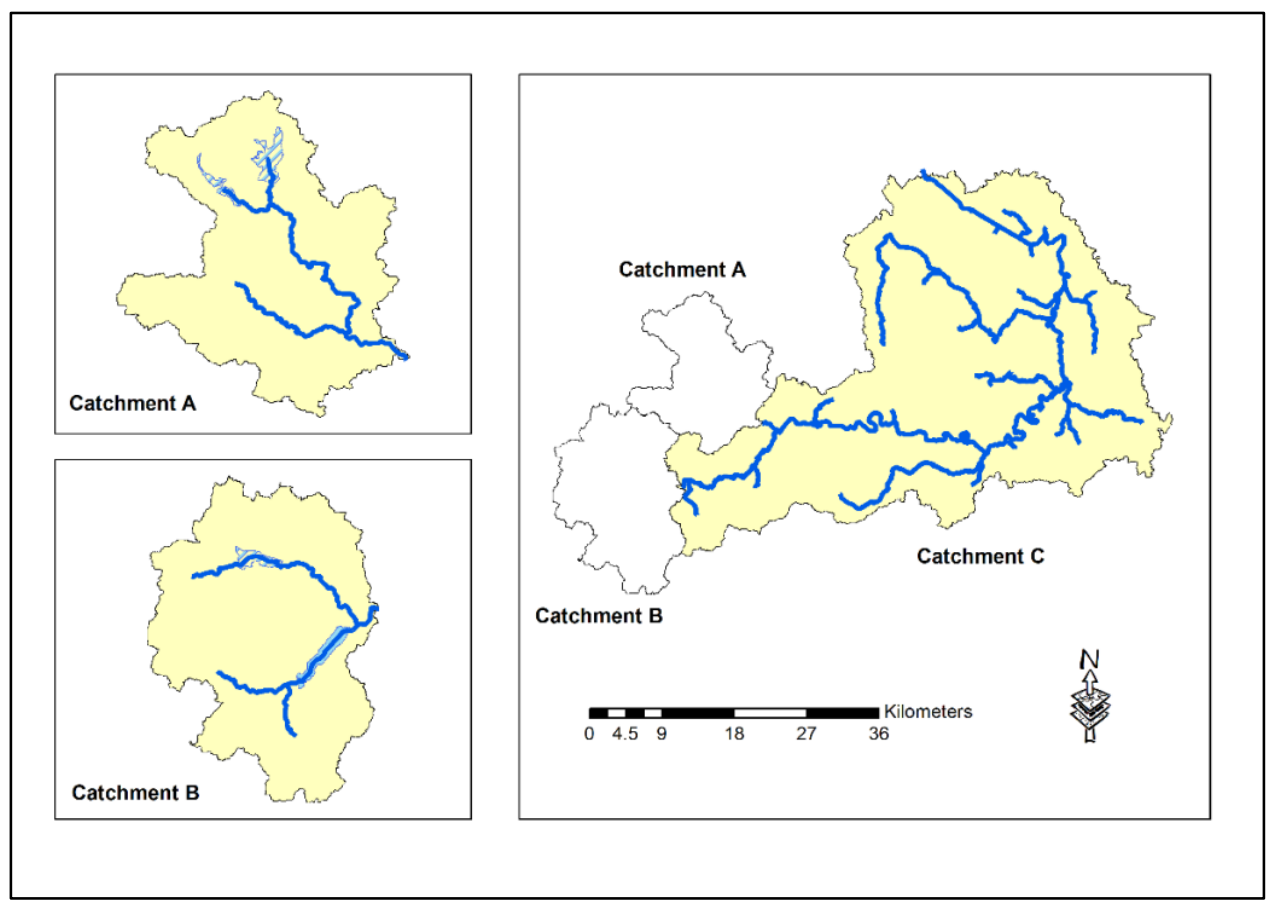

Fig. 3-6 The main and the upstream catchments of Dee River basin

\subsubsection{Model calibration and validation}

The SWAT model is calibrated and validated using the Sequential Uncertainty Fitting algorithm, SUFI2 (Abbaspour et al., 2004, 2007). In SUFI2, the uncertainties of input parameters are presented as uniform distribution. In this algorithm, the uncertainty of the model output is measured by $95 \%$ prediction uncertainty (95PPU) which is computed at the $97.5 \%$ and $2.5 \%$ levels of the cumulative distribution of output variables obtained through the Latin hypercube sampling (Abbaspour et al., 2007). The concept of the uncertainty analysis of the SUFI2 algorithm is graphically illustrated in Fig. 3-7.

As in Fig. 3-7, a single parameter value (revealed by point, Fig. 3-7a) will result in a single model response. Meanwhile, promulgation of the uncertainty in a parameter will cause the 95PPU illustrated by the shaded region (Fig. 3-7b) and as the uncertainty of parameter increase, the output uncertainty will also increase (Fig. 3-7c). In the case of Fig. 3-7d, the range of parameter must be changed in a proper direction, and if this range already corresponds to the limits of physically meaningful values, 
then the problem is not one of parameter calibration and the conceptual model must be re-examined (Abbaspour et al., 2007).

The SUFI2 algorithm starts by assuming a considerable uncertainty of parameter, thus, that the measured data initially fall within the 95PPU, then decrease this uncertainty in steps until two rules are satisfied (Abbaspour et al., 2007):

(1) The 95PPU band brackets 'most of the observations'; and

(2) The average distance between the upper (at 97.5\% level) and the lower (at $2.5 \%$ level) parts of the 95PPU is 'small'.

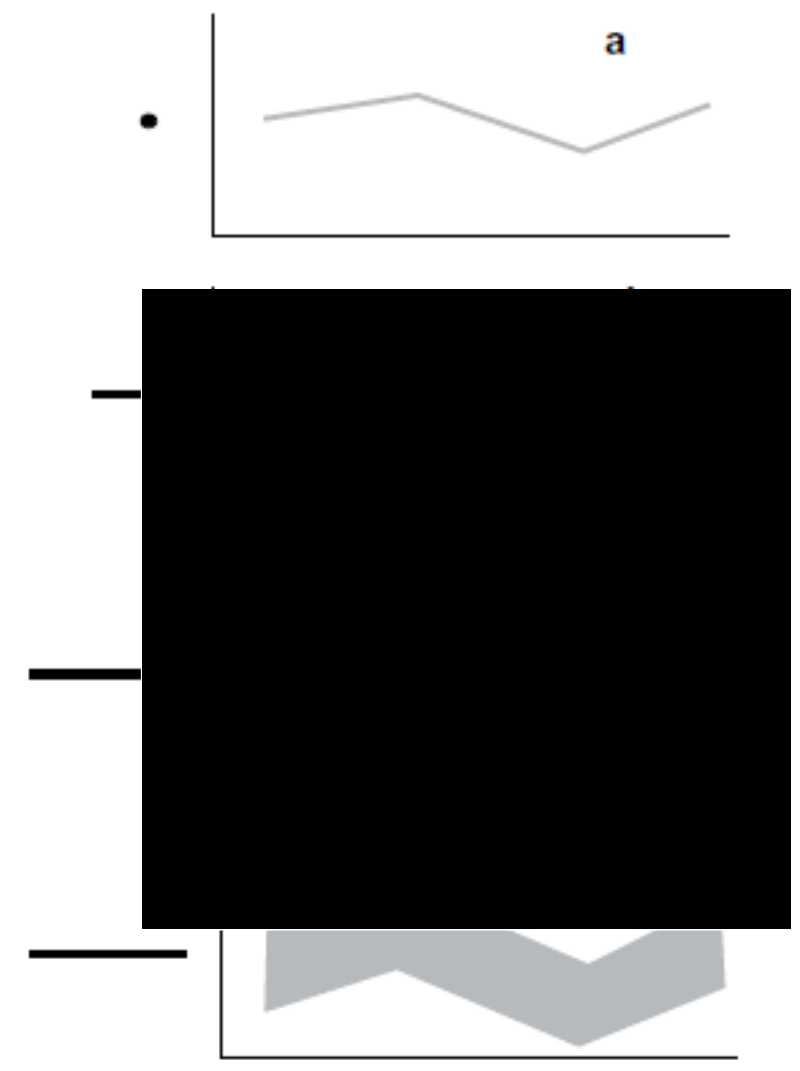

Fig. 3-7 Graphical representation of the relationship between the uncertainty of parameter and the prediction uncertainty of SUIFI2 algorithm (After Abbaspour et al., 2007)

The quantification of the two rules is some-what problem dependent. If measurements are of high quality, then $80-100 \%$ of the measured data should be bracketed by the 95PPU, while a low-quality data may contain many outliers and it may be sufficient to account only for $50 \%$ of the data in the 95PPU (Abbaspour et al., 2007). For the 
second rule, it is required that the average distance between the lower and the upper 95PPU be smaller than the standard deviation of the measured data. While looking for the smallest possible uncertainty band, a balance between the two rules ensures bracketing most of the data within the 95PPU. The two measures are used the above to quantify the strength of calibration and accounting of the combined parameter, model and input uncertainties. For detailed information about this algorithm, readers can refer to Abbaspour et al. (2004, 2007).

The goodness of fit is quantified using the Nash-Sutcliffe Efficiency Index NSE (Nash and Sutcliffe, 1970), Determination Coefficient $\mathrm{R}^{2}$ and Percent of Bias PBIAS as defined by equations:

$$
\begin{gathered}
N S E=1-\frac{\sum_{t=1}^{T}\left(Q_{o, t}-Q_{s, t}\right)^{2}}{\sum_{t=1}^{T}\left(Q_{o, t}-\overline{Q_{o}}\right)^{2}} \\
R^{2}=\left[\frac{\sum_{t=1}^{T}\left(Q_{o, t}-\overline{Q_{o}}\right)\left(Q_{s, t}-\overline{Q_{s}}\right)}{\sum_{t=1}^{T}\left[\left(Q_{o, t}-\overline{Q_{o}}\right)^{2}\right]^{0.5} \sum_{t=1}^{T}\left[\left(Q_{s, t}-\overline{Q_{s}}\right)^{2}\right]^{0.5}}\right]^{2} \\
P B I A S=\left[\frac{\sum_{t=1}^{T}\left(Q_{s, t}-Q_{o, t}\right)}{\sum_{t=1}^{T} Q_{o, t}}\right] \times 100 \%
\end{gathered}
$$

where $Q_{o, t}$ is the observed data value at time $t$ and $Q_{s, t}$ is the simulated data value at time t. It was found that the natural process plays a secondary role and surface water abstractions have a considerable impact on the river flow regime. Historical flow records at six river gauge stations are used to measure the performance of model $\mathrm{C}$ whereas that of model A and B are checked again the records at the two inlets as revealed in Fig. 3-8. Table 3-3 shows the river gauges' station used in the model calibration and validation. Table 3-4 summaries the calibration and validation of three models. 


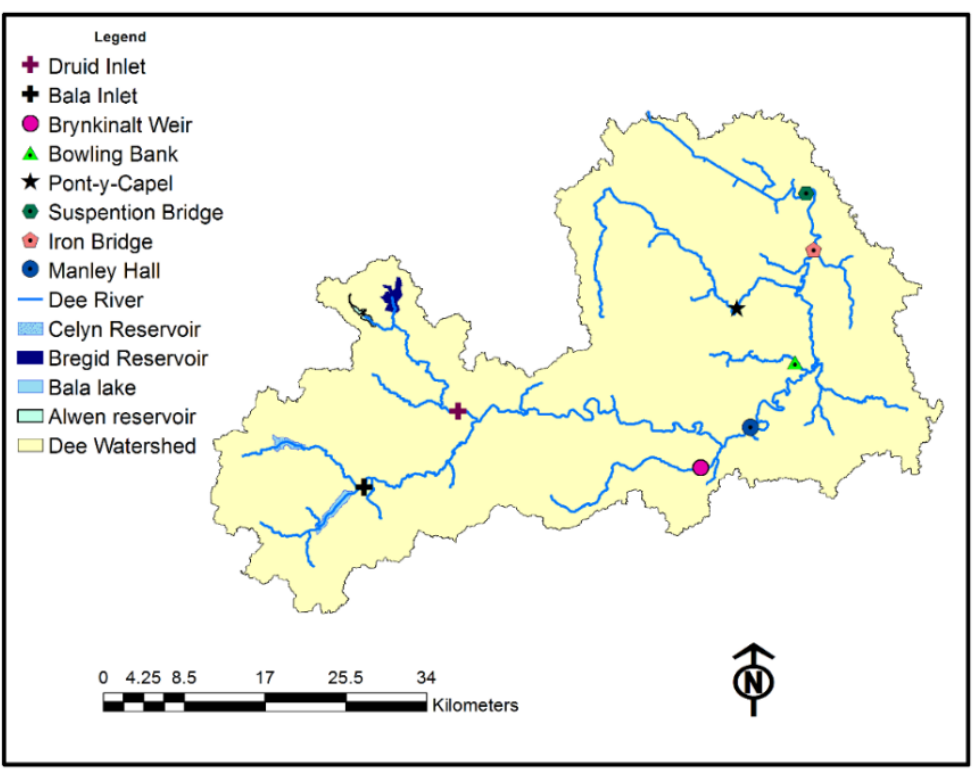

Fig. 3-8 Locations of the main inlets and the river gauge stations in Dee River basin

Table 3-3: The river gauge stations utilised in the calibration and validation of the hydrological model (Source: Centre for Ecology and Hydrology, 2015)

\begin{tabular}{|c|c|c|c|}
\hline Station Name & Latitude & Longitude & General Description \\
\hline Manley Hall & 52.966 & -2.972 & $\begin{array}{l}\text { A symmetrical compound Crump } \\
\text { weir. }\end{array}$ \\
\hline Chester Iron bridge & 53.134 & -2.873 & $\begin{array}{l}\text { Station utilises Ultra-Sonic to derive } \\
\text { flow. }\end{array}$ \\
\hline $\begin{array}{l}\text { Chester Suspension } \\
\text { Bridge }\end{array}$ & 53.187 & -2.884 & Ultra-Sonic flow gauge. \\
\hline $\begin{array}{l}\text { Alyn at Pont-y- } \\
\text { Capel }\end{array}$ & 53.079 & -2.994 & $\begin{array}{l}\text { A symmetrical compound crump } \\
\text { weir. }\end{array}$ \\
\hline $\begin{array}{l}\text { Clywedog at } \\
\text { Bowling Bank }\end{array}$ & 53.027 & -2.903 & Simple Crump profile weir. \\
\hline $\begin{array}{l}\text { Ceiriog at } \\
\text { Brynkinalt Weir }\end{array}$ & 52.928 & -3.050 & Compound broad-crested weir. \\
\hline Alwen at Druid & 52.981 & -3.431 & Reservoirs (Brenig and Alwen) \\
\hline Bala Lake & 52.908 & -3.577 & $\begin{array}{l}\text { Reservoir(s) in catchment affect } \\
\text { runoff. }\end{array}$ \\
\hline
\end{tabular}


Table 3-4: The calibrations and validations of the three models A, B and C in the

\begin{tabular}{|c|c|c|c|c|c|}
\hline Scenario & \multicolumn{3}{|c|}{ I ('real-life' case) } & & II ('ne \\
\hline Sub-catchments & $\bar{A}$ & $\mathbf{B}$ & $\mathrm{C}$ & $\mathbf{A}$ & \\
\hline Models & $\begin{array}{l}\text { Measured flow at } \\
\text { the outlet is used } \\
\text { to drive Model C }\end{array}$ & $\begin{array}{l}\text { Measured flow at } \\
\text { the outlet is used } \\
\text { to drive Model C }\end{array}$ & Model C & $\begin{array}{c}\text { Model A where } \\
\text { reservoirs are } \\
\text { modelled as natural } \\
\text { lakes }\end{array}$ & $\begin{array}{r}\mathrm{Mc} \\
\mathrm{re} \\
\bmod \end{array}$ \\
\hline Calibration Period & N/A & N/A & $(1995-2000)$ & (1995-2000) & \\
\hline Validation Period & N/A & N/A & $(2001-2003)$ & $(2001-2003)$ & \\
\hline Simulation & N/A & N/A & $\begin{array}{l}\text { River flow + } \\
\text { water yield. }\end{array}$ & $\begin{array}{c}\text { Daily flow as one } \\
\text { of the main inlet to } \\
\text { model C }\end{array}$ & $\begin{array}{r}\text { Daily } \\
\text { the }\end{array}$ \\
\hline $\begin{array}{l}\text { Gauge stations } \\
\text { used to measure } \\
\text { the performance }\end{array}$ & N/A & N/A & $\begin{array}{l}\text { Stations no. } \\
(1,2,3,4,5 \\
\text { and } 6)\end{array}$ & Alwen at Druid & \\
\hline
\end{tabular}




\subsection{Results and discussion}

In this study, the Dee River watershed was subdivided into 57 sub-basins and 1074 HRUs. The watershed parameterisation and the model input are derived using the ArcSWAT interface (SWAT 2012, revision 627) in ArcMap 10.2 environment, which offers the graphical support to the disaggregation scheme and allows the construction of the model input from digital maps. The underlying data sets required to develop the model input are: topographical, land use, soil, and climatic data. The SWAT model is constructed for the study area based on daily time step with 3 years warm-up period (1992-1994) and for the calibration period of 1995-2000, and validation period of 2001-2003 (with a warm-up period of 1992-2000). Abbaspour (2013) recommended a warm-up period of 2-3 years for hydrological models with the SWAT software.

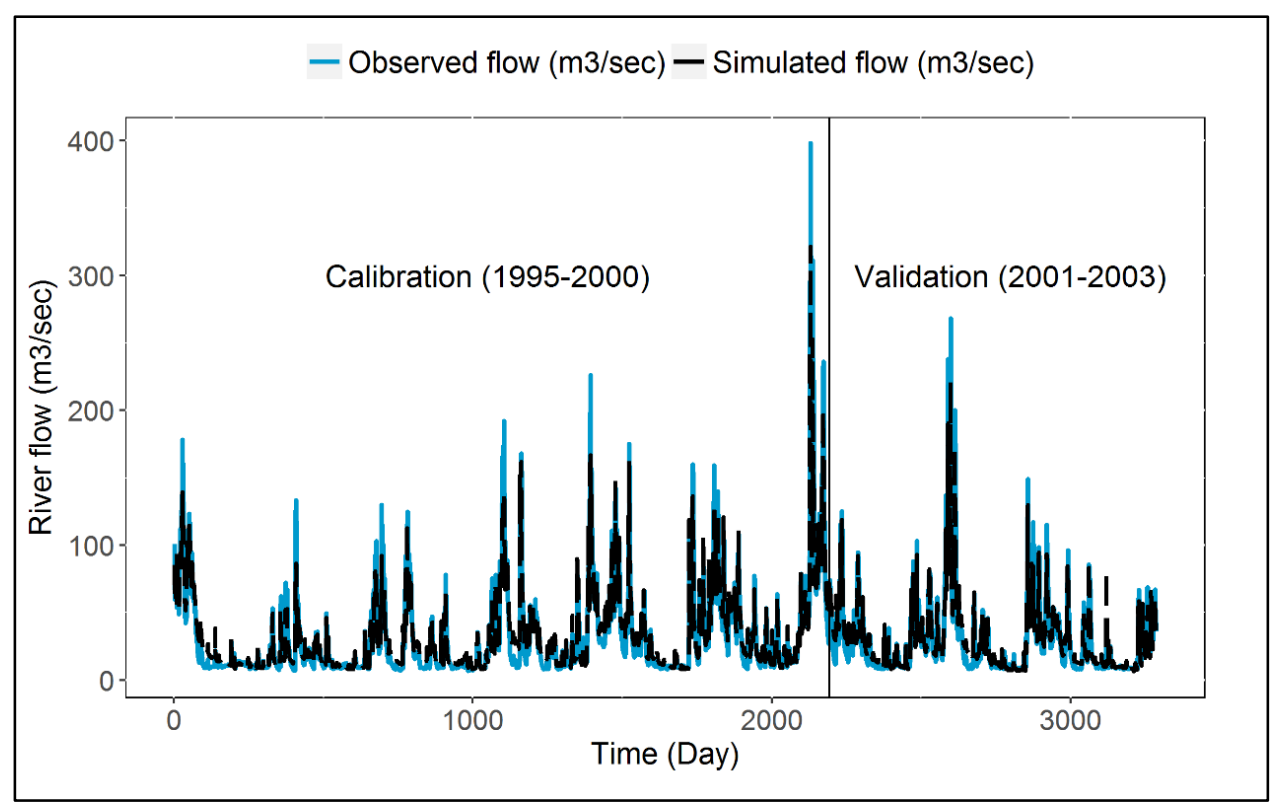

Fig. 3-9 Flow hydrograph at Manley Hall station over both the calibration and the validation periods (Catchment $\mathrm{C}$ )

The model was calibrated based on the daily measured discharge at six stations for the main catchment (Catchment C, Fig. 3-6) and one station for catchment B and one for catchment A. The comparison between the calibrated stream flow and observations using observed rain gauges at 8 locations revealed a good model performance in term of statistical performance of $\mathrm{R}^{2}$, NSE and PBIAS as illustrated in table 3-5 and the hydrograph of observed and simulated flow for Manley Hall station shown in Fig. 3-9 
(Catchment C), Druid station in Fig. 3-10 (Catchment A) and Bala station in Fig. 3-11 (Catchment B).

Table 3-5: Calibration (1995-2000) and validation (2001-2003) of the SWAT model

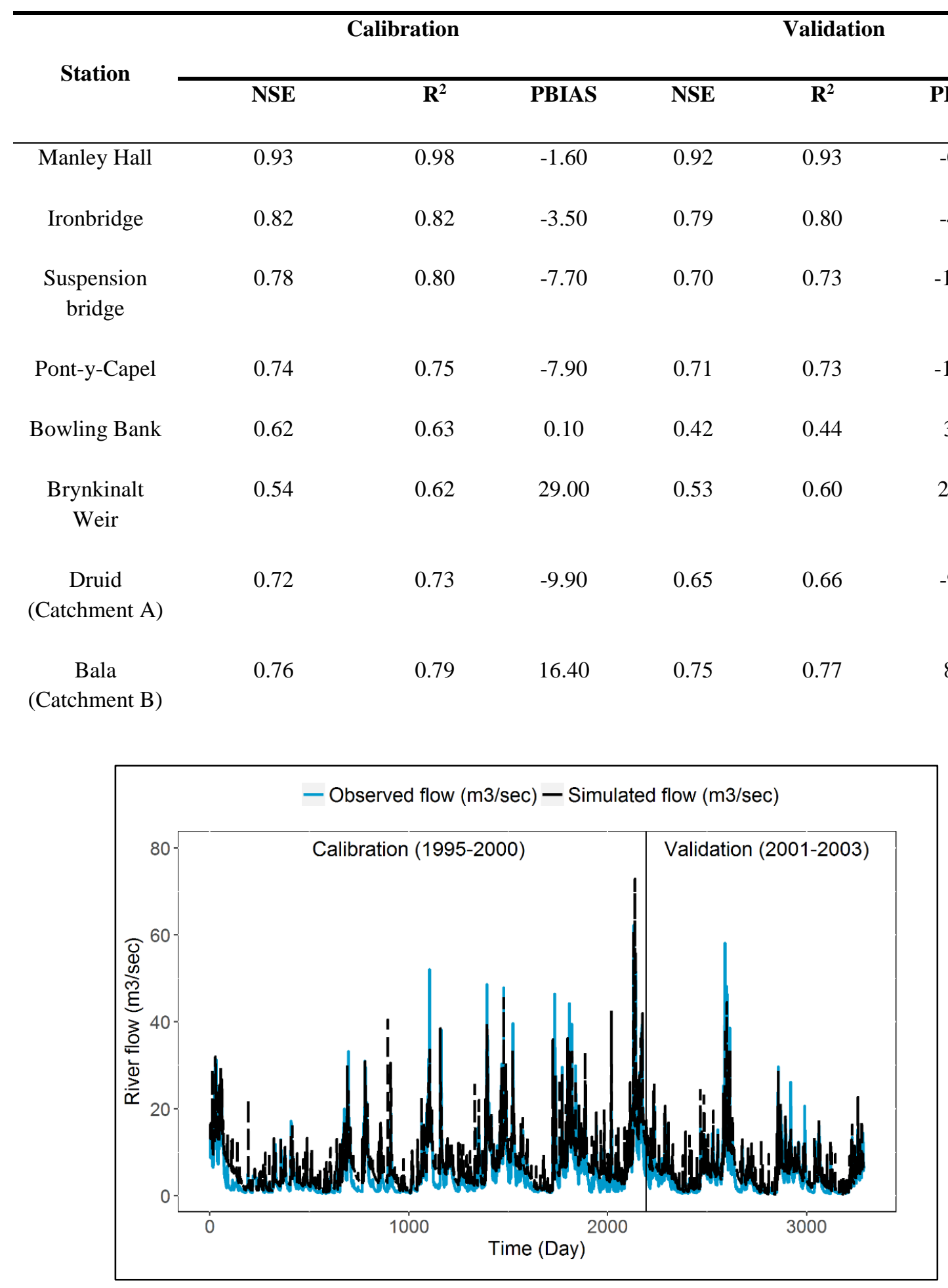

Fig. 3-10 Flow hydrograph at Druid station over both the calibration and the validation periods (Catchment A) 


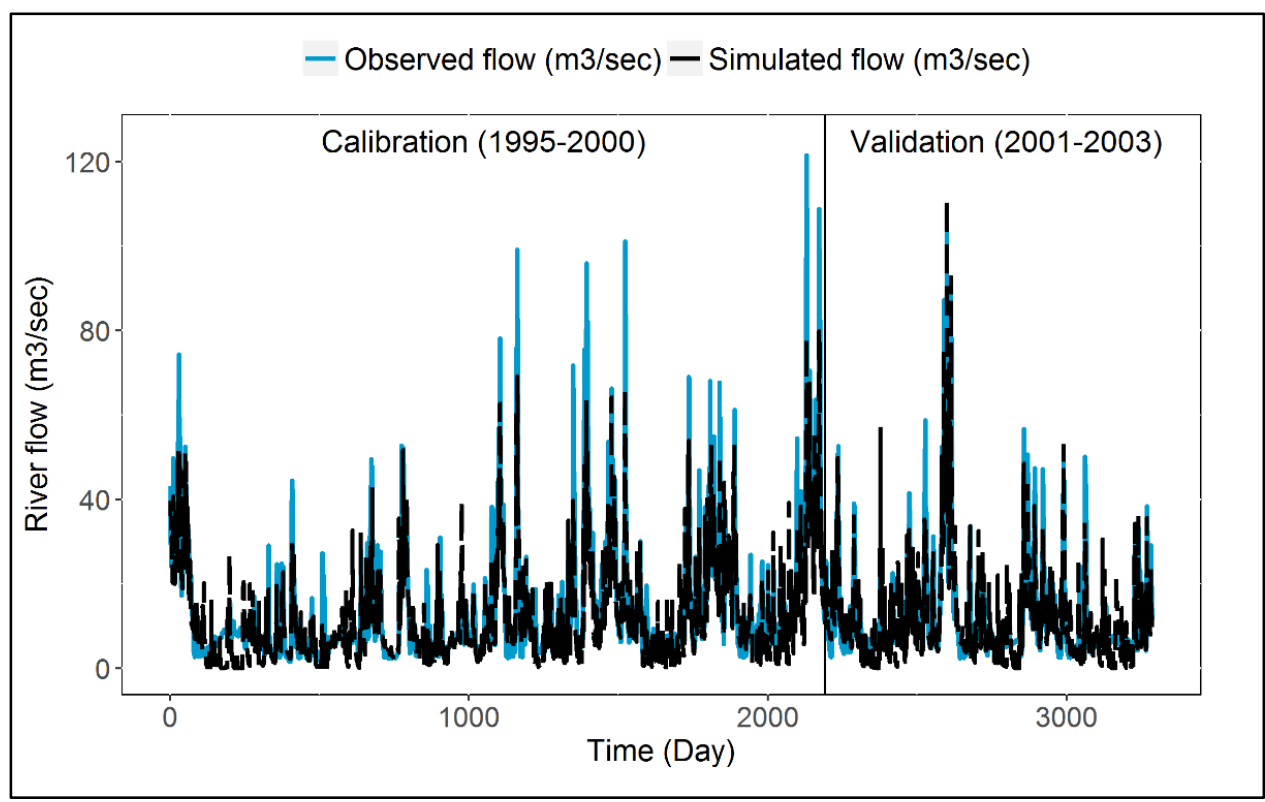

Fig. 3-11 Flow hydrograph at Bala station over both the calibration and the validation periods (Catchment B)

While, in general, the SWAT model of the Dee River basin reveals a good performance in most stations, some other stations such as Brynkinalt Weir and Bowling Bank show a low performance. The likely reasons are:

1) The way of rainfall input in the SWAT model which takes only one rain gauge station nearest to the centroid of a sub-basin. This crude estimations of rainfall might have an impact on model performance especially in mountainous areas and when the size of sub-basin is large (i.e. Brynkinalt Weir); and

2) The modelling of highly-regulated river basins is a challenging process because the natural processes play a secondary role in the catchment and there is a need of a proper approach to estimate water withdraws (i.e. Bowling Bank).

The precipitation impact on the model performance outputs will be fully explored in the next chapter with the focus on Brynkinalt Weir. Table 3-6 shows the significant SWAT parameters with their typical ranges that used in the calibration process (SUFI 2) that mostly control the streamflow. 
Table 3-6: Main SWAT parameters used in the calibration process

\begin{tabular}{lll}
\hline Parameters & Description & Typical range \\
\hline CN2 & SCS runoff curve number & $35-98$ \\
SOL_AWC & Available water capacity of the soil layer & $0-1$ \\
ESCO & Soil evaporation compensation factor & $0-1$ \\
HRU_SLP & Average slope steepness & $0-1$ \\
OV_N & Manning's "n" value for the overland flow & $0.008-0.6$ \\
SLSUBBSN & Average slope length & $10-150$ \\
GWQMN & Threshold depth of water in the shallow aquifer required for & $0-5000$ \\
GW_REVAP & Groundwater "revap" coefficient & $0.02-0.2$ \\
REVAPMN & Threshold depth of water in the shallow aquifer for "revap" to & $0-500$ \\
SOL_K & Occur (mm) & $0-2000$ \\
ALPHA_BF & Baturated hydraulic conductivity (mm/hr) & $0-1$ \\
GW_DELAY & Groundwater delay (days) & $1-450$ \\
SOL_BD & Moist bulk density & $0.9-2.5$ \\
CH_S2 & The average slope of the main channel & $-0.001-10$ \\
CH_N2 & Manning's "n" value for the main channel & $-0.01-0.3$
\end{tabular}

The SUFI 2 algorithm in the SWAT-CUP software package (Abbaspour, 2013) is used for the calibration and validation of the daily SWAT model in the Dee River basin at eight river gauges stations. A total of 12-15 SWAT parameters were selected for the calibration for the streamflow prediction with 500 iterations at each river station based on earlier studies and SWAT documentation (e.g. Abbaspour et al., 2015). For time-consuming large-scale models, SUFI 2 was found to be reasonably effective (Yang et al., 2008).

Dotty plots were used here at Brynkinalt Weir station (as an example) to illustrate the sensitivity of the model parameters utilised for the calibration of the SWAT (Fig. 312). The results of the model run with NSE as an objective function during the calibration process. 

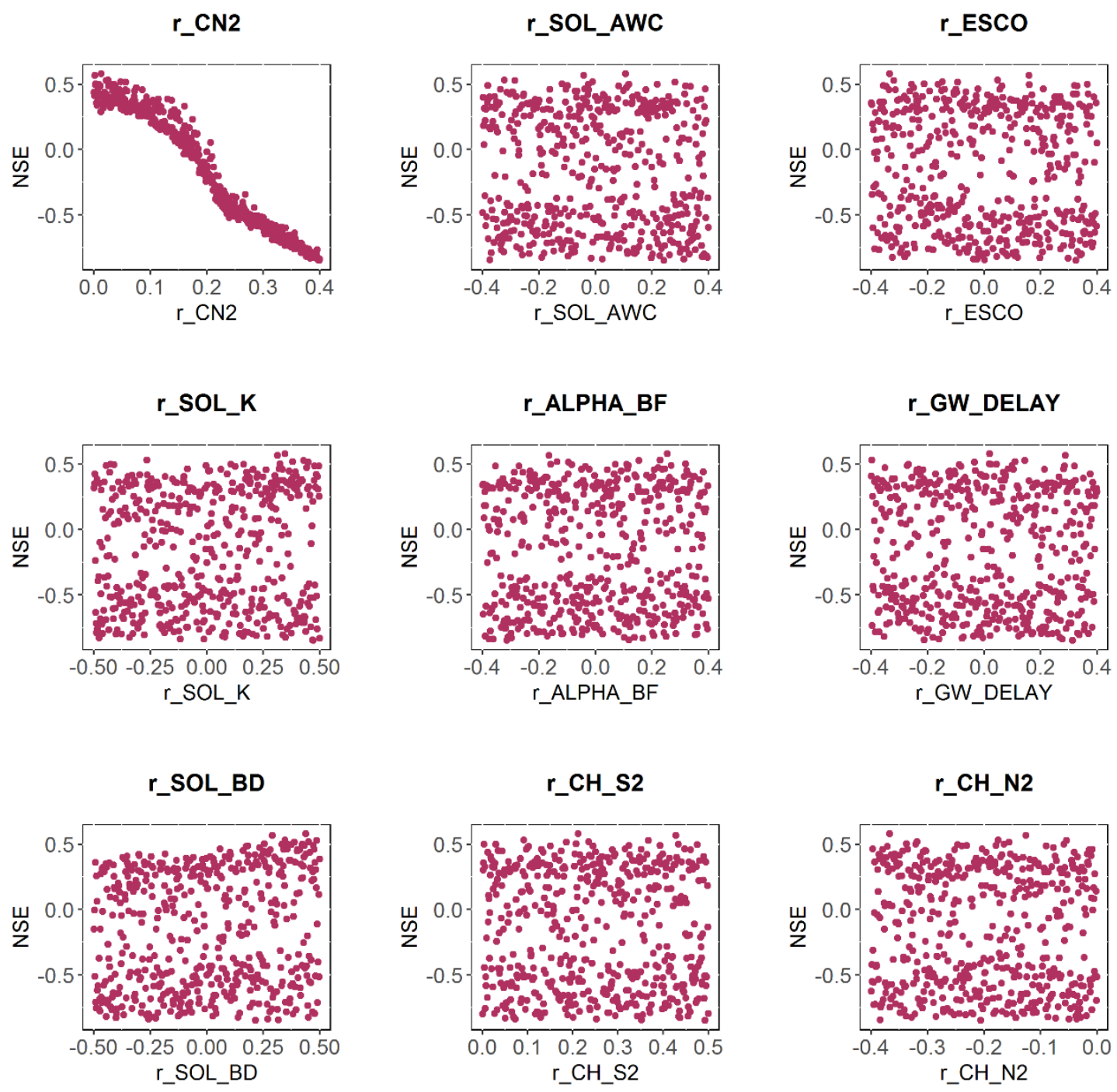

r_GWQMN
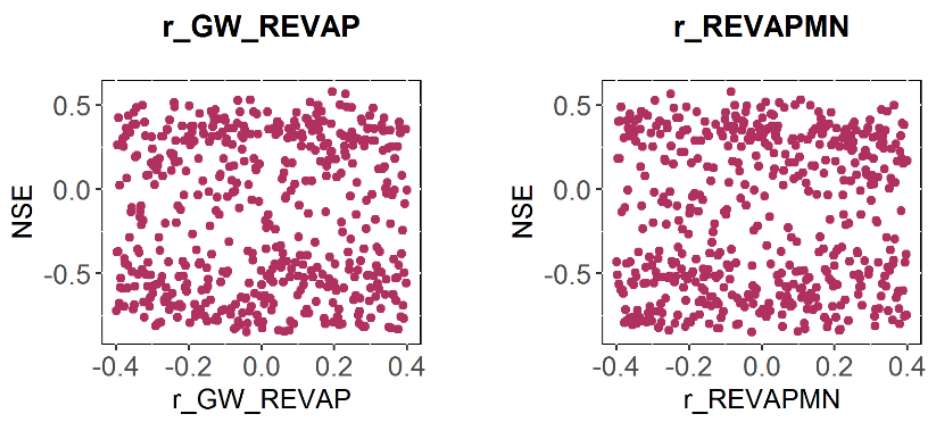

Fig. 3-12 Dotty plots with the objective function of NSE coefficient against each relative change of SWAT parameters at Brynkinalt Weir station 
When a sharp and clear peak is detected for a parameter, it can be treated as the parameter with the highest likelihood. In a similar manner, the insensitive parameters were gotten by diffused peak characterised by cumulative distributions which in turn signalise that a parameter is less proficient for the river flow prediction in Brynkinalt Weir station. The sensitivity analysis of model parameters at Brynkinalt Weir revealed that the lower performances might be instigated by structural inadequacies in model components.

Table (3-7) reveals details of the parameters being applied for calibration of the SWAT model in the Brynkinalt Weir station. The results of calibration have confirmed that all the 12 sensitive parameters are considered to apply to surface runoff, groundwater, soil properties and channel routing. The results of global sensitivity analysis with the t-test in Table (3-7) and Fig. 3-12 show that the most sensitive parameters are Curve number (CN2) followed by Groundwater 'revap' coefficient (GW-Revap).

Table 3-7: Sensitivity of SWAT parameters included in the final calibration and t-Stat and p-values at Brynkinalt Weir station

\begin{tabular}{cccccc}
\hline Parameters & Min. value & Max. value & Fitted value & t-Stat & P-Value \\
\hline r*_CN2 & 0.00 & 0.80 & 0.02640 & -39.84 & 0.00 \\
r_GW_REVAP & -0.40 & 0.40 & -0.08560 & -2.35 & 0.02 \\
r_SOL_BD & -0.50 & 0.50 & 0.43700 & 1.49 & 0.14 \\
r_SOL_K & -0.50 & 0.50 & 0.34700 & 1.12 & 0.26 \\
r_SOL_AWC & -0.40 & 0.40 & 0.10640 & -1.07 & 0.28 \\
r_GWQMN & -0.40 & 0.40 & 0.19440 & -0.82 & 0.41 \\
r_ALPHA_BF & -0.40 & 0.40 & 0.25520 & -0.62 & 0.53 \\
r_CH_N2 & -0.40 & 0.00 & -0.07800 & 0.56 & 0.58 \\
r_ESCO & -0.40 & 0.40 & -0.33520 & 0.48 & 0.63 \\
r_REVAPMN & -0.40 & 0.40 & -0.19600 & 0.11 & 0.91 \\
r_GW_DELAY & -0.40 & 0.40 & 0.02000 & 0.07 & 0.94 \\
r_CH_S2 & 0.00 & 0.80 & 0.33840 & 0.03 & 0.97 \\
\hline
\end{tabular}




\subsection{Summary}

Many river basins around the world are highly-regulated with a range of water abstraction rules and regulations and several physical flow control and storage structures. Most existing hydrological models do not represent the modifications to the hydrological regimes introduced by water management such as reservoirs and water abstractions. The interactions between natural hydrological processes and changes in energy fluxes, water and storage attributable to human interventions are essential for water resources managers for the understanding of how these systems might respond to climate change among other drivers for change and the evaluation of their feedbacks to the climate system at regional scales. One such river basin is the Dee River catchment in the United Kingdom.

This study shows an integrated modelling method to include human interventions within natural hydrological systems using the SWAT model. Three daily SWAT models are set up to simulate the hydrological processes in the Dee River catchment with physical flow control and water withdrawal process explicitly represented. Two scenarios (with and without flow control) are constructed to explore the impact of management practice. The representation of the management practice such as flow control and water withdraw in the modelling process can help reveal the effect from the latter, and as such lays a foundation for further study on how various management practice can mitigate the impact of other sources such as climate change on catchment water resources management which will be discussed later.

An inversion approach was used to estimate the daily water withdrawal amount at the water abstraction points, as this amount is not available from the data collected. The estimation was based on the information from PWS companies who offer a weekly abstraction forecast to Natural Resources Wales to assist in calculating the required releases from the reservoirs. Justifiably, although this technique helps to show the interaction between flow regulation and water abstraction, it also has a certain amount of uncertainties. It can be developed by further modelling the probabilistic nature of water abstraction or by conditioning them on other variables such as temperature. 


\section{Chapter 4: Model Input Uncertainty}

A hydrological model (SWAT) was used in the previous chapter to simulate land surface processes in a highly-regulated river basin to study the intervention of natural processes and human activities within the watershed. It can be deemed as the first step for IWRM framework. Another significant issue needs to scrutinise is the uncertainty identification due to input data, i.e. rainfall. Data uncertainty arises owing to the error in measurement and data handling and limitation of data in time and space, which results in an insufficient representation of the study area.

The focus of this chapter is to examine the impact of different precipitation preprocessing methods on model calibration and the overall model performance with regards to its operational use. The same Dee River (refer to the model in chapter 3 ) is modelled to test against the three pre-processing methods of precipitation: The Centroid Point Estimation Method (CPEM), the Grid Area Method (GAM) and the GridPoint Method (GPM). Benefited from the newly produced, the high-resolution Gridded Estimate Areal Rainfall (CEH-GEAR) dataset, cross-calibration and validation are made possible. The primary focus of this chapter is the study of the impact of precipitation pre-processing on SWAT model calibration and validation as well as operational use.

\subsection{Introduction}

Precipitation is one of the vital forcing factors in hydrological modelling practices. The accuracy of precipitation input and its representation have a direct impact on the overall model performance. In the last few decades, many studies were reported to have looked into this, mainly due to the drive of quantifying modelling uncertainties where the inputs such precipitation have to be taken into account, for example (Masih et al., 2011; Schuurmans et al., 2007). 
Alongside the concerns of accuracy, the importance of spatial variability of rainfall has also been highlighted, especially over large watersheds where it is crucial to gain insight of day-to-day spatial variability of groundwater level, stream flow discharge and soil moisture content (Schuurmans et al., 2007). At smaller scales, rainfall variability also has a considerable impact on peak flow estimation (Mandapaka et al., 2009). Segond et al. (2007) reported that as the scale increases, the effect of rainfall distribution decreases and there is a transfer from the spatial variability of rainfall to catchment response time distribution as the dominant factor governing runoff generation.

The effect of various spatiotemporal resolutions of precipitation on simulated runoff has also been widely investigated by, e.g., Tetzlaff and Uhlenbrook (2005); Maskey et al. (2004) which agreed on the necessity of adopting better rain representation input in modelling structure. However, most of these studies are focused on a specific model or models because that precipitation pre-processing is often model-dependant, although many different models use specific common methods such as Thiessen polygon method.

The density of rain-gauges over the forecast watersheds is one of the key factors in succeeding forecast accuracy during an extreme event that gives rise to substantial flooding in a major metropolitan region (Looper and Vieux, 2011). Consequently, the precipitation data should be processed as spatially distributed input before being forced into hydrological models. However, measuring at every point is prohibited by the high costs associated. Caruso and Quarta (1998) reported that spatially distributed precipitation could be interpolated using a range of various techniques. Nevertheless, the complexity depends on selecting the one that best reproduces the most precise data.

Shah et al. (1996b) argued that the average of the precipitation inputs reduces the accuracy of the model's results. Under such conditions, catchment response is highly 
non-linear, which means that the response to an averaged input will differ much more from the response to a distributed input. When a single rain gauge is used to model the catchment response, the results become less accurate at both the sub-basin and basins scales, and this also affects the production of the hydrograph (Segond et al., 2007).

Spatial variability in precipitation influences on hydrological model outputs (e.g. Bell et al., 2000; Segond et al., 2007; Cole and Moore, 2008; Collischonn et al., 2008), the estimation of model parameters (Chaubey et al., 1999), the catchment response (Shah et al., 1996a; Shah et al., 1996b) and the timing of peak runoff (Singh, 1997). Schuurmans et al. (2007) state that failing to consider a satisfactorily spatial distribution of precipitation will result in errors in the values of the model parameters that will be wrongly changed to compensate for errors in the rainfall input. A large number of interpolation methods that have been introduced and employed in the literature that create the spatial distribution of precipitation based on rain-gauges measurements. These methods can generally be classified into two main groups: deterministic and geostatistical methods (Ly et al., 2011).

The first group of spatial interpolation methods for measuring precipitation, which probably is one of the most frequently used deterministic techniques, includes the Inverse Distance Weighting (IDW) and Thiessen polygon (THI), which are based on the location of the measured stations and measured values. The Arithmetic Means, the simplest spatial interpolation method, can also be used in relatively flat regions. Nevertheless, use of this technique has decreased as it does not provide demonstrative measurements of precipitation in most cases (Ly et al., 2011).

The other group of spatial interpolation methods is geostatistical techniques, establishing a discipline connecting earth sciences with mathematics (Ly et al., 2011). Kriging is an example of geostatistical techniques used in a random field to 
interpolate a variable. The geostatistical methods can create a prediction surface and offer some measurements of the accuracy of the predictions.

Dirks et al. (1998) pointed out that deterministic interpolation methods revealed a better performance than geostatistical ones. They also evaluated the performance of three interpolation methods THI, IDW and Kriging in Norfolk Island by using rainfall data of 13 rain gauges considering time steps of an hour, day, month and year. The authors have recommended the IDW interpolation method. Meanwhile, other researchers such as Nalder and Wein (1998) found that IDW had a similar error with Ordinary and Universal Kriging in the interpolation of monthly rainfall.

Thanks to its open-source strategy and easy-to-use GUI, the Soil and Water Assessment Tool (SWAT) has gained a large user base. Many studies using SWAT have endeavoured to address the issues of precipitation input such as the one by Chaplot et al. (2005). They studied the effect of rain gauge density on streamflow, sediment and nitrogen fluxes simulations in two small watersheds in the United States and they found that the use of higher rain gauge densities could lead to better simulations, especially for sediment fluxes. Jayakrishnan et al. (2005) compared annual and monthly river flows simulated by SWAT for four catchments in the U.S. using both weather radar (Next Generation Weather Radar, NEXRAD) and rain gauges. They concluded that input of areal rainfall measured by radar gave the best estimation, despite some inherent limitations, especially the accuracy at daily time scale.

While in general, most of those studies tend to agree that denser rain gauge network (hence being capable of better spatial representation of precipitation) can lead to improved model performance, there are others, however, do not share such a view. Cho and Olivera (2009) investigated the effect of the resolution of land use, soil type and rainfall data on simulating river flow in three catchments in the U.S constructing 
18 models of each basin by combining three land use categories, three soil types, and two precipitation input scenarios. The two rainfall scenarios employed were:

1) Data from all available rain gauges; and

2) Data from a single rain gauge for the whole catchment area.

It was found that all models produced comparable values of Nash-Sutcliffe efficiency indices. Their main findings were that more refined representation of spatial data might not necessarily result in improved SWAT river flow simulations in small catchments. It may well be attributed to other factors such as soil types and land use possibly being more dominating than the rainfall.

Remote-sensing-based rainfall measurements, such as those from weather radars and satellites excel in providing much more detailed spatial structures compared with gauge measurements, but in the meantime, they suffer from low accuracy as well (Zhu et al., 2014). Tobin and Bennett (2009) compared monthly river flow simulated by SWAT using precipitation data collected through satellites observations (Tropical Rainfall Measurement Mission), weather radar (NEXRAD) and rain gauges at the outlet of the two rivers in the U.S. They concluded that stream flows were better simulated using the radar data compared to the other two sources of rainfall input. This is indeed encouraging as it may support the idea that better spatial representation outweighs accuracy in some cases.

A more comprehensive account is given by Starks and Moriasi (2009) who compared streamflow simulations from a SWAT model using four different resolutions of rainfall data in three experimental catchments of different sizes. The number of rain gauges in three scenarios varied from 1 to 7 . The rainfall data obtained through weather radar, available at $4 \mathrm{~km}$ grids, were used in the fourth scenario. Their study produced satisfactorily calibrations for all four cases, even though the scenarios with higher rain gauge density and the radar-based rainfall showed relatively better river flow simulations. 
A more recent study by Masih et al. (2011) used a SWAT model to evaluate its performance using two interpolation methods: standard precipitation input and an areal precipitation input obtained through using the Inverse Distance and Elevation Weighting (IDEW) interpolation. This study found that the use of areal precipitation, obtained through the interpolation improved simulated stream flow. It is worth noting that most of those studies are based upon model simulations at a larger temporal scale, e.g., monthly or yearly, which has two significant implications:

1) The contribution of better spatial representation from using either denser gauge networks or remote sensing data might well be smoothed away; and

2) They may not fit the needs of day-to-day operational use.

Daily precipitation has a specific stochastic nature which differs from monthly rainfall (Johnson and Hanson, 1995; Ly et al., 2011). From a modeller's viewpoint, it would be more intriguing to explore how the way of model handling precipitation input can be improved across different scales.

There is another problematic aspect of conducting such assessment in an existing modelling system like SWAT attributable to their parameterisations. Discussion of the benefits, as well as the drawbacks of model parameterisation, goes beyond the scope of this chapter, readers can refer to Cheng et al. (2014); Abbaspour et al. (2007); Li et al. (2013) and Shrestha et al. (2016). An immediate impact of model parameterisation, however, is that at times model can be calibrated equally well even though they are fed with input data (such as precipitation) that apparently are of different quality. This so-called 'compensation of parametrisation' makes it challenging to identify and isolate the impact of various inputs by only considering model calibrations and their comparisons.

In this chapter, a study on the impact of various precipitation pre-processing methods is presented on model performance over Dee River basin. A most recent, high resolution, gridded rainfall dataset - the $\mathrm{CEH}$ Gridded Estimates of Areal Rainfall 
(Keller et al., 2015) is used as a reference in addition to the conventional gauged rainfall data. Three different pre-processing methods are tested including:

1) The default SWAT method which uses the rainfall value from the nearest gauge to the centroid of the (sub-)basin in question (Centroid Point Estimate Method, CPEM);

2) The gird-area method (GAM) which takes the averaged value of all grids of the GEAR dataset falling in the area of the sub-basin in question; and

3) The grid point method (GPM) which is similar to CPEM, but instead of using the value from the nearest gauge, it takes value from the grid of the GEAR dataset where the centroid of the sub-basin is located within.

Cross-calibration and validation of the combination of the three methods using both gauge data and GEAR data are conducted over the Dee river catchment (daily time step) in the UK, aiming to isolate the 'compensation' effect due to model parameterisation. The principal objectives of this chapter are:

1) To assess the impact on hydrological model performance from using various methods of rainfall pre-processing above and further give recommendation where possible;

2) To evaluate model parameterisation (via calibration) with different rainfall inputs on the overall model performance; and

3) To test the utility of the new GEAR dataset in the context of calibrating hydrological models. The in-depth discussion of the three pre-processing methods as well as the way of model calibration and cross-validation is given in the section of methodology.

\subsection{The CEH-GEAR precipitation dataset}

The Centre for Ecology and Hydrology - Gridded Estimates of Areal Rainfall (CEHGEAR), is a new precipitation dataset developed to provide reliable $1 \mathrm{~km}$ gridded estimates of daily and monthly rainfall over the UK and $3,500 \mathrm{~km}^{2}$ of catchment area in the Republic of Ireland from 1890 to 2012 (Keller et al., 2015). The rainfall 
estimates are created from the Met Office historical weather observations for the UK. The natural neighbour interpolation method (Ledoux and Gold, 2005) including a normalisation step based on average annual rainfall (AAR), was employed to create the daily and monthly precipitation over the regular $1-\mathrm{km}$ grids.

A schematic representation of the interpolation procedure used to derive the $\mathrm{CEH}$ GEAR daily and monthly $1 \mathrm{~km}$ grids is shown in Fig. 4-1. The grids are generated using the natural neighbour interpolation alongside a normalisation step based on AAR which involves two steps:

1) An initial estimate from daily gauges alone; and

2) Multiplication by a correction grid to give consistency with monthly grids that have been created from all available daily and monthly gauged data-daily and monthly.

Readers can be referred to Keller et al. (2015) for the detailed discussion of the derivation. It should be noted that weather radar data are not used in the production of the current version of CEH-GEAR although such merging would be able to improve the spatial representation of the interpolated field. This is, in part, due to the comparatively short duration available for the radar rainfall estimates (around 30 years) compared to the rain-gauge observations. Accordingly, CEH-GEAR data would have greater temporal consistency if it is solely based on rain gauge observations (Keller et al., 2015).

\subsection{Methodology}

\subsubsection{SWAT simulations}

For the study of the precipitation pre-processing, only Scenario I 'real-life' situation is considered (refer to section 3.4.2). When it comes to how the precipitation amount is represented, the default setting of SWAT uses the values from the gauge located closest the centroid of each sub-basin to represent the areal value for the sub-basin (Masih et al., 2011). To consider the orographic effects on temperature and rainfall in 
mountainous areas, SWAT makes use of the elevation bands method which allows for up to 10 elevation bands in each sub-basin that enable the model to assess the differences in snow cover and snowmelt caused by orographic variation in the rainfall and temperature. This method adjusts the regional precipitation by weighting the elevation difference between the band of the rain gauge and the other bands.

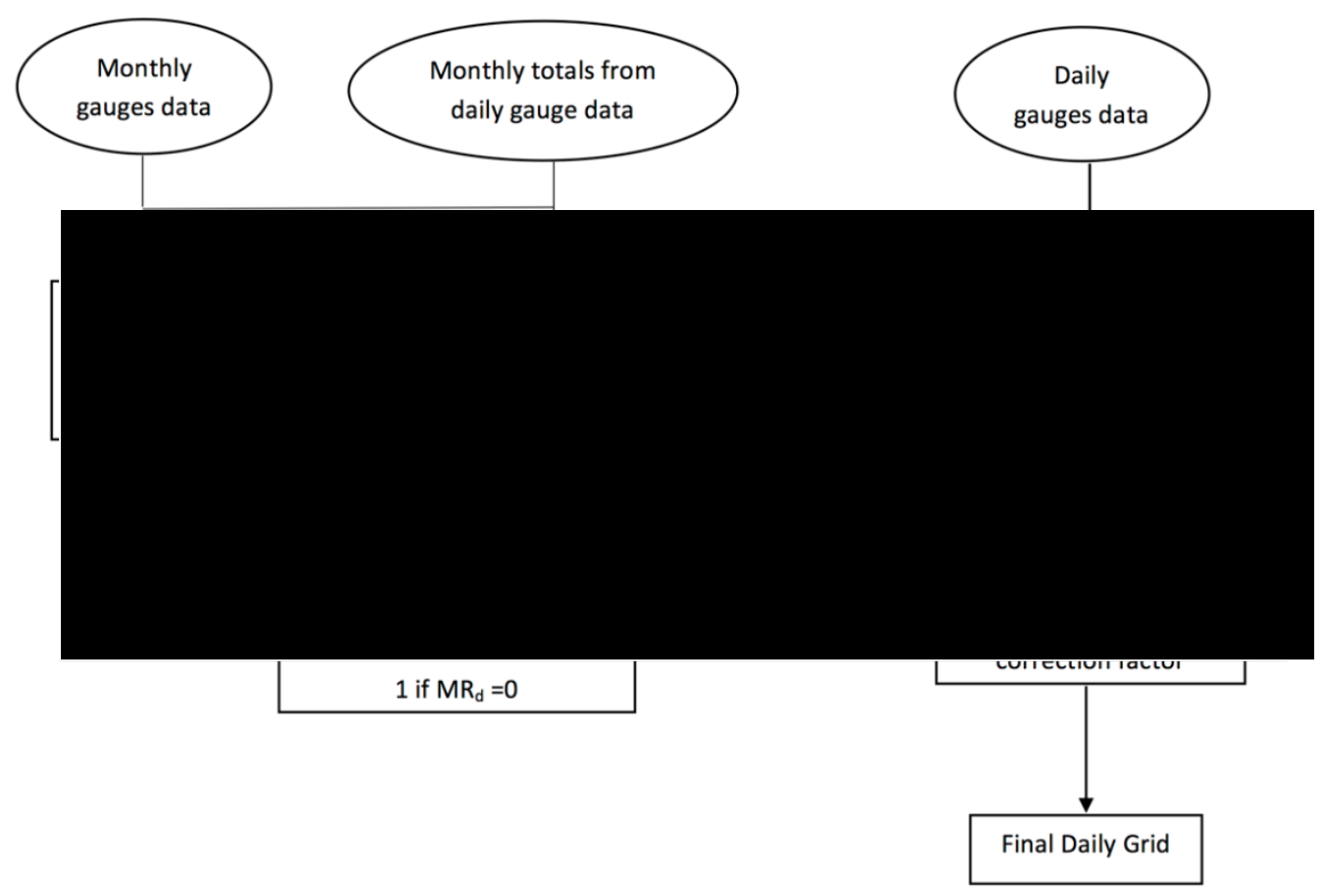

Fig. 4-1 Derivation of daily and monthly gridded rainfall estimates of CEH-GEAR (After Keller et al., 2015)

Most applications of SWAT follow this approach as there is no explicit entry in the model user interface to alter this setting conveniently. Evidently, in some cases, such treatment does not represent well the spatial variation of precipitation field hence ignoring spatial heterogeneity. One can see the impact of such treatment even without experiment because:

1) The nearest gauge value may not be able to precisely estimates the rainfall amount at the centroid; and 
2) Even if precipitation is accurately estimated at the centroid, this centroid value may not be able to represent the areal value of the sub-basin in question.

However, even using such crude estimate of sub-basin precipitation, some applications of SWAT are reported to work well. The reason lies in two folds: on the one hand, a denser gauge network and/or less intermittent rainfall events can mitigate the poor spatial representation of the model; on the other, model parameterisation can also 'compensate' (Starks and Moriasi, 2009). This, in fact, inspires this study as it is hoped to isolate the impacts of pre-processing techniques from the two factors mentioned, by applying cross-calibration and validation to separate model parameterisation.

\subsubsection{Precipitation pre-processing methods}

As previously listed, there are two types of precipitation input data used in this study. First, the measured rainfall at the 13 rain-gauges in the Dee river basin has been aggregated temporally into daily and monthly respectively. The missing data gaps in gauge observations are filled by using the IDW method. Second, the CEH-GEAR data (at $1 \mathrm{~km}$ spatial resolution) are taken without any further data screening and gapfilling operations.

The following three methods to pre-process the precipitation data are applied before using them to represent (sub-) basin areal values in SWAT:

1) The centroid point estimate method (CPEM): this is the default method used by SWAT which estimates the areal precipitation of a sub-basin utilising the rainfall at the gauge closest to the centroid of the sub-basin (see Fig. 4-2a). Only gauge data are used in this case for every sub-basin;

2) The grid-area method (GAM): this method 'cuts off' the target sub-basin area out of the GEAR grids and takes the average of all values of the grids that either is entirely within the area or intersect with it (Fig. 4-2b); and 
3) The grid-point method (GPM): this method again uses the GEAR dataset except that instead of taking the average of the intersecting areas, it estimates the value at the centroid of the target sub-basin by interpolating the values of GEAR grids nearby (within a 1-km search radius) using the IDW method (see Fig. 4-2c). The estimated centroid value is then used to represent the areal precipitation over the target sub-basin as done in CPEM.

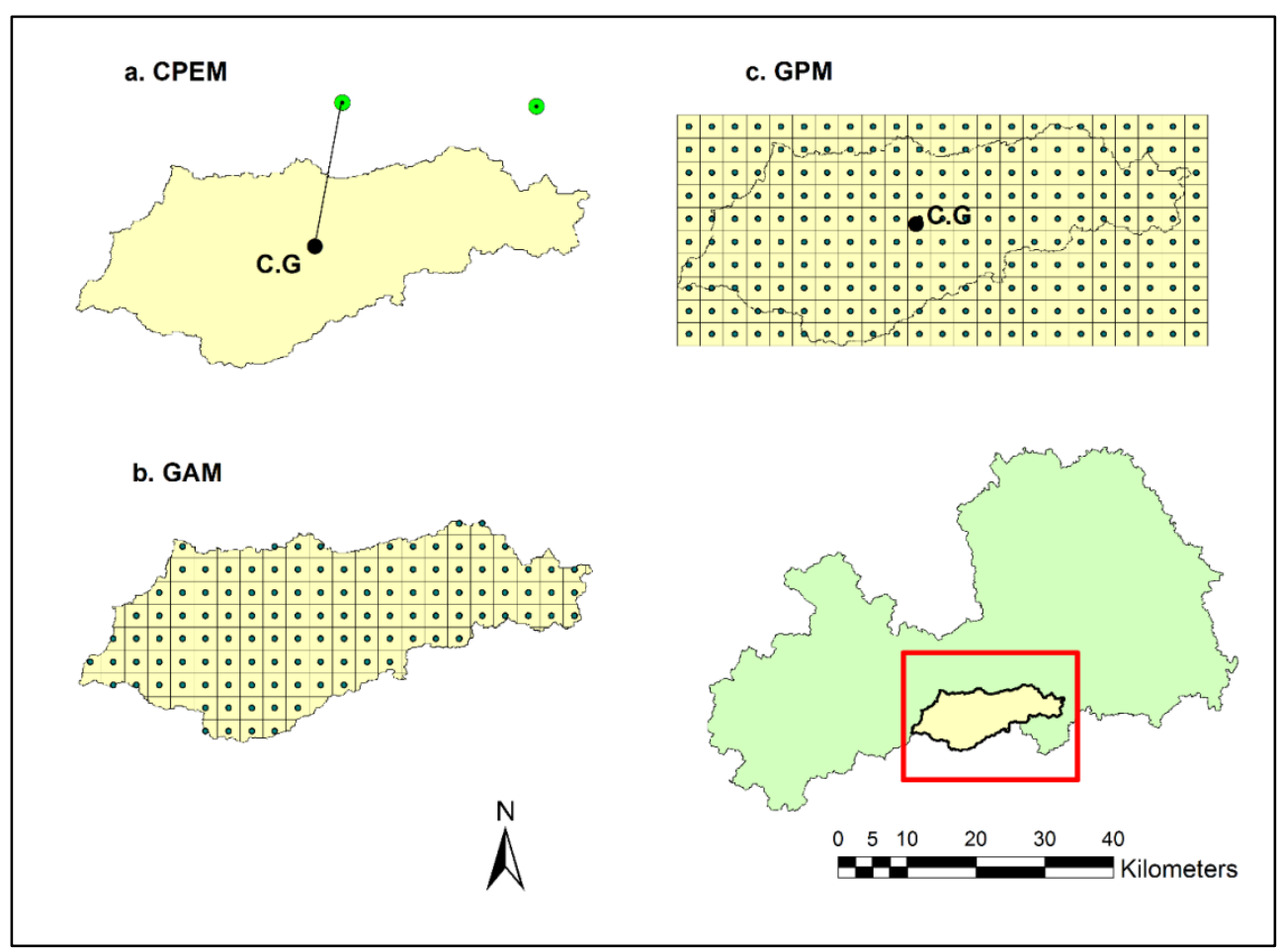

Fig. 4-2 Methods of precipitation pre-processing for a selected sub-basin in Dee River basin

Inverse Distance Weighting interpolation (IDW) computes values at un-sampled points by the weighted average of observed data at surrounding points. Accordingly, this can be defined as a distance reverse function of each point from nearby points (Teegavarupu and Chandramouli, 2005). The values at un-sampled points can be determined by using a linear combination of values at a known sampled point. IDW depends on the theory that the unknown value of a point is more affected by closer points than by points further away. The weight can be computed by: 


$$
\lambda_{i}=\frac{\frac{1}{\left|D_{i}\right|^{d}}}{\sum_{i=1}^{n s} \frac{1}{\left|D_{i}\right|^{d}}}, d>0
$$

where $D i$ is the distance between sampled and un-sampled points. The $d$ parameter is specified as a geometric form for the weight while other specifications are possible. This specification implies that if the power $d$ is larger than 1, the so-called distancedecay effect will be more than proportional to an increase in distance, and vice versa (Ly et al., 2011). Hence, small power $d$ tends to give estimated values as averages of in the neighbourhood, while large power $d$ tends to give larger weights to the nearest points and increasingly down-weights points further away (Lu and Wong, 2008).

Using a power value of 2 for daily and monthly time steps, 3 for hourly and 1 for yearly would appear to minimise the interpolation errors (Dirks et al., 1998). Moreover, this power d usually sets to 2, following Lloyd (2005) and hence inverse square distances are used in the estimation. Consequently, a power value of 2 was adopted for IDW in this study.

In a nutshell, the three proposed methods utilise both the gauge measurements (CPEM) and the CEH-GEAR dataset (for GAM and GPM). It should be noted that the CEH-GEAR data are also derived from gauge measurements that have been further gridded by applying natural neighbour interpolation. Consequently, to a certain degree, the GAM method effectively resembles the common Thiessen method which obtains areal rainfall using the underlying gauge measurements averaged over the polygons.

However, there are still some subtle differences which are:

1) The Thiessen polygon method is nearest neighbour interpolation whereas the GEAR data are derived from using the natural neighbour interpolation; and 
2) They may not use the same set of rain gauges and more sophisticated approaches of errors corrections have been applied to produce the GEAR dataset.

Nevertheless, in terms of accounting for spatial heterogeneity, the GAM method should be the best choice followed by GPM while the default method CPEM falls behind. Thus, it is hypothesised that correspondingly, models calibrated using the techniques are expected to rank in the same order regarding their performances.

\subsubsection{Cross-calibration and validation}

The standard approach to setting up SWAT models is mostly followed for the study area. For the Dee catchment, the two upper-stream sub-basins are not modelled so as to avoid the complexity of representing reservoir regulation, instead, the releases from the four water supply reservoirs and the lake (measured at the two inlets: Druid and Bala) are used as the boundary condition for the main part of Dee River which constitutes the main study area.

Rainfall data from the 13 gauges are used to construct the CPEM time series from 1995 to 2003 whereas the other two-time series produced from the CEH-GEAR dataset utilising the GAM and GPM methods respectively are also generated for the same period. A daily SWAT model fed with three rainfall time series is then calibrated over 1995-2000 and validated of 2001-2003. The SWAT model is calibrated and validated using the Sequential Uncertainty Fitting algorithm - SUFI2 (Abbaspour et al., 2004, 2007).

A cross-calibration and validation approach is used to isolating the impact of model parameterisation concerning different precipitation pre-processing schema. This means that there are three (3) calibrated models for each catchment, i.e., models that are calibrated using the three pre-processed rainfall time series based on CPEM, GAM and GPM methods. These three models are then validated using three different 
rainfall time series as well. Therefore, in the end, there are nine (9) simulations assessed during the validation stage.

\subsection{Results and discussion}

\subsubsection{Comparison of point/grid precipitation and spatial distributions}

As previously mentioned, the CEH-GEAR dataset is derived from rain gauge observations with extra quality control measure before being interpolated onto the regular grids. It is therefore expected to see a good agreement between the gauge observed precipitation values and the values from the grid of GEAR dataset that is at (nearly) the same location of the gauge. The determination coefficient and root mean square error are used to evaluate CEH-GEAR rainfall product. Root mean square error can be computed as follow:

$$
R M S E=\sqrt{\frac{\sum_{i=1}^{n}\left(P_{m}-P_{o}\right)^{2}}{n}}
$$

where $P_{m}$ modelled rainfall and $P_{o}$ is the observed one.

The data screening has been conducted on the precipitation from rain gauges for the inspecting for errors and correcting them before doing data analysis. The screening involves checking raw data, recognising outliers and dealing with missing data. The precipitation data also is being subjected to rigorous quality control by the British Atmospheric Data Centre (BADC) and the Met Office.

Daily rainfall from the grids closest to the 13 gauges locations in Dee catchment is extracted from the GEAR dataset and then compared with the time series of the 13 gauges values. As expected, the time series are well-perfect matched at the 13 locations as seen in Table 4-1 and Fig 4-3. The small deviation is likely due to the 
vigorous qualify control measures applied to the GEAR datasets as well as the block averaging of the interpolated values.

Table 4-1: Statistical comparison of precipitation of observed and CEH-GEAR dataset at rain gauges for a period of 1995-2003 for Dee River

\begin{tabular}{ccccc}
\hline Station No. & Station Name & $\mathbf{R}^{2}$ & $\begin{array}{c}\text { RMSE } \\
(\mathbf{m m})\end{array}$ & NSE \\
\hline 1 & Hawarden Bridge & 0.98 & 0.51 & 0.98 \\
2 & Colomendy Centre & 1.00 & 0.38 & 1.00 \\
3 & Bala Lake & 1.00 & 0.59 & 0.99 \\
4 & Llangerwyn: Tan-Y-Llwyn & 0.99 & 0.52 & 0.99 \\
5 & Llanuwchllyn & 1.00 & 0.23 & 1.00 \\
6 & Tryweryn Dam No 2 & 1.00 & 0.42 & 1.00 \\
7 & Vivod & 1.00 & 0.16 & 1.00 \\
8 & Cefn Mawr & 0.99 & 0.63 & 0.98 \\
9 & Chester W WKS & 0.99 & 0.47 & 0.99 \\
10 & Eddisbury Fruit farm & 0.99 & 0.45 & 0.99 \\
11 & Mouldsworth P STA & 0.99 & 0.30 & 0.99 \\
12 & Tiresford & 0.79 & 0.51 & 0.98 \\
13 & Alwen Reservoir & 0.99 & 0.49 & 0.99
\end{tabular}

It is more useful to examine how different the areal rainfall generated area using both the gauge data and the GEAR data using the three pre-processing methods above. When it comes to the settings of SWAT, the Dee river catchments are delineated into 57 sub-basins. The 6-month moving averages of the areal rainfall over the selected sub-basins in the Dee catchments are shown in Fig. 4-4. The time series of GAM and GPM are very close (nearly identical) to each other for all six selected sub-basins.

The CPEM time series, however, is entirely different from the other two for most subbasins. Since the CPEM and the GPM method both use the value at the centroid of the sub-basins to represent the areal rainfall, such comparison in Fig. 4-3 indicates that the CPEM (which borrows the nearby gauge value) method may cause large deviation to the representation. It also shows that the spatial variation is not a big issue at such 
smaller sub-basins scale as the GAM and the GPM methods produce very close results.
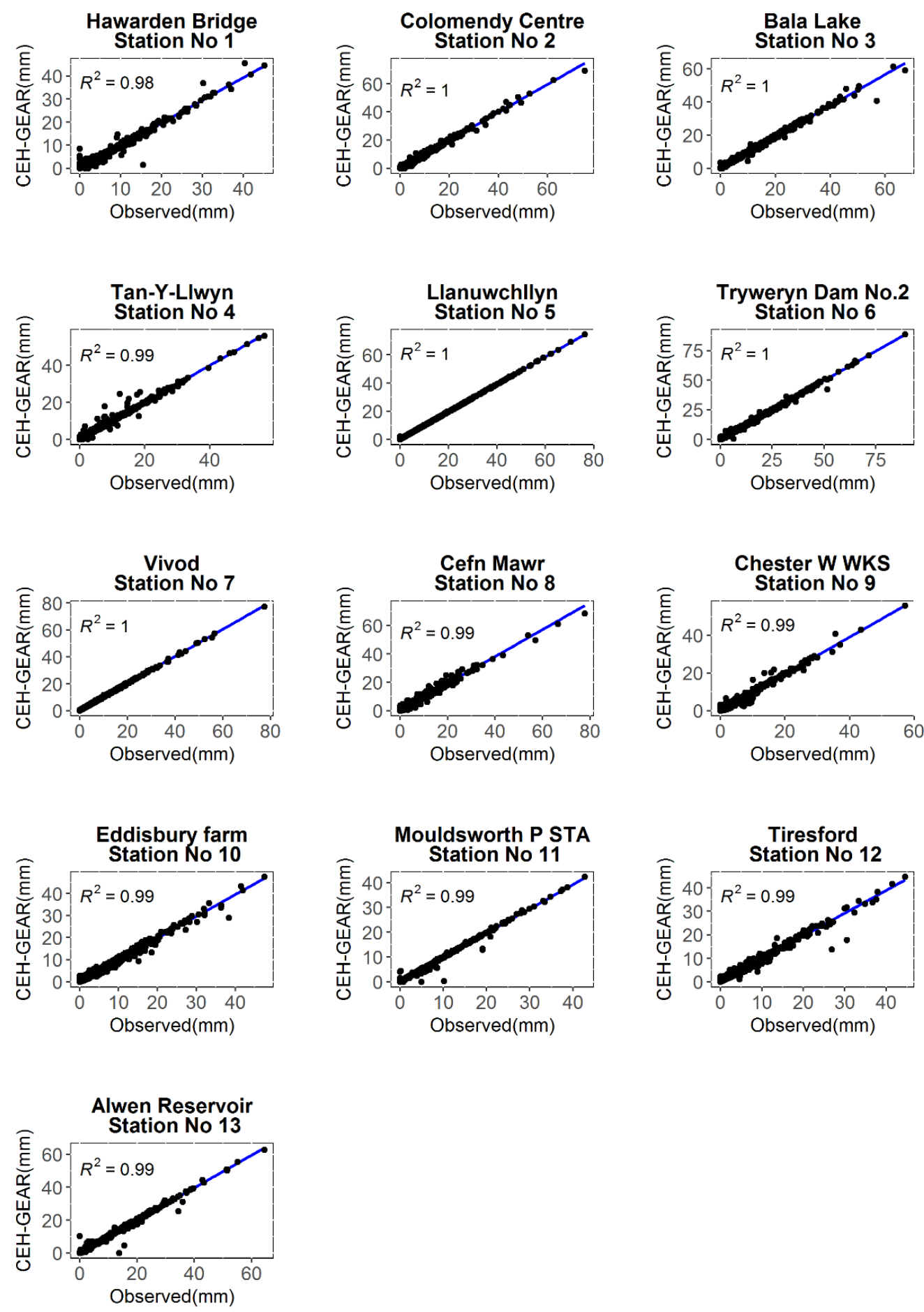

Fig. 4-3 Comparison of the daily precipitation observed by the gauges in Dee River catchments with the CEH-GEAR data at the same locations for the period of 1995- 

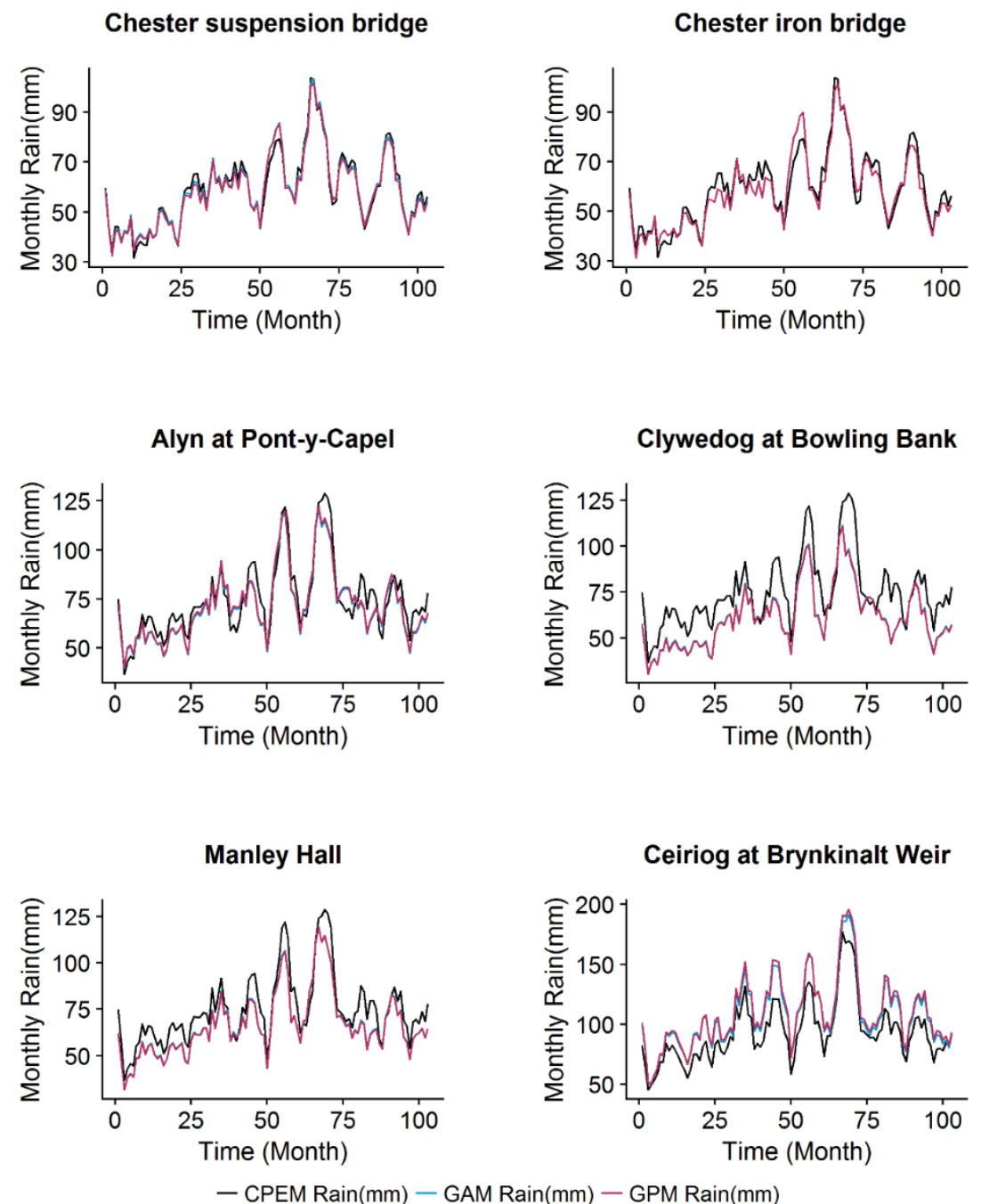

Fig. 4-4 Six-month moving average of monthly areal rainfall over the selected subbasins of the Dee river basin for the period of 1995-2003

The cross-sub-basin distributions give contrasting pictures as seen in Fig. 4-5. The CPEM methods produce a less varying distribution as some of the sub-basins share the same gauge. The GAM and GPM methods are able to reveal more details. As to the range of the annual averages shown in Fig. 4-5, the one from CPEM shows a range of 676-1,324 mm/year for CPEM and GAM 665-1,749 mm/year 663-1,692 $\mathrm{mm} /$ year for GPM respectively. 


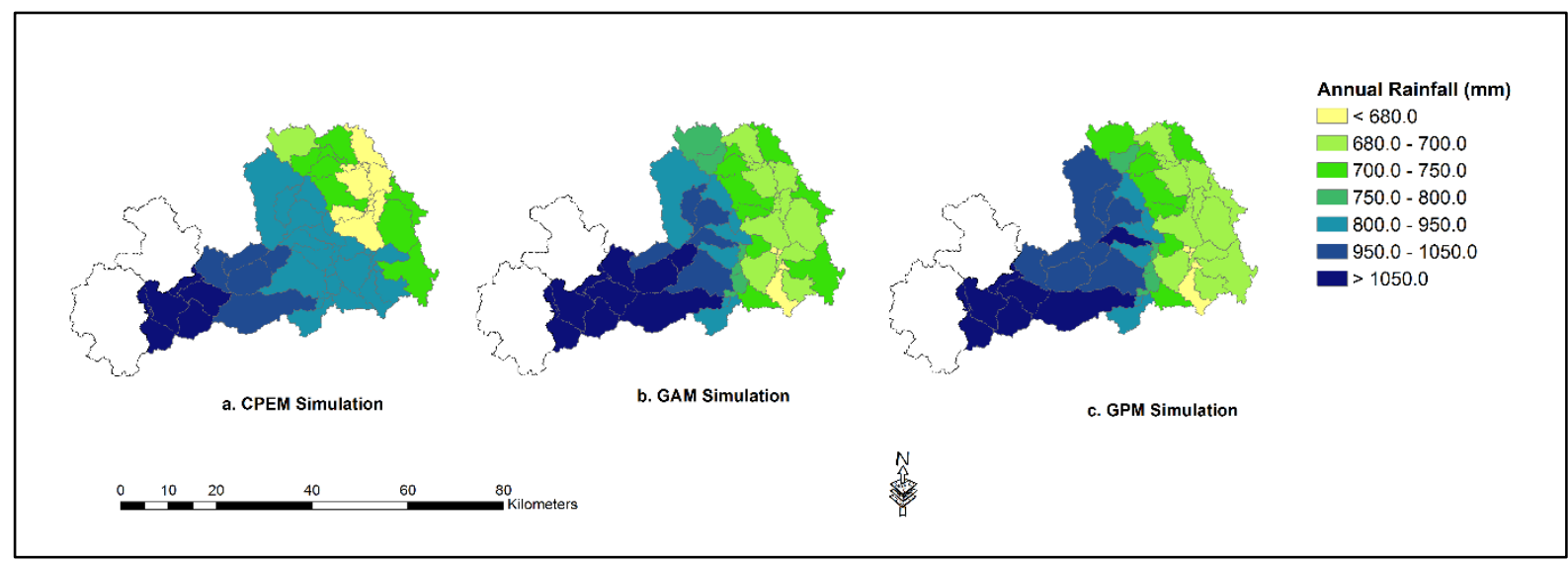

Fig. 4-5 Spatial distribution of annual rainfall for the three simulations at Dee river basin

The relative change maps of sub-basins rainfall of GAM and GPM with respect to default SWAT setup CPEM are illustrated in Fig. 4-6 with range of (-22.9 to 32) \% for GAM and (-23.2 to 27.7) \% for GPM.

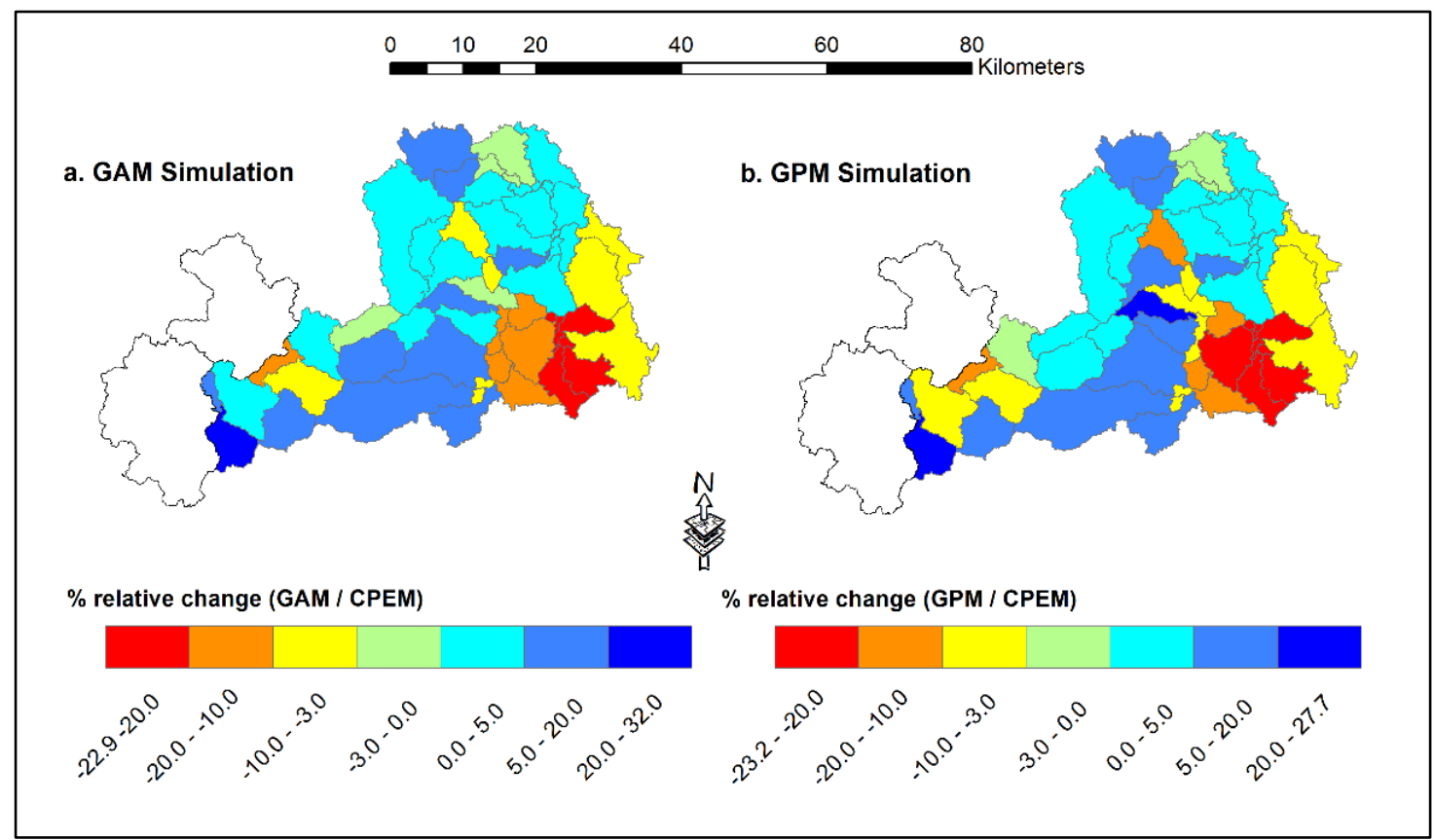

Fig. 4-6 Per cent of rainfall relative change of sub-basins rainfall for a. GAM against CPEM and b. GPM against CPEM [positive values refer to an overestimation rainfall of GAM/GPM over traditional SWAT setup, CPEM] 
It can be noticeably distinguished that in the south-eastern region of the Dee River basin the average annual rainfall of CPEM simulation is overestimated the values from GAM and GPM simulations. This is attributable to the fact there is a low density of rain gauges around this area (Fig. 3-1, page 34) and the nearest rain gauges to the centroid estimation fail to consider a satisfactorily spatial distribution of the precipitation. On the other hand, the region with complex terrain such as the south region of the mid-stream of the catchment has underestimation rainfall values of CPEM compared to GAM and GPM simulations. The likely reason is similar to the area overestimated rainfall, in addition to orographic effects.

\subsubsection{Impacts of rainfall pre-processing on model simulations}

To measure the effects of precipitation pre-processing on model calibrations, three SWAT models are calibrated for the Dee catchment using the three pre-processing methods CPEM, GAM and GPM respectively. Six river gauge stations are chosen to test the performance of the three calibrations by comparing the observed flow and the model simulated one. Further, one of the six stations, Brynkinalt Weir, is singled out to test the bias of the simulation. The performance of the three calibrated SWAT models for the Dee catchment is shown in Table 4-2.

Table 4-2: Calibration results of three simulations of the daily SWAT model for Dee river basin for the period of $1995-2000$

\begin{tabular}{ccccccc}
\hline \multirow{2}{*}{ Station } & \multicolumn{2}{c}{ CPEM } & \multicolumn{2}{c}{ GAM } & \multicolumn{2}{c}{ GPM } \\
\cline { 2 - 7 } & NSE & $\mathbf{R}^{2}$ & NSE & $\mathbf{R}^{2}$ & NSE & $\mathbf{R}^{2}$ \\
\hline Manley Hall & 0.93 & 0.94 & 0.94 & 0.98 & 0.94 & 0.98 \\
Iron bridge & 0.82 & 0.82 & 0.82 & 0.82 & 0.82 & 0.83 \\
Suspension bridge & 0.78 & 0.80 & 0.78 & 0.80 & 0.79 & 0.80 \\
Pont-y-Capel & 0.74 & 0.75 & 0.80 & 0.82 & 0.78 & 0.82 \\
Bowling Bank & 0.62 & 0.63 & 0.70 & 0.71 & 0.68 & 0.71 \\
Brynkinalt Weir & 0.54 & 0.65 & 0.66 & 0.70 & 0.65 & 0.69
\end{tabular}

Both the calibrations are driven by the GAM and GPM datasets outperform the one using the CPEM dataset (the original setting of SWAT). The improvements are not 
significant in the sub-basins where the CPEM driving model already does well, but they are more remarkable in sub-basins where it does not, e.g., the Bowling bank and the Brynkinalt Weir stations. Regarding the bias, a significant improvement can be seen for the Brynkinalt Weir sub-basins (Table 4-3).

Table 4-3: The per cent of bias (PBIAS) indices of the SWAT model calibrations at the Brynkinalt Weir station

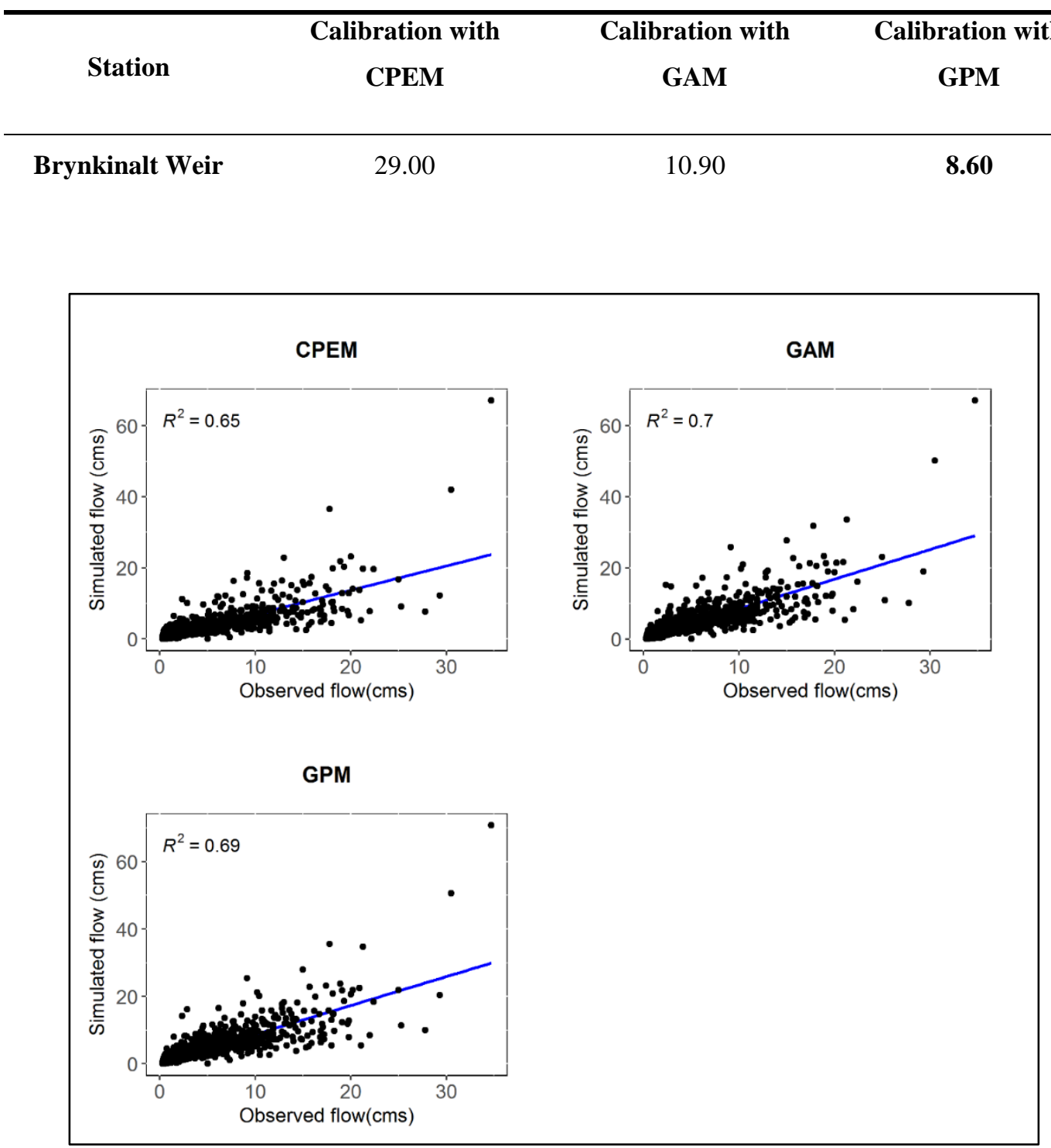

Fig. 4-7 Scatter plots of the simulated and observed flow from the three methods CEPM (a), GAM (b) and GPM (c) respectively at Brynkinalt Weir station for the calibration period 
For the overall fit, Fig. 4-7 picks up the calibration period as an example showing the performances of the three models calibrated at Brynkinalt Weir station. It is also evident that both the GPM- and GAM-driven calibrations are relatively better over the CPEM one with better simulations.

The PBIAS index is further examined in Table 4-4 which includes all nine combinations of cross-validation results. Interestingly, the validations using the GPM rainfall series give better results regardless of however the models were calibrated. For the other two indices NSE and $\mathrm{R}^{2}$, out of the nine combinations of calibrationvalidation with respect to the three different rainfall pre-processing methods (CPEM, GAM and GPM), GAM-GAM, GAM-GPM, GPM-GAM can achieve a better result as shown in Fig. 4-8 and 4-9. It is, however, surprised to see that the highest NSE at Pont-y-Capel station is from CPEM-CPEM. This possible reason is that the sub-basin connected to this station is small.

Table 4-4: Percent of bias of three simulations of the daily SWAT model for Brynkinalt Weir station of Dee river basin for the period of 2001-2003

\begin{tabular}{lccc}
\hline Calibrated Models & $\begin{array}{c}\text { Validation using } \\
\text { CPEM }\end{array}$ & $\begin{array}{c}\text { Validation using } \\
\text { GAM }\end{array}$ & $\begin{array}{c}\text { Validation using } \\
\text { GPM }\end{array}$ \\
\hline CPEM-Calibrated & 23.50 & 7.90 & $\mathbf{3 . 9 1}$ \\
GAM-Calibrated & 24.20 & 8.70 & $\mathbf{4 . 8 3}$ \\
GPM-Calibrated & 24.10 & 8.70 & $\mathbf{4 . 8 0}$
\end{tabular}

From the perspective of practical use, it is more interesting to look at how models consistently calibrated and validated by the same dataset behave. In this respect, it can be seen that the CPEM-CPEM setting (the original SWAT settings) remains as the worst; the GPM-GPM combination is the best in the PBIAS measurements for the selected sub-catchment, and overall the GAM-GAM combination does well across all sub-catchments in the Dee river experiment. 


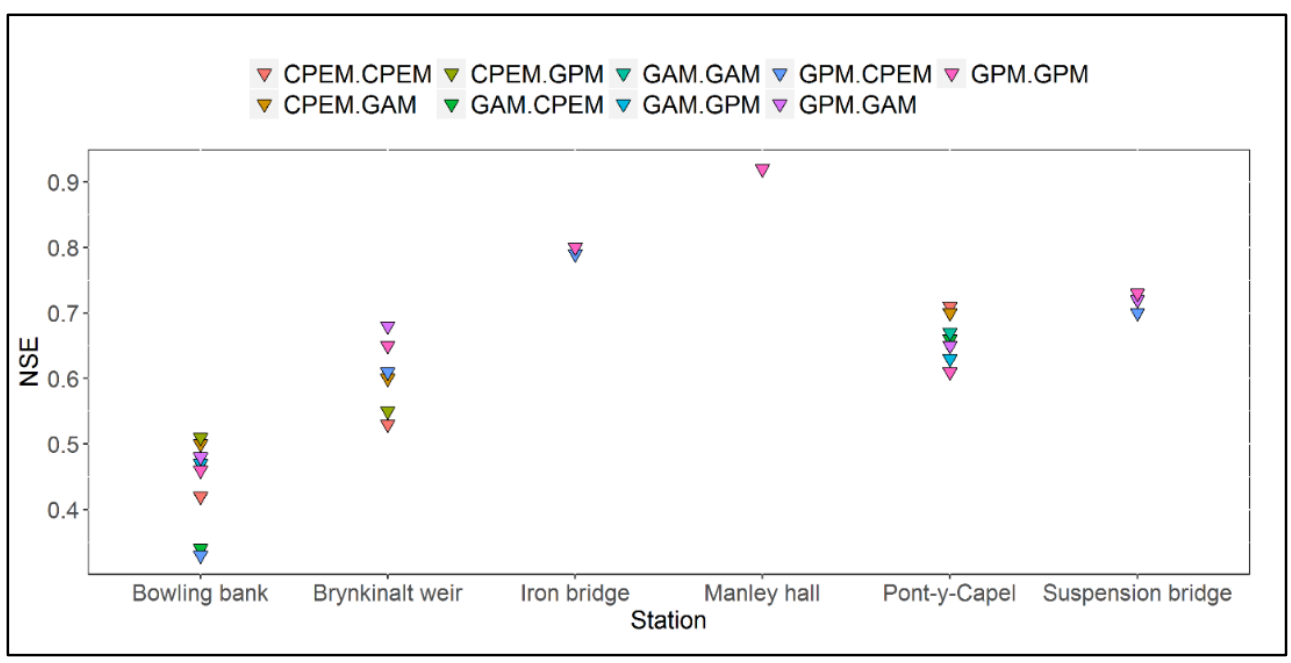

Fig. 4-8 Nash Sutcliffe coefficient of cross-validated results of three simulations of the daily SWAT model of Dee river basin for the period of 2001-2003

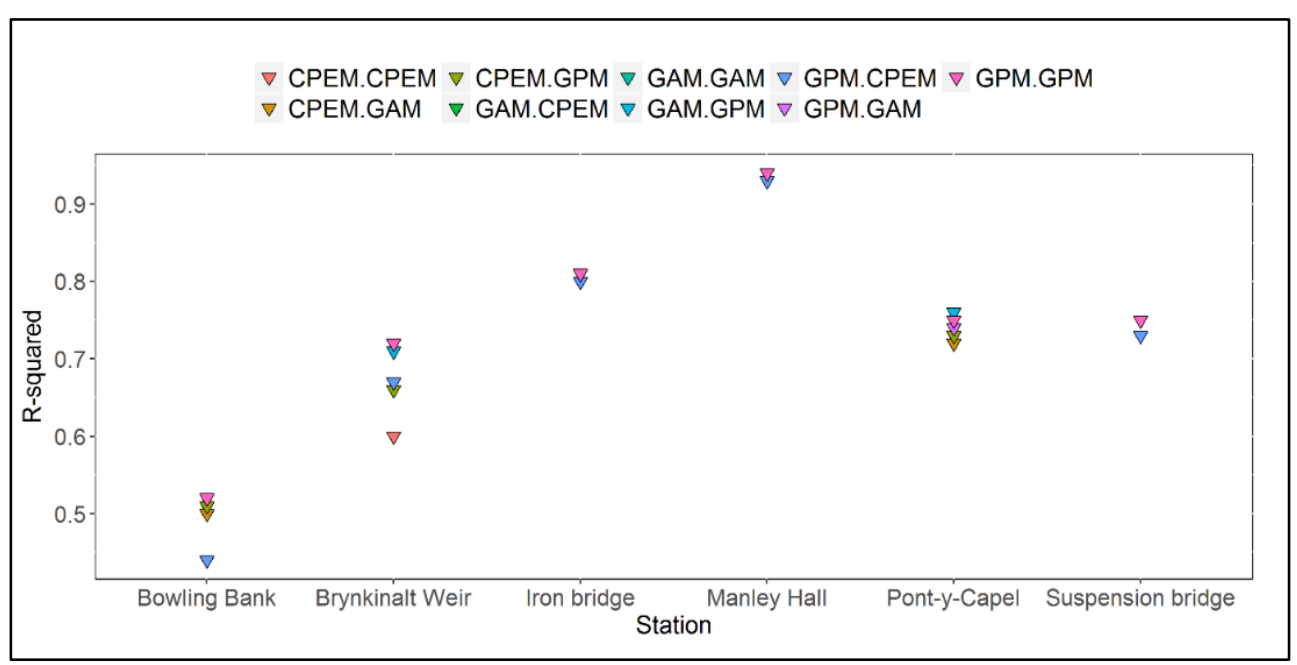

Fig. 4-9 Determination coefficient of cross-validated results of three simulations of the daily SWAT model of Dee river basin for the period of 2001-2003

The bias in model simulations can be related to the ill-parametrised model settings, but significant bias such as the one shown in Table 4-4 for the sub-catchment of Brynkinalt Weir is likely due to misrepresentation in rainfall inputs. Figure 4-10 shows the comparison of the simulated monthly river flows from the three SWAT models against the observed one at Brynkinalt Weir station for the entire period of 1995-2003. In general, all three simulations underestimate the river flow with the most considerable bias observed form the CPEM simulation; however, both the GAM 
and GPM driving simulations can recover and get much closer after the spinning-up period around the 36th month.

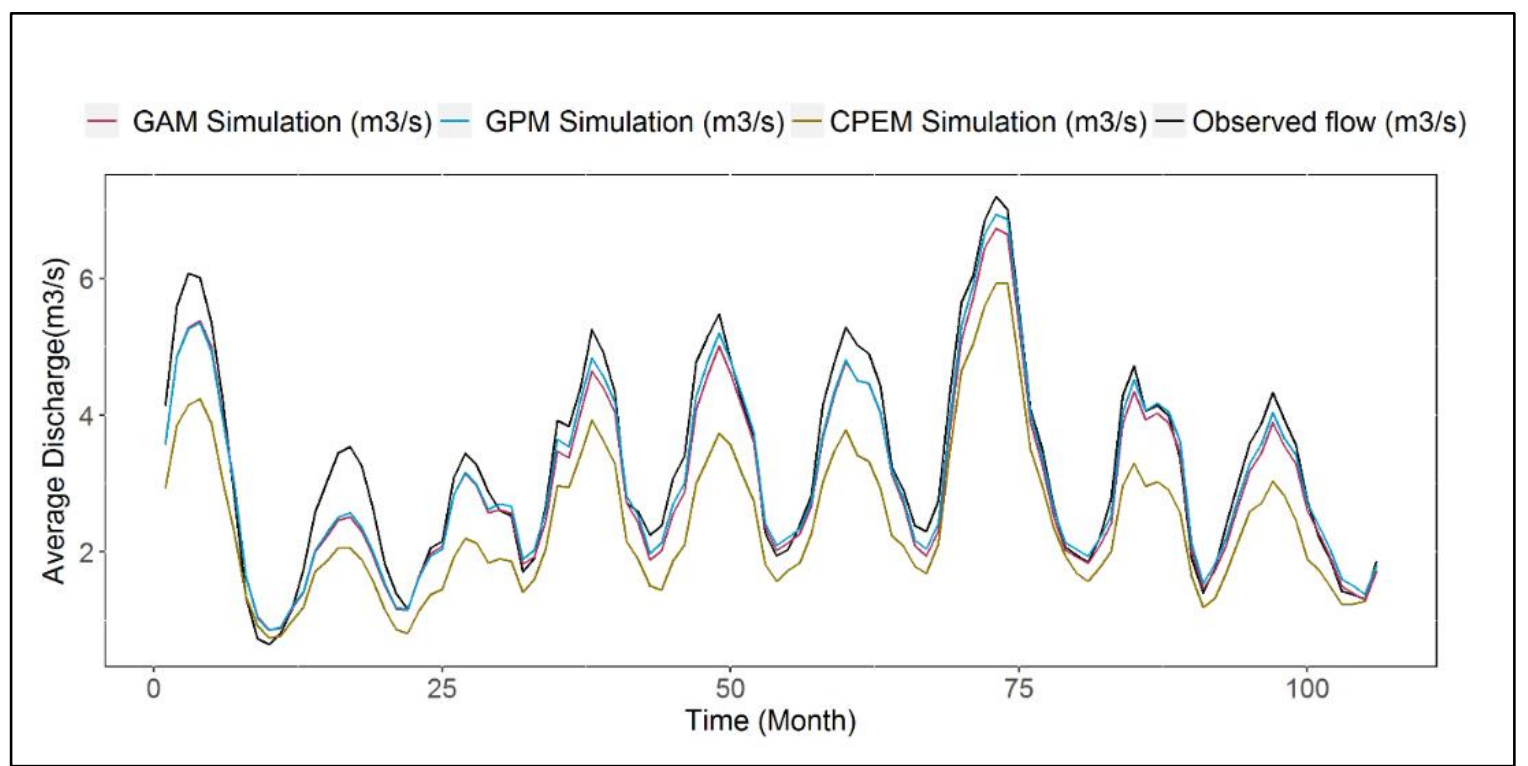

Fig. 4-10 The 6-month moving average of monthly river flow simulations at Brynkinalt Weir for the period of 1995-2003

A closer examination of the nine calibration-validation combinations over the validation period only (2001-2003) is revealed in Fig. 4-11. In this case, the cumulative simulated flows are compared against the observations. Several remarkable features are noticeably presented including:

1) Those models calibrated using GAM and GPM data produce nearly identical results in the cross-validation when using the same precipitation data;

2) Those driven by the GPM data in the validation perform best, irrespective of however they are calibrated; and those driven by GAM are in the $2^{\text {nd }}$ group next to the GPM driving one;

3) The CEPM data have worst yet very close performances regardless of how the models are calibrated; and

4) It is surprising to see that the model calibrated using the CPEM time series but validated utilising the GPM one achieves the best result, even though the difference from the other two (GAM-GPM and GPM-GPM) is tiny. 


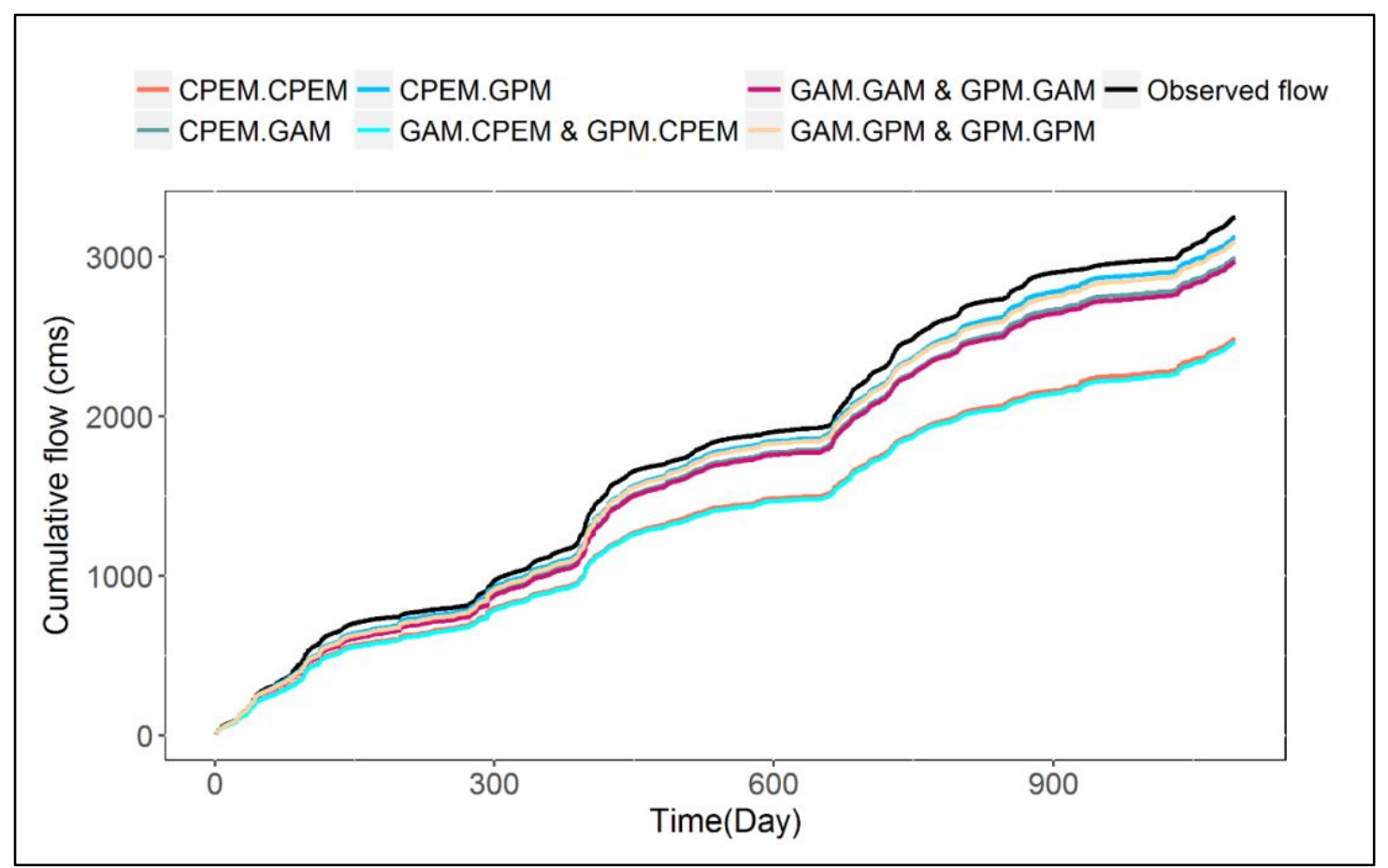

Fig. 4-11 Cumulative monthly flow simulations of Brynkinalt Weir station for the Period of 2001-2003

Fig. 4-11 effectively reconfirm what has been revealed in Fig. 4-7 and 4-10 which compares the overall performance of the nine simulations. In the case of the Dee catchment, it is shown that as far as the validation is concerned, the difference caused by various choices of models is small and hence 'stable' calibrations. However, the choice of feeding models with differently pre-processed rainfall inputs (datasets) does make significant improvements. In this particular case, the CEH-GEAR based GAM and GPM are a better choice than the rain gauge based CEPM method.

\subsection{Summary}

In this chapter, it is investigated how various areal rainfall pre-processing methods could impact on hydrological model performance. Thanks to the latest high-resolution and high-quality, gridded rainfall dataset, it is possible to measure such impact on calibration and validation of a semi-distributed model SWAT. The accompanying socalled 'compensation' due to model parameterisation was also studied by comparing the three distinctive models calibrated with different rainfall pre-processing methods: the centroid point estimate method (CPEM), the grid area method (GAM) and the grid 
point method (GPM). The models were further cross-validated over a different period to isolate the changes in performances due to model calibration (parameterisation) and the input rainfall data from different pre-processing methods.

Several important points can be concluded:

1) The quality of the CEH-GEAR dataset and the GAM/GPM processing method. It has been shown the GEH-GEAR data is consistent with the gauge measurements and can work as a reliable source for model calibration and validation. Based on this dataset, both GAM and the GPM methods are not only theoretically better than the default CPEM used by SWAT, but they also help to improve model calibration and validation significantly;

2) Impact on model calibration: Both GAM and GPM can improve model calibration by a considerable amount of margin against the default setting. The improvements are not as significant in the smaller catchment where rainfall distribution representation is less dominating. A remarkable finding is that the difference among the models calibrated using the three distinctive methods is not as vast as previously expected. In fact, these models behave very closely when fed with the same rainfall time series during validation. The variation in calibrated model parameters is also small;

3) Impact on cross-validation and practical implication. Although model calibration differs when using differently pre-processed rainfall data, it is the rainfall input data that dominates the cross-validation performance instead of how a model is calibrated. A less well-calibrated model due to the use of an inferior pre-processing method such as CPEM can do equally well when fed with better-pre-processed data such as GAM or GPM during validation. An accompanying implication is that in practice, a model previously calibrated with low-quality rainfall data can still use high-quality rainfall inputs when they become available at later times without having to re-calibrating which is often limited by the length of data; and

4) Impact of catchment size. The improvements due to the new input data/new pre-processing method become less significant when the catchment size gets smaller. A further detailed investigation with more catchments studied is 
needed. However, this can be well explained by the less spatial variation of rainfall over smaller basins than larger ones.

It should note that this study is based on a semi-distributed model which still treats the rainfall inputs in a very much lumped way, at least at the sub-catchment scale. The interactions among the rainfall inputs, sub-catchment parameterisation and the whole catchment response indeed require further studies that hopefully can identify the 'sensitive' areas where more sophisticated rainfall measurements and pre-processing can help significantly. Nevertheless, this research shows the value of high-quality datasets such as the CEH-GEAR in hydrological modelling, and a practical approach to improving the SWAT simulation by adopting the pre-processing methods like GAM and GPM even with conventional rain gauge measurements as they are not dependent on the CEH-GEAR data. 


\section{Chapter 5: Trend Analysis of Regional Water Resources}

Another important aspect needs to be considered in relation to the IWRM concept is the historical trend of the hydrological variables for climate change impact studies. Conducting trend analysis of climatic variables is one of the necessary steps in many climate change impact studies in which the trend is often checked against aggregated variables. However, there is also a strong need to consider the trend of data in different regimes, for example, high flow versus low flow, or heavy precipitation versus prolonged dry period etc. For this matter, the quantile regression $(\mathrm{QR})$ based method is preferred as it can reveal the temporal dependencies of the variable in question, not only for the mean value but also for its quantiles. As such, tendencies illustrated by the QR will be immensely helpful in practice where different mitigation methods need to be considered for a different level of severities.

In this chapter, several quantile regression-based methods are employed to analyse the long-term trend of rainfall records in two climatically different regions: The Dee River catchment with daily rainfall data over 1970-2004 (refer to chapter 3) and the Beijing metropolitan area in China with monthly rainfall data from 1950 to 2012. Two quantiles are used to represent extreme heavy rainfall condition (0.98 quantile) and severe dry condition ( 0.02 quantile). The trends of these two quantiles are then estimated using the linear quantile regression before being spatially interpolated to demonstrate their spatial distribution (for Dee River only). The results show that the quantile regression can reveal the patterns of both extremely wet and dry conditions of the areas. The apparent difference between the trends at chosen quantiles manifests the utility of using QR in this context. This chapter also demonstrates the use of quantile regression $(\mathrm{QR})$ based method that to reveal long-term trends of river flow and catchment water yields in Dee watershed.

\subsection{Overview}

In recent decades, owing to global warming, the change of climate has had an increasing impact on water resources, agricultural activities and environment (Shi and 
$\mathrm{Xu}, 2008)$. The increased variation in the magnitude and frequency of precipitation and temperature are among the critical impacts of climate change (Dinpashoh et al., 2014). It is projected that by the 2050s annual average runoff will increase by 10-40 $\%$ at the high latitude and some wet tropical regions, on the other hand, a decrease of 10-30 \% in some dry areas at mid-latitudes and in the dry tropics (IPCC, 2007). Trend analysis of climatic variables such as precipitation, temperature and streamflow are more favourite choices for water resources planning and management; and as such it has been widely reported in many recent studies.

Trend analysis has also been a favourite utility and has been used to reveal patterns of changes in hydrological variables. Such exploration usually is carried out over either historical record like precipitation and temperature, to assess whether there are indeed significant trends and to identify the possible causes of those changes; or in conjunction with the use of projection-driven hydrological simulations to manifest future trend (Arnell, 2004; Schneider et al., 2013; Piao et al., 2007). The methods adopted by those studies range from simple linear regression based manifestation to non-parametric methods such as the Mann-Kendal Test (Mann, 1945; Kendall, 1975).

Many researchers prefer those sophisticated methods for studying historical trends to using simple indicators such as the percentage of decrease or increase of aggregated values, e.g., catchment rainfall. This is due to practical need as simulations of detailed year-by-year variation may not be as useful given the amount of uncertainties, and in fact, climate projections of various scenarios are mostly a result of what-if simulations. Nonetheless, such projection-driven hydrological simulation approach has found many applications in climate change impact studies, especially for largescale studies where human interactions (regarding river flow regulation and water resource management) are either be neglected or modelled qualitatively.

When it comes to water resources management at the catchment level, the approaches above become increasingly insufficient and challenging to meet the demand for more fine-granulated information to assess climate change impacts. The reason lies in twofolds: on the one hand, most of those catchments already have management practice and regulation in place, such as physical flow controls, irrigation, surface and 
groundwater abstractions, etc. Modelling catchment hydrology under such scenarios apparently is a challenging process, let alone representing the impact of climate change that is often intertwined with human activities. On the other hand, general trend analysis of aggregated values, for instance, annual mean flow is not as useful for catchment level water resources management that usually needs more specific information about extreme scenarios, such as floods and droughts.

There are various studies on the trend of extreme precipitation events in different region of the world such as (Donat et al., 2013; You et al., 2011; Fu et al., 2008; Zhai et al., 2005; Powell and Keim, 2015; Tomassini and Jacob, 2009; Griffiths et al., 2003; Santos, 2014), where trends of numerous rainfall indices are testified over the last few decades, such as annual total precipitation, average wet-day precipitation, maximum 1- and 5-day precipitation, and number of heavy precipitation days, etc.

Equally, extreme events due to the lack of precipitation, e.g., droughts, can also have a considerable impact on economic and the environment. Unlike extreme precipitation events whose effects are often readily perceived as severe flooding, the onset of droughts is dependent on the number of factors apart from the precipitation. It also takes a more extended period for the impact of droughts to be fully appreciated than that of heavy precipitation events. Even the definition of droughts is a complicated business (Wilhite and Glantz, 1985) such as meteorological droughts (concerning precipitation variation), hydrological droughts (concerning water supply) and agricultural droughts (regarding crops growth).

It is of no surprise that many indices have been developed to indicate the onset of droughts as well as to measure the severity of them. To name just a few, e.g., standardised precipitation index SPI (McKee et al., 1993; Ganguli and Reddy, 2014); Palmer drought severity index (Palmer, 1965; Li et al., 2015); Vegetation drought response index (Brown et al., 2008); multivariate standardized drought index (Hao and Aghakouchak, 2013); the surface water supply index (Shafer and Dezman, 1982); the drought severity index (Mu et al., 2013). 
Essential use of those drought indices is in studying the distribution and the trend of the occurrence of droughts both spatially and temporarily (Liu et al., 2008; Zhang et al., 2015; Piccarreta et al., 2004; Bayissa et al., 2015). Similar techniques that are employed for detecting precipitation trends are also used in this respect. As one of the many examples, the popular Mann-Kendall test was used by Zhang et al. (2015) to look into the trend of droughts as represented by the SPI time series.

It is worth noting that although multi-factor based drought indices may be able to describe drought events more accurately, those using precipitation data only (hence for indicating meteorological droughts) remain very useful, especially when studying future climate where other factors, such as vegetation are often unavailable or need to be further derived. As to the methods used for trend studies, the ordinary linear regression is among the first choice.

This method is often accompanied with non-parametric methods, such as MannKendall test (Mann, 1945; Kendall, 1975) for further confirming the statistical significance of the trends detected, e.g., Martinez et al. (2012); Paulo et al. (2012) and Song et al. (2014). In many cases, the trend indicated by the fitted regression line may not be statistically significant, its gradient is used nonetheless as a rough indicator for it can be quickly conducted.

One of the main drawbacks of this commonly used method is that the trend it manifests is often expressed as the mean of climatic variables conditionally on time. Whilst this can still be very useful in general, it fails to gain further necessary insights as to how events associated with more extreme values vary with time. For example, water managers would be more concerned with the trends of severe storms or extreme dry spells than those of the 'mean' conditions. To a certain degree, such a problem can be mitigated by stratifying the data into different categories. However, one has to realise that doing so will effectively reduce the sample size and hence sacrifices the information of data variability. 
The quantile regression (QR) method (Koenker and Bassett, 1978; Koenker, 2005), on the other hand, extends the ordinary linear regression to explain how the quantiles of response variables are conditioned on the input variables, which offers a new window through which different regimes of the response variables can be examined in details. The QR method has found its many applications in econometrics but more recently saw an increasing number of environmental studies including for example, hydrological uncertainty (Weerts et al., 2010), analysis of streamflow distributions (Luce and Holden, 2009) and hydro-meteorological analysis (Villarini et al., 2011), to name just a few.

There is a need for identifying trends of climatic variables in different quantity regimes as mitigation measures would be more effective with such refined information. Since quantiles are often a convenient measure of the data departing from its mean and hence loosely being associated with the rarity of those values, it becomes more appropriate as well using QR to reveal the trend of 'extreme' events as indicated by different quantiles. A more vigorous approach to linking $Q R$ with extreme value distribution can be referred to Cai and Reeve (2013).

In this chapter, an application of QR is revealed in identifying the rainfall trends in two drastically different climate regions: The Dee river catchment in the UK and the Beijing Metropolitan area in China. The focus is set on the trend of both extreme wet and dry conditions as they are of great value as far as flood risk management and water resources management are concerned. A higher quantile 0.98 to represent the extreme wet condition - where severe flooding may occur, and a lower quantile 0.02 for the extreme dry condition where prolonged droughts may be induced and used. Furthermore, the representing of the spatial distribution of such trends can also help produce a coherent, refined spatial structure for the use of flood risk and water management purposes.

A new method is also demonstrated that integrates hydrological modelling of highlymanaged river basin with the quantile regression $(\mathrm{QR})$ technique for analysing 
distributed trends of flow and water yields over the study area for both dry (potential droughts) and wet (flooding) conditions. The medium-sized, the Dee river (refer to chapter 3 ) is studied using river flow data over more than 30 years (1970-2003). The basin is firstly modelled using the Soil and Water Assessment Tool (SWAT) focusing on separation and representation of the flow regulation and water abstraction in the sense of restoring the basin to its 'natural' state.

The model is previously calibrated and validated against the observed data as in chapter three before the two scenarios (with and without regulations) are designed to simulate long-term simulations (1970-2003) of river flow and sub-catchment water yields under the conditions specified by both scenarios. Finally, two preferred quantiles 0.5 and 0.98 are chosen to represent dry (potential droughts) and wet (potential flooding) conditions in addition to the median ( 0.5 quantile) to represent average trend before a quantile regression analysis is conducted to find the time dependency of the three quantiles over the 32-year time window.

The study benefits from the facts that:

1) The resultant trends (as represented by the gradient of the regression lines) are spatially distributed at sub-catchment level;

2) The impact (hence contribution) of river flow regulating is explicitly represented; and

3) The method can be readily extended to study future climate change impact (though not included in this study) and it is also able to incorporate future changes to the regulating rules.

\subsection{Study regions}

As the primary target of this chapter is the investigation of the trend of hydrological variables close to the extremes (drought and flooding), two drastically different climate regions are selected:

1) The Dee catchment in the UK, which is subjected to more flooding events (Natural Resources Wales and Environmental Agency, 2014); and 
2) The metropolitan area of Beijing in northern China which is expected more drought events (Song et al. 2014).

The annual rainfall over the Dee catchment ranges from $650 \mathrm{~mm}$ in the downstream region to the east, e.g. Chester city, to 1,200 $\mathrm{mm}$ in the upstream in the west (British Atmospheric Data Centre BADC, 2015). Daily rainfall records from the 13 rain gauges over a period of 35 years (1970-2004) are used in this study (as in Fig. 3-1, page 34). An explicit dependency of rainfall on local topography is seen in Fig. 5-1, where precipitation in the western mountainous area (gauges 3, 5, 6 and 13) are top of the rank and the rainfall to the east tends to be more uniform with much lower amounts as well as variation. Dee River is subjected to Flooding events from time to time.

According to Environment Agency Wales (2010), Flooding events have happened at several locations throughout the River Dee basin, mostly from the main River Dee and its major tributaries, nonetheless also from smaller watercourses. Significant floods were observed in 1890, 1946, and 1964 and recently in 2001.

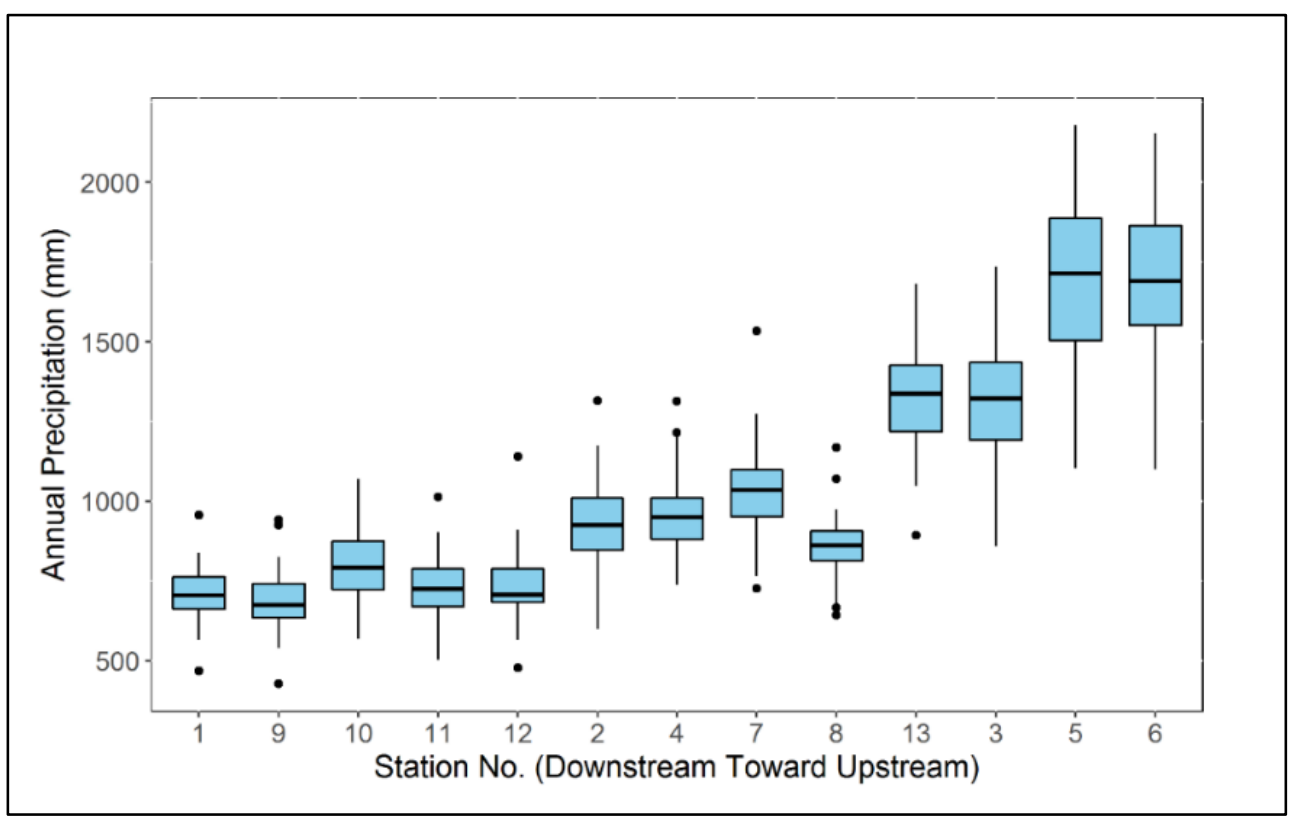

Fig. 5-1 Annual rainfall in and near Dee River catchment over the period of 19702004 
In contrast, the metropolitan area of Beijing is far more extensive $\left(16,410 \mathrm{~km}^{2}\right)$ yet with a similar layout of topography with its west and north $(68 \%$ in area) having elevation 1,000-1,500 m, while the central and south-east parts are just 20-60 m above sea level. The climate of this area is rather opposite to that of the Dee catchment, as it has a monsoon-driven humid continental climate which has cold and dry winters and hot humid summers.

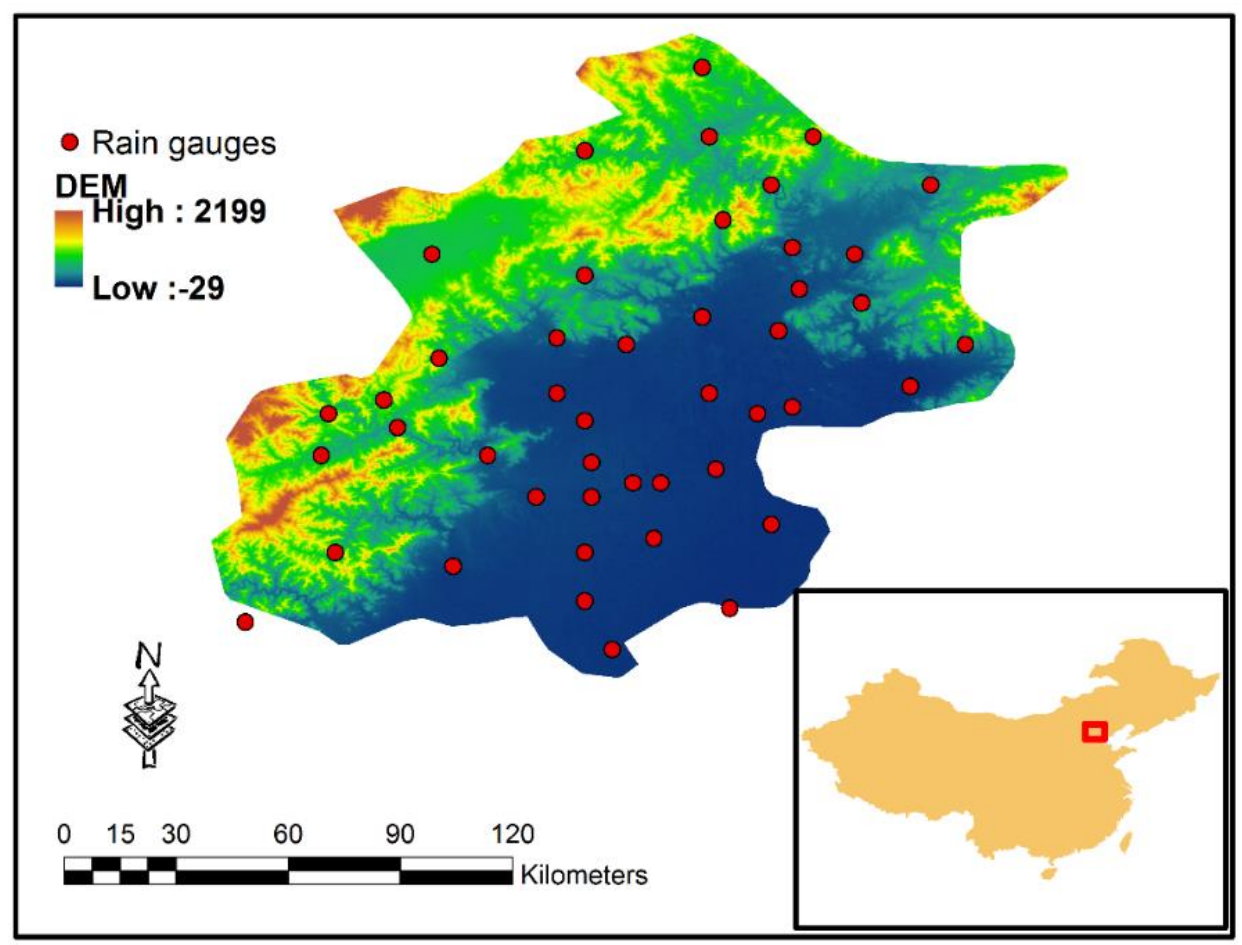

Fig. 5-2 Topographic map of Beijing with the location of rain gauges

Over the last two decades, the area has suffered both very dry winter/spring and yet wet summers which caused both severe shortages in the water supply as well as local flooding in the urban areas. Owing to limitations in data availability, only annual rainfall for 45 rain gauges over the entire region was obtained from 1960 to 2012. The areal rainfall is aggregated from the observation of a relatively dense rain gauge network as seen in Fig. 5-2. The variation of monthly rainfall over the region are shown in Fig. 5-3. 


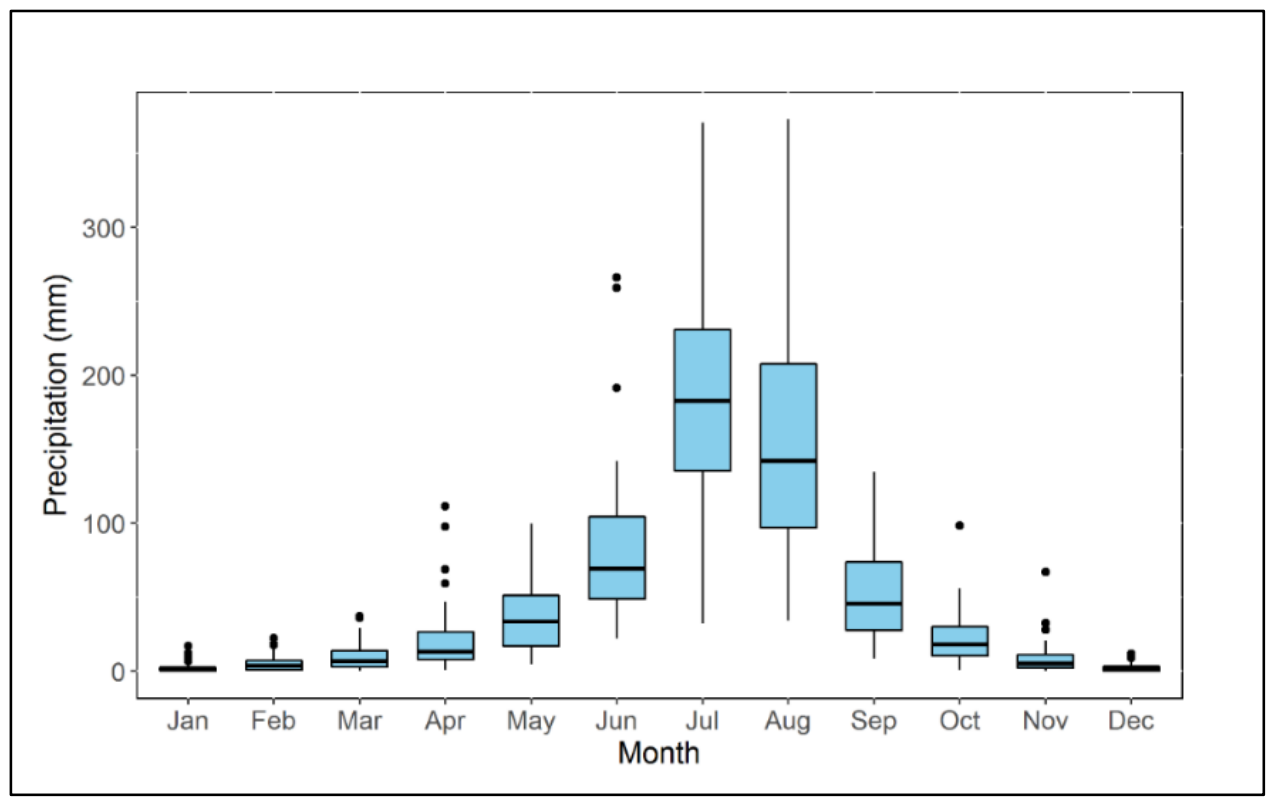

Fig. 5-3 Areal average rainfall in the metropolitan area of Beijing for each month over the period of 1960-2012

\subsection{Methodology}

\subsubsection{Quantile regression}

Quantile regression (Koenker and Bassett, 1978; Koenker, 2005) is a statistical technique that was initially introduced for conducting regression analysis in econometrics as an alternative and possibly better tool to the ordinary least square method (OLS). It has then been gradually applied in many other disciplines. The technique has received considerable attention in many statistical literatures but has less so in the areas related to water resources analysis (Tareghian and Rasmussen, 2013). In environmental studies, there have been several applications reported as illustrated in Table 5-1.

The QR method is a powerful extension to the ordinary linear regression in a sense that the quantiles of given response variables are conditioned on independent variables. As summarised by Koenker (2005), the QR method offers the following benefits:

1) The error terms are not necessary to be normally distributed; 
2) Does not assume homoscedastic variables (variables could be heteroscedastic);

3) It is not sensitive to outliers;

4) It can be used to predicts a desired quantile of the conditional distribution rather than mean; and

5) This approach is preferred when the interest is the analysis of distribution rather than average and when the core attention is the tail of distributions.

Table 5-1: Quantile regression example applications in environmental and climate change impact studies.

\begin{tabular}{ll}
\hline Application & Authors \\
\hline Analysis of streamflow distributions & Luce and Holden (2009) \\
Changes of annual rainfall in Zimbabwe over time & Mazvimavi (2010) \\
Estimation of predictive hydrological uncertainty & Weerts et al. (2010) \\
Hydro-meteorological analysis of a flood event & Villarini et al. (2011) \\
Statistical downscaling of precipitation & Tareghian and Rasmussen (2013); \\
& Cannon (2011); and \\
Analysis of Arctic and Antarctic sea ice extent & Friederichs and Hense (2007) \\
Quantile trends in Baltic sea level & Barbosa (2008) \\
Tropical cyclones trend changes & Elsner et al. (2008) \\
Trends in extreme precipitation indices & Fan and Chen (2016)
\end{tabular}

There are plenty of resources over the derivation of QR in the literature, e.g., Koenker and Bassett, (1978), Koenker (2005). A quick recap is presented here for illustration purposes. First, let considered $Y$ is a random response variable with cumulative distribution function $C D F F_{Y}(y)=P(Y \leq y)$. The $\tau^{\text {th }}$ quantile of $Y$ can be expressed as:

$$
Q_{\tau}(Y)=\inf \left\{y: F_{Y}(y) \geq \tau\right\}
$$


where $0<\tau<1$ is the quantile level and $Q_{\tau}(Y)$ is decreasing function of $\tau$ (i.e. $\mathrm{Q}_{\tau 1}(\mathrm{Y})<\mathrm{Q}_{\tau 2}(\mathrm{Y})$ for $\left.\tau_{1}<\tau_{2}\right)$. The linear conditional quantile function used in this study can be expressed as follow:

$$
Q_{\tau}(Y \mid x)=X^{\wedge} \tau \beta(\tau)
$$

where $x$ is the vector of dependent variable; $\beta(\tau)$ is the vector of parameters associated with the $\tau$ th quantile. Statistically speaking, each quantile of the conditional distribution in linear quantile regression is characterised by an individual hyper-plane and for individual set of observation (i.e. $X$ and $Y$ ) the parameters of can be estimated by:

$$
\hat{\beta}(\tau)=\arg \min _{\beta} \sum_{i=1}^{n} \rho_{\tau}\left\{y_{i}-x_{i}^{T} \beta\right\}
$$

Quantile regression is associated closely with models for the conditional median. The minimising the mean absolute error leads to an estimation of the conditional median of the predict and data. By applying asymmetric weights to positive/negative errors, for instance by using a tilted form of the absolute value function, one can instead compute conditional quantiles of the predictive distribution (Koenker and Bassett, 1978; Canon, 2011). Where $\rho_{\tau}$ is loss quantile function (also known as the check, tick, absolute value function or pinball loss function) (Cannon, 2011), it can be presented as follow:

$$
\rho_{\tau}(u)=\left\{\begin{array}{c}
u(\tau-1) \text { if } u<0 \\
u \tau \text { if } u \geq 0
\end{array}\right.
$$

where $0<\tau<1$. Given Predictors $\mathrm{x}_{\mathrm{i}}(\mathrm{t})(i=1 \ldots, I)$, slope $\mathrm{m}_{\mathrm{i}}$ and intercept $\mathrm{b}$ coefficients in a linear regression equation for the conditional $\tau$ - quantile $\hat{y}_{\tau}$ 


$$
\hat{y}_{\tau}(t)=\sum_{i=1}^{I} m_{i} x_{i}(t)+b
$$

can be calculated by minimising the quantile error function:

$$
E_{\tau}=\frac{1}{N} \sum_{t=1}^{N} \rho_{\tau}\left(y(t)-\hat{y}_{\tau}(t)\right)
$$

where $y(t)$ is the observed value of predictand at time $t(t=1,2,3 \ldots, N)$. The appropriate optimisation algorithms are outlined in Koenker (2005) and the outcome is the linear quantile regression model. Although the linear form of $\mathrm{QR}$ is most common, parametric models that are non-linear in parameters (i.e. models in which the model should explicitly specify the form of the non-linear regression equation) can also be estimated (Canon, 2011).

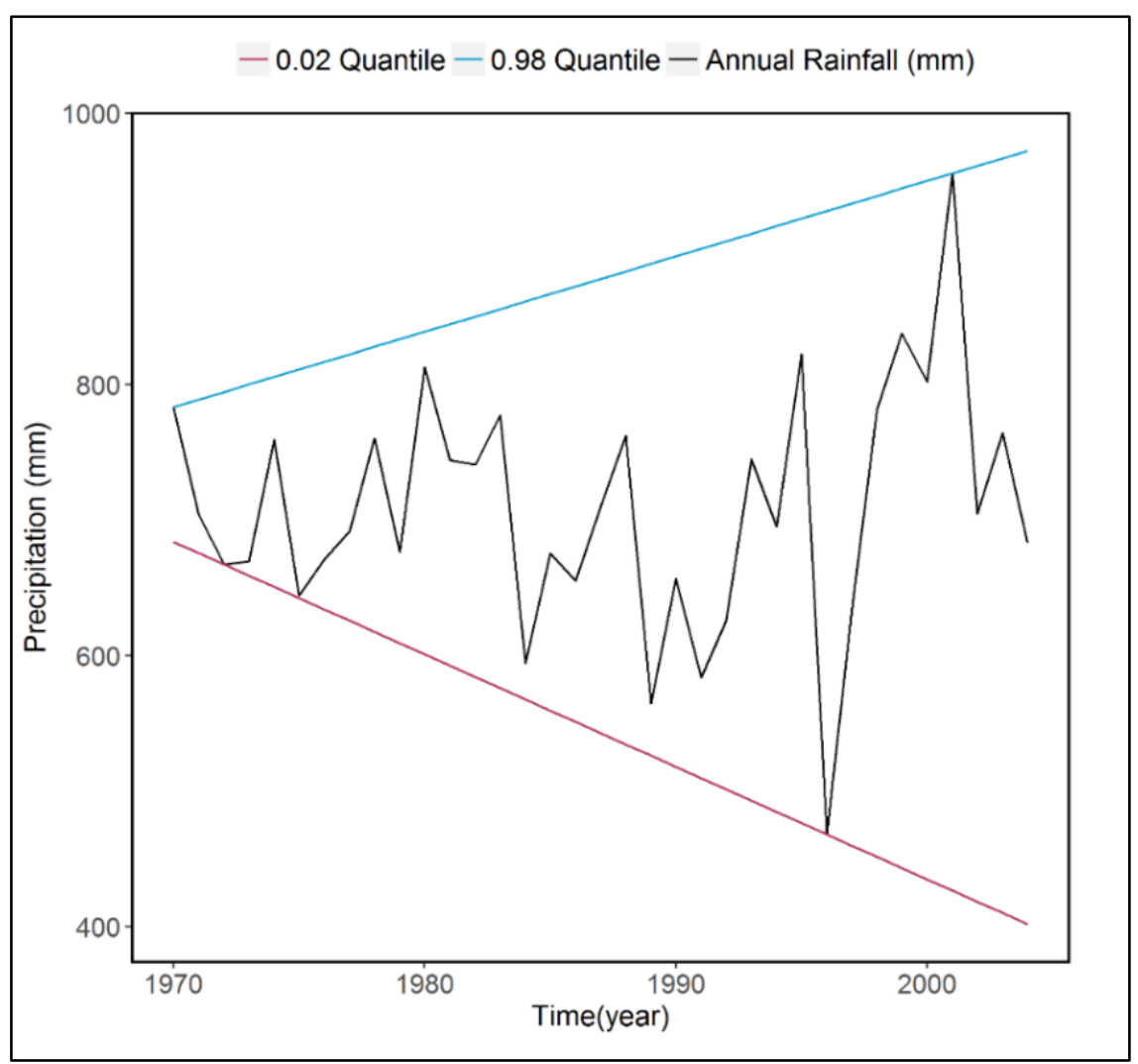

Fig. 5-4 Visual yearly linear trend for station No. 1 in Dee River basin for different quantiles 
The linear form of such a relationship can then be used to describe the magnitude (regarding its gradient or slope). Figure 5-4 shows an illustrative example of two regression lines representing the linear form of quantile-time relationship for the rainfall records taken at a rain gauge in the Dee catchment. It should be noted that by proposing the linear tendency of response variable quantiles on the input variable (time) it renders the process above parametric. The QR method is not limited to its linear form only; it can be easily extended to non-linear case.

In this study, a quadratic form to fit the rainfall trend associated with the flood (tau=0.98) and the drought $(\operatorname{tau}=0.02)$ is also adopted as in the following equation:

$$
Y=a+b t+c t^{2}
$$

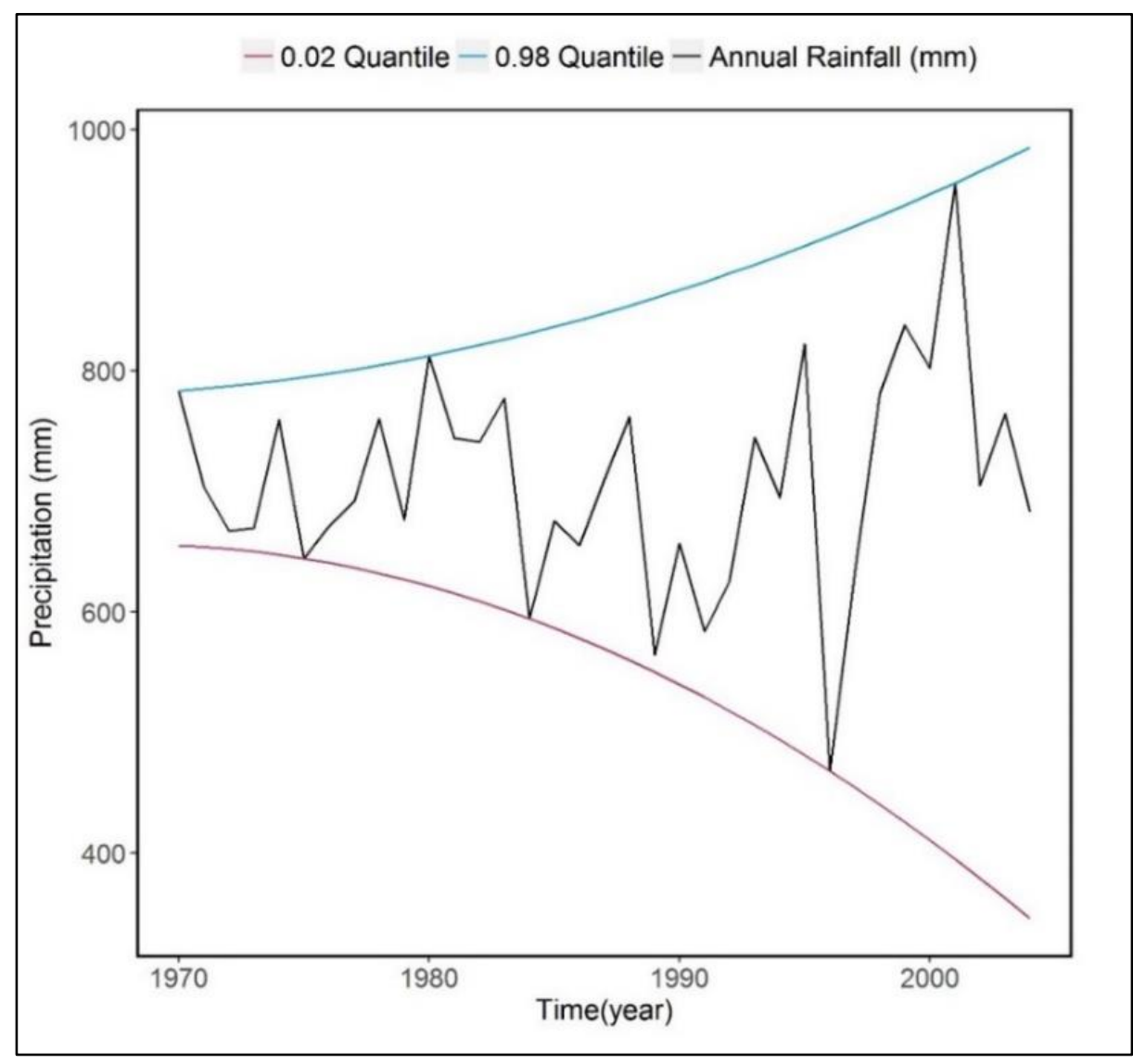

Fig. 5-5 Visual yearly quadratic trend for station NO. 1 in Dee River basin for different quantiles 
Figure 5-5 reveals an illustrative example of two regression lines representing the Non-linear form of quantile vs time relationship for the rainfall records taken at a rain gauge in the Dee catchment. Clearly, the nonlinear form of QR can obtain more detailed information as to the variation of the quantiles. While in this case, it would be impractical to specify a general trend as that can be derived from the linear counterpart, it can, however, identify how the pattern varies over time or sometimes even flips to other direction.

The choice of the linear form and the quadratic form implicates a parametric route. It should note that the QR method can also be nonparametric. However, to serve our purpose of identifying the general trend in the time series, it is convenient and beneficial to use linear quantile regression to have the first estimate of such a trend. Admittedly, non-parametric quantile regression would give a better fit in many cases and indeed needs to be explored in further studies.

\subsubsection{Significance test of trends}

A warranted question related to any trend analysis is whether the trend is statistically significant. For quantile regression, bootstrap methods are developed to test the significance of the fit. Discussion of this topic goes beyond the scope of this chapter. The analysis in this study is conducted using the R-package 'quantreg' (Koenker et al., 2016) which has integrated both the fitting methods as well as the significance test method.

\subsubsection{Choice of quantiles}

Another advantage of using QR in trend analysis is due to the natural link between the quantiles and the random events they represent. Indeed, this relationship is dependent on the underlying probability distribution. To overcome the unnecessary difficulties of fitting yet another distribution model, the widely used plotting method can be used 
to estimate such a link. One of the plotting position formulae is given by Gringorten (1963):

$$
p=\frac{r-0.44}{n+0.12}
$$

where $r$ is the rank of the data and $n$ is the sample size. By using this formula, it can be seen that the $0.98(0.02)$ quantile of the Dee rainfall records roughly repents an event of wet condition (dry condition) with a frequency of 1 in 64 years. Of course, such an estimate is not always accurate. Nonetheless, it indicates a 'mildly' extreme event.

\subsubsection{Standardised Precipitation Index (SPI)}

The QR method can be readily applied to the time series of precipitation directly. It would also be interesting to see how other measurements or indices derived from the rainfall vary with time. One of such indices is the Standardised Precipitation Index (SPI) developed by McKee et al. (1993) that has been widely utilised by the research community to indicate a range of conditions from extremely dry to extremely wet (Table 5-2) from rainfall observation. The SPI index measures how large the deviation is of a sample value from the mean of the population. The standardisation is achieved by dividing the difference by the standard deviation for a specific duration (McKee et al., 1993). For instance, a monthly rainfall value $x_{i}$, the corresponding SPI can be calculated as:

$$
S P I=\frac{x_{i}-\bar{x}_{i}}{\sigma}
$$

where $\sigma$ is the standard deviation of the population. The magnitude, length and duration of drought can be calculated with the SPI. Researchers have revealed that precipitation is follow the law of gamma distribution (Ganguli and Reddy, 2014; Zhang et al., 2015). The SPI can be computed for given periods $(3,6,9,12,24$ or 48 months). In our study, the monthly rainfall amount $x$ is fitted with a Gamma probability density function $g(x)$ as follows: 


$$
g(x)=\frac{1}{\beta^{\alpha} \Gamma(\alpha)} x^{\alpha-1} e^{\frac{x}{\beta}} \text { for } x>0
$$

Table 5-2: Classification of SPI values (McKee et al., 1993)

\begin{tabular}{cc}
\hline SPI Value & Drought Category \\
\hline$\geq 2$ & Extremely wet \\
$1.50-1.99$ & Very wet \\
$1.0-1.49$ & Moderately wet \\
$(-0.99)-0.99$ & Normal condition \\
$(-1.49)-(-1)$ & Moderately dry \\
$(-1.99)-(-1.5)$ & Very dry \\
$\leq-2$ & Extremely dry
\end{tabular}

where $x$ is the amount of rainfall and $\Gamma(\alpha)$ is the Gamma function. $\alpha$ and $\beta$ are the shape and scale parameters respectively:

$$
\begin{gathered}
\alpha=\frac{1}{4 A}\left(1+\sqrt{1+\frac{4 A}{3}}\right) \\
\beta=\frac{\bar{x}}{\alpha} \\
A=\ln (\bar{x})-\frac{\sum \ln (x)}{n}
\end{gathered}
$$

In these equations, $n$ is the number of precipitation observations. The cumulative probability distribution function is defined as follows:

$$
G(x)=\int_{0}^{x} g(x) d x=\frac{1}{\beta^{\alpha} \Gamma(\alpha)} \int_{0}^{x} x^{\alpha-1} e^{\frac{x}{\beta}} d x
$$

The Gamma function becomes undefined when $x=0$ and this situation does occur. In this case, the cumulative probability distribution is defined as follows:

$$
H(x)=q+(1-q) G(x)
$$


where $q$ epitomises the probability of zero value. If $m$ is used to denote the zero values in a precipitation series with $n$ observations, then $q=m / n$. In this study, an R package 'precintcon' (Povoa et al., 2016) is used to produce the annual SPI values at each rain gauges; then the $\mathrm{QR}$ method was applied to this series to investigate its variation over time.

\subsubsection{Extreme precipitation indices}

Higher quantiles, e.g., 0.98 of annual precipitation may be used to indicate a higher chance of flooding. There are, however, other indices associated with shorter duration may be more appropriate to describe possible flooding conditions. Following Donat et al. (2013) the following four indices were produced using daily precipitation data of the Dee catchment over every year:

1) Total precipitation on the very wet day (daily precipitation $>95^{\text {th }}$ percentile) R95PTOT;

2) Total precipitation on the extreme wet day (daily precipitation $>99^{\text {th }}$ percentile) R99PTOT;

3) Days with heavy precipitation (daily precipitation > $10 \mathrm{~mm}$ ) R10MM; and

4) Days with very heavy precipitation (daily precipitation $>20 \mathrm{~mm}$ ) R20MM.

\subsection{Results and discussion}

\subsubsection{Rainfall trend}

For the Dee catchment, daily rainfall records from the 13 rain gauges were aggregated into monthly and yearly datasets upon which the linear trend of 0.98 and 0.02 quantiles are produced at each rain gauges, before being interpolated over the catchment using Inverse Distance Weighted (IDW) method. As shown in Fig. 5-6, there is a basin-wide positive trend of 0.98 quantiles. There is also a clear spatial pattern associated with this overall positive trend with strong gradients ( $>20 \mathrm{~mm} / \mathrm{year})$ in the western coastal area gradually decreasing to the flat east area $(\sim 3 \mathrm{~mm} / \mathrm{year}$, Fig. 5-6a). Additionally, the trends at 10 out of 13 rain gauges are statistically significant. 
However, the 0.02 quantile trend is not so uniform, with an increasing trend to the west and southwest and a rather negative trend covering the rest part. It is also worth noting that for the low quantile, the trends shown at most gauges are not significant (Fig. 5-6b). However, those stronger negative trends are significant. In other words, the catchment is shown to be even wetter for the extreme conditions (especially in the west), but only the northeast part becomes dryer significantly for the extremely dry conditions.

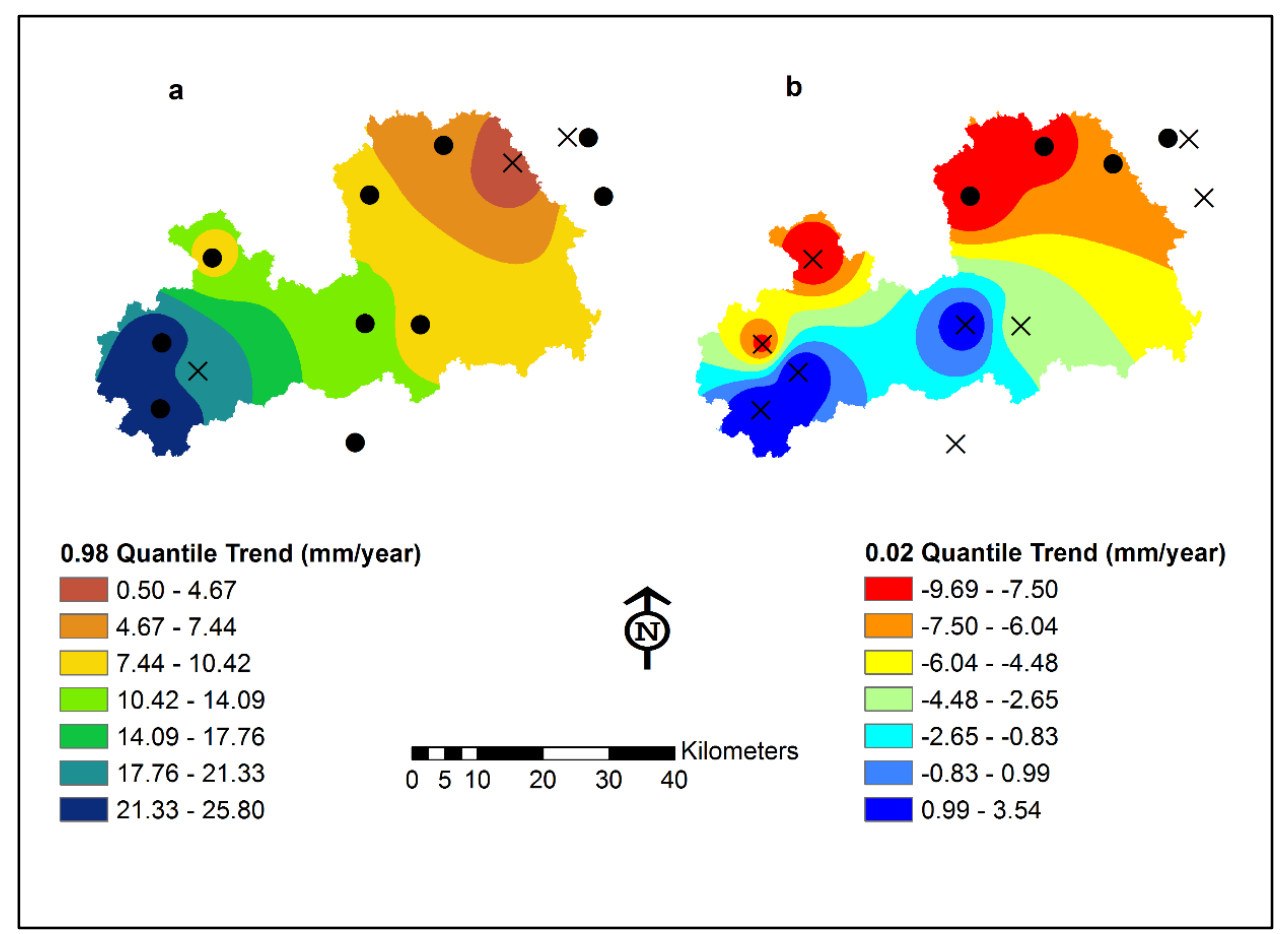

Fig. 5-6 Spatial distribution of the linear trend of annual precipitation over Dee River catchment (circular dots represent significant trend points)

Figures 5-7 and 5-8 demonstrate the results of QR trend analysis (linear Fig. 5-7 and non-linear in Fig. 5-8) of the annual precipitation at every gauge station. The linear QR analysis shows that most stations had an increasing trend for the upper quantile (0.98) and mixed (increase/decrease) trend at lower quantiles (Fig. 5-7). For the nonlinear form, it strives to capture more details of the variation. 
Station 1

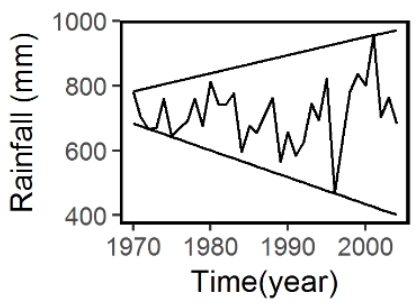

Station 4

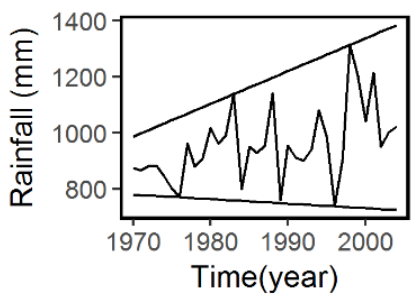

Station 7

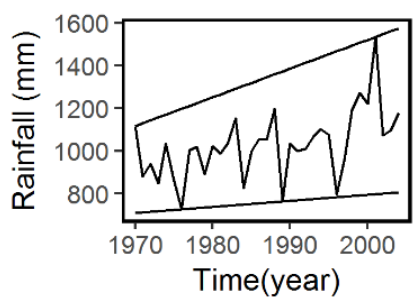

Station 10

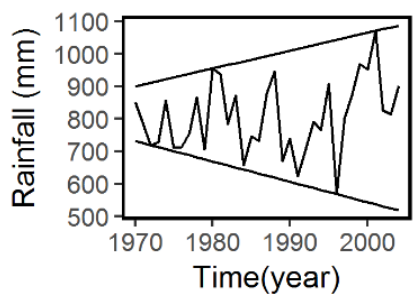

Station 13

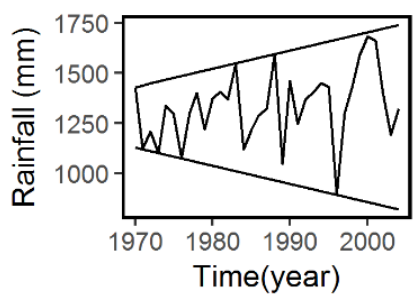

Station 2

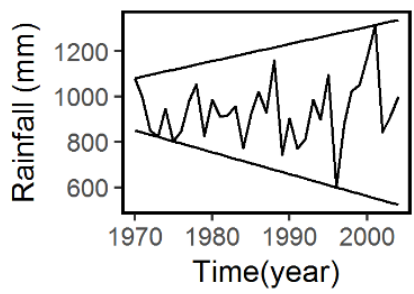

Station 5

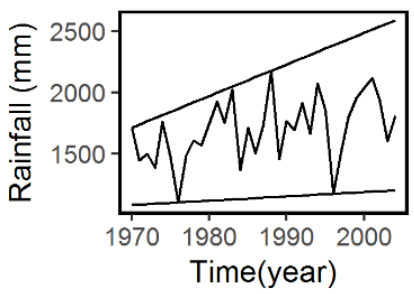

Station 8

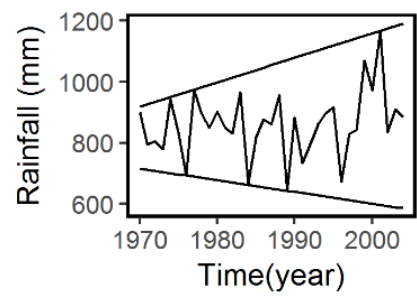

Station 11

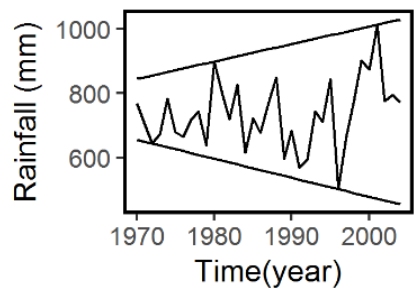

Station 3

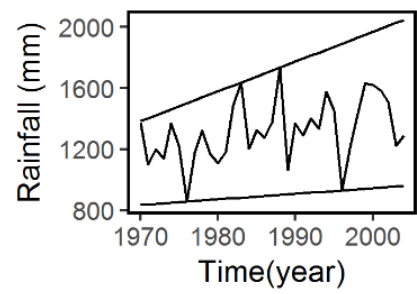

Station 6

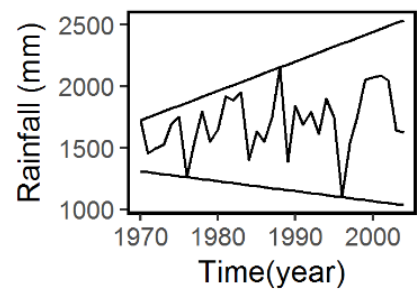

Station 9

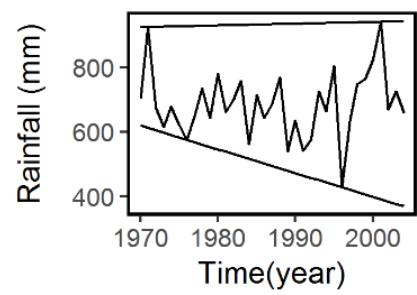

Station 12

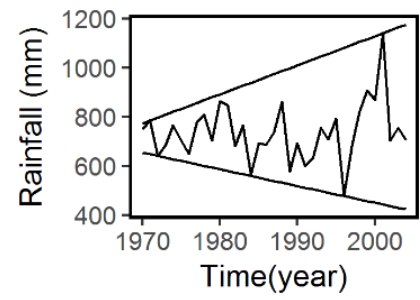

Fig. 5-7 Yearly linear trends using Quantile Regression for Flooding (tau=0.98) and Drought (tau=0.02) Conditions over Dee River Basin 
Station 1

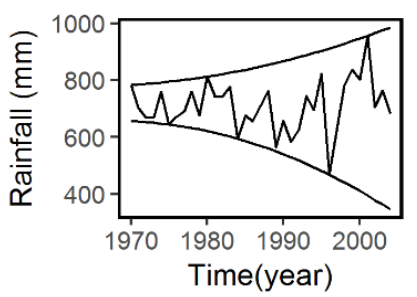

Station 4

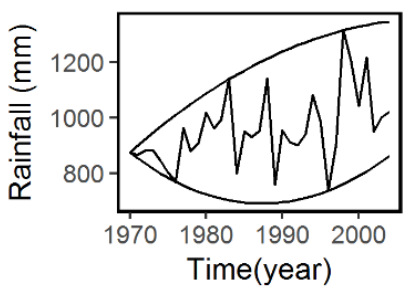

Station 7

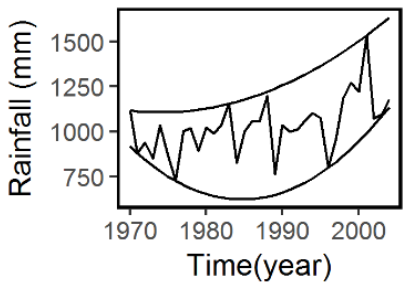

Station 10

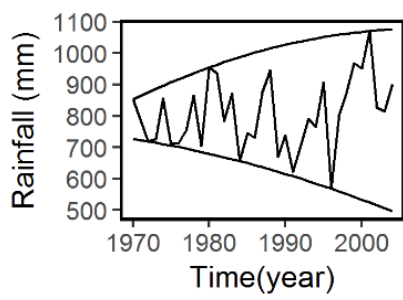

Station 13

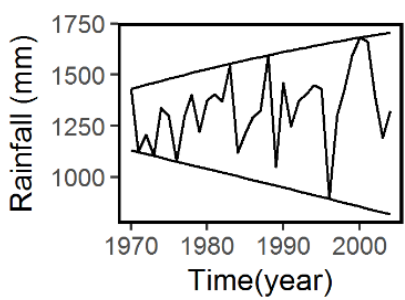

Station 2

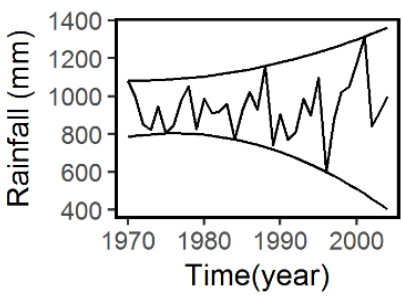

Station 5

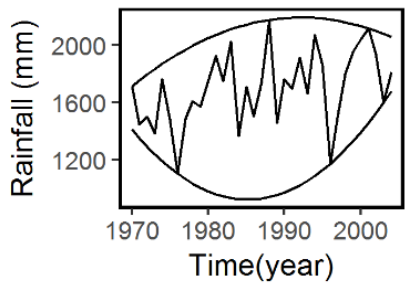

Station 8

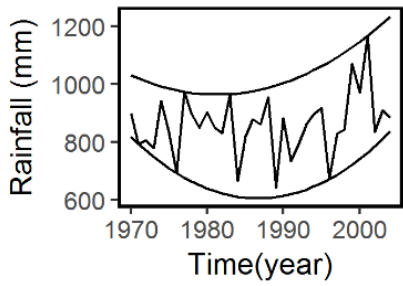

Station 11

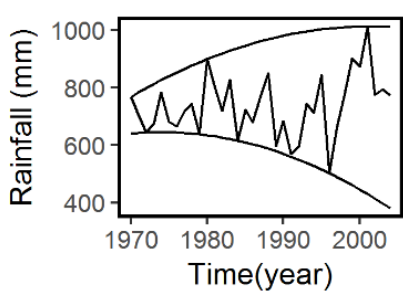

Station 3

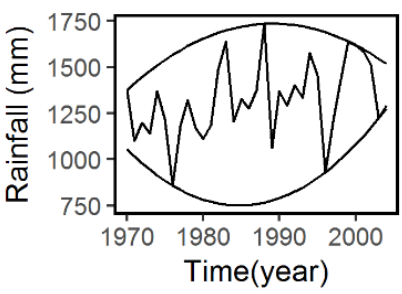

Station 6

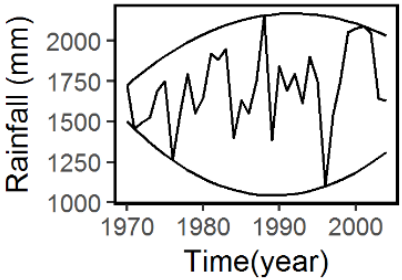

Station 9

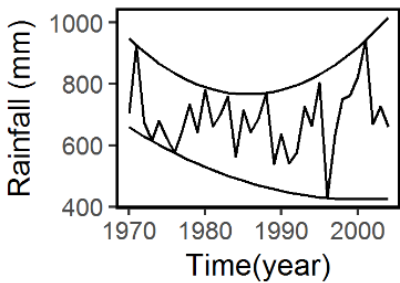

Station 12

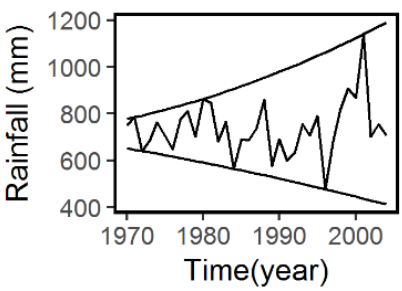

Fig. 5-8 Yearly quadratic trends using quantile regression for flooding (tau=0.98) and drought (tau=0.02) Conditions over Dee River Basin 
The difference between the two is well depicted in station 2 and station 8 , where the nonlinear fitting considers both the peak in the middle for the higher quantile and the increasing of the lower quantile at the end of the period. To some extent, the nonlinear QR can reveal the local peak and trough during the period hence a more comprehensive picture of the trends. Seemingly, more details can be revealed with a different nonlinear formation of $\mathrm{QR}$ instead of the quadratic one chosen there. It can even go further to adopt a nonparametric one as well. However, to avoid the potential trade-off of overfitting, it is believed that the linear form and the quadratic form used here are sufficient to describe the annual precipitation trend in this case.

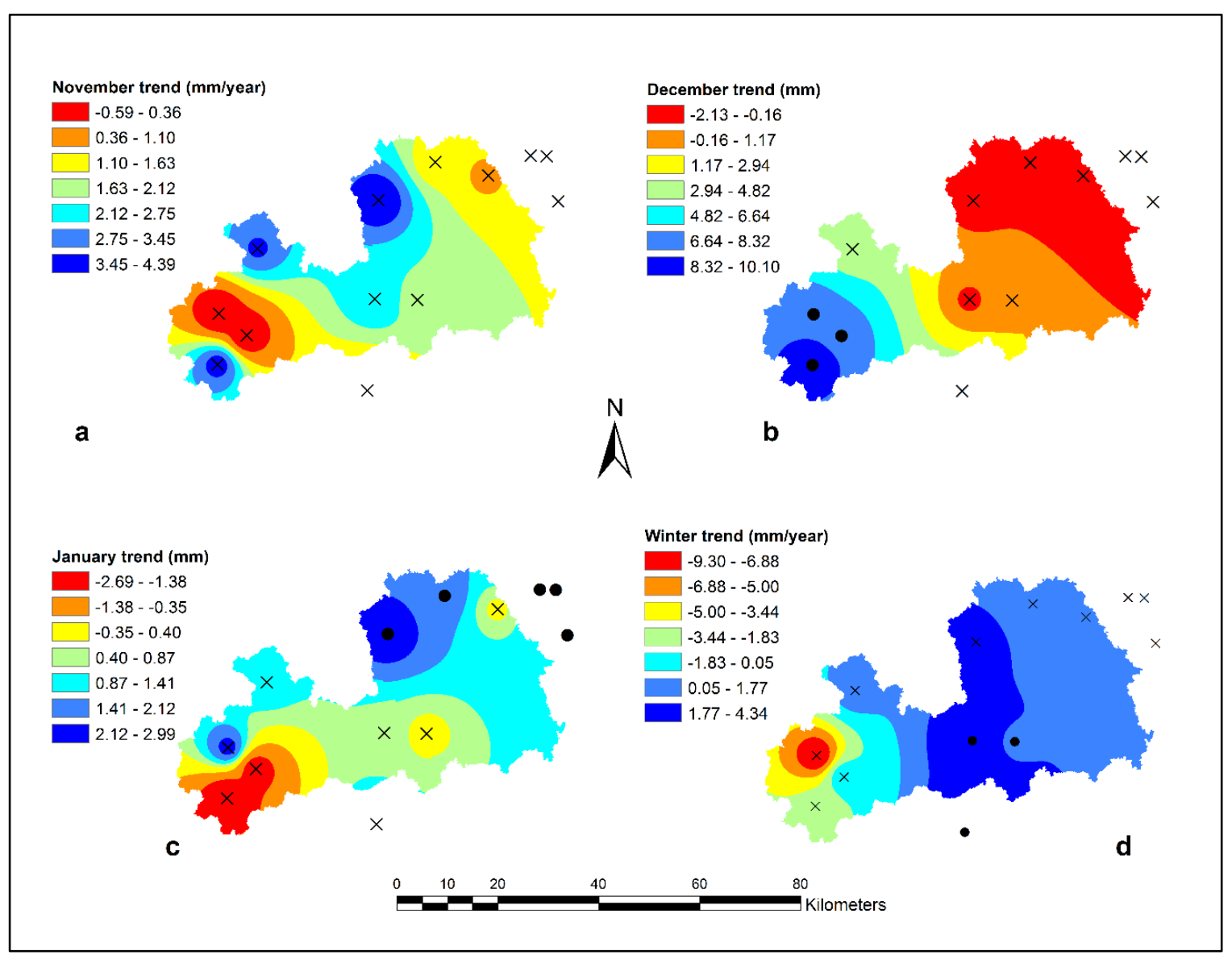

Fig. 5-9 Spatial distribution of monthly rainfall of winter months in the Dee catchment (a November, b December, c January and d November - January) for 0.98 quantile [circular dots represent significant trend] 
On the monthly time scale, the trend of winter months (November, December and January), together with the combination of these three months (winter trend) are shown in Fig. 5-9. The trends of November, January and winter (aggregation of November, December and January) appear to have a similar pattern with increasing trend in the mid and the downstream part of the River basin and decreasing trends in the upstream as shown in Fig. 5-9a, 5-9c and 5-9d. Interestingly, the December map depicts an opposite pattern as illustrated in Fig. 5-9b. It is remarkable that in general winter months do not have statistically significant trends at most gauges.

The spatial patterns of the trends related to the extreme precipitation indices are revealed in Fig 5-10 and Fig 5-11. For both R95PTOT and R99PTOT, a strong dependency on local topography can be seen (Fig 5-10) which indicates an increasing trend of both indices in the western mountainous area while the eastern part of the catchment shows a decreasing trend.

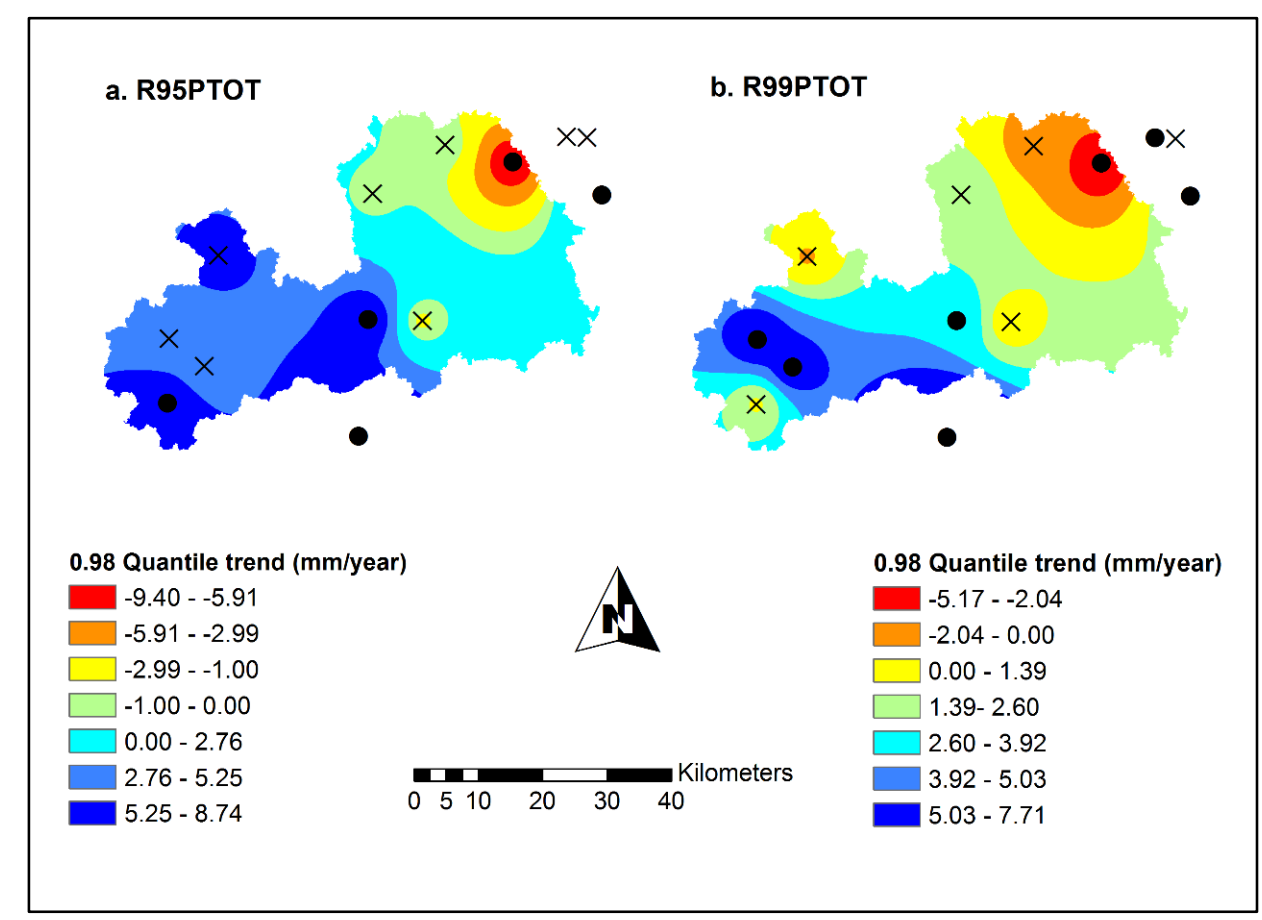

Fig. 5-10 Spatial distribution of the linear trend of annual precipitation of 0.98 quantile over Dee River catchment for R95PTOT and R99PTOT indices (circular dots represent significant trend points) 
In other words, areas receiving more precipitation are having more extreme events in comparison with those receiving less rainfall. For the other two indices R10MM and R20MM, Fig. 5-11 reveals a more random pattern with a large area of the catchment showing a minimal increasing trend for the number of days in a year having heavy rainfall.

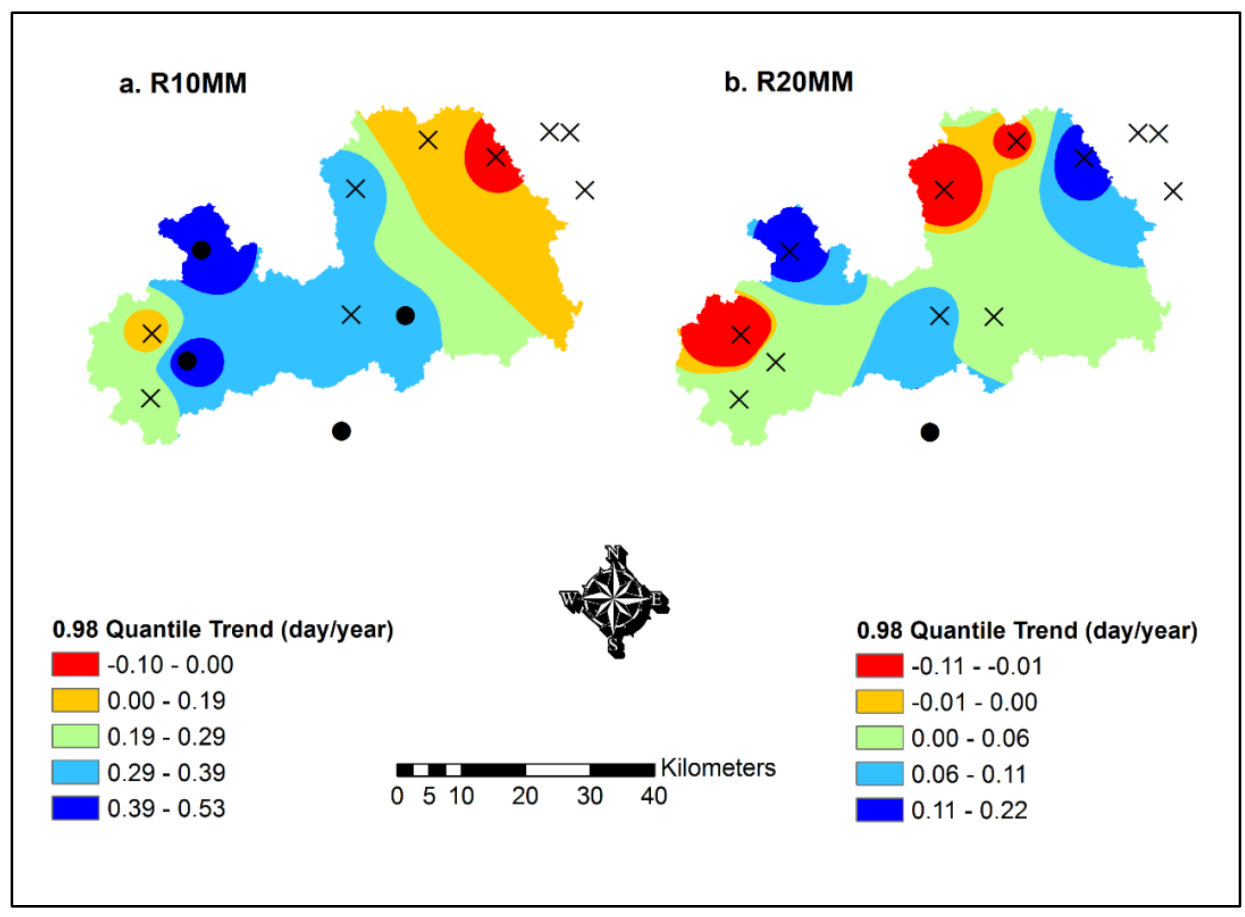

Fig. 5-11 Spatial distribution of the linear trend of annual precipitation of 0.98 quantile over Dee River catchment for R10MM and R20MM indices (circular dots represent significant trend points)

The SPI index can be used to indicate both wet and dry conditions. The upper quantile (0.98) associated with the wet condition and the lower (0.02) quantile always associates with the dry condition. Fig 5-12 shows an overall increasing trend of the 0.98 quantile of SPI and mostly decreasing trend of the lower quantile SPI except in the middle of the catchment. This can be interpreted as overall the wet years have become even wetter for most of the area and the dry years are getting drier except the middle part of the catchment. Again, such patterns are consistent with the overall trends of the precipitation itself, i.e., a widening gap between wet and dry years. 


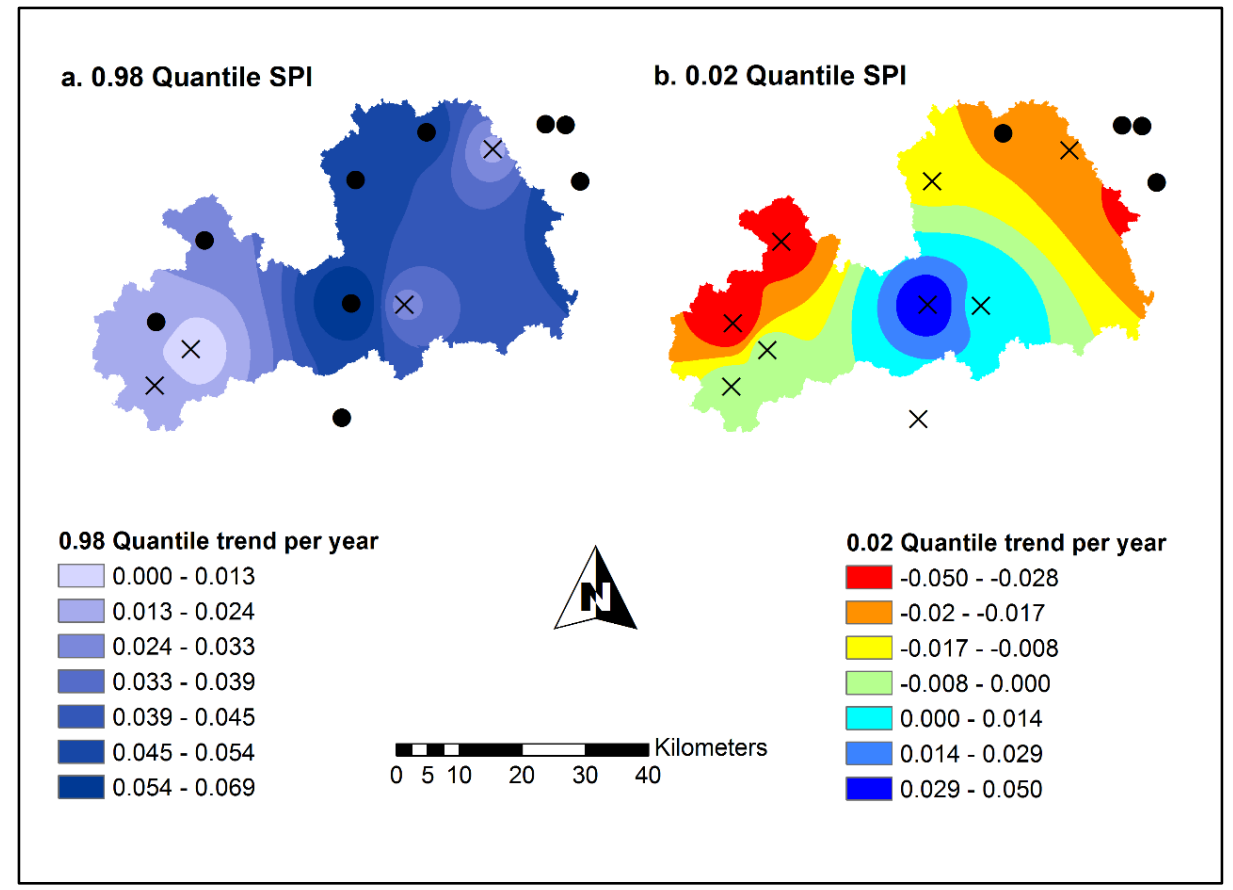

Fig. 5-12 Spatial distribution of the linear trend of annual SPI value for 0.98 and 0.02 quantile over Dee river catchment (circular dots represent significant trend points)

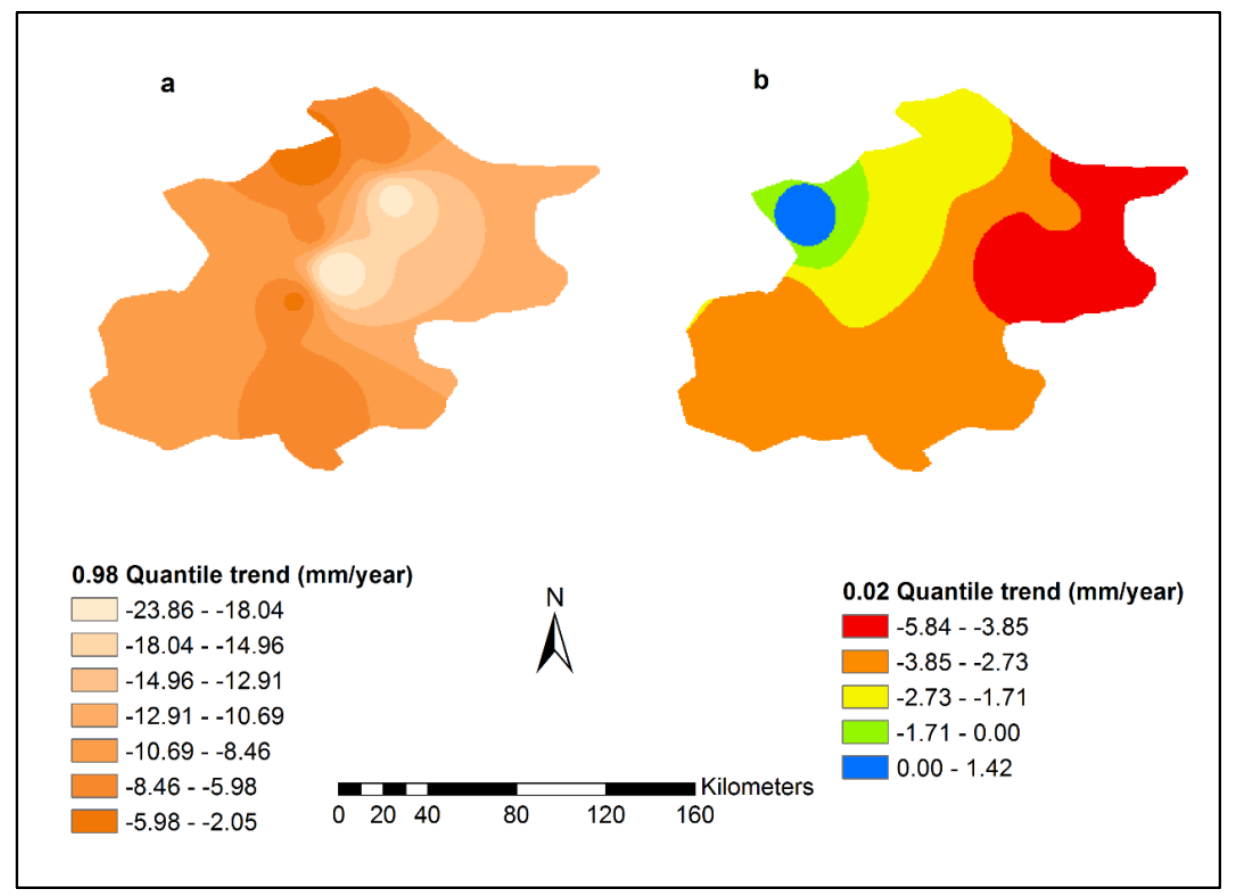

Fig. 5-13 Spatial distribution of the significant linear trend of annual precipitation over Beijing metropolitan area 
In a similar approach, the annual rainfall records for the metropolitan area of Beijing from the 45 rain gauges were utilised upon which the linear trend of 0.98 and 0.02 quantiles are produced at each rain gauges, before being interpolated over the area of study using Inverse Distance Weighted (IDW) method as revealed in Fig. 5-13. Unlike the Dee catchment, the pattern of the Beijing area shows remarkably decreasing trends for both lower and upper quantile (except the small part in the northeast). It indicates even for wet years; the precipitation is decreasing. What is even more remarkable is that such decreasing trends are more evident in the urban area (south and southeast). Urbanisation might be another important factor when it comes to the impact on annual precipitations as reported by Song et al. (2014).

Table 5-3: Slope and p-value of trend results of the areal average rainfall over the metropolitan area of Beijing

\begin{tabular}{cccc}
\hline Time & Quantile & Gradient & p-value \\
\hline Yearly & 0.98 & -2.065 & 0.02041 \\
& 0.02 & 0.023 & 0.97588 \\
July & 0.50 & -1.402 & 0.40441 \\
& 0.98 & 1.935 & 0.28137 \\
& 0.02 & 0.986 & 0.49443 \\
January & 0.50 & -1.658 & 0.01651 \\
& 0.98 & -0.137 & 0.33590 \\
& 0.02 & 0.000 & 1.00000 \\
Summer & 0.50 & -0.019 & 0.28897 \\
(JJA) & 0.98 & 1.280 & 0.54863 \\
& 0.02 & -3.034 & 0.00699 \\
Winter & 0.50 & -2.818 & 0.09819 \\
(DJF) & 0.98 & 0.047 & 0.81942 \\
& 0.02 & 0.003 & 0.91190 \\
& 0.50 & -0.091 & 0.35954
\end{tabular}

For Beijing, the monthly areal rainfall over the period of 1960-2012 is analysed with regard to its annual rainfall (after aggregation), typical winter and summer months (January and July) and two seasons (DJF and JJA). The linear quantile regression fits 
with different quantiles are included in Table 5-3 followed by Fig. 5-14 showing the slopes of these fits as well as the test results of significance. Above all, an apparent decrease trend of 0.98 quantile of both annual and summer rainfall is identified.

Annual Rainfall

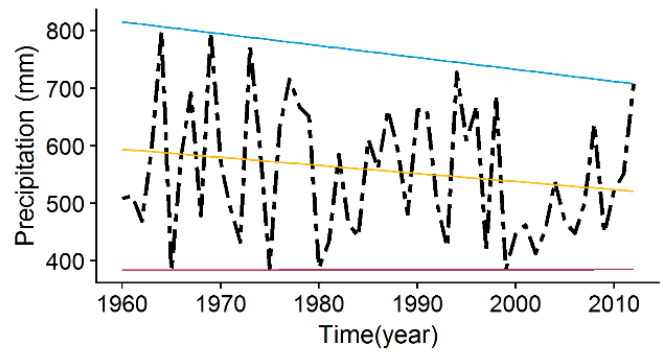

January Rainfall

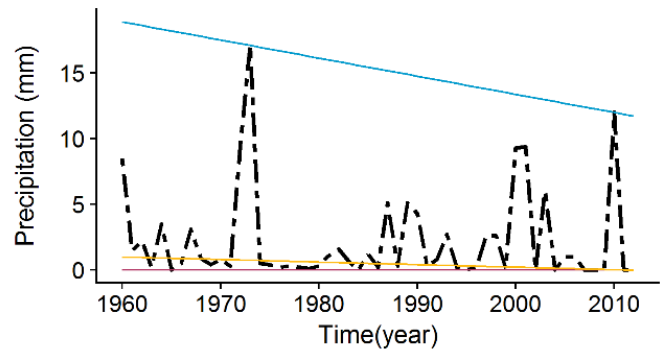

Winter Rainfall

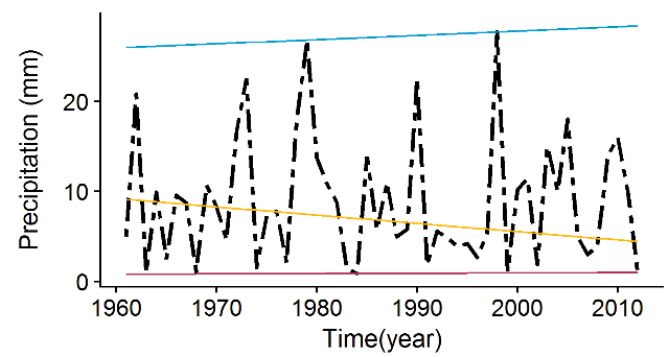

July Rainfall

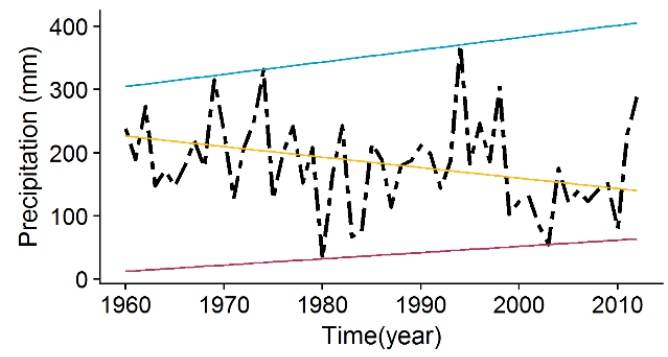

Summer Rainfall

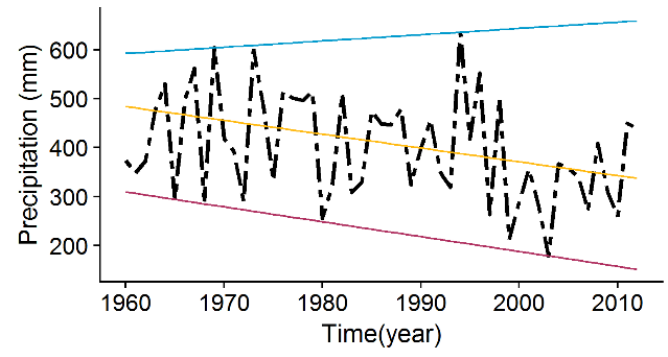

Fig. 5-14 Linear quantile regression trends for the areal average precipitation for flooding (tau=0.98), drought ( $\mathrm{tau}=0.02$ ) and mean (tau=0.50) conditions over Beijing area

For the month of July while in general decreasing trends are found for a range of different quantiles (lower than 0.98), the trend of 0.98 quantile does show an ascending pattern, although this trend is not statistically significant, due to the fact that there are only a few years in the late 1990s in which July witnessed a higher 
amount of monthly rainfall. This revelation is essential as it implicates a more volatile climate. For the lower end of the quantiles, none of the trends of 0.02 quantiles appears to be significant, which is also coincident with rather flat gradients.

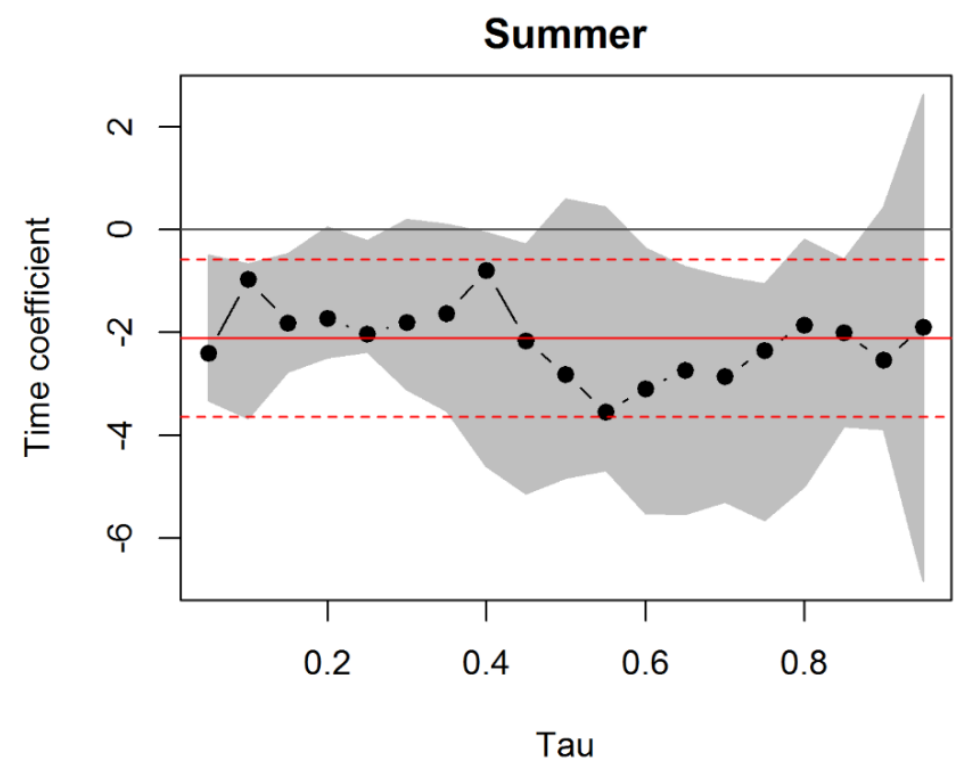

(a)

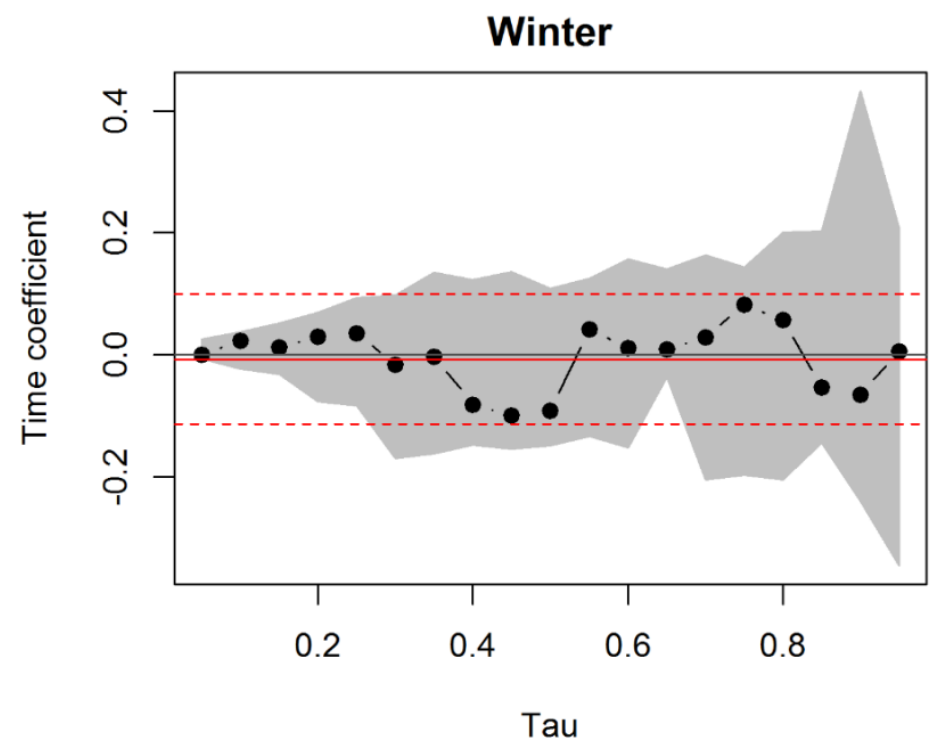

(b)

Fig. 5-15 Confidence band of the gradient ( $\mathrm{mm} /$ year) of the fitted lines using summer (a) and winter (b) seasons in Beijing (The horizontal axes are quantiles and the vertical axes refer to the gradient of the trend lines; the red lines represent the confidence bands of the fits using ordinary linear regression) 
The variation of trends conditional on the selected quantiles is shown in Fig. 5-15. The uncertain bands of the slopes reveal that, for summer, there is an increasing trend for quantiles below 0.5 and decreasing for those above 0.5 , but the trends seem to go up for quantiles larger than 0.8. The implication is that overall the summer rainfall tends to be more stable around its median, the heavy rainfall events may become more extreme. For winter, the gradient tended to be flatter and centred around 0 . In view of the significance test, it is not yet decisive to conclude any significant trends for winters.

\subsubsection{River flow trend}

There are plenty of studies of trend analysis over observed time series such as river flows, for example, Luce and Holden (2009). The same procedure can indeed be applied to this catchment; however, it is the use of the semi-distributed hydrological model that makes it possible to study the distribution of water resources over space. In this case, instead of investigating the trends of the observed flow, quantile regression is used to analyse the trends of model-simulated quantities including:

1) River discharges at the outlets of sub-basins; and

2) Water yields of the sub-catchments. Water yield is defined as the water quantity that departs the sub-basin and contributes the river flow during the time step (Arnold et al., 2013). It can be calculated as follow:

$$
\begin{gathered}
W Y L D=S U R Q+L A T Q+G W Q-T L O S S \\
- \text { Pond abstraction }
\end{gathered}
$$

where:

WYLD: Water yield ( $\mathrm{mm} \mathrm{H}_{2} \mathrm{O} /$ day);

$S U R Q$ : Surface runoff generated in the watershed $\left(\mathrm{mm} \mathrm{H}_{2} \mathrm{O} /\right.$ day);

LATQ: Lateral flow contribution to streamflow in the watershed $\left(\mathrm{mm} \mathrm{H}_{2} \mathrm{O} /\right.$ day);

$G W Q$ : Groundwater contribution to stream in the watershed ( $\mathrm{mm}_{2} \mathrm{O} /$ day); and 
TLOSS: Transmission losses ( $\mathrm{mm} \mathrm{H}_{2} \mathrm{O} /$ day). The amount of water that losses from the main channel to enter the deep aquifer.

One has to ensure the trends produced for the simulated data close enough to those of the corresponding observed data, just as it is done for model performance checking (such as NSE, $\mathrm{R}^{2}$, etc.) before the simulated data are used. The comparisons between the two types of trends (of observed and simulated river flows) are conducted at Ponty-Capel gauge station (see Fig. 3-8, page 50) which has long enough records as in Fig. $5-16$.

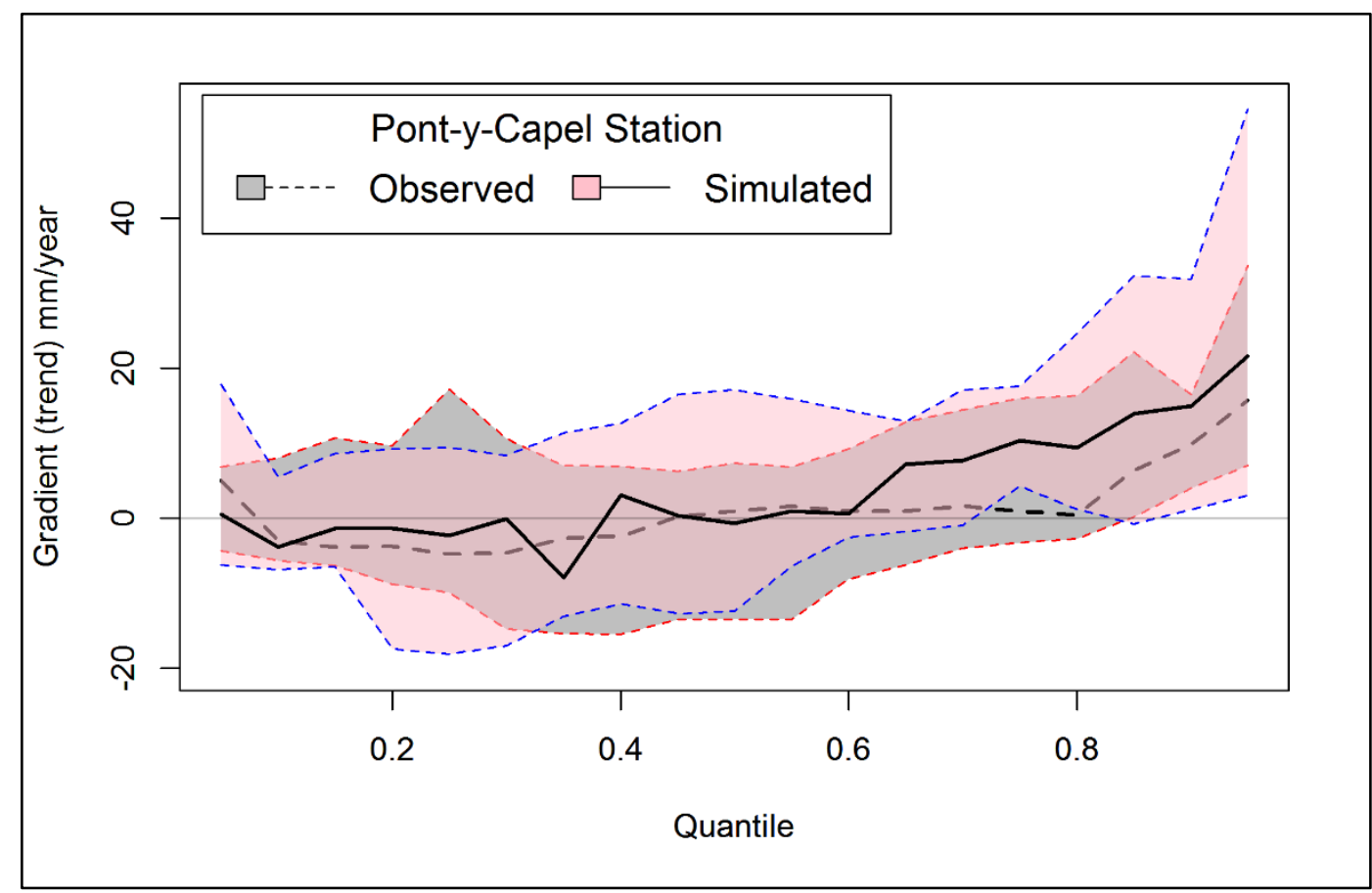

Fig. 5-16 Prediction bands of the gradient of observed and simulated river flow trends at Pont-y-Capel station

As seen in Fig .5-17, both trends are shown to be close with their mostly overlapping uncertainty bands. Three quantiles, $0.02,0.5$ and 0.98 are used to fit the trends of water yield from each sub-basin. The same quantiles are also adapted to analyse the river flow at the outlets of sub-basin for both scenarios. This process is followed by a comparison of the trend on the central reach of the river flow to quantify the effect of reservoir regulations on the river flow of Dee River. 


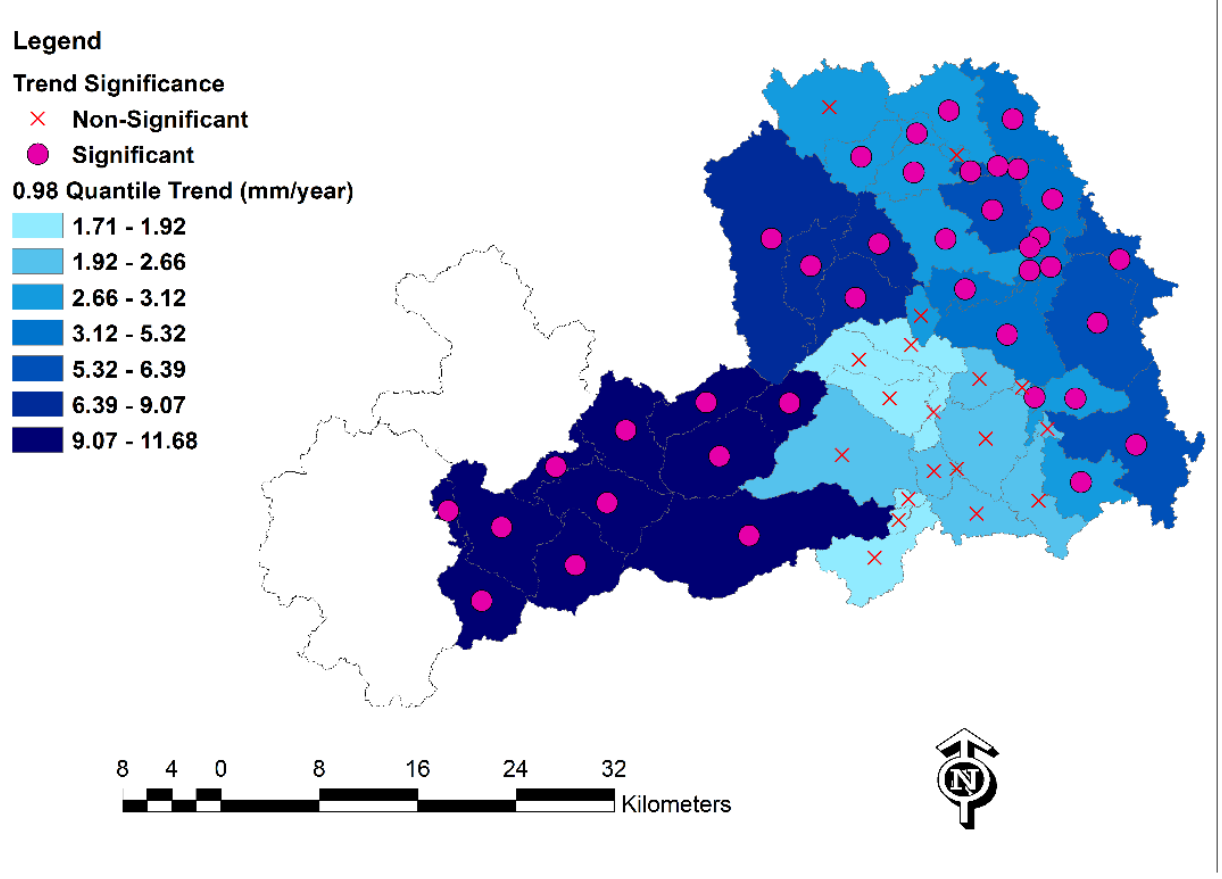

Fig. 5-17 Spatial distribution of the Water Yields trends at 0.98 quantile

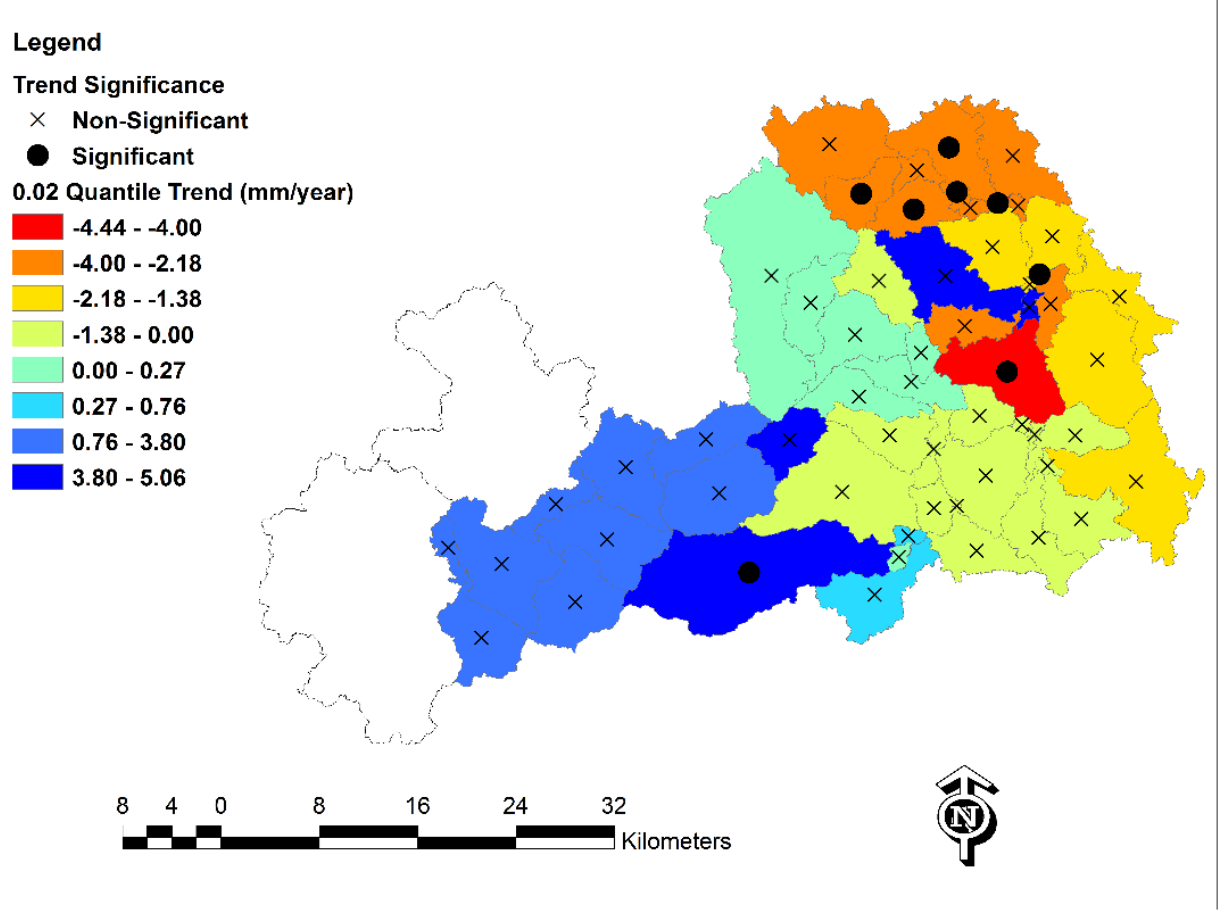

Fig. 5-18 Spatial distribution of the Water Yields trends at 0.02 quantile 
As to water yields, all sub-basins are analysed. The simulated time series of daily flows and water yields are firstly aggregated into yearly value before being fitted with a linear quantile regression model with three quantiles $0.02,0.5$ and 0.98 respectively. The 0.98 quantile shows a basin-wide positive trend with a range of 1.71-11.68 mm/year as illustrated in Fig. 5-17. Most trends are statistically significant at a confidence level of $95 \%$.

In comparison, the 0.02 quantile shows a mixed picture where negative trends are mainly observed in the downstream part of the river basin with a range of (-4.44)- ($0.29) \mathrm{mm} /$ year; whereas the upper stream part comes with a range of 0.76-5.06 $\mathrm{mm} /$ year positive trends as shown in Fig. 5-18. Unlike the trends of 0.98 quantile, in most sub-basins, the trends of 0.02 quantile are not statistically significant. The 0.5 quantile trend reveals a basin-wide increase trend except for a small region in the downstream area of the basin as shown in Fig. 5-19 with a significant trend at only one sub-basin in the south of the catchment.

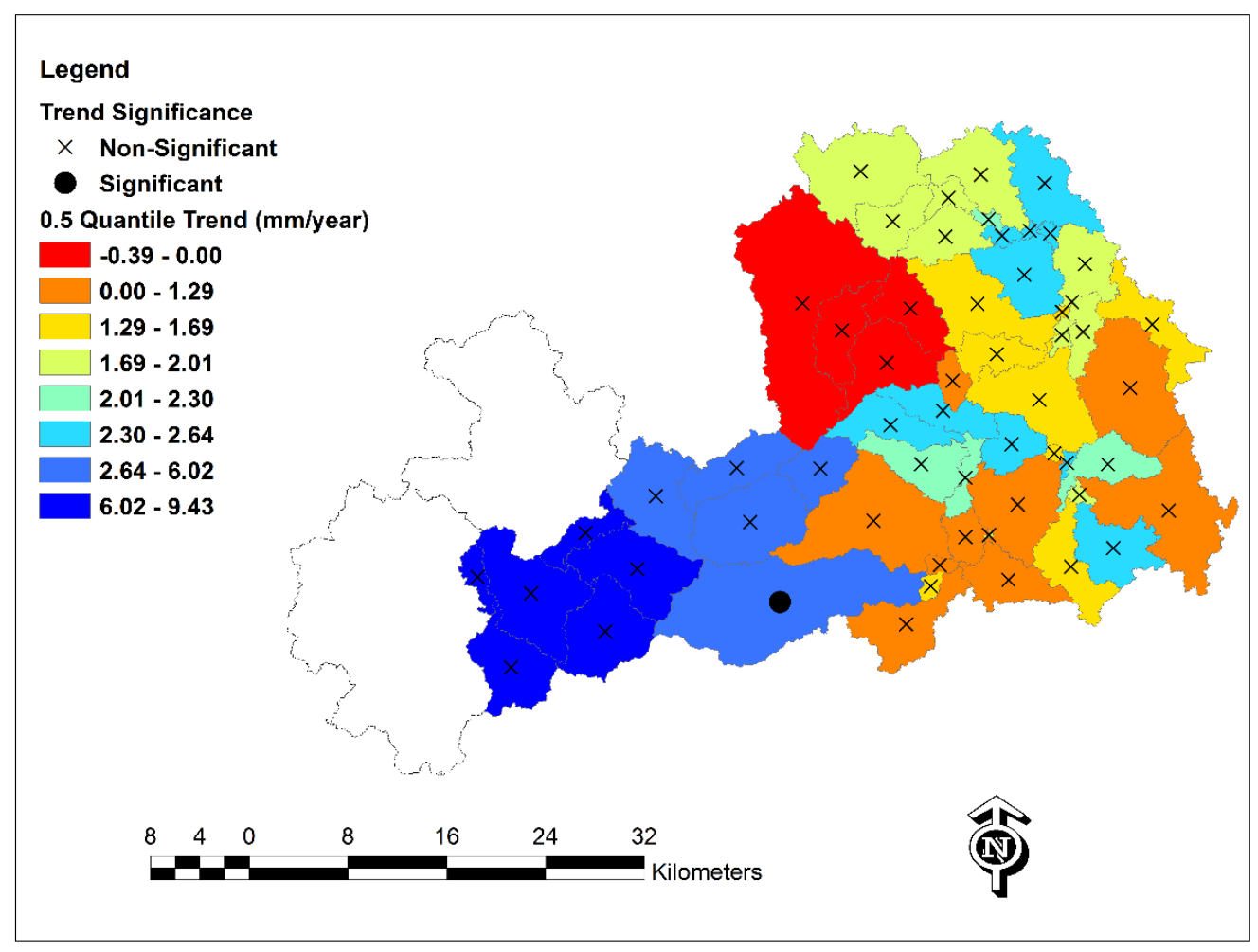

Fig. 5-19 Spatial distribution of the Water Yields trends at 0.5 quantile 
For the simulated discharge (river flow) at the outlets along the minor branches and mainstream, trends of 0.02, 0.98 and 0.5 quantiles are studied under both the managed and the natural scenarios. For both scenarios, the trends of 0.98 quantiles are all positive with a range of $0.03-15.45 \mathrm{Mm}^{3} /$ year for the highly-managed scenario as illustrated in Fig. 5-20 and 0.03-17.4 $\mathrm{Mm}^{3} /$ year for the natural scenario.

Again, at 0.02 quantile the results are more variable with negative values being mostly found in the downstream and positive trend values mainly appearing in the upstream region of the river basin. The ranges of trend values are -2.11 to 0.74 $\mathrm{Mm}^{3} /$ year for the 'real-life' scenario (Fig. 5-21) and -2.84 to $1.89 \mathrm{Mm}^{3} /$ year for natural flow scenario. Similarly, the 0.5 quantile shows a generally positive trend except for a small region in the downstream part that reveals in both highly managed scenario as in Fig. 5-22 and natural flow scenario. For both of these two scenarios, there is no significant trend.

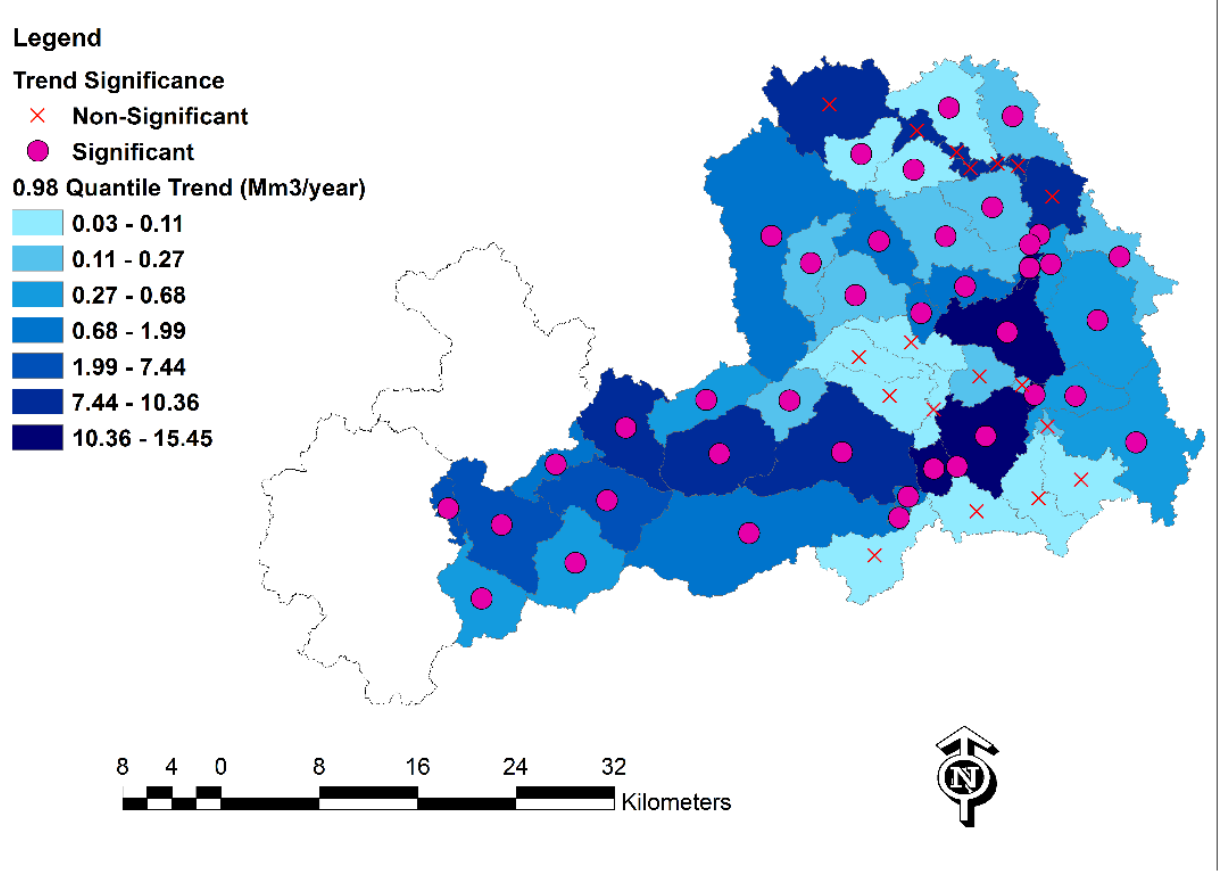

Fig. 5-20 Spatial distribution of the river flow trends at 0.98 quantile for highly managed watershed scenario 


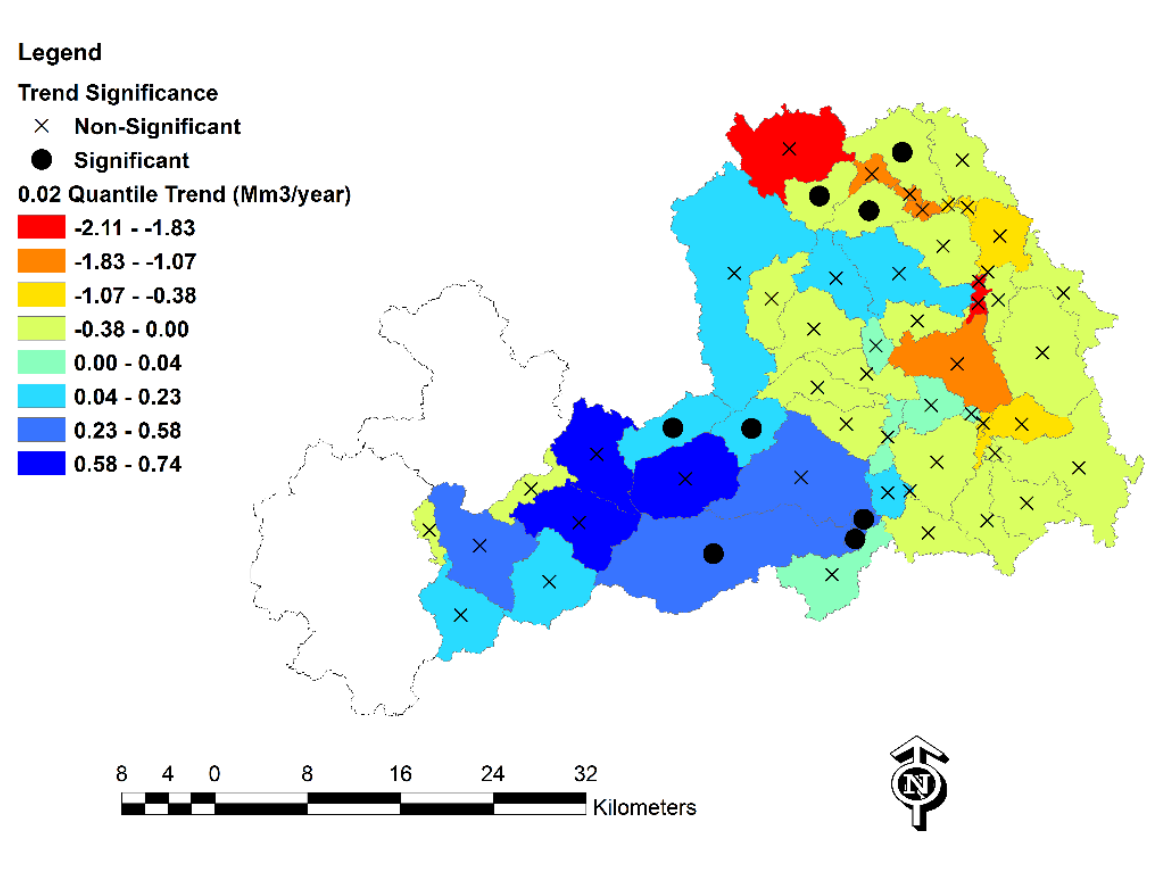

Fig. 5-21 Spatial distribution of the river flow trends at 0.02 quantile for highly managed watershed scenario

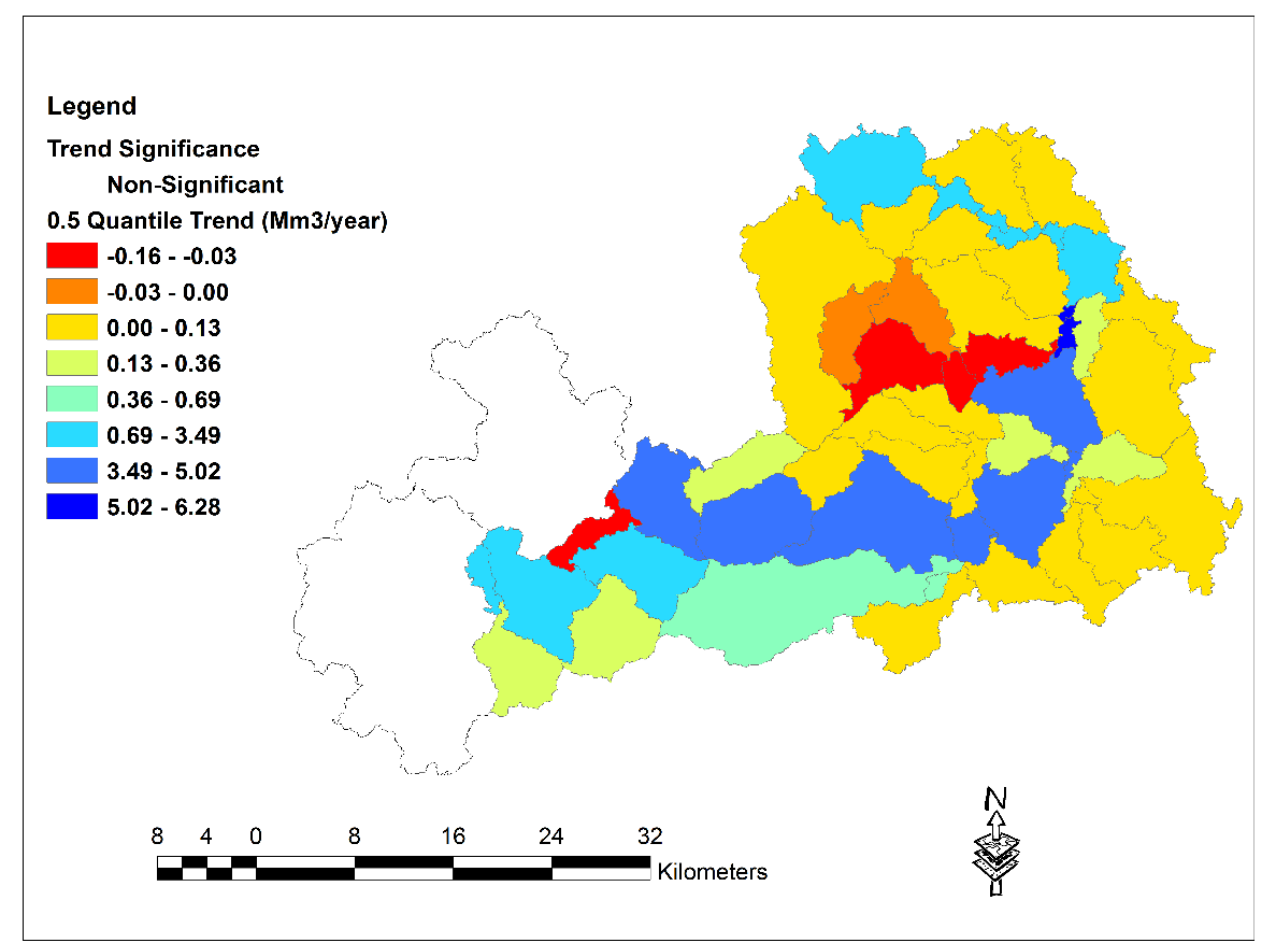

Fig. 5-22 Spatial distribution of the river flow trends at 0.5 quantile for highly managed watershed scenario 
The impact of flow control (river basin management) can be readily appreciated from Figures. 5-23, 5-24 and 5-25 for quantiles of 0.98, 0.02 and 0.5 respectively. The management practices affect significantly on the trends of both the high (0.98 quantile) and the low flow (0.02) as well as the medium flow condition (0.5 quantile). For 0.98 and 0.5 quantiles, the positive trends are less in the managed scenario. For 0.02 quantile (low flow condition), the management practices, clearly mitigate the strong negative trends in the downstream areas as compared with the natural scenario. Since the same water withdraw amounts are applied in both scenarios, the mitigation is shown to have effectively reduced the pressure on water supply. Such an impact is also reflected in the upper stream where the management practices have reduced the positive trend as well (Fig 5-24).

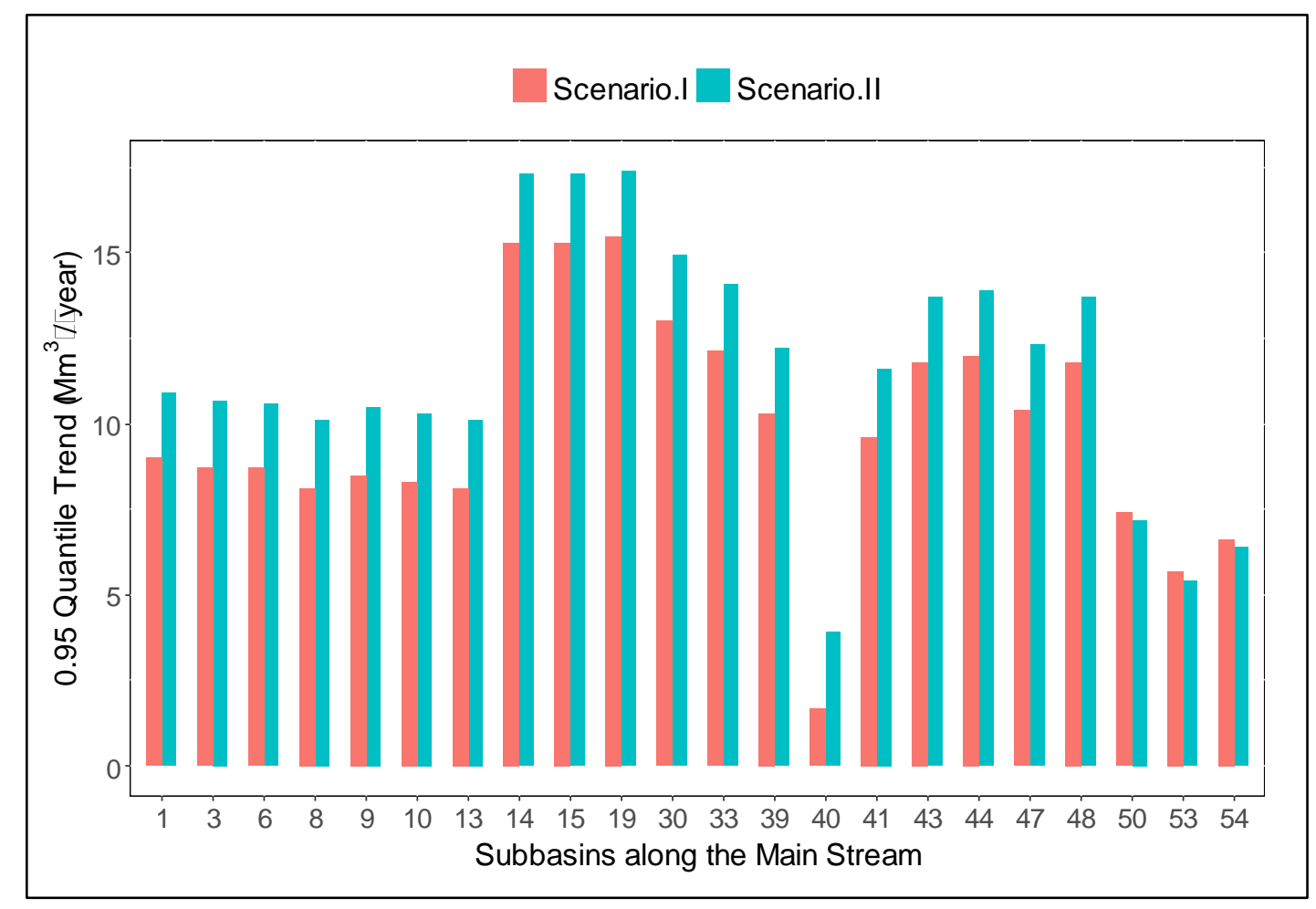

Fig. 5-23 Comparison of river flow trends of the sub-basins along the mainstream under Scenario I (highly-regulated) and Scenario II (natural-state) at 0.98 quantile 


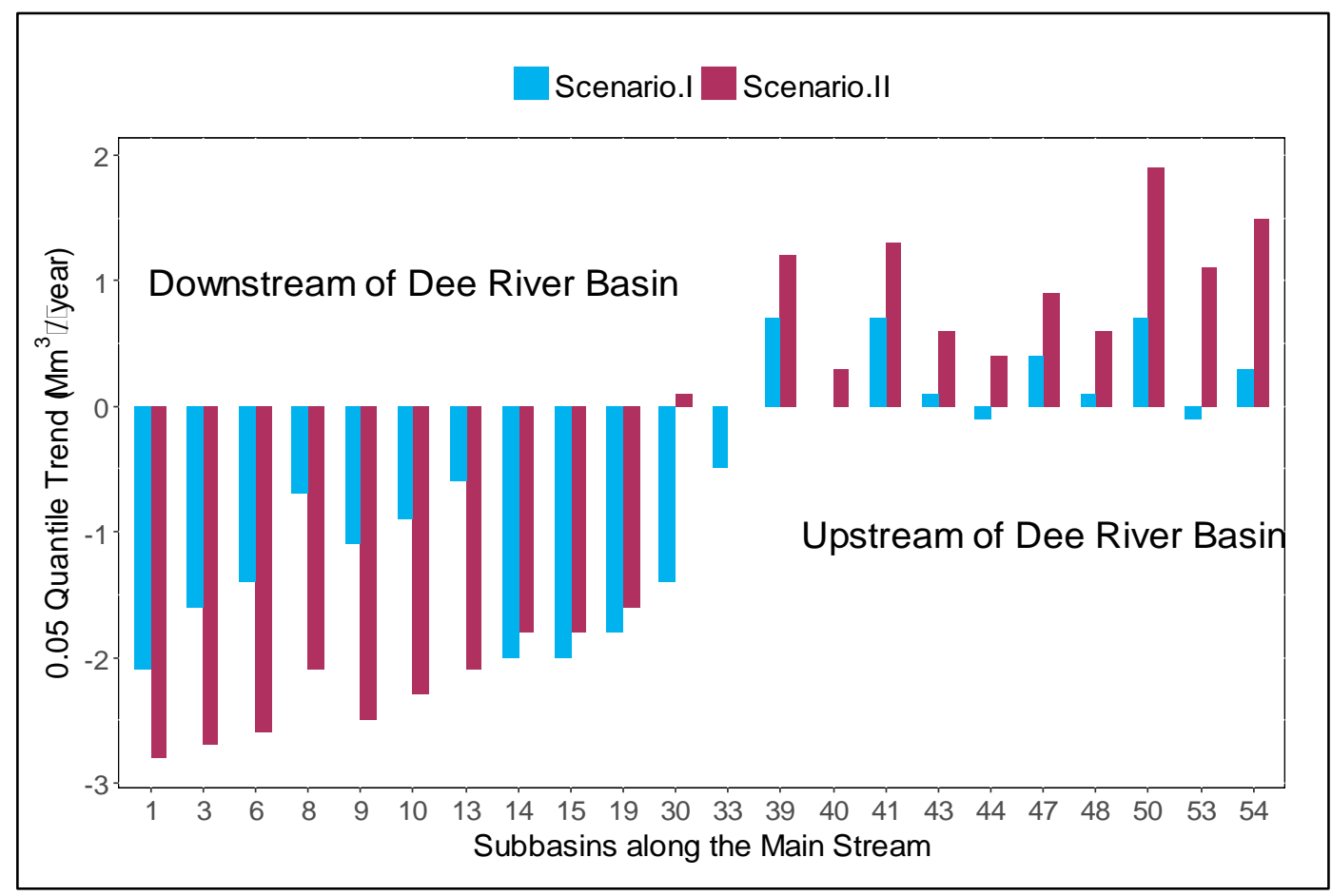

Fig. 5-24 Comparison of river flow trends of the sub-basins along the mainstream under Scenario I (highly-regulated) and Scenario II (natural-state) at 0.02 quantile

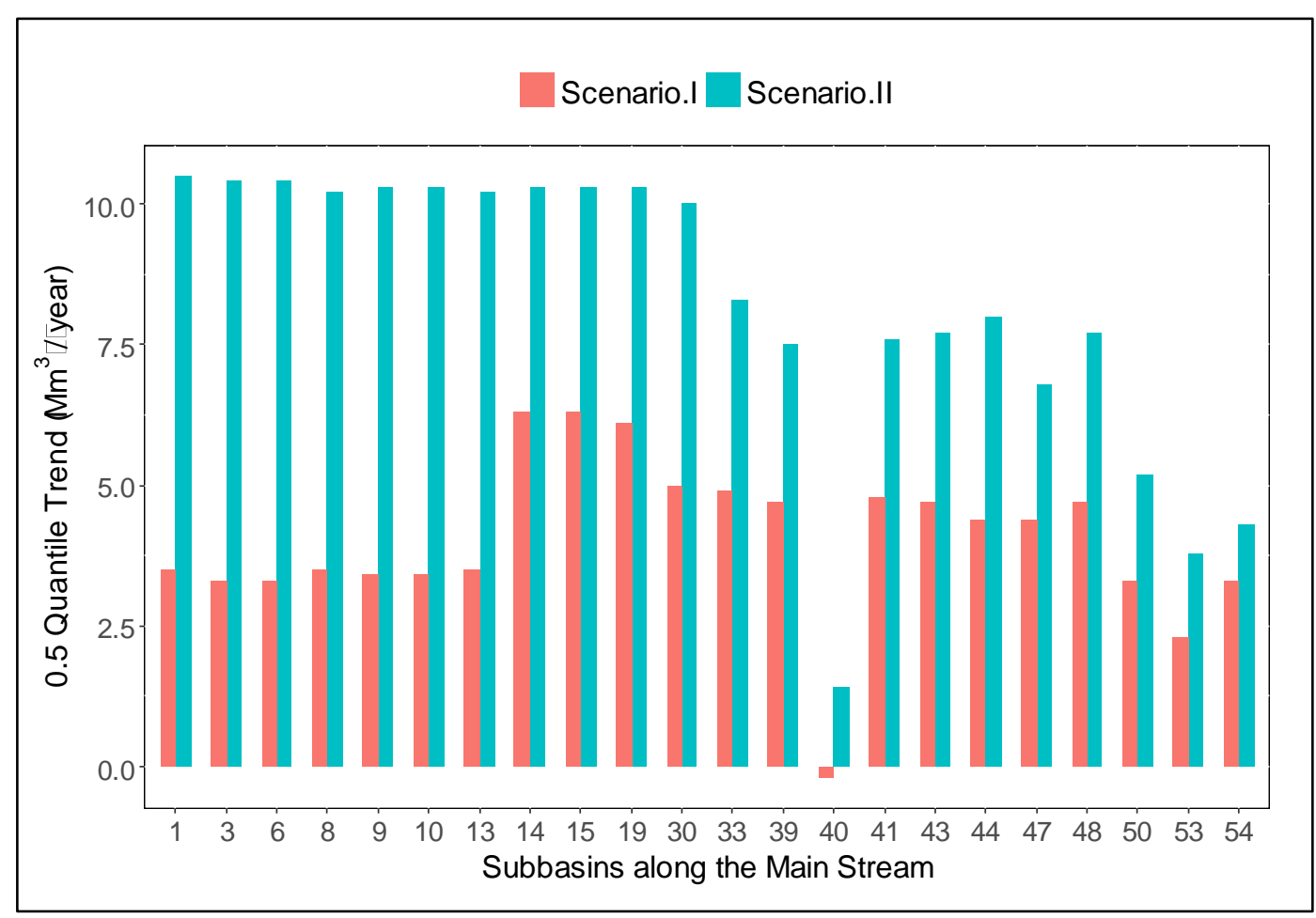

Fig. 5-25 Comparison of river flow trends of the sub-basins along the mainstream under Scenario I (highly-regulated) and Scenario II (natural state) at 0.5 quantile 


\subsection{Summary}

In this Chapter, a quantile regression-based method is demonstrated for analysing the trend in climatic variables. Long-term rainfall data from two drastically different climate regions is investigated focusing on the patterns of the data close to 'extreme' regimes, to link them to the events of interests. Two quantiles 0.98 and 0.02 are used to represent the extreme wet (hence flooding) and dry (droughts) conditions. The results are also spatially interpolated to study the trend variation in space.

In comparison with the commonly used linear regression method, it can be concluded that:

1) The QR based trend analysis can provide far more detailed information concerning the quantity in question. This is particularly useful for water managers who are more concerned with extreme values rather than the average one;

2) This method can help build a comprehensive picture of climatic variables regarding their variation over time at different magnitude/frequency;

3) The involvement of quantile brings an extra benefit to bridge the trend analysis with frequency, which implies great potential of its use in studying climate change impact on engineering design without being constrained by assumptions of data stationarity; and

4) It helps better to understand the climate change impact. As already shown, a decreasing trend in summer rainfall may still be accompanied with increasing severe storms in the same season.

Undoubtedly, more studies are needed to improve the method with respect to the problems found in this study, which include but are not limited to:

1) A better link between quantiles and event frequency (return period) in the context of trend analysis; 
2) The quantiles used in this study are not really 'extreme'. Reliable quantile regression for the higher quantile (e.g., 99\%) needs to be explored; and

3) The method needs to be refined further to be more reliable on lower quantiles. It has been found that most trends of quantile 0.02 are insignificant, but this may not be true as the lower end of the data suffer from larger errors and zero values may as well interfere with the process.

Furthermore, a quantile regression-based method is presented to study long-term trends of river flow and catchment water yields under the influence of water resources management practice. Three daily SWAT models are set up to simulate the hydrological processes in the Dee River catchment in the UK with flow control and water withdrawal process explicitly represented. Two scenarios (with and without flow control) are constructed to explore the impact of management practice. Further, two quantiles 0.02 and 0.98 are used to indicate high flow (wet) and low flow (dry) conditions that water managers are most concerned with. The 32-year simulated river flow and sub-basin water yields are analysed. The quantile of 0.5 is also considered as the medium flow and/or water yield.

The results show that such combination of quantile regression and semi-distributed hydrological modelling approach excels in presenting distributed, spatially focused trend information for extremely dry and wet scenarios, which can thoroughly address the needs of practitioners and decision makers in dealing with long-term planning and climate change. The representation of the management practice such as flow control and water withdraw in the modelling process can help reveal the impact from the latter, and as such lays a foundation for further study on how various management practice can mitigate the effect from other sources such as climate change on catchment water resources management.

For the study area, the Dee River basin, it has been shown that the 0.98 quantile has an increasing trend for both 'real-life' and natural flow scenario with a significant trend at most of the river basin. Additionally, the result reveals that the management practices tend to reduce the floods in the catchment. For the 0.02 quantile, both 
positive (mainly upstream) and negative (downstream) trends exist for the two scenarios, albeit most of them are statistically insignificant. The comparison of the two scenarios indicates that the management practices are indeed able to mitigate strong decrease trends in the downstream.

The main finding is that trends of low quantile 0.02 are mostly insignificant necessitates further study. As the trend analysis is conducted over the simulated data, the performance of the model, especially its representativeness of high and low flow conditions may directly affect the results hence the conclusion. More vigorous quality control of the modelling process may need to be in place and further studies are also recommended. 


\section{Chapter 6: Coupled Surface-Groundwater Modelling}

Groundwater is one of the main components of the hydrological cycle but has not been well modelled by surface hydrological models such as SWAT. Accurate simulation of both land surface and groundwater hydrological processes in river catchments is a fundamental step for integrated water resources management, particularly for watersheds where both surface water and groundwater resources are used conjunctively. In this chapter, a coupled land surface model (SWAT) and groundwater flow model (MODFLOW) are used to model a complex river catchment - the Dee River catchment to improve the performances of both models otherwise used separately, hence serving the IWRM goals of optimising the conjunctive use of surface and groundwater. The model can also be used to evaluate the sensitivity of stream flows to changing climate, groundwater extraction, and land use alternations. The results show that the coupled model can improve river flow simulation especially baseflow simulation while significantly improving the overall water balance and the low flow simulations.

\subsection{Introduction}

In recent decades, there has been growing stress of surface and groundwater resources around the world (e.g. Aeschbach-Hertig and Gleeson, 2012; Taylor et al., 2013). Owing to climate change and population growth, water resources managers, are aware of the balance of increasing demand to provide reliable water supplies to among different stakeholder. Typically, as the surface water supplies are exhausted, users increasingly turn to groundwater to enhance the supplies and mitigate the likely drought impacts (Schoups et al., 2006). It is estimated that $40 \%$ of the world's food production is irrigated using both surface and sub-surface water resources (McCray, 2001).

Integrated Water Resources Management (IWRM) at catchment level relies heavily on the use of computer model simulations that capture the underlying hydrological processes and surface water/groundwater allocations. Some examples of models are: The Soil and Water Assessment Tool (SWAT; Arnold et al., 1998) and the Modular 
Three-Dimensional Finite-Difference Groundwater Flow (MODFLOW; Niswonger et al., 2011) which are well-tested and widely-used surface and groundwater models (e.g. Golmohammadi et al., 2017; Milzow and Kinzelbach, 2010), respectively. However, these models represent the physical world (i.e., model spatial discretisation and process simulation) differently and each is limited to its simulation domain, each having advantages and disadvantages when simulating biophysical processes and using computational resources (Guzman et al., 2015).

The SWAT model only simulates the shallow groundwater dynamics above a restricted layer (SWAT model lower boundary domain). Percolation below the impervious layer, which is set at a maximum value of $6 \mathrm{~m}$ below the ground surface is assumed lost out of the system (Luo et al., 2012). SWAT simulates both the surface and the shallow aquifer processes based on hydrological response units (HRUs), which are conceptual units of homogeneous land use, management, slope, and soil characteristics that extend below the surface to a soil profile depth (Arnold et al., 1998). Even though SWAT has its own module for groundwater components, it is lumped and as a result parameters such as hydraulic conductivity cannot be spatially represented (Arnold et al., 1993). Furthermore, the SWAT model creates difficulties when expressing the spatial distribution of groundwater levels and recharge rates.

On the other hand, MODFLOW simulates flow processes occurring at the continuum volume in the saturated zone defined by three-dimensional cells (groundwater domain) and the hydrogeological properties. MODFLOW simultaneously solves the groundwater flow differential equation using the finite difference approach, and integrates groundwater systems with other hydrological sub-system components (e.g. vadose zone, surface drainage, transport phenomena,...etc.) through the incorporation of 'packages' using a gridded spatial discretisation. However, it does not directly account for hydrologic processes that occur on the surface or in the root zone.

One of the essential characteristics of an efficient groundwater model is the accuracy of recharge rates within the input data. The standard groundwater flow model conducted via MODFLOW often ignores the accuracy of the recharge rates, the 
primary input in MODFLOW, that are required to be estimated into the model. Consequently, there is considerable uncertainty in the simulated groundwater flow results (Guzman et al., 2015).

Therefore, a common practice is to assume lumped percolation fluxes as a percentage of precipitation and then optimise the value during the calibration process. Whereas the groundwater model calibrated for recharge can provide reasonably good groundwater level predictions, it is possible that the user may get the right answer for the wrong reasons (Kirchner, 2006) because this approach fails to account for spatial variability in recharge rates as a result of varying land use, irrigation and agronomic practices implemented on the surface domain. Moreover, this approach may misrepresent transport of nutrients moving to the groundwater domain for the same reasons.

For that reason, an integrated SWAT and MODFLOW is important to better spatially represent feedback fluxes within the surface and groundwater domains. It will improve simulation of the impacts of long-term stressors, such as:

1) Climate change impact studies (e.g. Wheeler and Von Braun, 2013);

2) Management of water irrigation (e.g. Playan and Mateos, 2006); and

3) Land use change scenarios (e.g. Chu et al., 2013).

Many researchers have reported in the literature that SWAT models were integrated with other models such as:

1) Improve simulations of riparian buffer zones (SWAT-REMM; Ryu et al., 2011);

2) The simulation of sediment and hydrodynamic flow (SWAT-SOBEK; Betrie et al., 2011);

3) The management of stormwater (SWAT-SWMM; Kim et al., 2011) within the framework of OpenMI integration (Gregersen et al., 2007); and

4) Surface and sub-surface water processes (SWAT-MODFLOW; Guzman et al., 2015; Bailey et al., 2016, 2017; Kim et al., 2008). 
The key objective of model integration is to bridge the gap of multi-disciplinary knowledge to support the quantitative capacity for the rigorous assessment of hypotheses and system response under dynamic scenarios (Arnold, 2013). However, when it comes to more complex IWRM scenarios where both surface and groundwater abstractions are substantially utilised, the coupling of traditionally surface water orientated hydrological models such as SWAT, with a dedicated groundwater model such as MODFLOW has become a focal research area. More recently, progress has been made (e.g. Bailey et al., 2017) to develop a series of tools that can conveniently couple SWAT with MODFLOW on a daily time step.

Many applications have also been reported with linked SWAT and MODFLOW codes such as (Guzman et al., 2015; Kim et al., 2008; Bailey et al., 2016). Typical model integration includes using SWAT-calculated soil deep percolation as a recharge for MODFLOW and using MODFLOW-simulated groundwater-surface water interaction (i.e. groundwater discharge to stream; stream seepage to aquifer) as input for SWAT (Bailey et al., 2016).

Among hydrological processes, the understanding the characteristics of baseflow could be a step forward for the better estimation of groundwater recharge that has highest priorities for sustainable water resources management. Eckhardt (2008) points out that Baseflow is a division of river flow that gradually responds to rainfall which is typically connected with groundwater storage discharge. Under low-flow conditions, the detailed information of baseflow is useful for the evaluation of streamflow forecasting, allocating water resources and design of hydropower plants (Tallaksen, 1995). When, where, and how much streamflow can be attributed to groundwater discharge is therefore practically significant (Luo et al., 2012). As a result, baseflow is an essential component of the hydrological simulation.

SWAT uses a conceptual one-reservoir (shallow aquifer storage) method to simulate baseflow (Luo et al., 2012). It divides the groundwater component into two aquifer systems (Arnold et al., 1993):

1) The shallow aquifer that contributes baseflow to streams within the watershed; and 
2) The deep aquifer that contributes baseflow to streams outside the basin and can be considered lost from the system.

Many researchers reported and agreed that SWAT model has a weaker baseflow simulation such as Srivastava et al. (2006); Chu and Shirmohammadi (2004); Wu and Johnston, (2007) and Luo et al. (2012).

In this Chapter, the focus is set on the overall water balance and low flow simulation (Baseflow) in a complex river catchment, Dee watershed, based on a well-performing SWAT model that simulates high and peak flow satisfactorily throughout the basin. This chapter demonstrates the methods of linking SWAT and MODFLOW for the Dee River catchment and preliminary results and analysis. Finally, a conclusion is drawn with recommendations for further studies.

\subsection{Materials and methods}

\subsubsection{Groundwater data}

The data used to create the SWAT model are presented and described in (section 3.3, page 37 in chapter 3). The data used to create the groundwater flow model, MODFLOW, are demonstrated in this section.

There are also three categories of data collected to model catchment, namely:

1) The static dataset, such as DEM (used as the elevation of ground surface), depth of groundwater from the ground surface (used to estimate initial groundwater head), aquifer designation data and soil type map (for horizontal permeability values) that are assumed to be static over the study period;

2) The historical observations of daily groundwater level; and

3) The operational data of licensed groundwater abstraction data that represent management practice.

Most data used are subjected to an academic license. The summary of data is exemplified in Table 6-1. 
Table 6-1: Collected data for the MODFLOW model

\begin{tabular}{lll}
\hline Data & Resolution & Source \\
\hline Groundwater depth & $50 \mathrm{~m}$ & British Geological Survey, BSG \\
Soil Map & $50 \mathrm{~m}$ & British Geological Survey, BSG \\
Aquifer designation & $50 \mathrm{~m}$ & British Geological Survey, BSG \\
Groundwater level & Daily (1975-2014), & British Geological Survey, BSG \\
& One monitoring well & \\
Groundwater withdraws & 37 licensed wells & Natural Resources Wales, NRW
\end{tabular}

\subsubsection{SWAT model}

The calibrated daily SWAT model in chapter 3 was used to be integrated with the MODFLOW. The simulation period of 1992-2003 with a 3-years warm-up period to make hydrological cycle fully operational (1995-2000 calibration period) and the rest for the validation. Six streamflow gauge station will be employed to test the performance of the simulated river flow of both SWAT model and coupled SWATMODFLOW as revealed in Fig. 3-8.

In SWAT model, water routed through channel system to the gauges consists of four components: direct surface runoff, lateral flow from unsaturated soil profiles, drainage from tiles and baseflow from underground storage (Ly et al., 2011). The modelling of the direct surface runoff, the lateral soil flow and the tile drainage are described in detail in theoretical documents of SWAT model (Neitsch et al., 2011). The baseflow simulation will be focused on henceforth.

SWAT model distinguishes the underground storage into two parts, the shallow aquifer and the deep aquifer. The shallow aquifer receives recharge from the unsaturated soil profile percolation. An exponential decay weighting function is utilised to account for the time delay in aquifer recharge once the water exits the soil profile (Neitsch et al., 2011). The delay function accommodates situations where the recharge from the soil zone to the aquifer is not instantaneous, i.e. one day or less. The recharge to aquifer on a given day is calculated as below: 


$$
\begin{aligned}
W_{\text {rchrg,i }}=[1 & \left.-\exp \left(-\frac{1}{\delta_{g w, s h}}\right)\right] W_{\text {seep }} \\
& +\exp \left(-\frac{1}{\delta_{g w, s h}}\right) W_{\text {rchrg,i-1 }}
\end{aligned}
$$

where:

$W_{\text {rchrg }}$ is the amount of recharge entering the aquifers $\left(\mathrm{mm} \mathrm{H}_{2} \mathrm{O} /\right.$ day);

$\delta_{g w, s h}$ is the delay time of the overlying geologic formations (days); and

$W_{\text {seep }}$ is the total amount of water exiting the bottom of the soil profile $\left(\mathrm{mm} \mathrm{H}_{2} \mathrm{O}\right.$ / day);

The subscriptions "seep" indicates seepage water exiting bottom of the unsaturated soil profile; "rchrg" means recharge, $i$ is the sequential number of days, and "sh" indicates the shallow aquifer storage. A fraction of the total daily recharge can be routed to the deep aquifer. The amount of water diverted from the shallow aquifer due to percolation to the deep aquifer on a given day is given by:

$$
W_{\text {seep }, d p, i}=\beta_{d p} W_{\text {rchrg, } i}
$$

where:

$\beta_{d p}$ is a coefficient of shallow aquifer percolation to deep aquifer, and the subscription " $d p$ ": indicates deep aquifer. The amount of recharge entering the shallow aquifer is:

$$
W_{\text {rchrg }, \text { sh }, i}=W_{\text {rchrg,i }}-W_{\text {seep }, d p, i}
$$

Baseflow generated from the shallow aquifer on a given day $i$ under the influence of recharge is specified as below (Neitsch et al., 2011): 


$$
\begin{aligned}
Q_{b, s h, i}=Q_{b, s h, i-1} \cdot & \exp \left(-\alpha_{g w, s h} \cdot \Delta t\right) \\
& +W_{r c h r g, s h, i} \cdot\left[1-\exp \left(\alpha_{g w, s h} \cdot \Delta t\right)\right]
\end{aligned}
$$

where $Q_{b, s h, i}$ is the baseflow from the shallow aquifer on day $i\left(\mathrm{~mm} \mathrm{H}_{2} \mathrm{O} /\right.$ day), and "b" indicates baseflow, and $\Delta t$ is the step time length. Daily time-step is used in this study. When only one reservoir is used, the baseflow is equal to that from the shallow aquifer:

$$
Q_{b, i}=Q_{b, s h, i}
$$

SWAT assumes that water entering the deep aquifer is not considered in the future water budget calculations and can be considered lost from the system (Neitsch et al., 2011).

\subsubsection{MODFLOW model}

MODFLOW (McDonald and Harbaugh, 1988; Niswonger et al., 2011) is a threedimensional, physical-based, distributed finite differences groundwater flow model for the variably saturated sub-surface system. A recent addition to MODFLOW is the Newton-based solver algorithm that better satisfies the complex non-linear drying and re-wetting of grid cells in unconfined groundwater system (Niswonger et al., 2011), a problem with previous versions. Available processes to be simulated in MODFLOW include groundwater recharge, vadose zone percolation, evapotranspiration, pumping, discharge to sub-surface drains, river-aquifer interactions (Bailey et al., 2016).

However, most applications are limited to investigating management and climate effects on groundwater and surface-groundwater interaction as MODFLOW does not simulate surface processes such as land-atmospheric interactions, infiltration and surface runoff, nutrient cycling and transport, plant growth and the impact of management practice on agricultural systems (Bailey et al., 2016). Darcy's law governs the flow rate. It can simulate steady and non-steady flows in a saturated system, in which aquifer layers can be confined, unconfined, or a combination of 
confined and unconfined. The following partial differential equation describes threedimensional groundwater flow:

$$
\frac{\partial}{\partial x}\left[k_{x x} \frac{\partial h}{\partial x}\right]+\frac{\partial}{\partial y}\left[k_{y y} \frac{\partial h}{\partial y}\right]+\frac{\partial}{\partial z}\left[k_{z z} \frac{\partial h}{\partial z}\right]-w=S_{S} \frac{\partial h}{\partial t}
$$

where:

$k_{x x}, k_{y y}$ and $k_{z z}$ : are the hydraulic conductivities along the $\mathrm{x}, \mathrm{y}$, and $\mathrm{z}$ coordinate axes parallel to the major axes of hydraulic conductivities;

$h$ : is the potentiometric head;

$w$ :is a volumetric flux per unit volume representing sources ( $W$ is negative) and/or sinks ( $W$ is positive) of water;

$S_{s}:$ is the specific storage of the porous medium; and

$t$ : is time.

$k_{x x}, k_{y y}, k_{z z}$ and $S_{s}$ are functions of space $(x, y, z)$ and $W$ is a function of space and time $(x, y, z, t)$ (Todd and Mays, 2005). In MODFLOW, an aquifer system is replaced by a discretised domain consisting of an array of nodes and associated finite difference blocks (cells) (Chiang and Kinzelbach, 1998). In this chapter, groundwater flow is simulated using the MODFLOW-NWT model including the following package:

1) Basic package (. bas);

2) Discretisation package (. dis);

3) River package (. riv);

4) Well package (. wel);

5) Upstream weighted package (. upw);

6) Recharge package (. rch); and

7) Newton Solver package (. nwt).

In this study, the Geographical user interface GUI such as Visual MODFLOW Flex, Groundwater Vistas and GMS are not used because most of them are commercial and very expensive. Instead, a model is created using ArcMap and Excel with some Visual Basic coding. For the simplifying the interaction between SWAT's HRUs and 
MODFLOW grid cells, the aquifer system in this study was set up as a one-layer (taking the average value of two layers for the permeability values), unconfined aquifer and unsteady groundwater flow model (the necessary condition to link the model to SWAT hereafter). Moreover, the model divides the cells into $200 \mathrm{~m} \times 200 \mathrm{~m}$ (coefficient of DEM cell size) and accordingly, the aquifer was discretised into a grid of 241 rows and 317 columns. The topographical surface assigned as the top layer of the model was interpolated from the Digital Elevation Model (DEM).

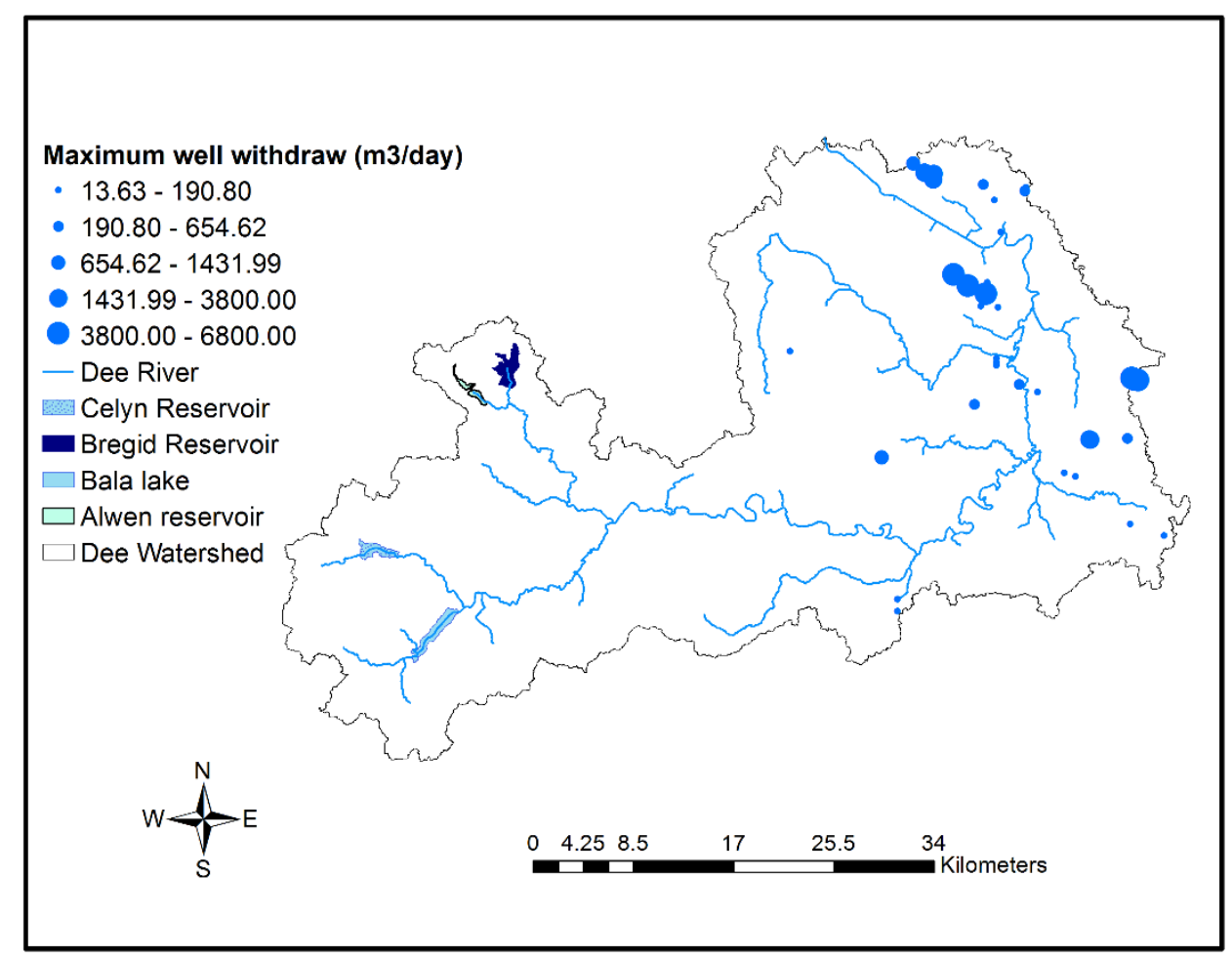

Fig. 6-1 Location of the license withdraw wells used in MODFLOW model

A total number of 37 licensed wells are represented in this study with maximum water withdraw of 14-6800 $\mathrm{m}^{3} /$ day as shown in Fig. 6-1. One unconfined layer of soil is used in the model with a single stress period (considering maximum well withdraw during the whole period of the simulation) to make SWAT-MODFLOW run faster.

\subsubsection{SWAT-MODFLOW coupling}

SWAT model is principally limited in terms of dealing with groundwater flow because of its lumped nature. On the other hand, MODFLOW has difficulty in 
calculating the distributed groundwater recharge that is the primary input for the groundwater model. Consequently, it is promising for the hydrological variables to be realistically computed if an HRU-based groundwater recharge is used for input data in MODFLOW and the groundwater flow between the aquifer and the stream is calculated and exchanged to SWAT, then the spatiotemporal features in the catchment will be adequately represented (Kim et al., 2008).

The coupled SWAT-MODFLOW framework (Bailey et al., 2017) combines an updated version of the SWAT model (SWAT 2012, revision 627) with MODFLOWNWT. In this framework, SWAT simulates land surfaces processes, crop growth, instream processes and soil zone processes. Meanwhile, MODFLOW-NWT simulates three-dimensional groundwater flow and all associated sources and sinks (e.g. recharge, pumping, discharge to tile drains and interaction with stream networks). Both modelling codes are combined into single FORTRAN code that is compiled and run as a single executable file.

The basic process of linking SWAT and MODFLOW models is to pass HRUcalculated deep percolation (i.e. water that exits the bottom of the soil profile) as recharge to the grid cells of MODFLOW, and then pass MODFLOW-calculated groundwater-surface water fluxes to the stream channels of SWAT (Bailey et al., 2016). With this method, SWAT computes the volume of overland flow and soil lateral flow to streams, MODFLOW calculates the volume of groundwater discharge to streams, and then SWAT routes the water through channel networks of the watershed. Surface-groundwater interaction is simulated using river package of MODFLOW, with Darcy's law applied to calculate the volumetric flow of water through the cross-sectional flow area between the aquifer and stream channel (Bailey et al., 2016):

$$
Q_{\text {leak }}=k_{\text {bed }}\left(L_{s t r} P_{s t r}\right)\left(\frac{h_{s t r}-h_{g w}}{z_{\text {bed }}}\right)
$$


where:

$k_{b e d}$ is river bed hydraulic conductivity (L/T);

$L_{s t r}$ is the length of the stream (L);

$P_{s t r}$ is the wetted perimeter of the stream (L);

$h_{s t r}$ is river stage (L);

$h_{g w}$ is the hydraulic head of groundwater (L);

$z_{\text {bed }}$ is the thickness of the river bed (L); and

$Q_{\text {leak }}$ is negative if groundwater flows to the river (i.e. groundwater hydraulic head $h_{g w}$ is above the river stage $h_{s t r}$ ), and positive if river water seeps into the aquifer.

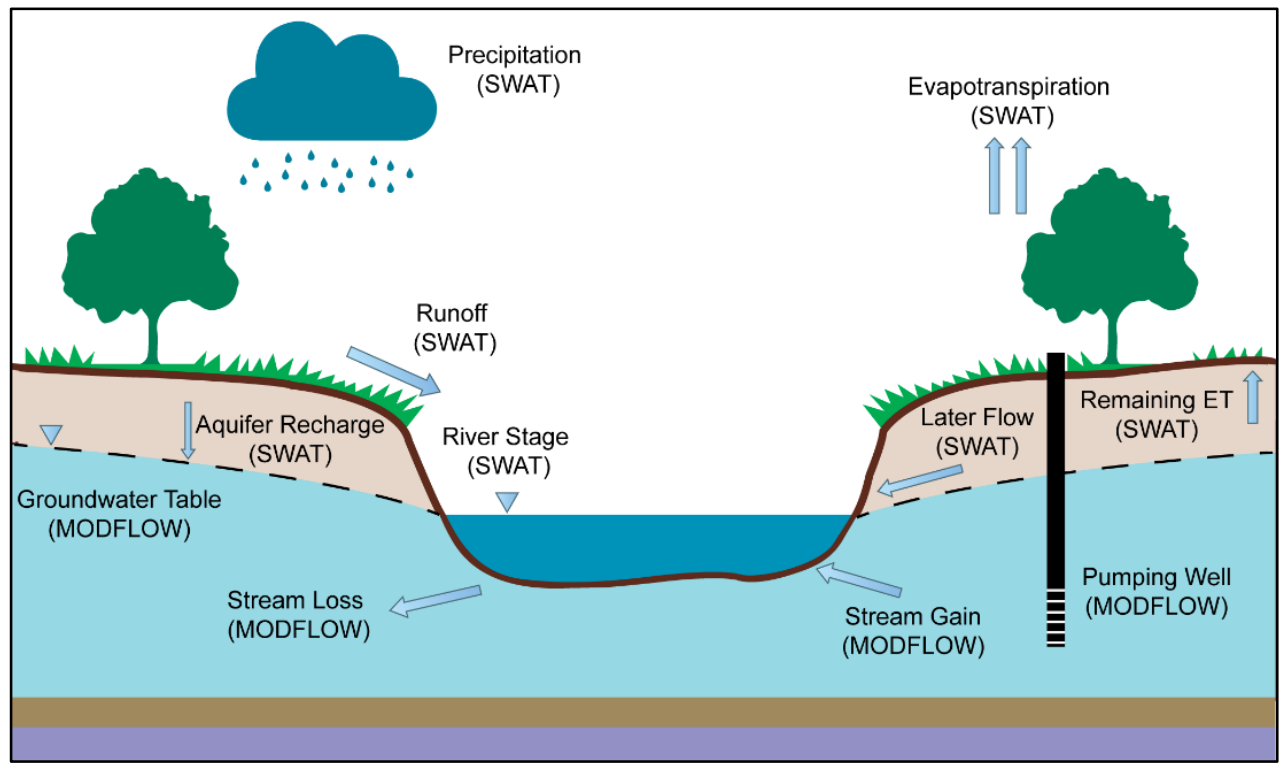

Fig. 6-2 Schematic representation of conceptual water balance of coupling SWAT-

\section{MODFLOW}

Data are passed between the models using 'mapping' subroutines that relates HRUs to MODFLOW grid cells and MODFLOW river cells to SWAT stream channels (Bailey et al., 2016). The main elements of this mapping scheme are: HRUs; Disaggregated HRUs (DHRUs), which divide each original HRU into individual, contiguous areas within a sub-basin allow HRU calculations to be geo-located; MODFLOW grid cells; MODFLOW River cells; and SWAT stream channels. The calculated deep percolation (i.e. recharge) for HRUs are first mapped to each individual DHRU, and 
then mapped to each MODFLOW grid cell according to the per cent of an area of the DHRU contained within the grid cell for use by the recharge package. SWATcalculated channel depth from each sub-basin is mapped to the group of River cells within the sub-basin for use by the River package (Bailey et al., 2016). Figure 6-2 reveals the schematic representation water balance of SWAT-MODFLOW model.

MODFLLOW then computes groundwater hydraulic head and groundwater-surface water interactions, which are passed to SWAT. Groundwater discharge volumes, calculated on a cell by cell basis within MODFLOW, are summed and added to instream flow for each SWAT sub-basin.

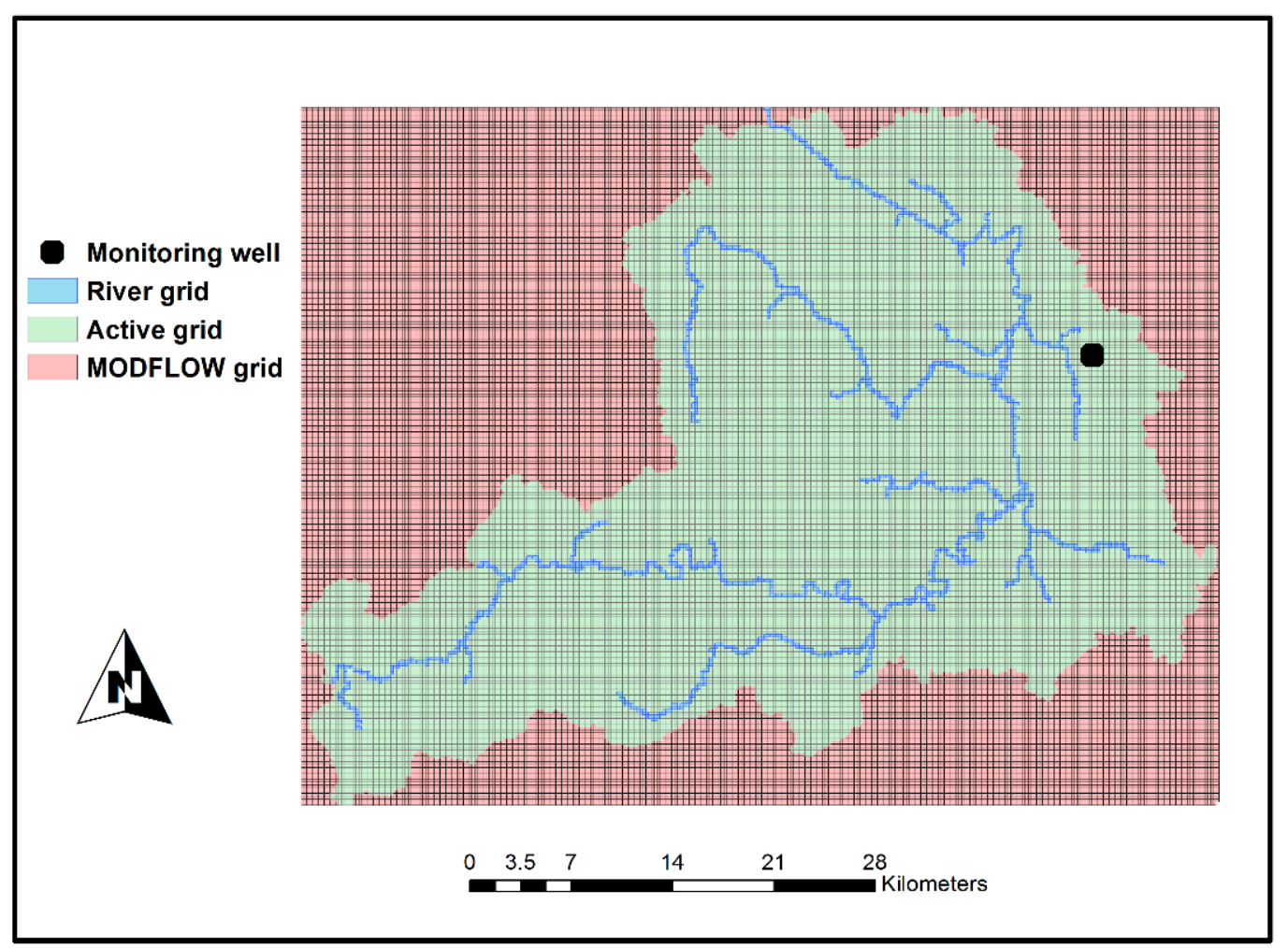

Fig. 6-3 MODFLOW grid with the location of groundwater monitoring well in the Dee River basin

SWAT then completes the stream routing calculations for the day, with the daily loop continuing until the end of the simulation. For the possible scenario of River cell 
intersecting more than one stream, the length of each stream within the cell is used to calculate the composite weighted value of channel depth for use by MODFLOW and to distribute the cell groundwater discharge volume to associated sub-basin main channels. Within this scheme, MODFLOW is called as a subroutine within the SWAT framework, providing a single compiled FORTRAN code (Bailey et al., 2016). Figure 6-3 shows the location of the groundwater monitoring well (at the east of the watershed).

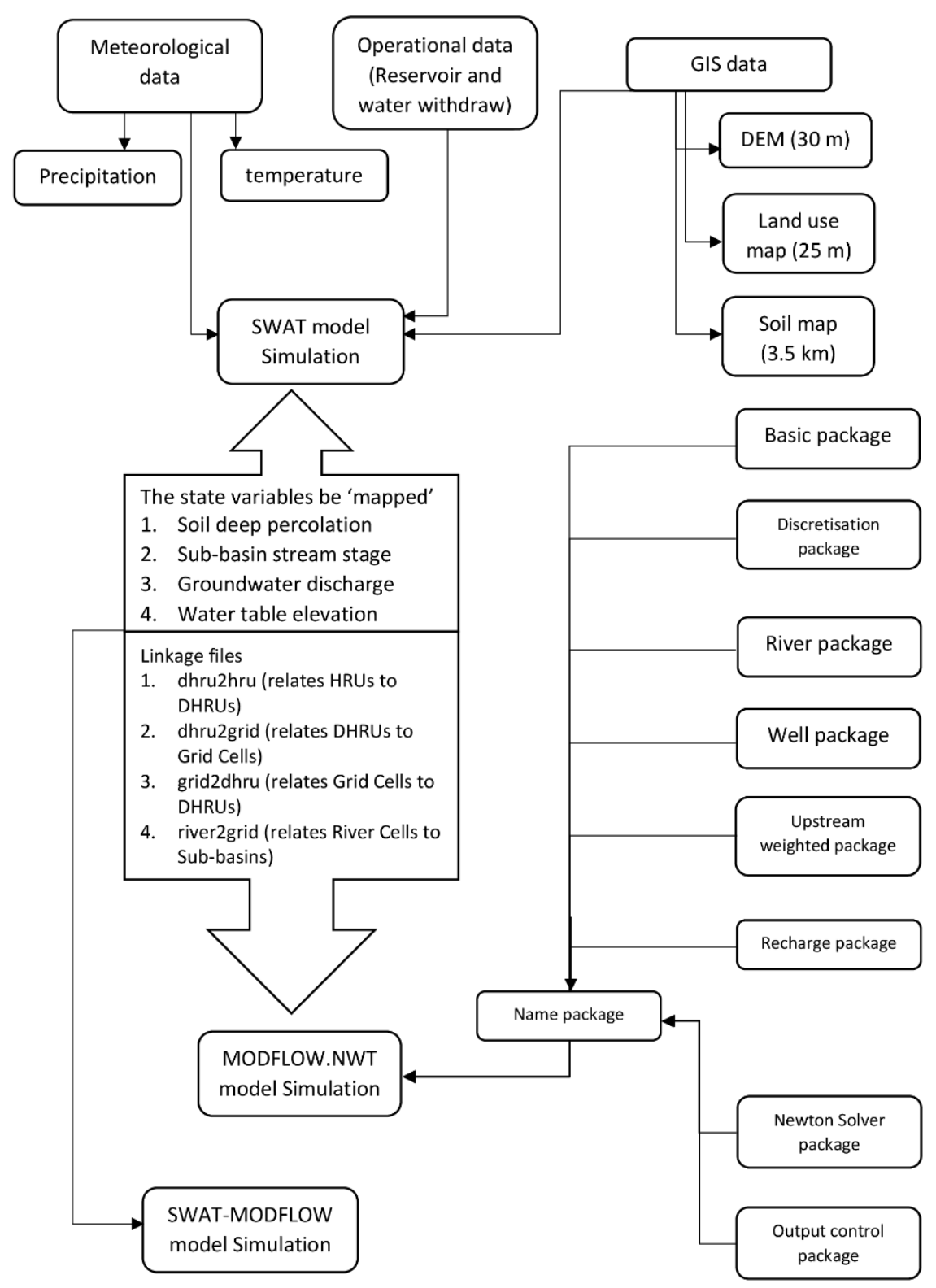

Fig. 6-4 Flowchart illustrating the coupled SWAT-MODFLOW model for Dee River watershed 
Figure 6-4 presents the process of coupling SWAT-MODFLOW models. The SWATMODFLOW model simulation and linking processes are illustrated in Fig. 6-5. The simulation runs through the repeated daily process of SWAT HRU calculations, passing data to MODFLOW, running MODFLOW, passing data to SWAT and routing water through the watershed's stream network upon reading input data for both the SWAT and MODFLOW models.

\section{Read Inputs}

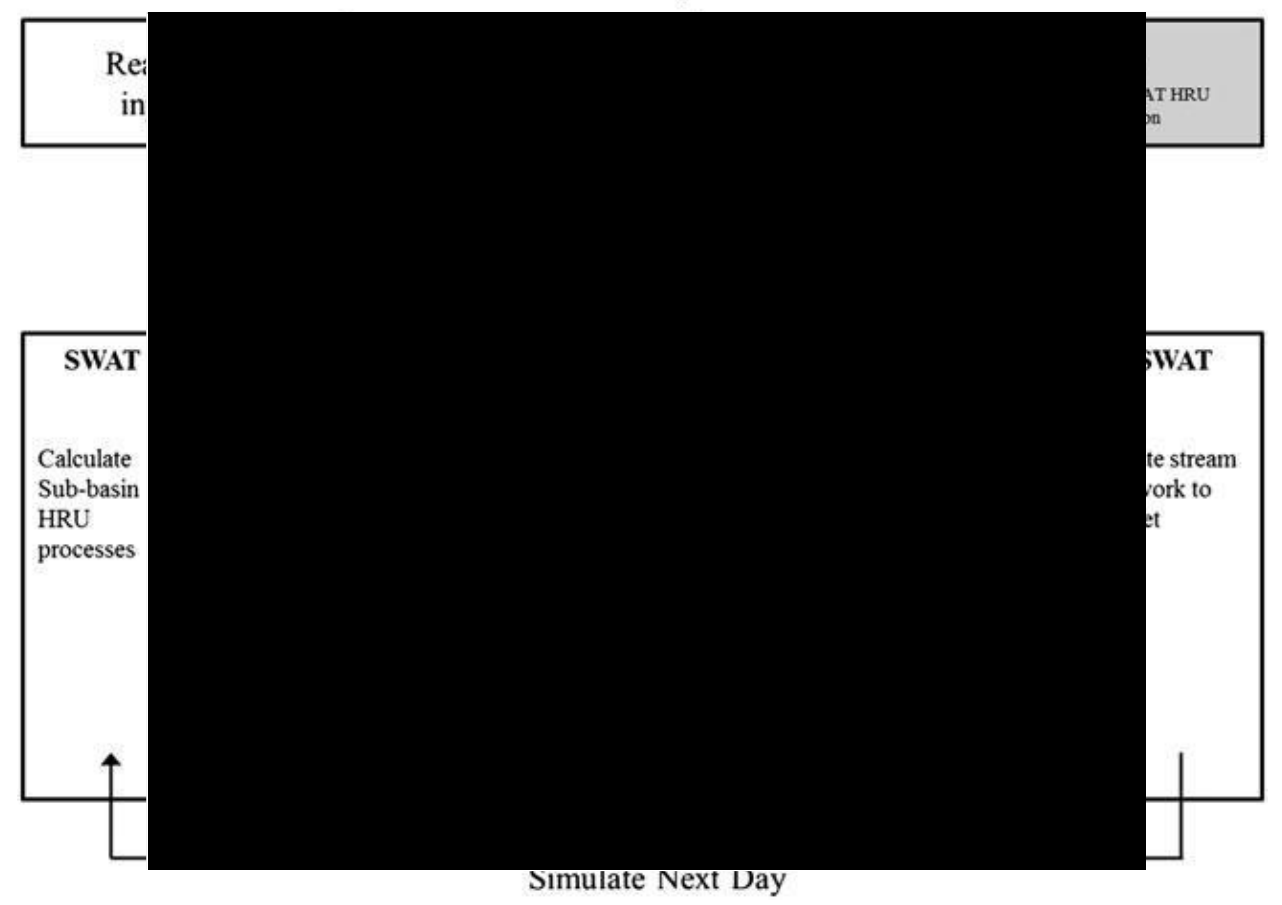

Fig. 6-5 Flowchart presenting the model code sequence of the coupled SWATMODFLOW model (After Bailey et al., 2016)

\subsubsection{Baseflow separation}

This baseflow separation procedure is based on a recursive digital filter commonly used in signal analysis and processing (Lyne and Hollick, 1979). It was used by Nathan and McMahon (1990) among others. In fact, this technique is arbitrary and physically unrealistic. Nevertheless, it does provide a subjective and repeatable estimate of baseflow that is easily automated (Nathan and McMahon, 1990). The filter given by Lyne and Hollick (1979) is expressed as: 


$$
Q_{s f, i}=\lambda Q_{s f, i-1}+\frac{1+\lambda}{2}\left(Q_{s u r f, i}-Q_{s u r f, i-1}\right)
$$

where $Q_{s f, i}$ is the direct runoff on $i^{\text {th }}$ day, $Q_{s u r f}$ is the surface runoff, and $\lambda$ is the filter parameter. Baseflow is calculated as below:

$$
Q_{b, i}=Q_{s u r f, i}-Q_{s f, i}
$$

where $Q_{b}$ is the baseflow. Baseflow separation is conducted using $\mathrm{R}$ statistical package 'EcoHydRology' (Fuka et al., 2015) to separate baseflow from the daily streamflow records.

\subsection{Results and discussion}

As mentioned before(section 6.2.2.), the calibrated daily SWAT model for Dee River watershed from chapter three is utilised here from 1992 to 2003 with three years' warm-up period, 1995-2000 calibration period and 2001-2003 as validation period. The boundary of the catchment of will be used as active cells region within the MODFLOW model. MODFLOW model using MODFLOW-NWT version is created for the study area using cell sizes of (200 x 200) m with a single layer, single stress period.

The standalone MODFLOW is manually calibrated by adjusting:

1) The horizontal permeability coefficient from the upstream-weighted package (to control the recharge rate); and

2) The river conductance from the river package (to control the surfacegroundwater interaction between the river channel and shallow aquifer).

Then, the model is coupled with the SWAT model to evaluate the simulated streamflow six river gauge station (Fig. 3-8, page 50) and the simulated groundwater head at the monitoring well (Fig. 6-3, page 135). 
Table 6-2 shows the simulated streamflow of the models for the calibration period, e.g., a standalone calibrated SWAT model and the coupled SWAT-MODFLOW model. Several indices are used including Nash-Sutcliffe Coefficient (NSE), R2 and percentage of bias (PBAIS) to measure the deviation of simulations from the observations at the chosen gauge stations. A slight decrease of NSE and R2 can be seen across all gauge stations except two stations where groundwater is dominant. Regarding PBIAS, the coupled model performs better or similar except that of the gauge Brynkinalt Weir.

Table 6-2: The calibrated river flow of the standalone SWAT model and the coupled SWAT-MODFLOW model for the calibration period of 1995-2000

\begin{tabular}{l|c|c|c|c|c|c}
\hline \multirow{2}{*}{ Station } & \multicolumn{3}{|c|}{ SWAT } & \multicolumn{3}{c}{ SWAT-MODFLOW } \\
\cline { 2 - 7 } & NSE & $\mathbf{R}^{\mathbf{2}}$ & PBIAS & NSE & $\mathbf{R}^{\mathbf{2}}$ & PBIAS \\
\hline Manley Hall & 0.94 & 0.98 & -5.80 & 0.90 & 0.98 & 14.50 \\
\hline Chester Ironbridge & 0.82 & 0.82 & -6.20 & 0.76 & 0.79 & 11.40 \\
\hline Suspension Bridge & 0.78 & 0.80 & -10.20 & 0.83 & 0.91 & 16.10 \\
\hline Pont-y-Capel & 0.80 & 0.82 & -14.70 & 0.77 & 0.78 & 8.80 \\
\hline Bowling Bank & 0.66 & 0.71 & -25.10 & 0.67 & 0.67 & -3.00 \\
\hline Brynkinalt Weir & 0.66 & 0.70 & 10.90 & 0.57 & 0.64 & 27.00 \\
\hline
\end{tabular}

Meanwhile, for the validation period (Table 6-3), the overall water balances (PBIAS) are improved for the coupled SWAT-MODFLOW are improved at three sites. The overall trends $\left(\mathrm{R}^{2}\right)$ are also enhanced as it is demonstrated in Table 6-3.

Table 6-3: The calibrated river flow of the standalone SWAT model and the coupled SWAT-MODFLOW model for the validation period of 2001-2003

\begin{tabular}{l|c|c|c|c|c|c}
\hline \multirow{2}{*}{ Station } & \multicolumn{3}{|c|}{ SWAT } & \multicolumn{3}{c}{ SWAT-MODFLOW } \\
\cline { 2 - 7 } & NSE & $\mathbf{R}^{\mathbf{2}}$ & PBIAS & NSE & $\mathbf{R}^{\mathbf{2}}$ & PBIAS \\
\hline Manley Hall & 0.92 & 0.94 & -3.20 & 0.88 & 0.98 & 16.30 \\
\hline Chester Ironbridge & 0.80 & 0.80 & -6.30 & 0.76 & 0.81 & 9.10 \\
\hline Suspension Bridge & 0.72 & 0.75 & -18.90 & 0.84 & 0.87 & 5.80 \\
\hline Pont-y-Capel & 0.67 & 0.76 & -21.00 & 0.68 & 0.68 & -1.80 \\
\hline Bowling Bank & 0.48 & 0.52 & -19.20 & 0.47 & 0.47 & -0.50 \\
\hline Brynkinalt Weir & 0.68 & 0.72 & 8.70 & 0.57 & 0.66 & 24.30 \\
\hline
\end{tabular}


The simulations from the standalone SWAT model and the coupled model are compared with the observed flow data at the river gauges. Figure 6-5 shows such comparison for the two selected stations (Chester Ironbridge) over the water year 1999. A remarkable feature revealed by Fig. 6-6 is that the coupled model outperforms the standalone SWAT model for the low flow conditions, particularly for the recessing curves parts of each peak. While both models simulate peak flow well, the standalone SWAT model does better for the 2nd peak. It is plausible that the MODFLOW component has well compensated the deficiency of SWAT in low flow representation (such as baseflow) in terms of taking more water as the recharge.

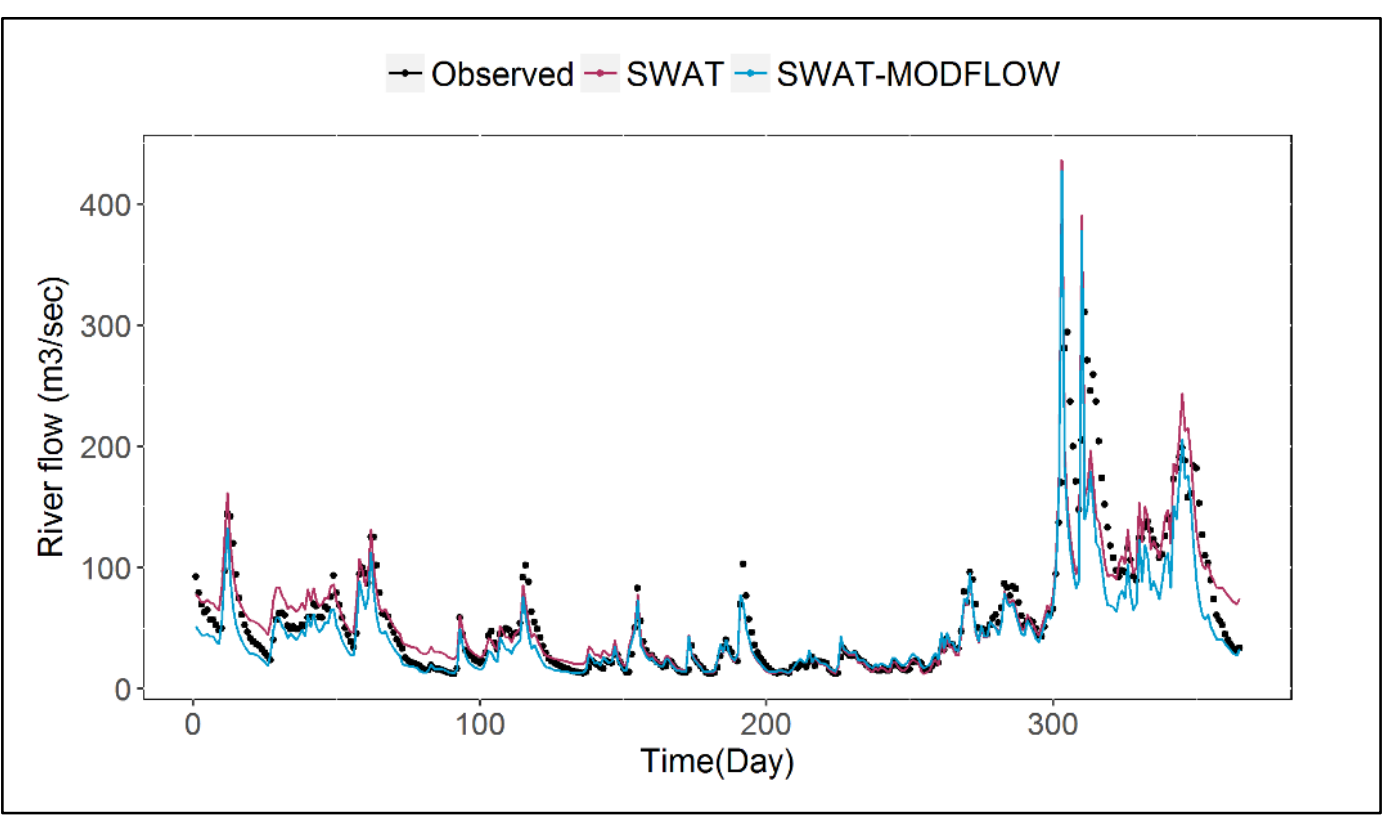

Fig. 6-6 The comparison of simulated river flow from the standalone SWAT model and the coupled SWAT-MODFLOW at Ironbridge for the water year of 1999

This is, in fact, an influential aspect of the coupled model, as it is more stressful in the flow period for water supply and the coupled model might be preferred in this occasions for better simulations. 
Table 6-4: The simulated baseflow results of the standalone SWAT model and the coupled SWAT-MODFLOW model for the calibration period of 1995-2000

\begin{tabular}{l|c|c|c|c}
\hline \multirow{2}{*}{ Station } & \multicolumn{2}{|c|}{ SWAT } & \multicolumn{2}{c}{ SWAT-MODFLOW } \\
\cline { 2 - 5 } & NSE & $\mathbf{R}^{\mathbf{2}}$ & NSE & $\mathbf{R}^{\mathbf{2}}$ \\
\hline Manley Hall & 0.76 & 0.90 & 0.88 & 0.96 \\
\hline Chester Ironbridge & 0.70 & 0.86 & 0.83 & 0.98 \\
\hline Suspension Bridge & 0.56 & 0.79 & 0.98 & 0.91 \\
\hline Pont-y-Capel & 0.57 & 0.87 & 0.67 & 0.85 \\
\hline Bowling Bank & -0.42 & 0.86 & 0.77 & 0.85 \\
\hline Brynkinalt Weir & 0.82 & 0.89 & 0.27 & 0.87 \\
\hline
\end{tabular}

A baseflow simulation of SWAT and coupled SWAT-MODFLOW are studied and presented. The NSE and R2 are employed to evaluate the baseflow simulation against observed one. Tables 6-4 and 6-5 show that SWAT-MODFLOW simulation has a better baseflow simulation than the standalone SWAT model.

Table 6-5: The simulated baseflow results of the standalone SWAT model and the coupled SWAT-MODFLOW model for the validation period of 2001-2003

\begin{tabular}{l|c|c|c|c}
\hline \multirow{2}{*}{ Station } & \multicolumn{2}{|c|}{ SWAT } & \multicolumn{2}{c}{ SWAT-MODFLOW } \\
\cline { 2 - 5 } & NSE & $\mathbf{R}^{\mathbf{2}}$ & NSE & $\mathbf{R}^{\mathbf{2}}$ \\
\hline Manley Hall & 0.55 & 0.79 & 0.79 & 0.94 \\
\hline Chester Ironbridge & 0.31 & 0.69 & 0.76 & 0.91 \\
\hline Suspension Bridge & -0.10 & 0.63 & 0.90 & 0.98 \\
\hline Pont-y-Capel & 0.27 & 0.89 & 0.58 & 0.74 \\
\hline Bowling Bank & -0.26 & 0.91 & 0.76 & 0.80 \\
\hline Brynkinalt Weir & 0.80 & 0.88 & 0.04 & 0.75 \\
\hline
\end{tabular}

Figure 6-7 reveals the baseflow from SWAT, SWAT-MODFLOW and the observed one at Pont-y-Capel station for the period of 1995-2000. Noticeably, SWATMODFLOW improves the origin of SWAT simulation regarding baseflow. 


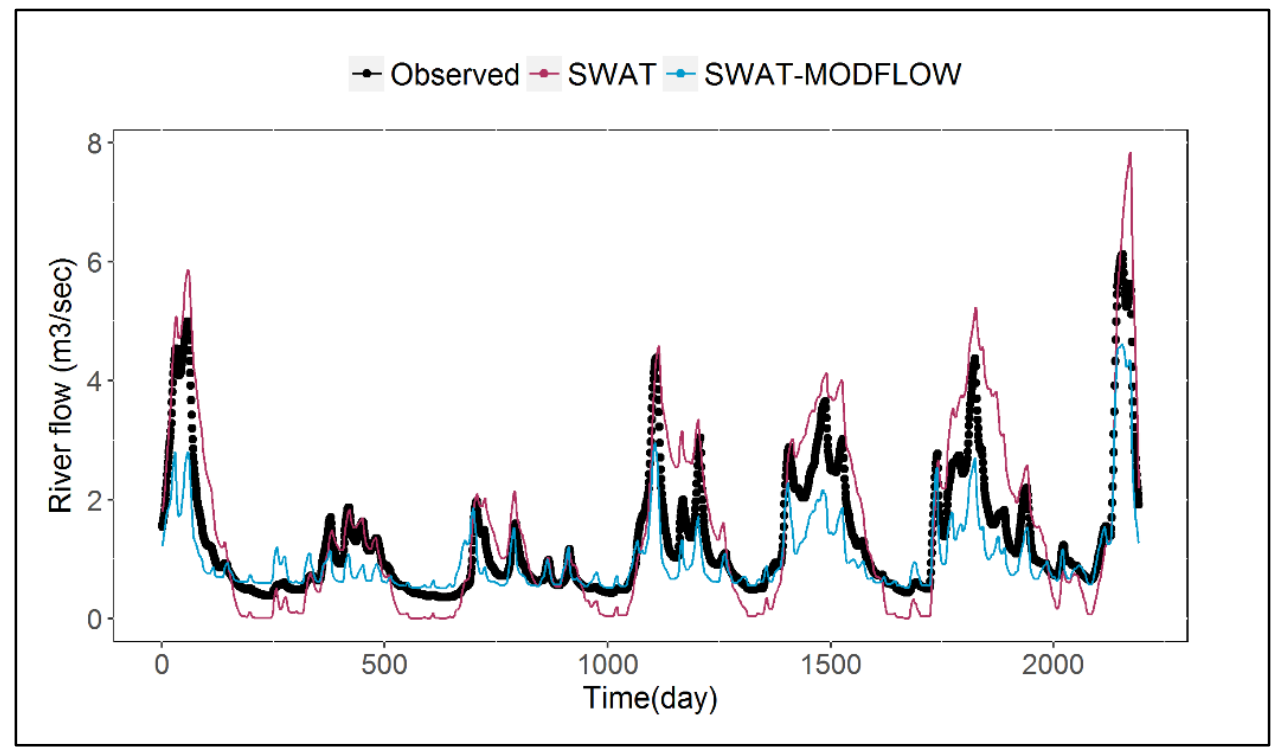

Fig. 6-7 The comparison of simulated baseflow from the standalone SWAT model and the coupled SWAT-MODFLOW at Pont-y-Capel for the period of 1995-2000

Figure 6-8 shows the comparison of daily simulated groundwater level against observed one at the monitoring well in the east of Dee watershed which shows that coupled SWAT-MODFLOW performs well with R2 of 0.87 for the calibration period of 1995-2000 and 0.88 for the validation period of 2001-2003.

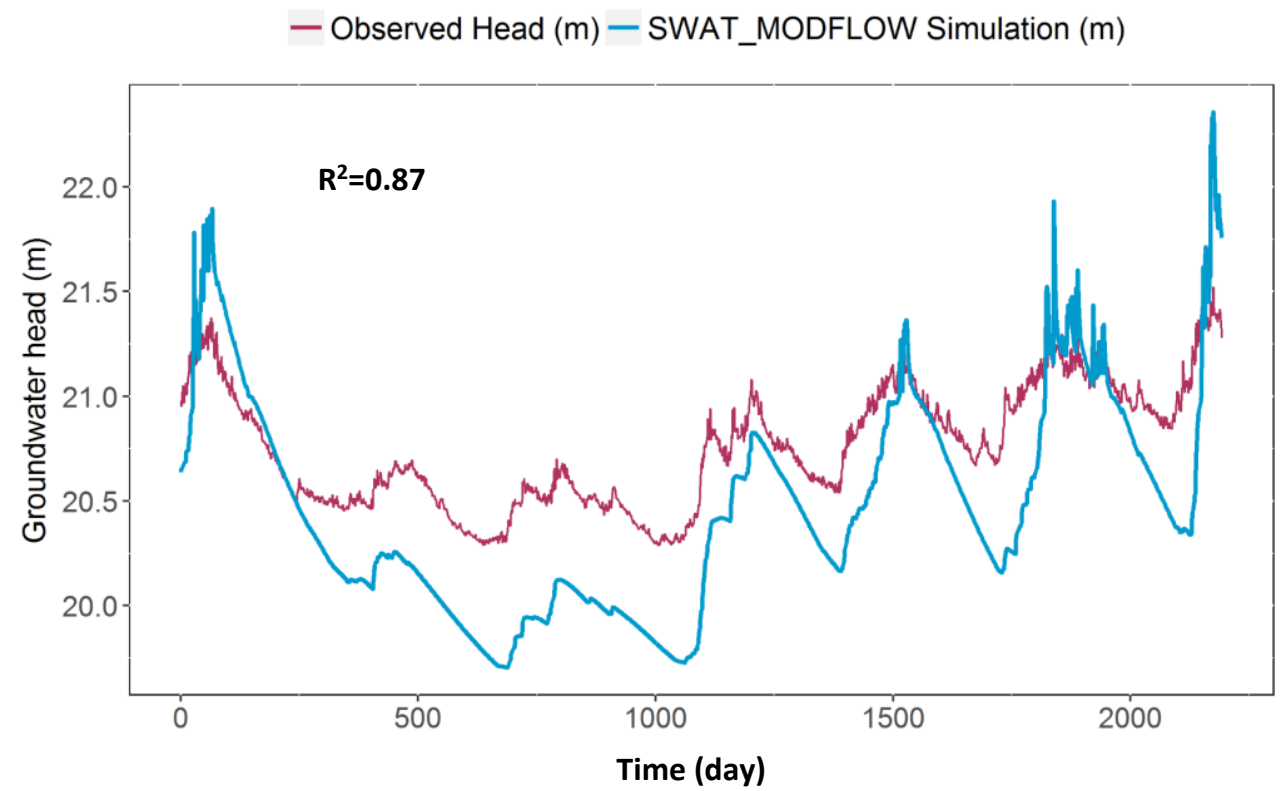

Fig. 6-8 The comparison of simulated groundwater level from the coupled SWATMODFLOW at the monitoring well for the period of 1995-2000 


\subsection{Summary}

In this chapter, the SWAT model that previously created in chapter 3 is coupled with groundwater flow model MODFLOW to simulate streamflow and baseflow for the Dee River basin. Baseflow, one of the key source of the streamflow and could be the primary source in the dry season, and that concern the decision makers of water resources management. It can be characterised by its hydrograph which is derived from the total streamflow hydrograph by different baseflow separation methods. While the performance of baseflow simulation of the SWAT model has been tested in many river basins, the primary objective of this chapter is to improve the simulated baseflow of the SWAT standalone by coupled surface-groundwater models (SWATMODFLOW).

The recursive digital filter technique is used for baseflow separation of observed and simulated river flow of the SWAT and coupled the SWAT-MODFLOW. The results show that the coupled model can produce comparably better simulations of baseflow in the stream network, and thereby improved the water balance in the catchment. Further work with the model will include additional calibration to improve stream flow and also groundwater level fluctuations. Overall, the study shows a promising direction for using coupled surface-groundwater model in IWRM. However, this might increase the parameters uncertainty of the complex model systems (coupled models) and computationally more expensive than the standalone model. 


\section{Chapter 7: Uncertainty Analysis of the Coupled Surface- Groundwater Models}

Hydrological models are often carried out for the reliable estimation of streamflow from upland areas into the downstream reach of river, reservoirs and lakes within a watershed and evaluation of the efficiency of various management practices. To serve such purposes, the application and selection of a suitable model become obligatory. The use of most hydrological models frequently requires a large number of spatially distributed variable input data and parameters. Attributable to the lack of higher quality of input data and the simplification of environmental processes, these models prerequisite to being calibrated by certain degrees to the observed hydrological variables such as river flow observation.

The practical quantification of prediction uncertainty of hydrological processes is valuable for the water resources planning and management and relevant decisionmaking processes (Liu and Gupta, 2007). The model predictions are uncertain values and have to be represented with a confidence range owing to uncertainties associated with the model input, model structure, parameter and model output (Van Griensven and Meixner, 2007). The model calibration technique is a rigorous and challenging process and influenced by the model complexity, the number of input parameters and iterations (Vanrolleghem et al., 2003). Model calibration and validation have been assessed through conducting uncertainty analysis (UA) and sensitivity analysis (SA) (Blasone et al., 2008).

In this chapter, a MATLAB toolbox, the Sensitivity Analysis for Everybody SAFE, will be used to run uncertainty and sensitivity analysis for the standalone SWAT model and coupled SWAT-MODFLOW for the Alyn River Basin (a branch in Dee River Watershed). The primary objective of this chapter is the analysis of parameters uncertainty of semi-distributed model (SWAT) and the coupled SWAT-MODFLOW which have not studied yet. 


\subsection{Overview}

Water resources management problems comprise complex processes from the subsurface and surface level to their interface regimes (Srivastava et al., 2013b). Moreover, water resources management problems are very challenging processes because hydrogeological characteristics within the catchment system are, in nature, heterogeneous with respect to both space and time (Strayer et al., 2003). The semi/fully distributed hydrological models are handy tools in water resources management (Patel and Srivastava, 2014), especially in assessing the impacts of climate change of land use on water resources within the catchment (Srivastava et al., 2013a). However, these kinds of models have a considerable amount of uncertainties among parameters estimation.

The evaluation of parameter uncertainty has gained attractiveness in sciences, including hydrological sciences (Yatheendradas et al., 2008). The calibration of watershed models is a challenging process because of (Tung, 2005):

1) Natural randomness: uncertainty in nature (the real world) is caused by inherent randomness in natural processes. Additionally, it is difficult to eliminate because it needs a complete understanding of natural systems under study;

2) Model uncertainties: this is as a result of the conceptual simplification of natural processes of the model and owing to some processes that might not be considered by the model;

3) Parameter uncertainties: it is the inability of a model to precisely evaluate input variables and model parameters which is attributable to the lack of sufficient data and the inherent inconsistency of model inputs in time and space;

4) Data uncertainties: this uncertainty arises as a result of errors in data handling, measurement and limitation of data in time and space, which results in an insufficient representation of the study area; and

5) Operational uncertainties: these uncertainties are associated with maintenance and human errors, construction deterioration and manufacture. 
SWAT model has been broadly used in many countries worldwide for streamflow prediction and for soil and water conservation (Patel and Srivastava, 2013). Sensitivity Analysis (SA) and Uncertainty Analysis (UA) are necessary processes to decrease uncertainties of models obliged as a result of the variation of model structure and parameters (Gupta et al., 2006). Presently, many techniques have been developed for the calibration and uncertainty analysis techniques for hydrological models such as:

1) Sequential Uncertainty Fitting, SUFI 2 (Abbaspour et al., 2007);

2) Generalized Likelihood Uncertainty Estimation, GLUE (Beven and Binley, 1992);

3) Parameter Solution, ParaSol (Yang et al., 2008);

4) Markov Chain Monte Carlo, MCMC (Vrugt et al., 2008); and

5) Particle Swarm Optimization, PSO (Eberhart and Kennedy, 1995).

These methods have been linked to the SWAT model through SWAT-CUP software (Abbaspour et al., 2007) and facilitate UA and SA of model structure and parameters (Rostamian et al., 2008).

Currently, the SWAT model is widely used in hydrological modelling by the scientific community. Most of the studies on SWAT dedicated on the calibration and validation of SWAT for surface runoff such as (Chu and Shirmohammadi, 2004; Ahl et al., 2008; Baker and Miller, 2013). Some researchers such as Heuvelmans et al. (2006), Shen et al. (2012) and Mishra (2009) have quantified the uncertainty associated with hydrological modelling and sensitivity analysis using various optimization algorithm, a couple of other researchers such (Yang et al., 2008; Xue et al., 2014) performed modelling studies focusing on the uncertainty analysis using SWAT model.

In this chapter, a MATLAB toolbox, the Sensitivity Analysis for Everybody (SAFE), will be used for analysing the uncertainty in the modelling results for SWAT model and Coupled SWAT-MODFLOW for the Alyn River Basin (branch in Dee River Catchment). The analysis is performed based on daily streamflow flow for eight years (1993-2000) including two years warm-up period. The objectives of this chapter are: 
1) Perform parameters uncertainty analysis for streamflow simulation using techniques embedded in the SAFE toolbox for both the SWAT model and the coupled SWAT-MODFLOW; and

2) Demonstrate the applicability and feasibility of these techniques for analysing uncertainties in streamflow simulation.

\subsection{Study area}

The Alyn River is one of the tributaries of the Dee River Catchment located in the north region of the catchment with total area $222 \mathrm{~km}^{2}$ as revealed in Fig. 7-1. It rises from the southern end of the Clwydian Hills and the Alyn Valley passing through Mold city before reaching its convergence with the Dee River to the northeast of Wrexham city.

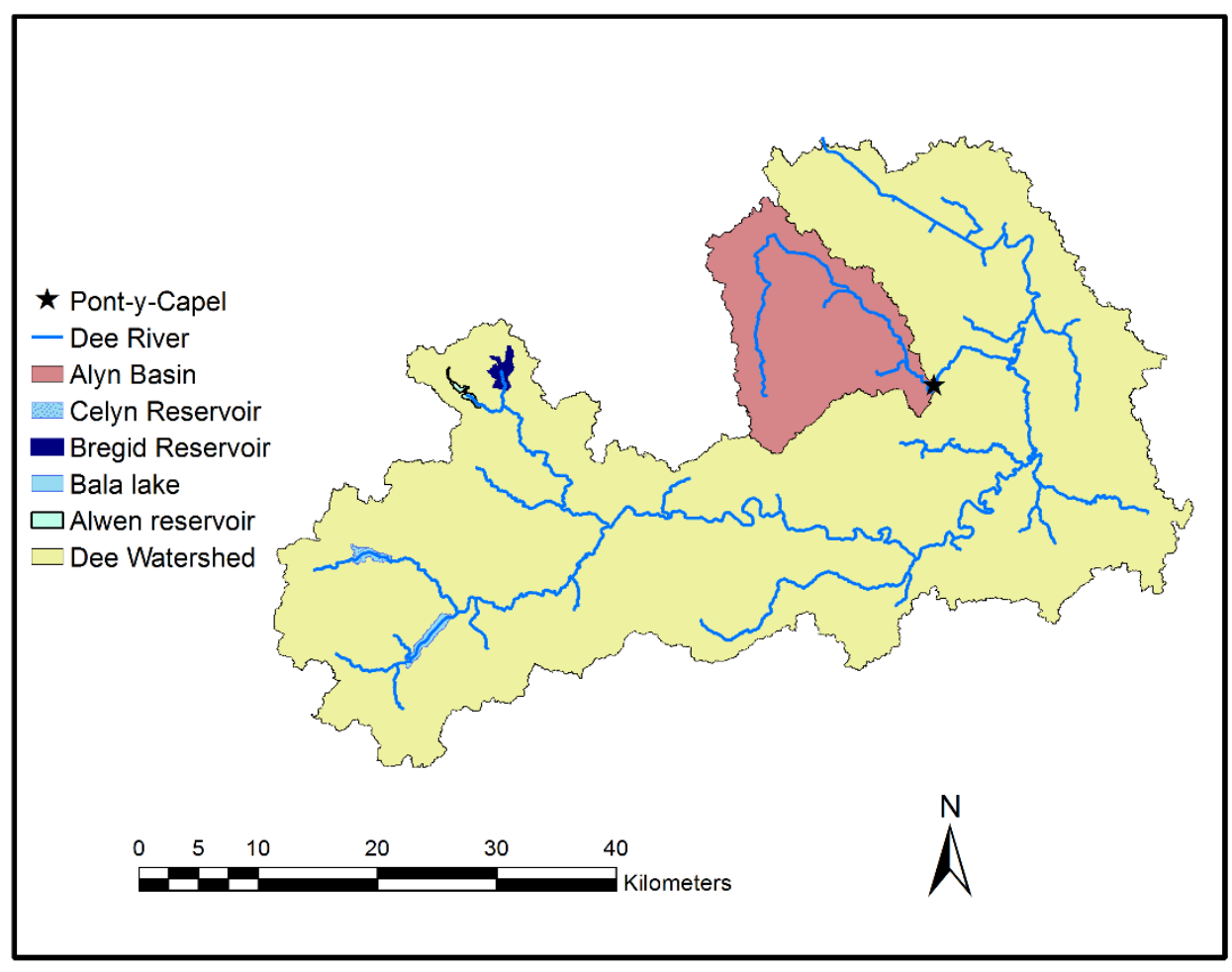

Fig. 7-1 Location of Alyn River Catchment

\subsection{Sensitivity Analysis}

Global Sensitivity Analysis GSA is a term defining a set of mathematical methods to examine how the deviation in the output of a numerical model can be endorsed to 
variations of model inputs (Pianosi et al., 2015). Several GSA techniques have been suggested and used in the literature and their application in the environmental modelling domain has increased gradually in recent years (Yang, 2011; Tang et al., 2007; Pianosi et al., 2015).

GSA has been acknowledged as a vital tool for the assessment and development of environmental models (Saltelli et al., 2008). In this chapter, the MATLAB toolbox for the application of GSA, called SAFE (Sensitivity Analysis for Everybody) (Pianosi et al., 2015), will be used to perform uncertainty and sensitivity analysis for SWAT and coupled SWAT-MODFLOW models.

The SAFE Toolbox has mainly been considered to make GSA accessible to nonspecialist users (i.e. people with a basic knowledge of MATLAB and/or GSA). It is also created to allow more skilled users to feasibly further develop the code (Pianosi et al., 2015). The SAFE Toolbox contains several GSA methods comprises:

1) Variance-Based Sensitivity Analysis, VBSA(Saltelli et al., 2008);

2) Regional Sensitivity Analysis, RSA (Wagener and Kollat, 2007);

3) The Fourier Amplitude Sensitivity Test, FAST (Cukier et al., 1973);

4) The Elementary Effects Test, EET (Morris, 1991);

5) Dynamic Identifiability Analysis, DYNIA (Wagener et al., 2003); and

6) A Novel Density-based Sensitivity technique, PAWN (Pianosi and Wagener, 2015); and

7) Generalized Likelihood Uncertainty Estimation, GLUE (Beven and Binley, 1992).

This Toolbox also provides some visualisation tools comprising (Pianosi et al., 2015):

1) Scatter (dotty) plots; and

2) The parallel coordinate plot and the visual test for validation of screening suggested by Andres (1997).

SA is an analytical tool that can lead the model calibration and validation and support the prioritisation of efforts for uncertainty reduction (Norton, 2015; Song et al., 2015). 
Generally, such purposes are applied as four different purposes of GSA (Saltelli et al., 2008; Sarrazin et al., 2016):

1) Screening (or Factor Fixing): which refers to the identification of those input factors, if any, which have no influence on the model output and hence can be fixed to any value within their feasible range with negligible implications on the output. For example, in Vanuytrecht et al. (2014), screening of model parameters is carried out as an initial stage to inform a successive calibration, which is tailored to the subset of influential parameters;

2) Ranking (or Factor Prioritization): which defines the ordering of the input factors according to their relative effect on the model output. It is classically utilised to increase the understanding of the model and to recognise main controls of the model's behaviour (e.g. Van Werkhoven et al., 2008), and to prioritize efforts for the reduction of uncertainty (Sin et al., 2011), or to support development of models (Hartmann et al., 2013);

3) Variance Cutting: that is employed for the reduction of the variance of the output to a value below a user-chosen tolerance. It targets at finding specific sensitivities for the various input factors and is, for instance, conducted in risk assessment and reliability (e.g. Saltelli and Tarantola, 2002); and

4) Factor Mapping: aims to recognise those conditions (e.g. sub-ranges of input factors such as forcing inputs or parameters) that produce critical values of the output. It can be utilised to support robust decision-making or to enhance understanding of a model (Singh et al., 2014).

\subsection{Global Sensitivity Analysis (GSA) techniques}

In this section, it is intended to introduce two GSA methods that will be used in this study, Elementary Effect Test (Morris Method) and Generalised Likelihood Uncertainty Estimation GLUE method.

\subsubsection{Elementary Effect Test (EET)}

The Elementary Effect Test EET (Morris, 1991) is a more suitable method when dealing with time-consuming models (Saltelli et al., 2008). EET technique is a global 
extension of One-factor-At-the-Time (OAT) Local SA methods. It is based on the computation of several Elementary Effects (EEs). Explicitly, the EE of the $i^{\text {th }}$ input factor $\mathrm{x}_{\mathrm{i}}$ at given baseline point $X^{j}$ and for a predefined perturbation $\Delta$ is given by:

$$
\begin{array}{r}
E E_{i}^{j}=\frac{y\left(x_{1}^{j}, x_{2}^{j}, \ldots, x_{i-1}^{j}, x_{i}^{j}+\Delta, \ldots x_{M}^{j}\right)}{\Delta} \\
-\frac{y\left(x_{1}^{j}, x_{2}^{j}, \ldots, x_{i-1}^{j}, x_{i}^{j}, \ldots x_{M}^{j}\right)}{\Delta}
\end{array}
$$

For each input factor, EEs are calculated at $n$ randomly selected baseline points across the input factor space. The estimated mean $\left(\mu_{i}\right)$ of the EEs is chosen as a measurement of the total effects of the $i^{t h}$ input factor. The standard deviation $\left(\sigma_{\mathrm{i}}\right)$ of the EEs can be deduced as the intensity of the interactions of the $i^{\text {th }}$ input factor with other input factors. To avoid compensations between EEs of opposite sign, the mean of the absolute values of the EEs $\left(\mu_{\mathrm{i}}{ }^{*}\right)$ will be used in this study, as first suggested by Campolongo et al. (2007):

$$
\mu_{i}^{*}=\frac{1}{n} \sum_{j=1}^{n}\left|E E_{i}^{j}\right|
$$

The sensitivity index of Eq.7-2 offers a semi-quantitative measurement of sensitivity, principally suitable to rank the factors on an interval scale (Saltelli et al., 2008). To define baseline points and the perturbation $\Delta$, the radial design strategy proposed by Campolongo et al. (2011) is implemented as it was revealed that radial based design is computationally capable. In this method, $\mathrm{n}$ baseline points are sampled across the input factor space, and associated with other $\mathrm{n}$ auxiliary points, are also selected randomly.

Then, the perturbation $\Delta$ is calculated as the difference between the $\mathrm{i}^{\text {th }}$ coordinate of the auxiliary and baseline point. The baseline and auxiliary points were produced by Latin hypercube sampling to maximise the coverage of the input factor space. The 
total number of model evaluations required to compute the mean EEs for all input factors is equal to:

$$
N=n \cdot(M+1)
$$

It is noted that the value of $\mu_{i}^{*}$ has no specific meaning per se, since it depends on the scale and units of measurements of the model output y. Consequently, to allow for comparison between different case studies, it defines a normalised mean of the EEs as our sensitivity index, i.e. the ratio between $\mu_{i}^{*}$ and the maximum value of the mean EEs across all the input factors:

$$
S_{i}^{E E T}=\frac{\mu_{i}^{*}}{\max _{k} \mu_{k}^{*}}
$$

The sensitivity index of Eq.7-4 now takes values between 0 and 1 regardless of the units of measurement of $y$, and it expresses input factor sensitivity as a fraction of the sensitivity for the most significant input factor. The index still offers a semiquantitative measure of sensitivity.

\subsubsection{Generalised Likelihood Uncertainty Estimation (GLUE)}

The Generalised Likelihood Uncertainty Estimation (GLUE) method, which was introduced by Beven and Binley (1992), is an innovative uncertainty technique that is frequently used with environmental models simulation. GLUE is a favourite technique for the uncertainty quantification owing to its simplicity and applicability to nonlinear problems including those for which a unique calibration is not ostensible. Moreover, it is widely used because it has utilised in real-world applications and that it seems to provide the desired representation of uncertainty (Montanari, 2005). Blasone et al. (2008) reported that attribute to GLUE's conceptual simplicity, its flexibility with different sources of information and ease of implementation, it can be combined with various criteria to define a likelihood measurement. 
The basic concept of the GLUE is to integrate a prior knowledge of the model parameters captured by the prior PDF with new information reflected in the observed data as characterised by the likelihood measure to obtain a posterior PDF of the model parameters. Beven and Binley (1992) introduced their identifiable requirements on their likelihood measures arguing that "the choice of a likelihood measure will be inherently subjective."

In the GLUE method, parameters uncertainty account for all sources of uncertainty such as input uncertainty, structural uncertainty, parameter uncertainty and response uncertainty. As a result, this method has been broadly utilised in several areas as an effective and general strategy for model calibration, validation and uncertainty quantification associated with complex models.

The calculation of the likelihood of a particular set of parameters is the main feature of GLUE and is different from the traditional technique of calibration, validation and uncertainty quantification. Parameter sets that result in their likelihood values below a certain threshold are called 'non-behavioural' and are excluded. The remaining 'behavioural' parameter sets are assigned rescaled likelihood weights that sum to 1 and hence look like probabilities. According to Beven and Binley (1992), two conditions should be satisfied by the 'likelihood measurement':

1) "It should be zero for all simulations that are considered to exhibit behaviour dissimilar to the system under study."

2) "It should increase monotonically as the similarity in behaviour increases."

Beven and Binley (1992) argued that the likelihood function could be selected from "many of the goodness-of-fit indices used in the past." They also reported that the choice of likelihood function would be greatly affected the resulting uncertainty intervals and so argue that this selection must be made obvious so they can be the "subject of discussion and justification" (Beven and Freer, 2001). Various likelihood measurements have been presented and used with GLUE previous applications. The method of inverse error variance, a popular likelihood measurement, which was familiarised and introduced by Beven and Binley (1992): 


$$
L_{I V}=\left[s_{\varepsilon}^{2}\right]^{-N}
$$

where $s_{\varepsilon}$ is the standard deviation of the model errors, $\mathrm{N}$ is the shaping factor by Beven and Freer (2001). Beven and Binley (1992) used N=1 but suggested that the shaping factor can be also selected by the modeller. The different values of $\mathrm{N}>0$ lead to different descriptions of uncertainty (Ratto et al., 2001). The increasing $\mathrm{N}$ provides a greater weight to model parameters which yield a better goodness of fit. As $\mathrm{N}$ reaches infinity the best parameter set that is generated will be given a weight of 1 , whereas all other parameter sets will be discarded. As $\mathrm{N}$ approaches zero, all parameter sets receive equal weight.

The likelihood measurement adopted frequently employs Nash Sutcliffe efficiency index as follow:

$$
L_{N S E}=\left[1-\frac{s_{\varepsilon}^{2}}{s_{Q}^{2}}\right]^{N}
$$

where $\mathrm{S}_{\varepsilon}$ is the standard deviation of the errors, $\mathrm{S}_{\mathrm{Q}}$ is the standard deviation of the observations and again, $\mathrm{N}$ is a shaping parameter. Examples applications of GLUE with this efficiency index can be found in Uhlenbrook and Sieber (2005). This index only makes sense if in the calculation of $s_{\varepsilon}^{2}$ assumes that the errors have zero mean. Otherwise, the systematic bias would be neglected.

The likelihood function will be used to evaluate simulated streamflow by SWAT and SWAT-MODFLOW against observed values. In this chapter, the Nash-Sutcliffe efficiency index (NSE) is selected because it is the most often used likelihood measurement for GLUE based on the literature (Beven and Freer, 2001). The NSE value ranges from $-\infty$ to 1 , with 1 representing a perfect fit. Uniform distribution is selected owing to its simplicity and the lack of a prior distribution of a parameter. The 
drawback of a typical GLUE method is its expensive computational burden imposed by its random sampling strategy. Hence in this study, an improved sampling method was introduced by combing Latin hypercube sampling with GLUE. Therefore, a large number of sampling sets (10,000 times) for SWAT model and (1,000 times) for SWAT-MODFLOW were conducted.

In this chapter, the standalone SWAT and the coupled SWAT-MODFLOW models are evaluated with different parameter sample sets for the prediction of daily river flow. Sensitivity indexes, as well as the convergence of them, are then analysed. The uncertainty bands based on the GLUE analysis are then produced and compared for both the standalone model as well as the coupled model.

\subsection{Results and discussion}

\subsubsection{Uncertainty of parameters}

The SWAT model for the Alyn River basin is previously created, calibrated and validated based on daily observed river flow (refers to chapter 3, section 3-5) at Ponty-Capel river gauge station (see Table 3-5 in chapter 3). The SWAT model is thoroughly calibrated and validated using the SUFI2 algorithm. Meanwhile, a groundwater flow model is constructed using MODFLOW.NWT version (refers to chapter 6 , section 6.2.3). A total number of 15 parameters are used in the calibration of SWAT (Table 3-6 in chapter 3) and 2 parameters in MODFLOW. Then, calibrated models are coupled.

In this chapter, parameters uncertainty is investigated for the most sensitive parameters of the SWAT standalone and the coupled SWAT-MODFLOW over the period of 1995-2000 using the SAFE toolbox as illustrated in Table 7-1. The runs are designed to make for every parameter (or group HRU for the same parameters) separately and to create dotty plots for the SWAT and the coupled SWATMODFLOW. Then parameters are grouped into a single run for both SWAT standalone and coupling SWAT-MODFLOW and combined the run with Elementary 
Effect Test (EET) and Generalized Likelihood Uncertainty Estimation (GLUE) methods for further investigation of parameters uncertainty. The combination of parameters as well as the rate of change is demonstrated in Table 7-2.

Table 7-1: SWAT and SWAT-MODFLOW model parameters

\begin{tabular}{llll}
\hline Model & Parameters & Mode of change & Range of change \\
\hline & CN2.mgt & Relative & $-25 \%$ to $+25 \%$ \\
\hline & ESCO.hru & Relative & $-25 \%$ to $+25 \%$ \\
\hline & CN2.mgt & Relative & $-25 \%$ to $+25 \%$ \\
& k.upw & Relative & $-25 \%$ to $+25 \%$ \\
& Conductance.riv & Relative & $-25 \%$ to $+25 \%$
\end{tabular}

Parameters uncertainty are examined over the 82 HRUs of SWAT model and the distributed groundwater parameters of MODFLOW such as the horizontal hydraulic conductivity and the river conductance. Figure 7-2 and 7-4 demonstrate the variation of the goodness of fit (i.e. NSE and PBIAS) for the Alyn River watershed as a function of variation of parameters considered in this study ( 2 parameters for the SWAT standalone and 3 for the coupled SWAT-MODFLOW respectively).

Table 7-2: Combined parameters for SWAT and coupling SWAT-MODFLOW

\begin{tabular}{llll}
\hline Model & Parameters & Mode of change & Range of change \\
\hline $\begin{array}{l}\text { CN2.mgt } \\
\text { (HRU74) }\end{array}$ & Relative & $-30 \%$ to $+50 \%$ \\
\hline $\begin{array}{l}\text { ESCO.hru } \\
\text { (HRU74) }\end{array}$ & Relative & $-75 \%$ to $+40 \%$ \\
& $\begin{array}{l}\text { CN2.mgt } \\
\text { (HRU74) }\end{array}$ & Relative & $-30 \%$ to $+50 \%$ \\
k.upw & Relative & $-50 \%$ to $+50 \%$ \\
& $\begin{array}{l}\text { ESCO.hru } \\
\text { (HRU74) }\end{array}$ & Relative & $-75 \%$ to $+40 \%$ \\
Conductance.riv & Relative & $-50 \%$ to $+50 \%$
\end{tabular}


By discerning scatter plots for SWAT model (Fig. 7-2), it is noticeable that the primary sources of streamflow uncertainty are due to 2 parameters curve number (CN2) and soil evaporation compensation factor (ESCO). Seemingly, CN2 and ESCO for HRU 74 (black regions in Fig. 7-3) are the most identifiable parameters for the study River basin as can be seen in Figs. 7-2b and 7-2d. This could be explained by the fact HRU 74 has a larger area within the study basin.

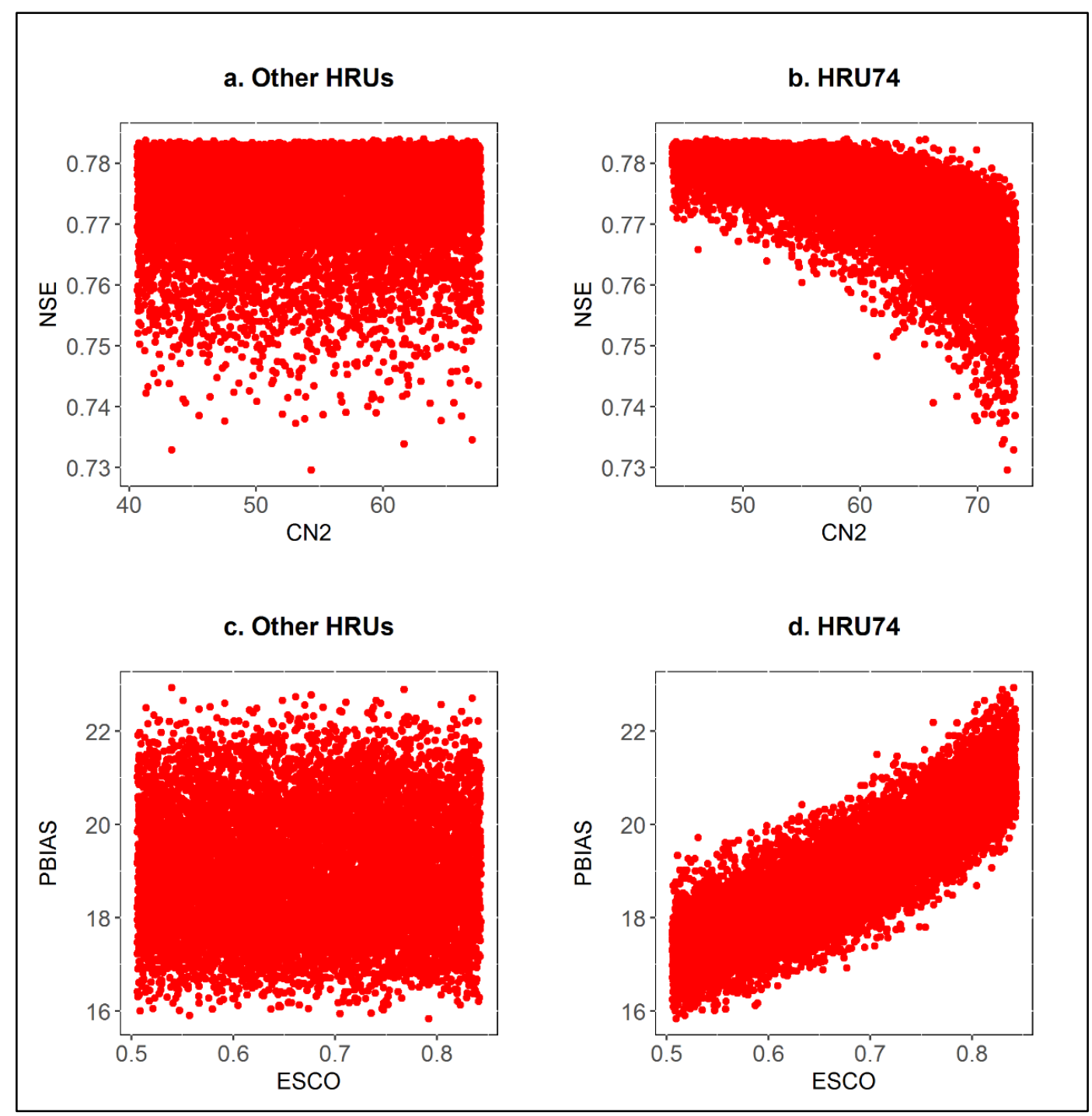

Fig. 7-2 The scatter plot map for streamflow simulation of SWAT model

For the other HRUs, the existence of multiple peaks in the Nash-Sutcliffe coefficient for $\mathrm{CN} 2$ and Percent of bias of ESCO indicated that estimation of these parameters might not be feasible as revealed in Figs. 7-2a and 7-2c. The coupled SWAT- 
MODFLOW shares the same features with respect to the CN2 parameters as from the standalone SWAT simulations, as demonstrated in Figs. 7-4a (other HRUs) and 7-4b (HRU 74).

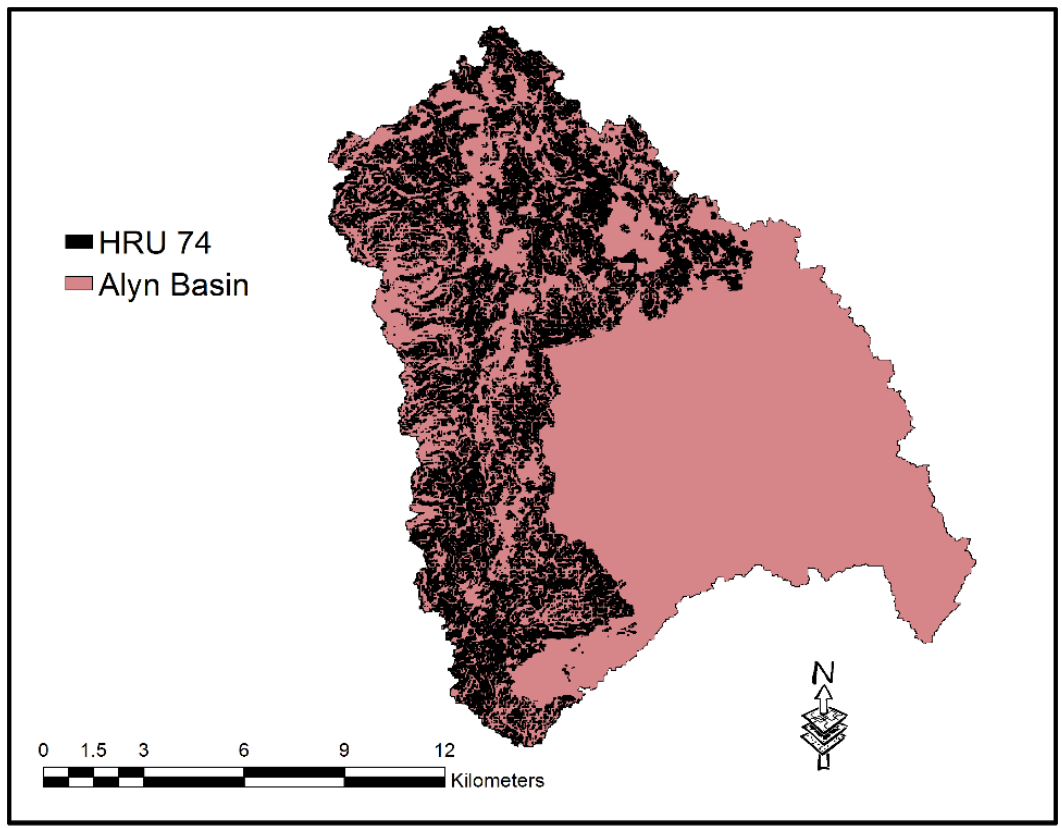

Fig. 7-3 Location of HRU 74 with Alyn River Basin

It is worthy to mention that non-identifiability of a parameter does not mean that the model is not sensitive to these parameters. Lenhart et al. (2002) point out that CN2 is deemed as the primary source of uncertainty while dealing with streamflow simulation. This study points out that for both the standalone SWAT model and the coupled SWAT-MODFLOW that CN2 demonstrated non-identifiability in the stream flow simulation. There are two similar studies carried out on the SWAT model only such as Kannan et al. (2007) and Shen et al. (2012). The likely reason would be that there is an explicit provision in the SWAT model to update the CN2 value for each day of simulation based on available soil moisture content (Shen et al., 2012). Accordingly, a change of $\mathrm{CN} 2$ will not significantly influence water balance components.

The estimation of non-identifiable parameters (CN2 for SWAT and coupled SWATMODFLOW) and ESCO for SWAT model for Alyn River basin will be difficult since there might be many combinations of these parameters that would produce a similar 
model performance. For the other parameters of coupled SWAT-MODFLOW, horizontal permeability (kMODFLOw) and river conductance are identifiable parameters in the study basin as can be seen in Figs. 7-4c, 7-4d, 7-4e and 7-4f. This could be explicated by the fact that $\mathrm{k}_{\text {MODFLOW }}$ and river conductance represented soil characteristics of the coupled SWAT-MODFLOW. Consequently, an increase in these parameters would cause an increase in the estimate of surface runoff and baseflow.

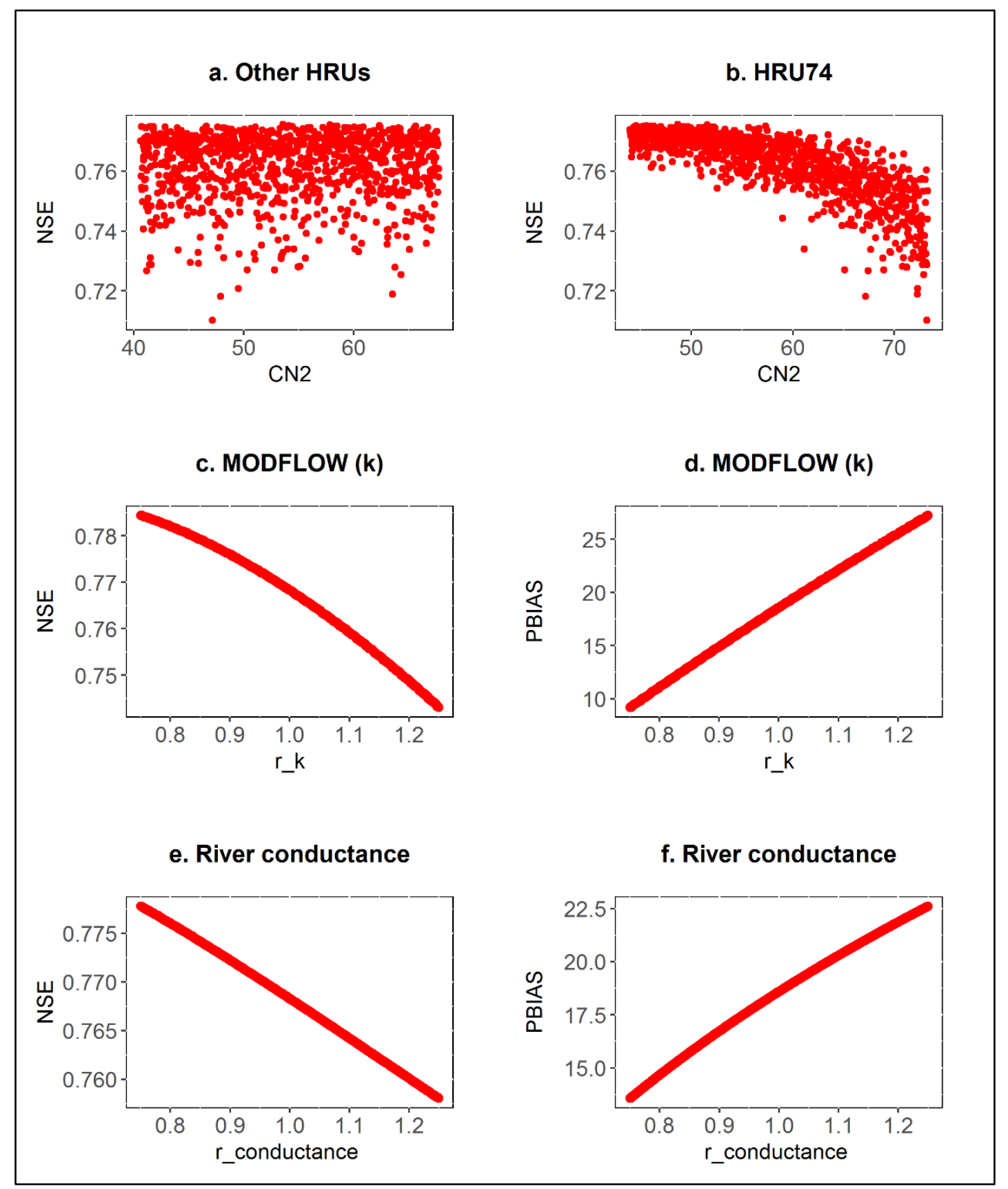

Fig. 7-4 The scatter plot map for streamflow simulation of the coupled SWATMODFLOW 


\subsubsection{Parameters uncertainty using the EET method}

In this section, results of the screening threshold and the convergence study are investigated. Figures 7-5 and 7-6 reveal for the average of Elementary Effects versus their standard deviation with confidence bounds and the evolution of the sensitivity indices increasing numbers of model evaluations.

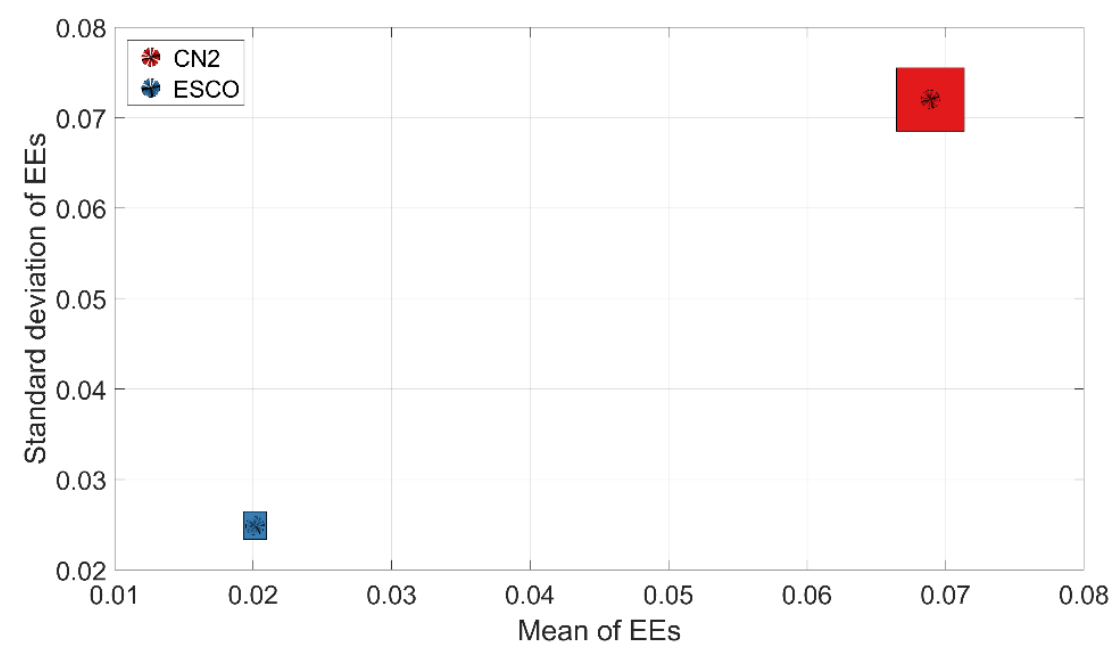

a. SWAT

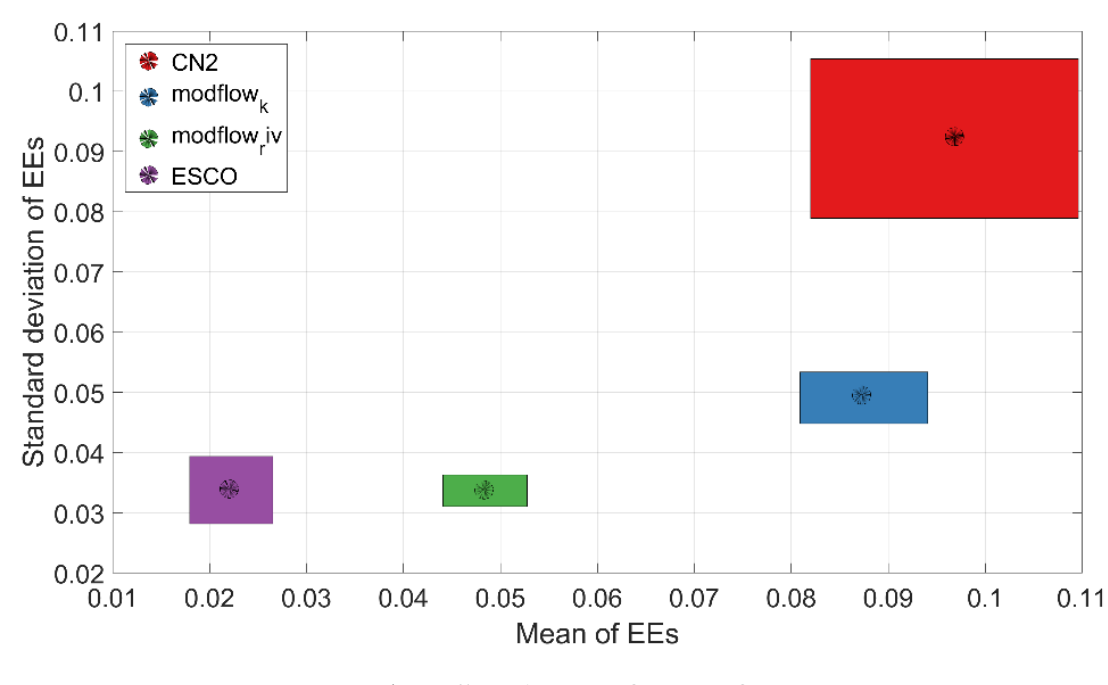

b. SWAT-MODFLOW

Fig. 7-5 Average of Elementary Effects against their standard deviation with confidence bounds from bootstrapping 
Figures 7-6 demonstrates the order of the most sensitive parameters using the EET method for the SWAT (Fig. 7-6a) and the coupled SWAT-MODFLOW (Fig. 7-6b). The ranking by this method identifies the most sensitive parameter for SWAT model is CN2 as revealed in Figs. 7-5a and 7-6a and ESCO is the less sensitive one. Meanwhile, for the coupled SWAT-MODFLOW, CN2 and MODFLOW $k$ (k.upw) are the most sensitive parameters as shown in Figs. $7-5 b$ and $7-6 b$ and the less sensitive are ESCO and MODFLOW riv (conductance.riv).

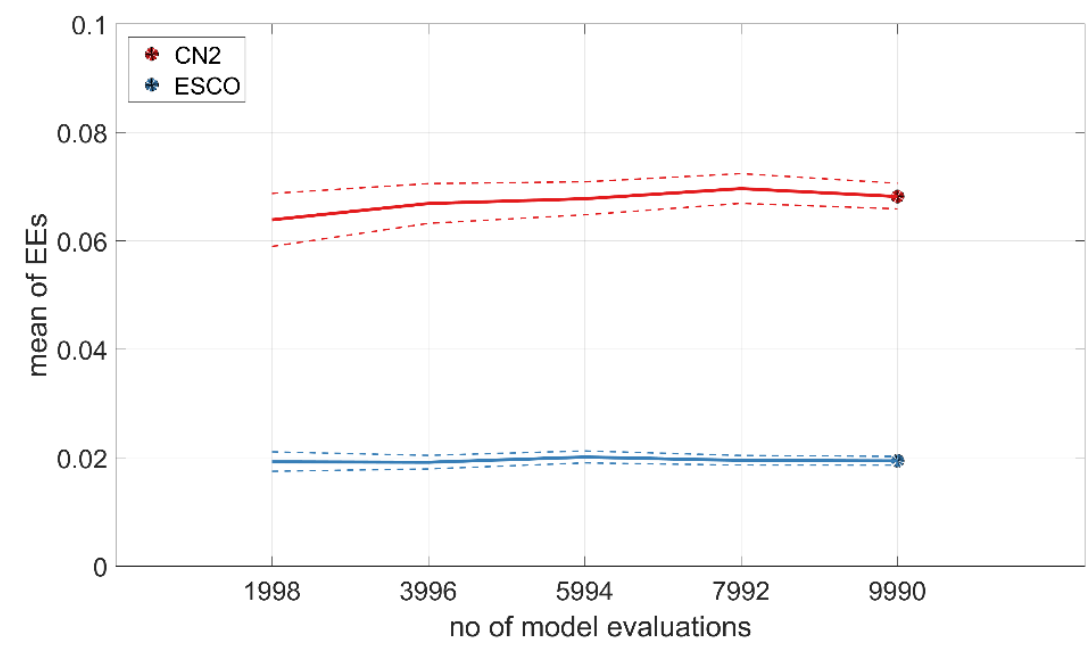

a. SWAT

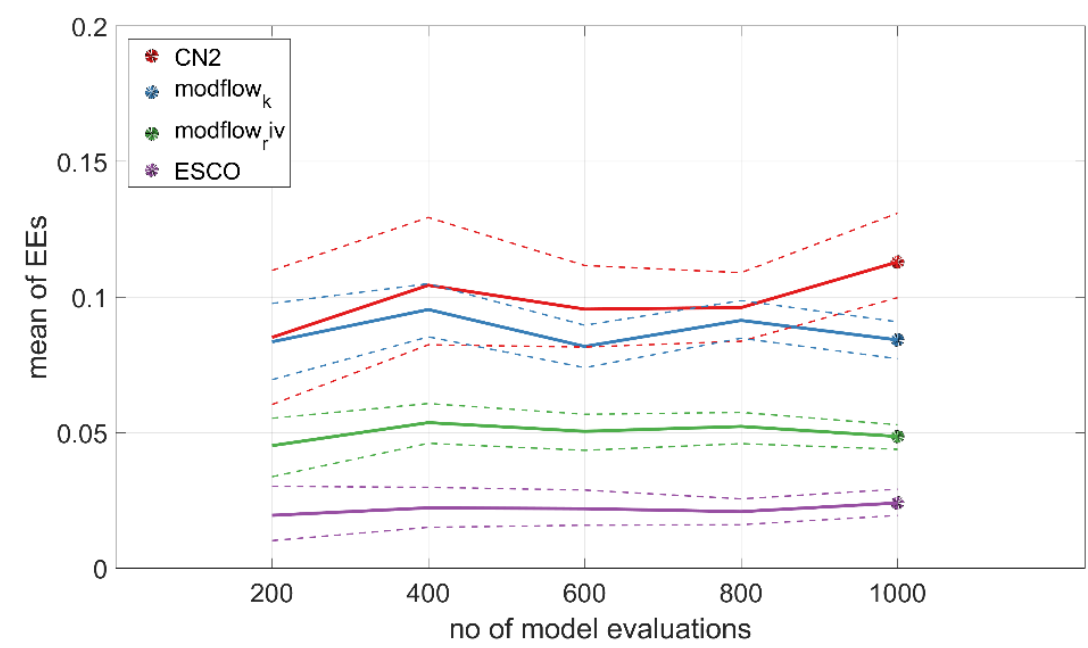

b. SWAT-MODFLOW

Fig. 7-6 Convergence plots of the sensitivity indices of the model parameters for the SWAT and the coupled SWAT-MODFLOW using Elementary Effect Test (EET). The solid lines are the bootstrap means of the sensitivity indices and the dashed lines are the $95 \%$ bootstrap confidence intervals. 
Noticeably, for SWAT-MODFLOW, the two most sensitive parameters (CN2 and MODFLOW $_{\mathrm{k}}$ ) separate out whereas other parameters have very close values of the sensitivity indices. Slight fluctuations in parameters that have a relatively high sensitivity index values can lead to substantial differences in ranking. The convergence rate for the ranking seems to be governed by the specific study area and on the relative importance of the sensitivity indices among the different parameters. For instance, the coupled SWAT-MODFLOW (Fig. 7-6b), the sensitivity indices of the two most dominant parameters are knowingly higher than others, whereas for the SWAT model (Fig. 7-6a) they are more evenly spread. Accordingly, the ranking of the most significant parameters stabilises faster for the coupling SWAT-MODFLOW than for SWAT standalone.

\subsubsection{Parameters uncertainty using GLUE method}

In this section, the SWAT model and the coupled SWAT-MODFLOW simulations with likelihood value below the threshold value of the parameters are considered to be $2 \mathrm{~m}^{3} / \mathrm{s}$. whereas, the likelihood value higher than the threshold value are normalised and sorted according to the river flow simulation. The selection range of the parameters for the SWAT standalone and the coupled SWAT-MODFLOW is subjectively defined in Table 7-2.

The scatter plots representing the behavioural responses of all parameters of SWAT and SWAT-MODFLOW as shown in Figure 7-7. The parameter distributions and the sharp peak can evaluate the sensitivity of parameters. Here, CN2 (SCS curve number) has revealed small variability in relation to the highest likelihood with clear peak subsequent into highly sensitive parameters for both the SWAT and the coupled SWAT-MODFLOW. In Alyn River basin, CN2 is the most sensitive parameter

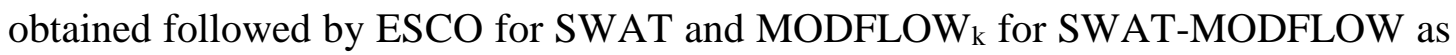
illustrated in Figs 7-7a (SWAT) and 7-7b (SWAT-MODFLOW). The parameters ESCO and MODFLOW riv in SWAT-MODFLOW are less capable of obtaining information due to the structural scantiness of the variable. 

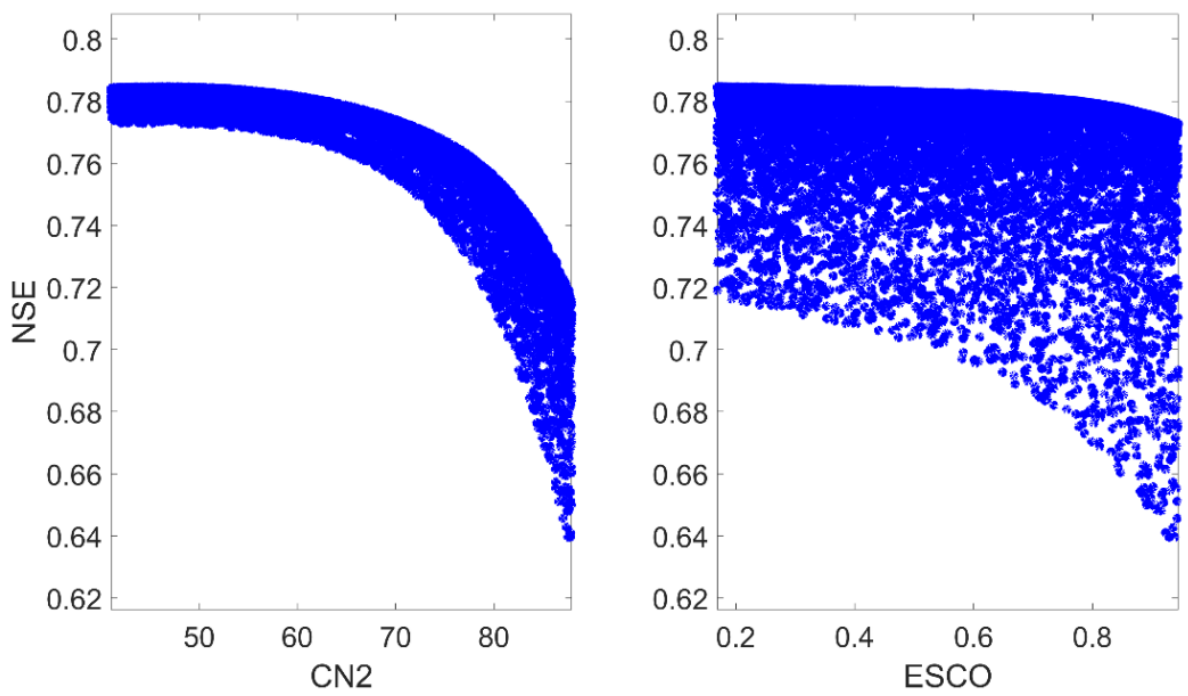

a. SWAT
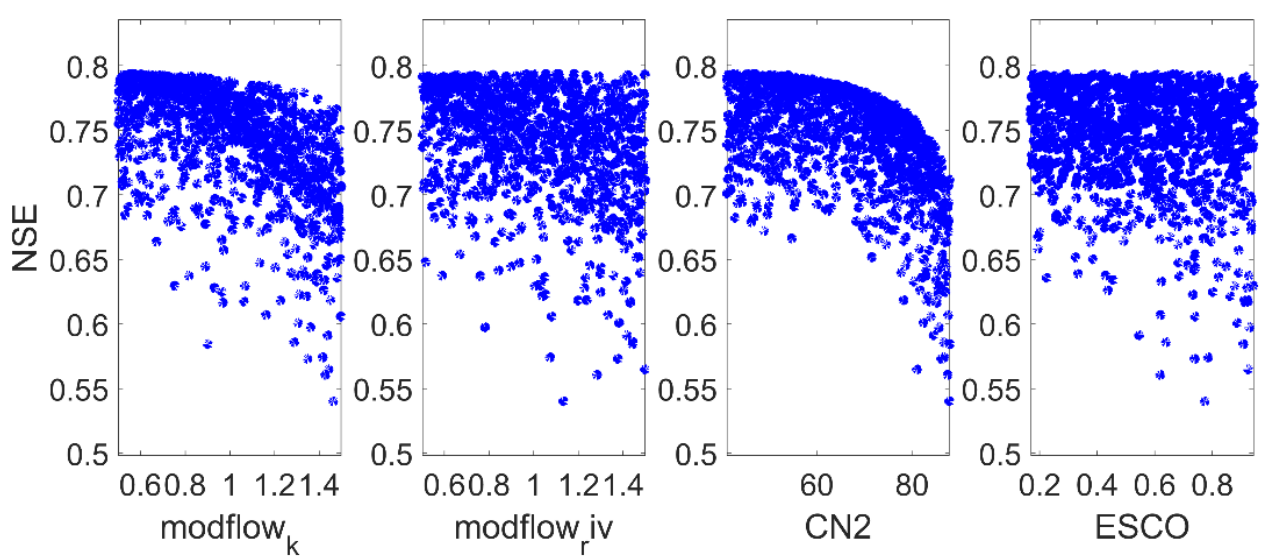

b. SWAT-MODFLOW

Fig. 7-7 Scatter plots of parameters for the SWAT and the coupled SWATMODFLOW using GLUE method

Simulated and measured streamflow for the water year of 1997 is shown in the plot for better understanding as in Fig. 7-8. The trend and peaks of the observed flow are agreeably followed by the simulated flow in the given plot. 


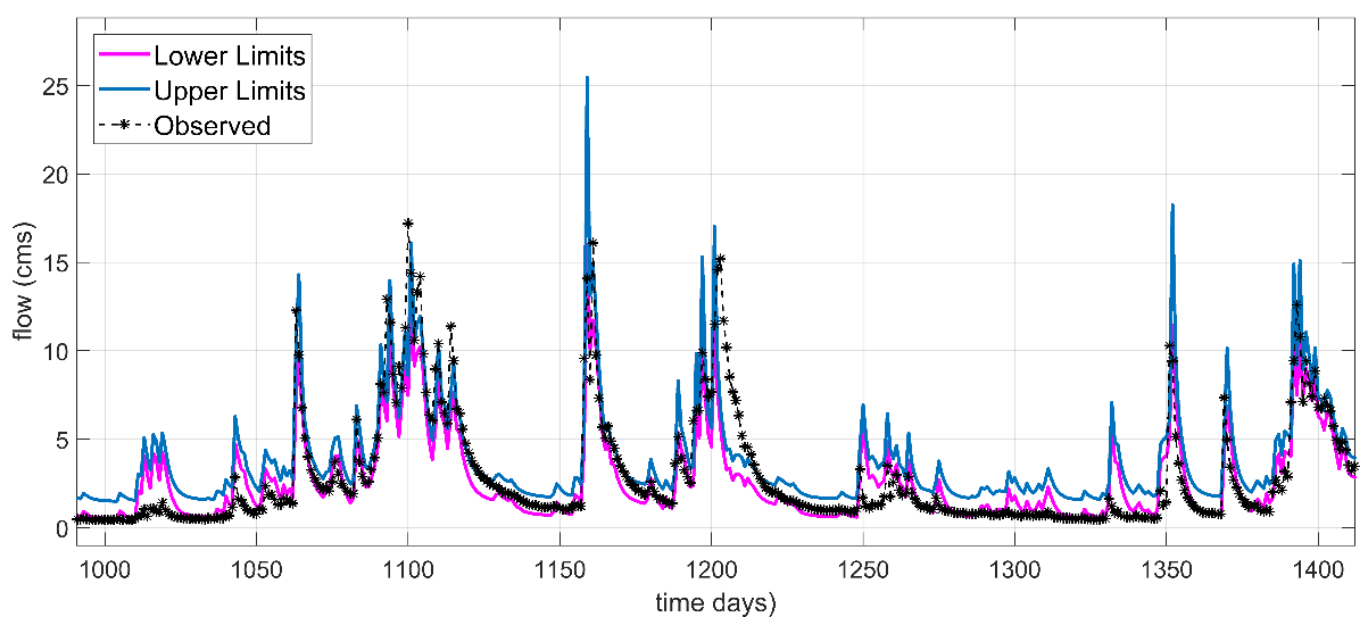

Fig. 7-8 Streamflow prediction limits against observations by GLUE method at Ponty-Capel for Alyn River basin for the water year of 1997

\subsection{Summary}

In this chapter, parameters uncertainty are studied for the most sensitive parameters for the SWAT and the coupled SWAT-MODFLOW for the period of 1995-2000 in Alyn River basin using the SAFE toolbox. The runs are created to make for every single parameter (or group HRU for the same parameter) separately and construct dotty plots for the SWAT standalone and the coupled SWAT-MODFLOW. Then parameters are grouped into a single run for both the SWAT and the coupled SWATMODFLOW and combined with Elementary Effect Test (Morris method) and GLUE methods for further analysis of parameters uncertainty. A total number of 10,000 simulations for SWAT model and 1,000 simulations with the coupled SWATMODFLOW.

Several important points can be concluded:

1) Model sensitivities and uncertainties towards the input parameters are the key base of model development and improvement which can solve the problems associated with water resources planning and management;

2) Since parameter uncertainty was only capable of quantifying a small part of whole uncertainty in the models, this study suggests further studies prerequisite on model structure. 
3) The term parameters 'equifinality' exhibited there is no unique parameter estimation for some parameters in this study, and therefore uncertainty in the appraised parameters in the study basin is recognisable. This result agreed well with some other studies as aforementioned previously.

4) The likely reason for 'equifinality' is because parameters obtained from the calibration process were influenced by some other causes such as sensitivity or insensitivity in parameters and correlations amongst parameters.

5) This chapter describes a recent effort to investigate parameters uncertainties for the integrated surface-groundwater model (SWAT-MODFLOW) on the hydrological modelling output. What distinguishes this study from others is it is the first time that modeller is able to run uncertainty analysis of the coupled (SWAT-MODFLOW) in a single platform.

6) This study also explores the feasibility of run parameters uncertainty of a large number of parameters (distributed-based of a single parameter). The change of the distributed value of parameters concurrently (i.e. all HRUs and/or grid) for the coupled SWAT-MODFLOW is a computationally intensive process, especially for a large river basin. This rise the need for a parallel computing or Highperformance computing HPC to adjust all parameters simultaneously with less computational time at a single platform.

Indeed, more studies are needed concerning the issues found in this study, which include but are not limited to:

1) Because of a long time of the coupled SWAT-MODFLOW, only 1,000 simulations is considered. Therefore, it is suggested to create more runs with 10,000 and larger;

2) In this study, only one ranking/screening method is used (EET method) to rank the most sensitive parameters. More technique of ranking and screening might utilise for the comparison; and

3) More parameters need to be combined and analysed with GLUE and with different screening methods 


\section{Chapter 8: The Reliability of Climate Projections in the Concept of Driving Models}

Study of climate change impact on hydrological events, such as floods and droughts for the water resource management, has become increasingly relying on the use of climate projections produced by climate models. One of the immediate concerns is their accuracy when compared with historically observed data; hence various bias correction methods have been developed. However, the coherence between projections and observations, in terms of their statistical properties, has not yet been fully explored. The ability of climate models regarding reproducing the observed changes and trends of main hydrological variables needs to be carefully scrutinised.

In this chapter, a total number of 18 climate projections datasets from the CMIP5 project as well as the observed datasets of precipitation in a large study area (Iraq) to include more pixel of climate variable are selected. Overall, 65 years (1941-2005) of the data collected from 30 rain gauges' stations have been investigated. The Mann Kendall test is used to evaluate the strength and the significance of the trends (if any) in both the simulations and the observations. Furthermore, several exploratory techniques including relative standard deviation p-p plot and Cullen and Frey graph are used to identify the similarity (or disagreement) in data distribution drawn from both datasets.

These findings cast doubts over the reliability of using the projected precipitation directly as the forcing field for conducting impact studies on hydrological processes due to climate change. It is also suggested that more case studies with more datasets need to be carried out to consolidate the findings and gain insight into developing new methodology in this area.

\subsection{Introduction}

Studying precipitation trend is an essential step in assessing climate change impact on hydrological processes. A substantial change of precipitation can lead to a more severe condition of flooding and drought. It is also important to examine such trends 
for water resources planners since they may affect water demand and hence strategies and operations for water supply.

While the trend of recorded observations of hydrological variables such as precipitation has been widely studied using long-term observation dataset (e.g., Zhao et al., 2014); scenario-based climate projections are still a preferred source for most (if not all) studies on future trend linked to climate change. More recently, the Fifth Climate Model Inter-comparison Project CMIP5 (Meinshausen et al., 2011) has published a rich set of climate simulations produced by several large metrological centres in the world, which offers an updated, improved (both accuracy and resolution) collection of climate model outputs to many downstream impact studies, e.g., (Ficklin et al., 2013; Chattopadhyay and Jha, 2016; Jin and Sridhar, 2011; Chattopadhyay, 2014; Abdo et al., 2009).

The post-industrial period, particularly for the $20^{\text {th }}$ century, has been the main focus of many studies on the trend of climatic variables, by and large motivated to establish the link between the so-called anthropogenic greenhouse effect with the change of climate as indicated by the key variables. Moreover, the so-called baseline periods are chosen by many studies as observations records started to become abundant.

The areas studied ranges from global to regional scale. To name just a few: New et al. (2001) show that precipitation has significantly changed in various part of the world during the $20^{\text {th }}$ century. Xu et al. (2005) and Griggs and Noguer (2002) point out that mean annual precipitation during the $20^{\text {th }}$ century considerably increased by (7-12) $\%$ in the high and middle latitude $\left(30^{\circ}-85^{\circ}\right)$ in the northern hemisphere. Philandras et al. (2011) investigate precipitation for a long-term 1901-2009 in the Mediterranean area, where general negative trends were detected. Bocheva et al. (2009) studied extreme rainfall over 40 years (1961-2005) in Bulgaria, and found that extreme events became more frequent during last 15 years of the period, compared with less frequent occurrences of a moderate and weak event.

Over the years, various statistical techniques have been developed to detect the trend and the shift of such in climatological variables, as reported in Martinez et al. (2012) 
and Tabari et al. (2011). There is two leading families of methods: parametric and non-parametric test of trends. Usually, non-parametric methods are preferred over the parametric one (Sonali and Nagesh, 2013) because they are less affected by outliers and do not assume a predefined distribution for the dataset or homogeneity.

The non-parametric Mann-Kendall (MK) statistical test (Mann, 1945; Kendall, 1975) has frequently been used to quantify the significance of trends in precipitation time series (Martinez et al., 2012; Modarres and Silva, 2007; Modarres and Sarhadi, 2009; Tabari et al., 2011). One of the drawbacks of the Mann-Kendall test is that it does not reveal the magnitude of slope (of the trend). That led to the development of another non-parametric test - the Theil-Sen slope technique (Sayemuzzaman and Jha, 2014) which is initially established by Theil (1950) and Sen (1968). This approach gives a more robust estimation of the slope than the least square methods as it is insensitive to extreme values and outliers (Hirsch et al., 1982; Breidt, 2005). Additionally, there are also fewer implicit assumptions about the data structure considering them robust against departures from normality (Hirsch and Helsel, 1992).

Compared with those studies, there is hardly any in the literature focusing on the trend analysis of those variables in future; most studies, however, used "snapshots" from climate projections to indicate the difference (hence change) between the projected variable and its current property, without revealing the process (the temporal trend) associated with such change. On the other hand, researchers tend to use the projected, scenario-defined variable, notably precipitation to drive other (hydrological) models for the impact study. The errors or biases in these simulated variables have been widely recognised in this kind of applications. Sophisticated bias correction methods have also been developed to cope with this situation. But again, rarely any attention is put on the trend of those simulated variables either with or without bias correction.

In this chapter, it is argued that:

1) The ability of climate model in reproducing the observed trend in the baseline is at least as necessary as that for accuracy in the simulation. As such, such ability needs to be verified (improved where possible) before any other application make use of it to study future impact; 
2) The trend analysis of the projected variable from a well-performed climate model in capturing observed trend should be able to offer more insights into the changes of the variable in future; and

3) This combined trend analysis can be used as an objective index to measure whether a climate model projection is more statistically reliable along with the conventional accuracy measurement.

\subsection{Study area}

The climate projections from the CMIP5 project have a spatial resolution of 1 degree 1 degree $(\approx 100 \mathrm{~km})$ and coarser. For this reason, a larger study area, Iraq, is selected to include many rain gauges and perform the comparison. Iraq is located in the southwest of the Asian continent and shares boundaries with each Syria and Jordan from the west, Turkey from the north, Iran from the east, and Saudi Arabia and Kuwait from the south (Fig. 8-1). Iraq comprises a total area of 437,065 km².

The climate in Iraq is mainly of the continental, subtropical semi-arid type, with the north and north-eastern mountainous regions having a Mediterranean climate (FAO, 2003). Rainfall is very seasonal and occurs in the winter from December to February, except in the north and northeast of the country, where the rainy season is from November to April. Iraq's elevation reduces from the north and the northeast where the mountains are near the Turkish and Iranian borders $(3,450 \mathrm{~m})$ to the west and south where the desert near Saudi Arabia and Syrian borders (few meters).

The average annual rainfall is estimated to be $216 \mathrm{~mm}$ but ranges from 1,200 $\mathrm{mm}$ in the northeast to less than $100 \mathrm{~mm}$ over 60 per cent of the country in the south (FAO, 2003). Winters are cool to cold, with a day temperature of about $16{ }^{\circ} \mathrm{C}$ dropping at night to $2{ }^{\circ} \mathrm{C}$ with a possibility of frost. Summers are dry and hot to extremely hot, with a shade temperature of over $43{ }^{\circ} \mathrm{C}$ during July and August, yet dropping at night to $26^{\circ} \mathrm{C}$ (Ajaaj et al., 2016). 


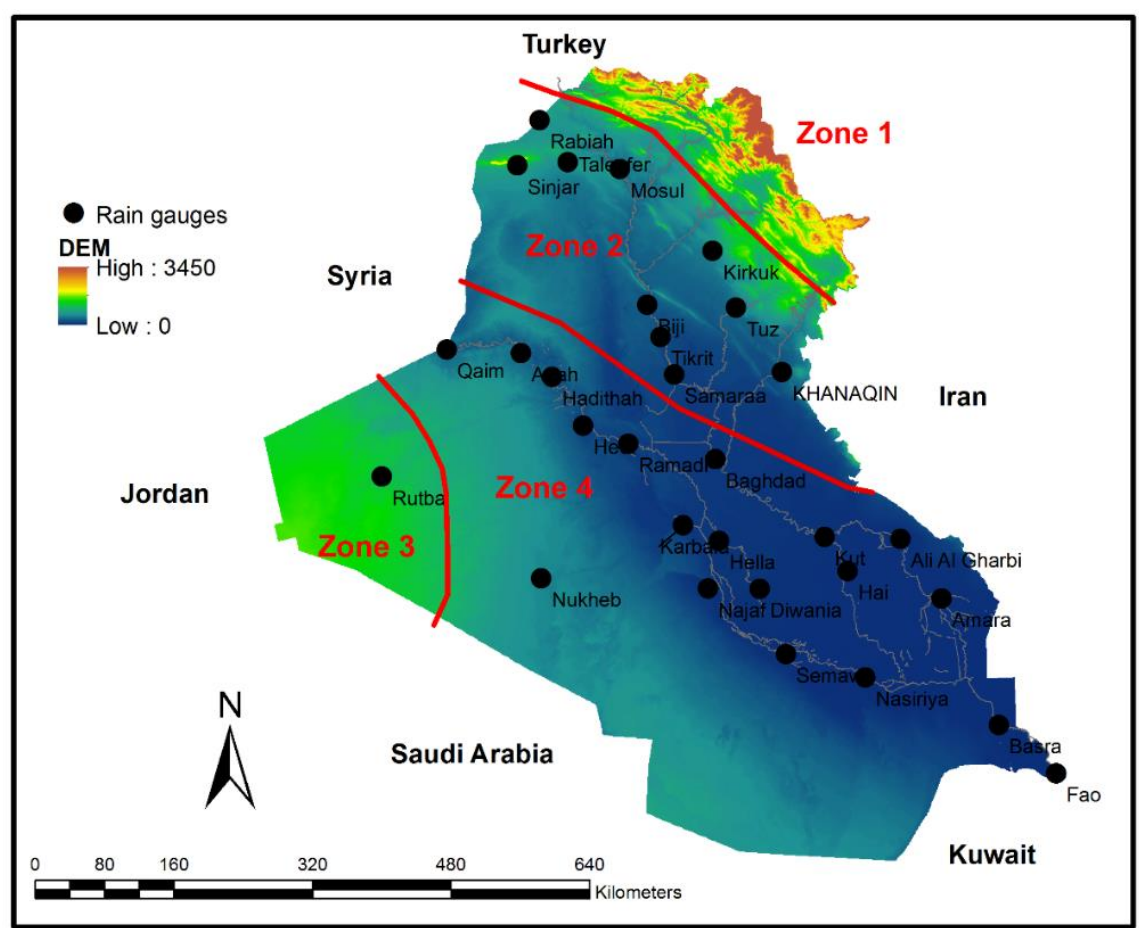

Fig. 8-1 Elevation map of Iraq with climatic zones

Iraq can be divided into four agro-ecological zones (FAO, 2003):

1) Arid and semi-arid zones with a Mediterranean climate (Zone 1 in Fig. 8-1): Annual precipitation varies between 700-1000 $\mathrm{mm}$ and occurs between October and April. The weather has cold and rainy winters, while summers are hot and dry even torrid up to quite high altitudes. This zone covers mainly the northern region, mountains of Iraqi Kurdistan (Zagros and Taurus). This is the only region in Iraq that receives a considerable amount of precipitation;

2) Steppes with winter rainfall of 200-400 mm annually (Zone 2 in Fig. 8-1): Summers are extremely hot and winters cold. The climate is arid, but in the cold half of the year, for a few months, some depressions can pass, bringing moderate rainfall. This zone is located between the Mediterranean zone and the desert zone;

3) The desert zone/ North-west of Mesopotamia (Zone 3 in Fig. 8-1): with extreme summer temperatures and less than $200 \mathrm{~mm}$ of rainfall annually. the climate is desert, quite cold in winter, with frequent frosts, and hot in summer; and 
4) The irrigated area which extends between the Tigris and Euphrates rivers (Zone 4 in Fig. 8-1): The climate is desert or semi-desert, with mild winters, which becomes progressively warmer as you head south; summers are extremely hot. This area extends from the north of Baghdad to Basra in the south.

Table 8-1: Iraqi rain gauges' stations used in this study

\begin{tabular}{cccccccccc}
\hline Station & $\begin{array}{c}\text { Station } \\
\text { ID }\end{array}$ & Lat. & Lon. & $\begin{array}{c}\text { Altitude } \\
(\mathbf{m})\end{array}$ & Station & $\begin{array}{c}\text { Station } \\
\text { ID }\end{array}$ & Lat. & $\begin{array}{c}\text { Long. } \\
\text { Altitude } \\
(\mathbf{m})\end{array}$ \\
\hline Sinjar & R1 & $36.32^{\circ}$ & $41.83^{\circ}$ & 583 & Diwaniya & R16 & $31.95^{\circ}$ & $44.95^{\circ}$ & 20 \\
Telaefer & R2 & $36.37^{\circ}$ & $42.48^{\circ}$ & 373 & Ramadi & R17 & $33.45^{\circ}$ & $43.32^{\circ}$ & 48 \\
Najaf & R3 & $31.95^{\circ}$ & $44.32^{\circ}$ & 53 & Tuz & R18 & $34.88^{\circ}$ & $44.65^{\circ}$ & 220 \\
Qaim & R4 & $34.38^{\circ}$ & $41.02^{\circ}$ & 178 & Samaraa & R19 & $34.18^{\circ}$ & $43.88^{\circ}$ & 75 \\
Anah & R5 & $34.37^{\circ}$ & $41.95^{\circ}$ & 175 & Amara & R20 & $31.83^{\circ}$ & $47.17^{\circ}$ & 9 \\
Nukheb & R6 & $32.03^{\circ}$ & $42.28^{\circ}$ & 305 & Mosul & R21 & $36.31^{\circ}$ & $43.15^{\circ}$ & 223 \\
Hai & R7 & $32.13^{\circ}$ & $46.03^{\circ}$ & 17 & Rutba & R22 & $33.03^{\circ}$ & $40.28^{\circ}$ & 222 \\
Semawa & R8 & $31.27^{\circ}$ & $45.27^{\circ}$ & 11 & Tikrit & R23 & $34.57^{\circ}$ & $43.70^{\circ}$ & 107 \\
Heet & R9 & $33.63^{\circ}$ & $42.75^{\circ}$ & 58 & Biji & R24 & $34.90^{\circ}$ & $43.53^{\circ}$ & 116 \\
Rabiah & R10 & $36.80^{\circ}$ & $42.10^{\circ}$ & 382 & Haditha & R25 & $34.13^{\circ}$ & $42.35^{\circ}$ & 108 \\
Hella & R11 & $32.45^{\circ}$ & $44.45^{\circ}$ & 27 & Fao & R26 & $29.98^{\circ}$ & $48.50^{\circ}$ & 1 \\
Baghdad & R12 & $33.30^{\circ}$ & $44.40^{\circ}$ & 32 & Khanaqin & R27 & $34.21^{\circ}$ & $45.23^{\circ}$ & 202 \\
Nasiriya & R13 & $31.02^{\circ}$ & $46.23^{\circ}$ & 5 & Basra & R28 & $30.50^{\circ}$ & $47.83^{\circ}$ & 2 \\
& & & & & Ali & & & & \\
Kut & R14 & $32.49^{\circ}$ & $45.75^{\circ}$ & 21 & AlGharbi & R29 & $32.46^{\circ}$ & $46.68^{\circ}$ & 13 \\
Kirkuk & R15 & $35.47^{\circ}$ & $44.35^{\circ}$ & 331 & Karbalaa & R30 & $32.61^{\circ}$ & $44.01^{\circ}$ & 29
\end{tabular}

\subsection{Data}

Monthly Precipitation data from 30 rain gauges over the period of 1941-2005 are collected and obtained from the General Organisation of Meteorology and Seismic Monitoring in Iraq are illustrated in Fig. 8-1 and Table 8-1. The missing datasets are filled using inverse distance weighted interpolation method (IDW). The Statistical summary of annual precipitation is illustrated in Fig. 8-2 for zones 2, 3 and 4. Yearly rainfall for zone 2 ranges from 35 to $700 \mathrm{~mm}$ with the average of $(\sim 300 \mathrm{~mm})$ for the 
period of 1941-2005. Whereas, for zone 3 and 4 the range is $3-347 \mathrm{~mm} /$ year and average of ( 127 mm/year).

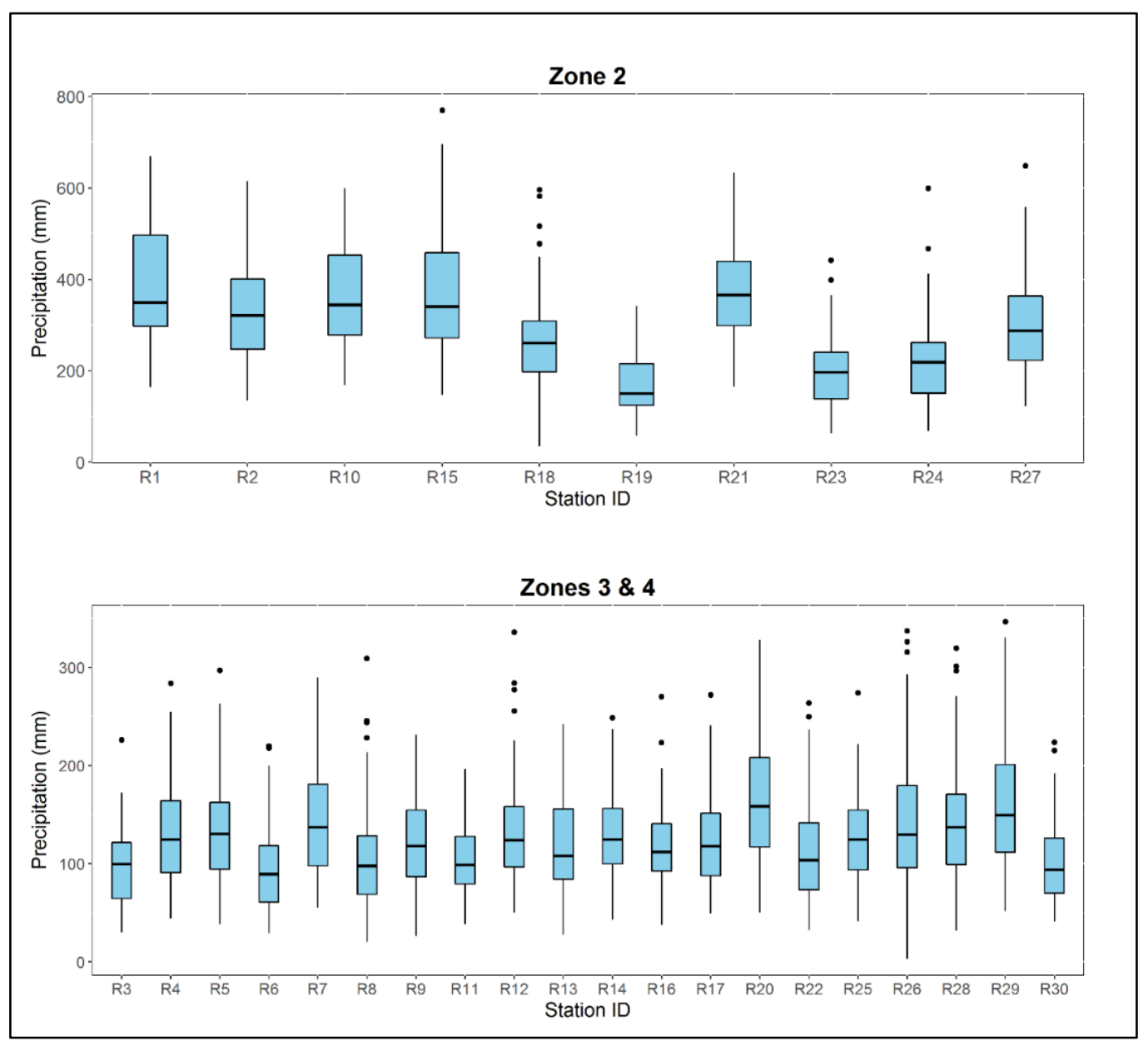

Fig. 8-2 Box plot of annual observed precipitation in Iraq

The average precipitation is obtained using the Thiessen polygon method based on 30 rain gauges as illustrated in Fig.8-3. Additionally, the boxplot of average rainfall in Iraq for seven decadal periods is examined as follow: 1941-1950, 1951-1960, 19611970, 1971-1980, 1981-1990, 1991-2000 and 2001-2005. The boxplot in Fig. 8-4 is used to investigate the annual and seasonal patterns [winter (combination of December, January and February DJF)], Spring [sum of March, April and May (MAM)] and Autumn [sum of September, October and November (SON)] of average precipitation obtained from 30 stations based on 7 temporal bands defined previously. 


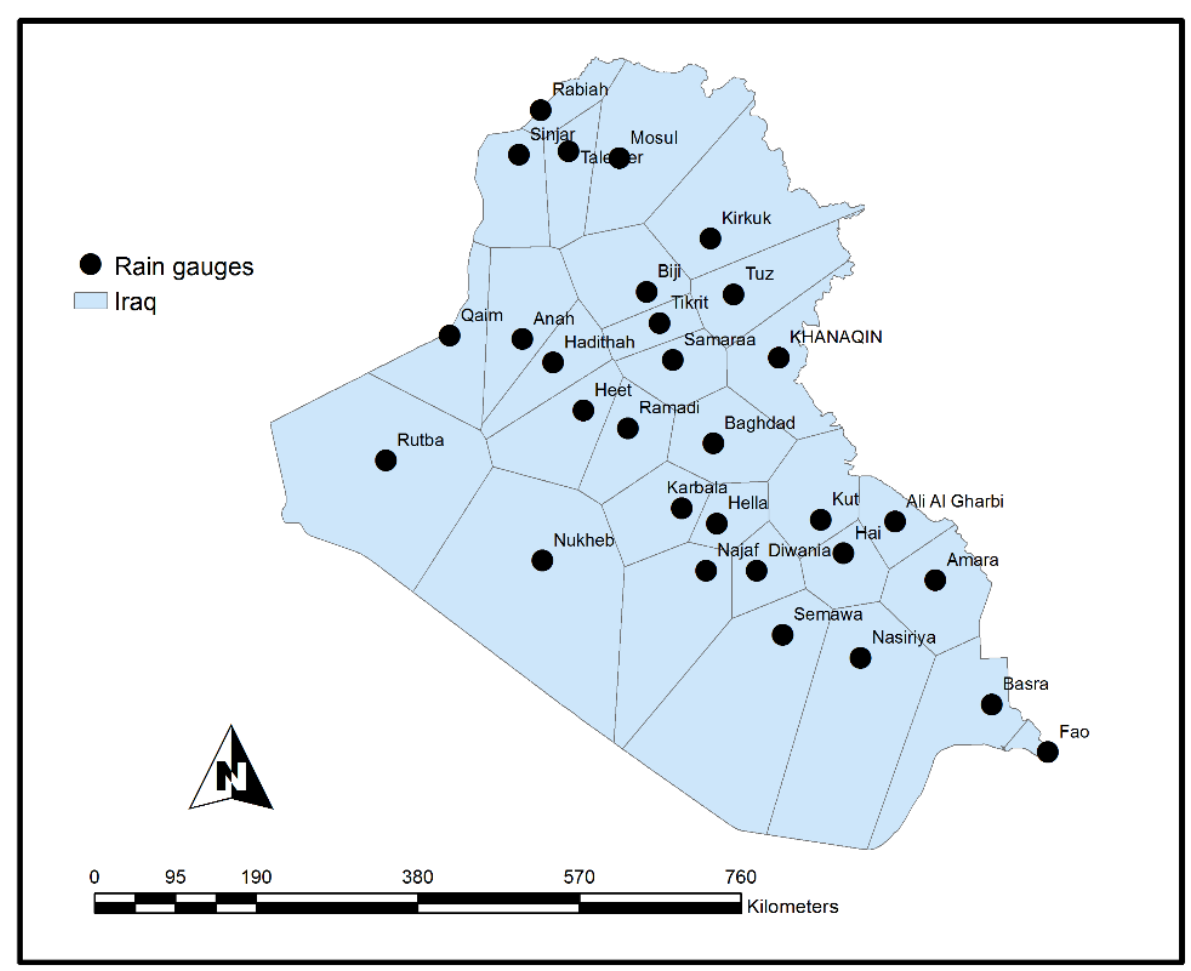

Fig. 8-3 Thiessen polygon for observed rainfall in Iraq

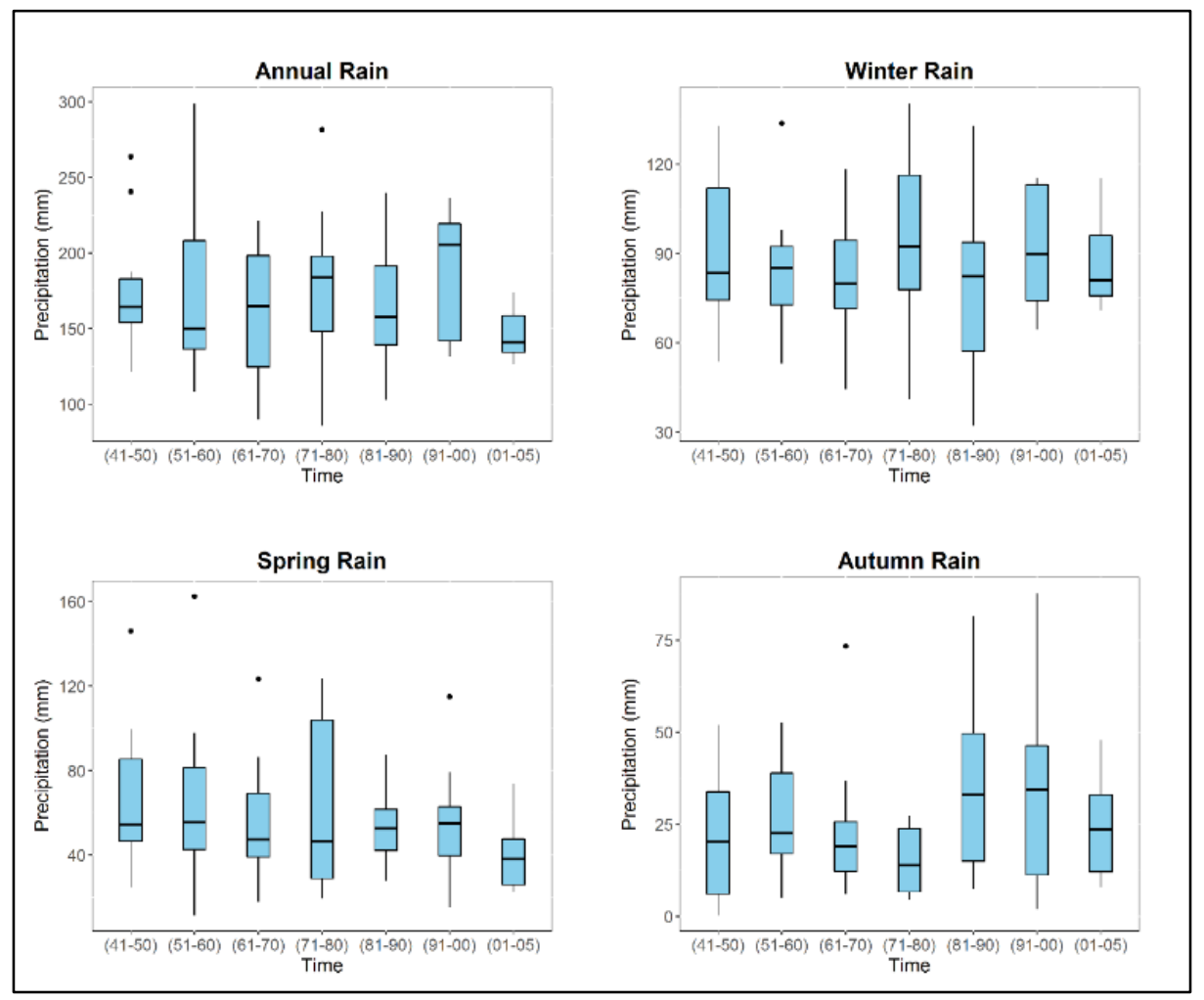

Fig.8-4 Box plots of average precipitation in Iraq for different temporal bands for period 1941-2005 
It can be seen in Fig. 8-4 that the pattern of rainfall changes at different temporal bands and seasons.

Table 8-2: The CMIP5 monthly models used in this study

\begin{tabular}{llc}
\hline Model & Institution & $\begin{array}{c}\text { Spatial } \\
\text { Resolution (Lat. } \\
\times\end{array}$ \\
\hline Long.)
\end{tabular}

The Coupled Model Inter-Comparison Phase five CMIP5 experiments consist of a number of numerical climate model's simulations with various constraints such as land-use changes, environmental pollution and volcanic emissions. CMIP5 (Meinshausen et al., 2011) are divided into two major components:

1) Long-term experiments (century and longer); and

2) Near-term experiments (decadal prediction). 
Twenty-eight centres in the world are providing CMIP5 climate model outputs with different sets of model outputs near and extended future scenarios and different groups of spatial and temporal resolution. In this study, a total number of 18 models from CMIP5 based on long-term scenarios are used. There are four main scenarios of future climate data: RCPs 2.6, RCPs 4.5, RCPs 6.0 and RCPs 8.5 (Meinshausen et al., 2011). Detailed information of the CMIP5 models used in this study is described in Table 8-2.

\subsection{Methods}

\subsubsection{The goodness of fit tests (GOF)}

In this chapter, five statistical GOF tests are used to evaluate the selected model simulations of CMIP5 against observed precipitation based on monthly time series for the period of 1941-2005. The criteria used for the evaluation are:

1) Mean Error (ME) which can be determined as follow:

$$
M E=\frac{1}{n} \sum_{i=1}^{n}\left(p_{s, t}-p_{o, t}\right)
$$

where $p_{o, t}$ is the observed rainfall at time $t$ and $p_{s, t}$ is the simulated rainfall at time $t$.

2) Mean Absolute Error (MAE) that can be computed as:

$$
M A E=\frac{1}{n} \sum_{i=1}^{n}\left|p_{s, t}-p_{o, t}\right|
$$

3) Root Mean Square Error (RMSE) that can be calculated as: 


$$
R M S E=\sqrt{\frac{1}{n} \sum_{i=1}^{n}\left(p_{s, t}-p_{o, t}\right)^{2}}
$$

4) Correlation Coefficient ( $r$ ): This can measure the linear relationship between two time series variables. This value ranges from -1 which indicates a perfect negative correction to 0 which is there is no correlation at all, and to 1 (perfect positive relationship). It can be computed as follow:

$$
r=\frac{\sum_{t=1}^{T}\left(p_{o, t}-\overline{p_{o}}\right)\left(p_{s, t}-\overline{p_{s}}\right)}{\sum_{t=1}^{T}\left[\left(p_{o, t}-\overline{p_{o}}\right)^{2}\right]^{0.5} \sum_{t=1}^{T}\left[\left(p_{s, t}-\overline{p_{s}}\right)^{2}\right]^{0.5}}
$$

5) Fit Probability Distributions: It is imperative to know the underlining distributions of both observations and simulated data. It serves two purposes:

a) To see if the two data sets are statistically consistent; and

b) To identify any changes in the probability distribution of simulated data for future.

The Skewness-kurtosis graph technique is applied to select the most suitable distribution type to fit the data set. The detail of this method can be referred to Cullen and Frey (1999). This method makes use of skewness versus kurtosis graph to check the similarity between the samples (observed and simulated data) and those from a predefined set of distributions, by showing the location calculated from the samples on the graph whose background is failed with those curves generated from the predefined distributions.

Other commonly used techniques such as probability-probability (P-P) plot, quantilequantile (Q-Q) plot, density plot and Cumulative Distribution Functions CDFs are also used to measure the goodness of fit of the select distribution. In this study, eight predefined probability distributions are considered including beta, uniform, exponential, gamma, logistic, log-normal, normal and Weibull distributions. An R package 'fitdistrplus' (Delignette-Muller and Dutang, 2014) is employed to analyse both the observed and selected CMIP5 models. 


\subsubsection{Bias correction: quantile mapping}

The bias correction of the quantile is applied for the best-selected models from CMIP5 models based goodness of fit criteria mentioned in section 8.4.1. Many studies such as Maraun (2013) and Eden et al. (2012) have found that climate projection simulation from Global Climate Models (GCMs) often come with substantial amount uncertainties as well as biases and errors. Undoubtedly, the confidence in the direct use of GCMs simulations has been adversely affected such that no reliable conclusions can be drawn using uncorrected GCMs simulation data. However, sophisticated bias and error correction of GCMs data have gone beyond the scope of this study. The simple Quantile Mapping (Maraun et al., 2010) technique is used to adjust the climate data over the baseline period and then apply the same Quantile Mapping model to study the trend using both corrected and uncorrected GCMs.

Quantile mapping is a bias correction technique which the modelled variable is changed through equating the cumulative distribution functions CDFs of both observation and simulated dataset. For that matter, the following transform function is implemented:

$$
\hat{X}_{m, p}=F_{o, h}^{-1}\left\{F_{m, h}\left[X_{m, p}(t)\right]\right\}
$$

where $F_{o, h}$ and $F_{m, h}$ are cumulative distribution functions of both the observed and the simulated time series, $X_{m, p}(t)$ is the modelled variable at time $(\mathrm{t})$. Typically, the quantile mapping algorithm is presented through quantile-quantile (Q-Q) plot (e.g. scatter plot between empirical quantile of simulated and observed data if the CDF (simulated data) and inverse CDF (observed data) will be empirically projected from the data.

Similar to all statistical bias correction approaches, the quantile mapping method presumes that the climate model's biases are stationary for both the historical and the future scenarios. In other words, the correction of data for the historical period can be 
utilised for the future period. Further information about this method of bias correction can be found in Maraun et al. (2010). Practically, quantile mapping is implemented through fitting parametric transformations to the quantile-quantile relation of observation and modelled dataset, and then the transformation is employed to adjust the distribution of the climate model data to match the distribution of the observed data.

\subsubsection{Mann Kendall trend test (MK)}

The non-parametric trend test, Mann-Kendall (Mann, 1945; Kendall, 1975) is extensively used in hydrology and climatology to investigate significance slope or trend since it is simple and its robustness. Let considering $\mathrm{X}=\left(\mathrm{x}_{1}, \mathrm{x}_{2}, \mathrm{x}_{3} \ldots, \mathrm{x}_{\mathrm{n}}\right)$ is a time series dataset, the Mann-Kendall statistics $S$ can be computed as follow:

$$
S=\sum_{i=1}^{n-1} \sum_{j-i+1}^{n} \operatorname{sgn}\left(x_{j}-x_{i}\right)
$$

where:

$$
\operatorname{sgn}\left(x_{j}-x_{i}\right)=\left\{\begin{array}{c}
+1 \text { if }\left(x_{j}-x_{i}\right)>0 \\
0 \text { if }\left(x_{j}-x_{i}\right)=0 \\
-1 \text { if }\left(x_{j}-x_{i}\right)<0
\end{array}\right.
$$

The variance for the Mann- Kendall trend test can be calculated as follow:

$$
\operatorname{Var}_{s}=\frac{1}{18}\left[n_{i}\left(n_{i}-1\right)\left(2 n_{i}+5\right)-\sum_{p=1}^{g i} t_{i p}\left(t_{i p}-1\right)\left(2 t_{i p}+5\right)\right]
$$

where:

$n_{i}:$ is the number of data points

$g_{i}$ : is the number of tied groups for the $i$ th month

$t_{i} p$ : is the number of data in the $p_{t h}$ group for the $i_{t h}$ month. 
Correspondingly, the $Z$ statistic is defined in the equation:

$$
Z=\left\{\begin{array}{l}
\frac{S-1}{\sqrt{\operatorname{Var}_{S}}} \text { if } S>0 \\
0 \text { if } S=0 \\
\frac{S+1}{\sqrt{\operatorname{Var}_{s}}} \text { if } S<0
\end{array}\right.
$$

It can be revealed that following the Null Hypothesis (no trend) $\mathrm{H}_{0}$ that $\mathrm{S}$ will be following in normal distribution and thus, this can be used to test the hypothesis with a certain confidence level of $\alpha / 2$. The overall projected trend slope $\beta$ which is TheilSen slope (Sen, 1968; Theil, 1950) for measured dataset $Y$ over time $X$. The individual slope estimator is calculated as follow:

$$
Q i=\frac{Y_{j}-Y_{i}}{X_{j}-X_{i}}
$$

The Mann-Kendall trend test will be carried out using ' $r k t$ ' package (Marchetto, 2015) in R statistical programme.

\subsection{Results and discussion}

\subsubsection{Statistical comparison of the observed and the modelled precipitations}

The technique of Skewness-kurtosis graph (Cullen and Frey plot) is employed to check whether the areal average of observed and modelled precipitation over Iraq are from the same family of the probability distribution. The monthly time series of the observations and the 18 models of CMIP5 are evaluated against eight theoretical distributions. 


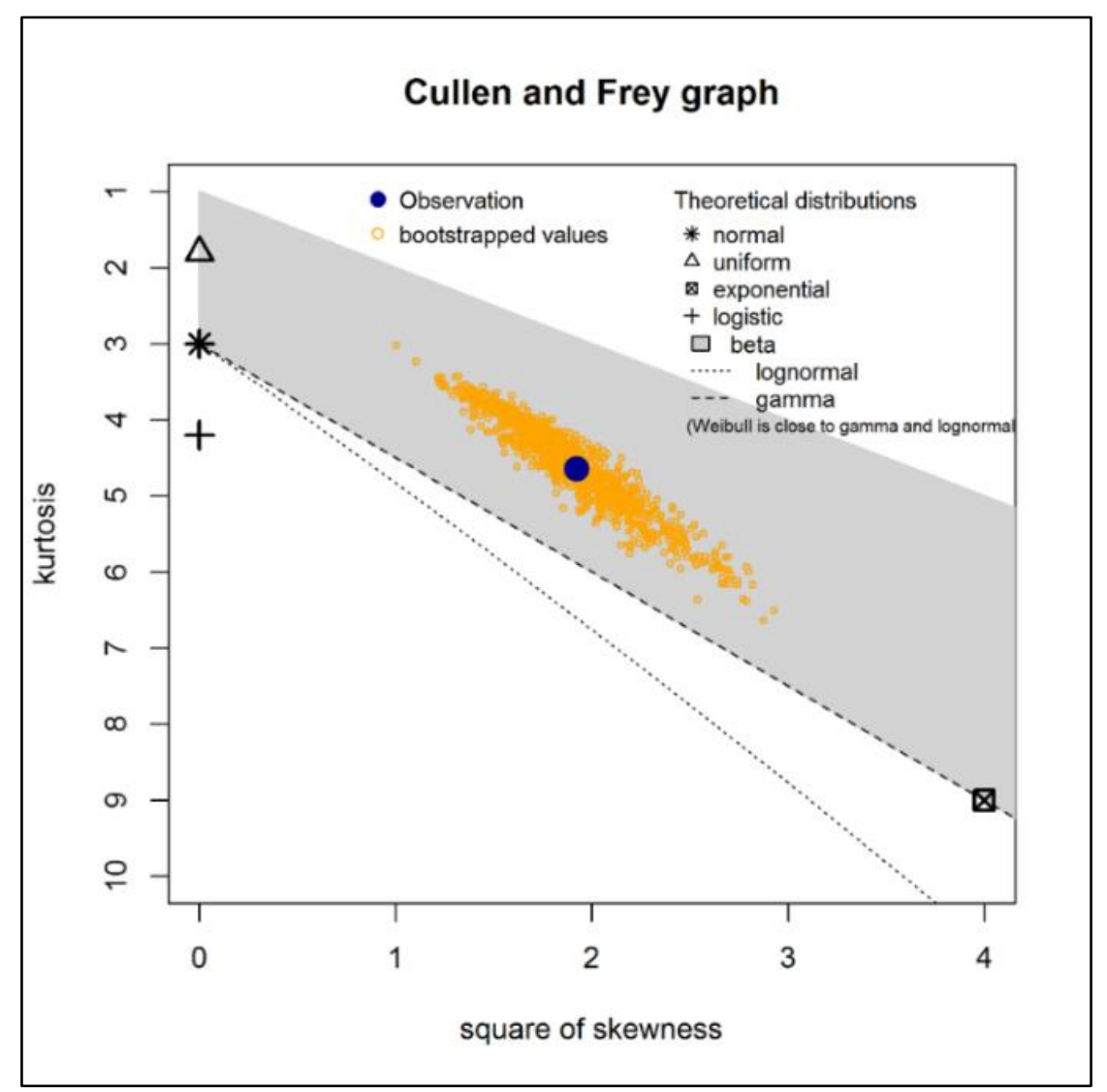

Fig. 8-5 Cullen and Frey graph of areal average monthly precipitation for the observations

As it can be seen in Fig. 8-5, the observed rainfall lies within the region of beta distribution. Further assessment of the suitability of the observed precipitation to beta is conducted using Q-Q plot, p-p plot, empirical, theoretical densities and cumulative density function CDFs as in Fig. 8-6. The results show that observation fits well into beta distribution. The 18 models of CMIP5 are evaluated based on ME, MAE, RMSE, $r$ and fit theoretical distribution for monthly areal average rainfall of Iraq as illustrated in table 8-3. The comparison reveals that bcc-csm1-1, bcc-csm1-1-m, CCSM4, MIROC5 and MRI-CGCM3 models have a relatively better representation of rainfall than other models.

The cumulative rainfall for the areal average was calculated in Iraq from the annual rainfall for the observations and simulated results of CMIP5 as shown in Fig. 8-7. The 
comparison reveals that the models of MRI-CGCM3, CCM4 and MIROC5 have a better qualitative estimation than others models as illustrated in Fig. 8-7 and the statistical comparison in Table (8-3) also supports this claim.

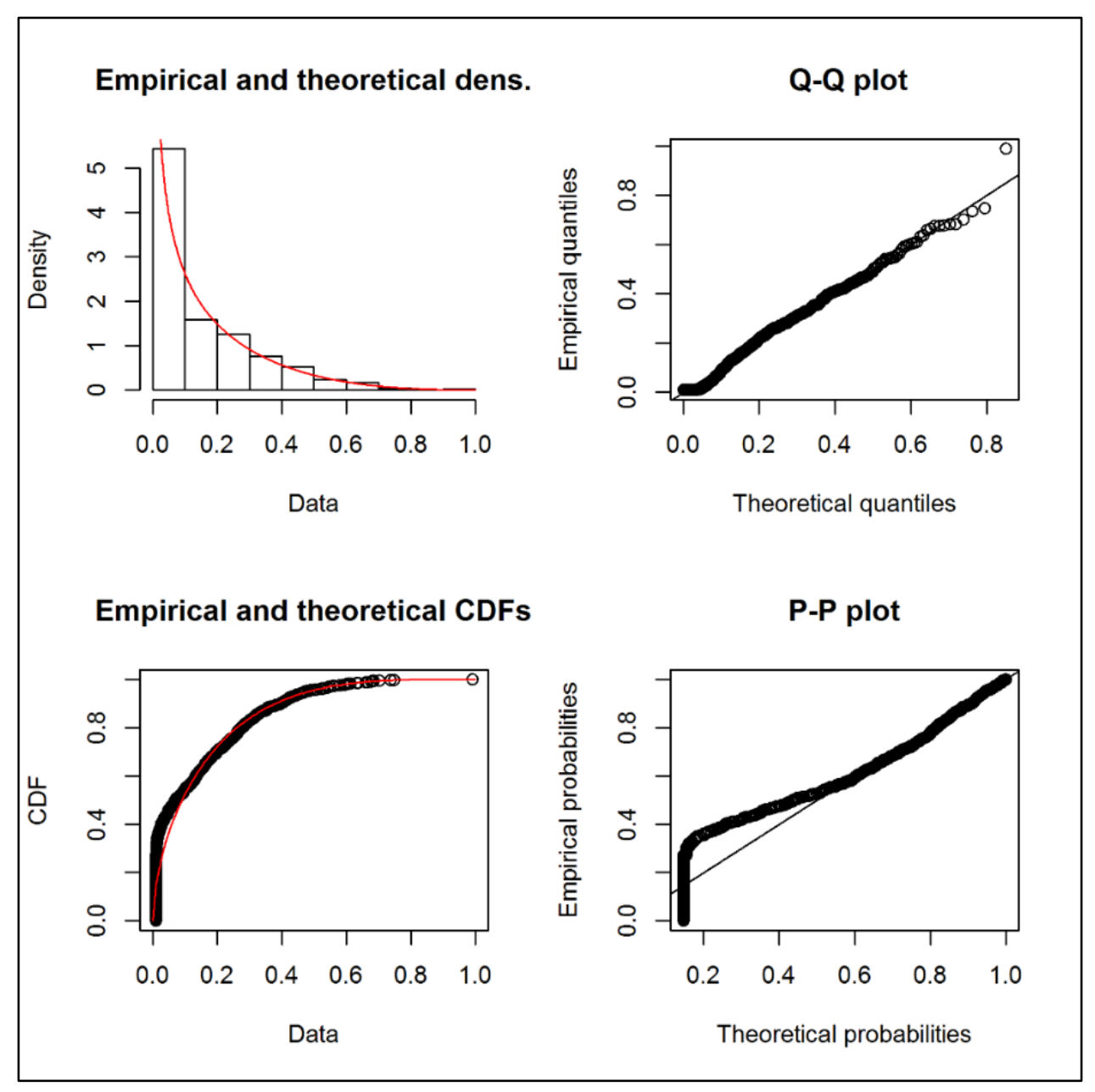

Fig. 8-6 Goodness of fit of the observed monthly areal average precipitation over Iraq for the beta distribution

The technique of quantile mapping QM is conducted on the monthly rainfall time series of the five selected models bcc-csm-1-1, bcc-csm-1-1-m, CCM4, MIROC5 and MRI-CGCM3. For every location of rain gauges (30 stations), the monthly precipitation is corrected where QM is carried out where January data of simulated rainfall is corrected against observation of January, February against February and so on. 
Table 8-3: GOFs of CMIP5 monthly areal average rainfall models against ob

\begin{tabular}{|c|c|c|c|c|c|c|c|c|c|c|c|c|}
\hline Model & 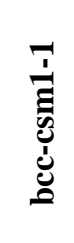 & 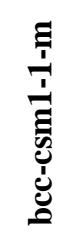 & 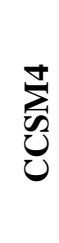 & 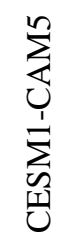 & $\begin{array}{l}b_{1}^{1} \\
\sum_{0}^{1} \\
\sum_{1}^{1} \\
0 \\
0=1 \\
0\end{array}$ & 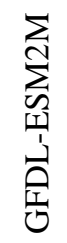 & $\begin{array}{l}1 \\
1 \\
\text { In } \\
\vdots \\
\tilde{n} \\
0 \\
0\end{array}$ & 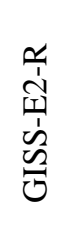 & 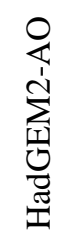 & 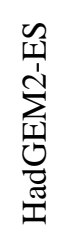 & 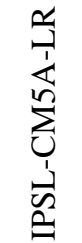 & 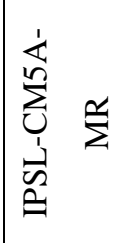 \\
\hline
\end{tabular}

\section{Fit Theoretical Distribution}

\begin{tabular}{|c|c|c|c|c|c|c|c|c|c|c|c|c|}
\hline Beta & Beta & Beta & ---- & ---- & ---- & ---- & Beta & --- & ---- & ---- & ---- & Bet \\
\hline \multicolumn{13}{|l|}{ MAE } \\
\hline 12.80 & 9.98 & 12.19 & 12.02 & 10.34 & 12.97 & 13.49 & 14.82 & 14.46 & 12.41 & 10.82 & 10.76 & 11 \\
\hline \multicolumn{13}{|l|}{$\mathbf{R}$} \\
\hline 0.38 & 0.48 & 0.34 & 0.25 & 0.41 & 0.17 & 0.38 & 0.28 & 0.41 & 0.40 & 0.39 & 0.41 & 0 \\
\hline
\end{tabular}

ME

\begin{tabular}{|l|l|l|l|l|l|l|l|l|l|l|l|l}
\hline-2.70 & 4.86 & 0.39 & 5.28 & 6.01 & 6.77 & -3.75 & -4.95 & -6.97 & -2.71 & 4.75 & 6.96 & 0.3 \\
\hline
\end{tabular}

RMSE

\begin{tabular}{|l|l|l|l|l|l|l|l|l|l|l|l|l}
\hline 19.36 & 16.54 & 18.69 & 18.89 & 17.41 & 20.65 & 19.75 & 22.20 & 25.11 & 20.74 & 18.27 & 17.96 & 16.9 \\
\hline
\end{tabular}



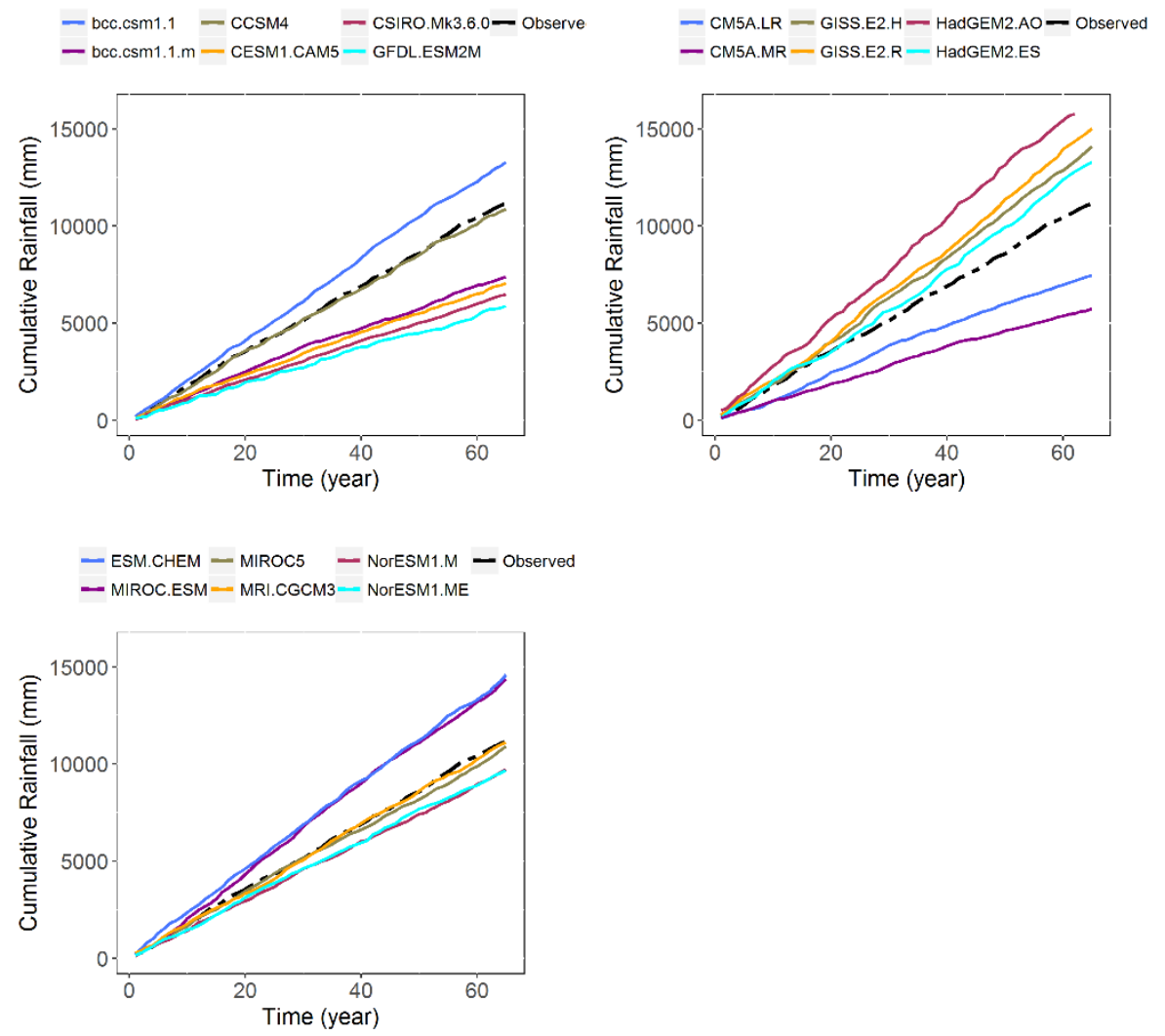

Fig. 8-7 Cumulative of the areal average of observed and modelled precipitation over Iraq

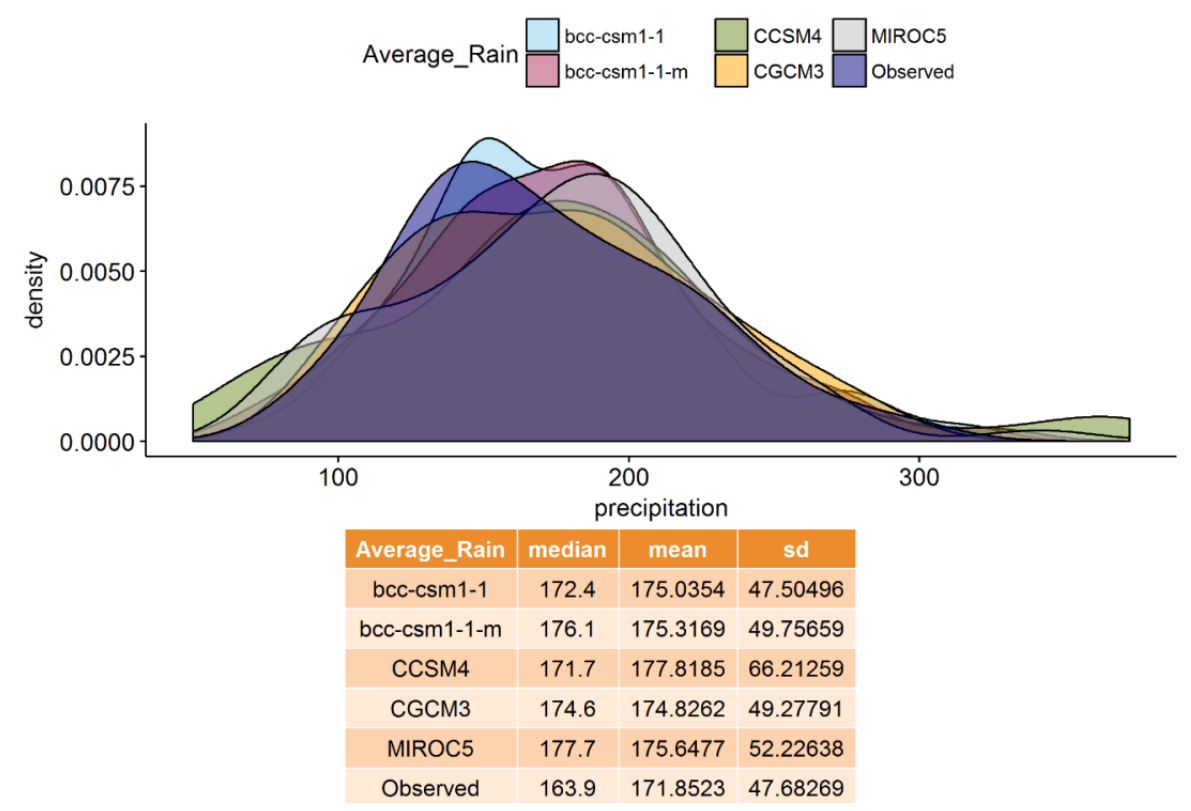

Fig. 8-8 Density plots of the areal average of observed and modelled precipitation over Iraq after bias correction 
The density plots of the areal average of the corrected modelled precipitation and the observation reveal that overlapped on each other as illustrated in Fig. 8-8. Moreover, the statistical parameters of the mean, median and standard deviation $s d$ (as in Fig. 88) shows that modelled and observed rainfall are close to each other.

\subsubsection{Trend analysis of average rainfall}

The trends of annual rainfall for the average condition are evaluated using the Mann Kendall test for the observed and the five selected models bcc-csm-1-1, bcc-csm-1-1m, CCM4, MIROC5 and MRI-CGCM3. The comparison of trends is conducted based on both point by point (as demonstrated in Figures 8-9, 8-10 and 8-11) and the areal average of the study area (Fig. 8-12). The point by point trend comparison at the 27 locations shows that some models such as (bcc-csm-1-1, bcc-csm-1-1-m and CCM4) demonstrate the same trend direction (decrease trend) at 19 sites as revealed in Fig. 89. Meanwhile, the MIROC5 model reveals the same trend direction (mixed of positive and negative trends), the MRI-CGCM3 model shows the same trend direction at 8 locations as shown in Fig. 8-9.

The comparison of the trend also is carried out based on the zones of the study area. Figure 8-10 shows the comparison of trend using Mann Kendall test for zone 2 for the observed and five selected models of CMIP5; it can be concluded most of the modelled rainfall demonstrate the same direction of the trend. Meanwhile, for zones 3 and 4, four of the selected models of CMIP5 reveal the same trend direction as illustrated in Fig. 8-11. 


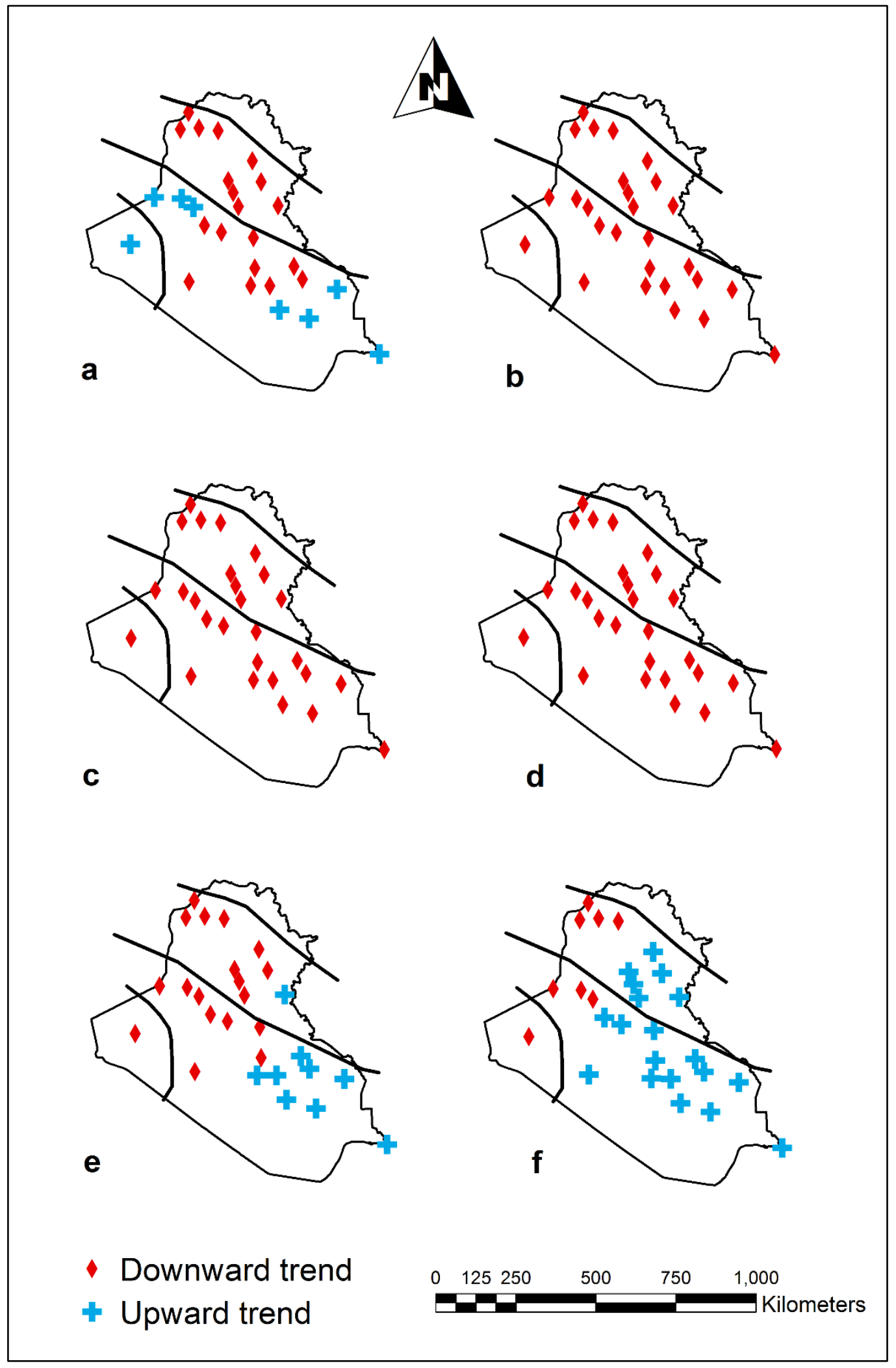

Fig. 8-9 Spatial variation of the annual average rainfall trend using Mann Kendall test over Iraq for a) Observed, b) bcc-csm-1-1 model, c) bcc-csm-1-1-m model, d) CCM4 model, e) MIROC5 model and f) MRI-CGCM3 model 


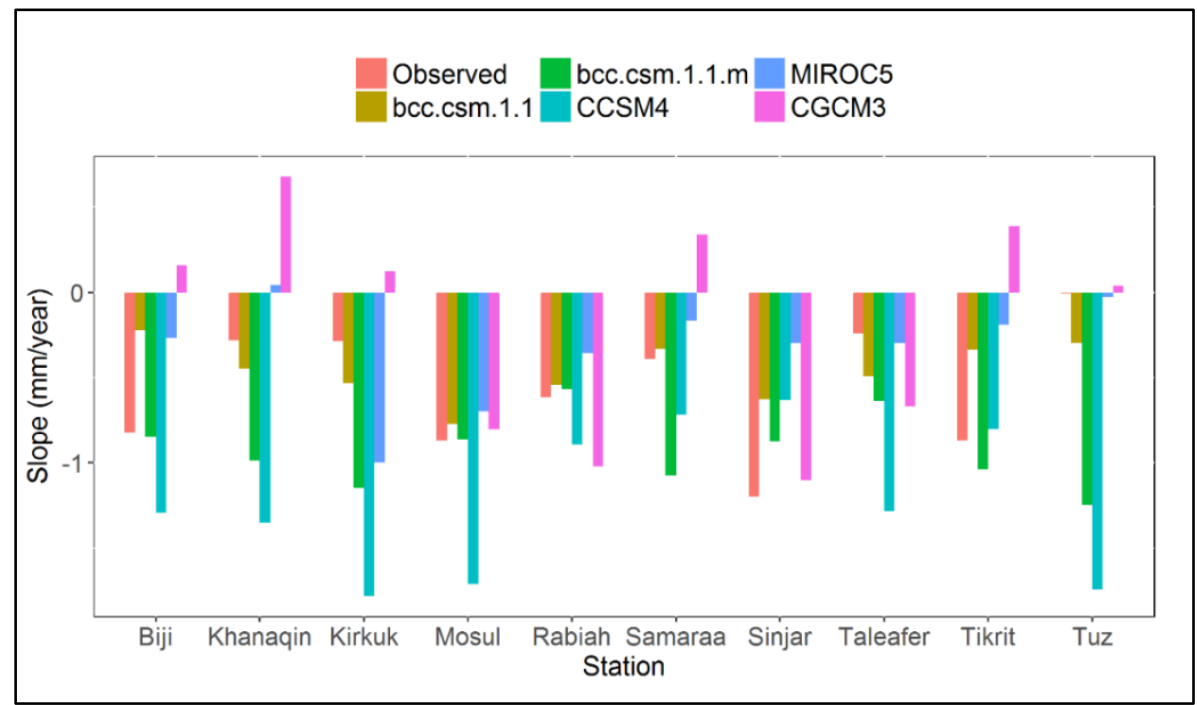

Fig. 8-10 Linear trends of annual rainfall for the observed and simulated time series using the Mann Kendall trend test in zone 2

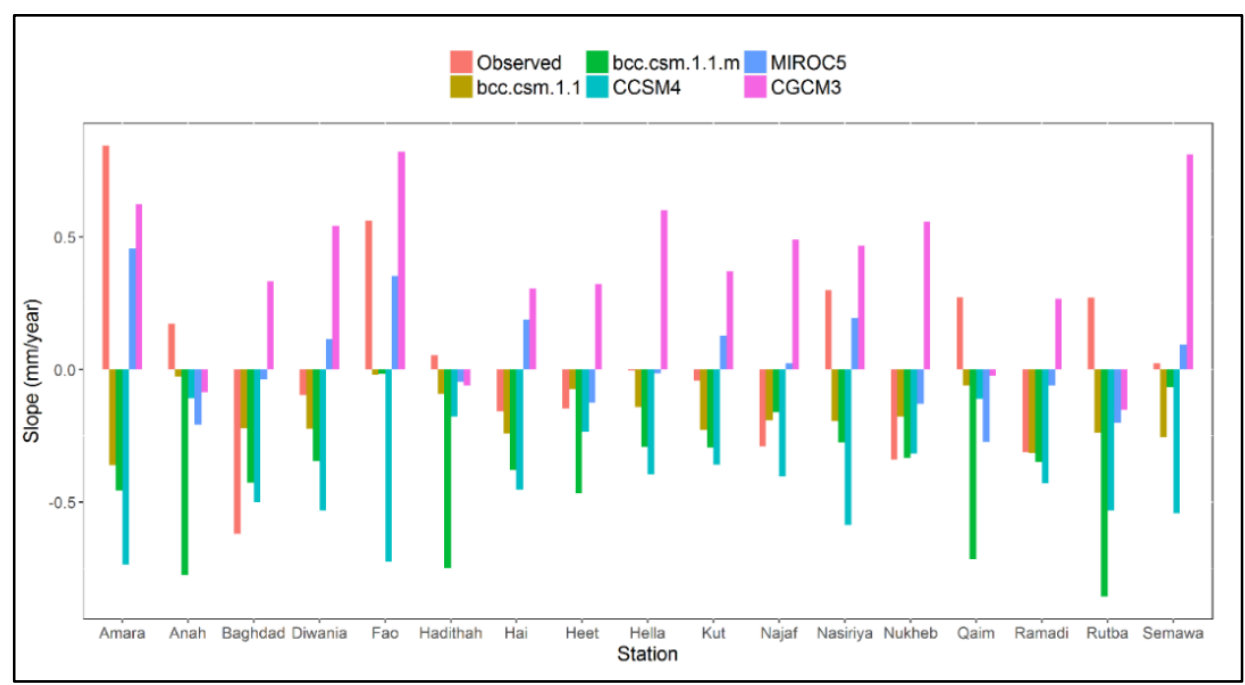

Fig. 8-11 Linear trends of annual rainfall for the observed and simulated time series using the Mann Kendall trend test in zones 3 and 4

Also, the areal average trend of the rainfall for the median condition shows that models of [bcc-csm-1-1, bcc-csm-1-1-m, CCM4 and MIROC5] demonstrate decrease trends, same as observed one. However, the MRI-CGCM3 model shows the opposite trend (positive). 


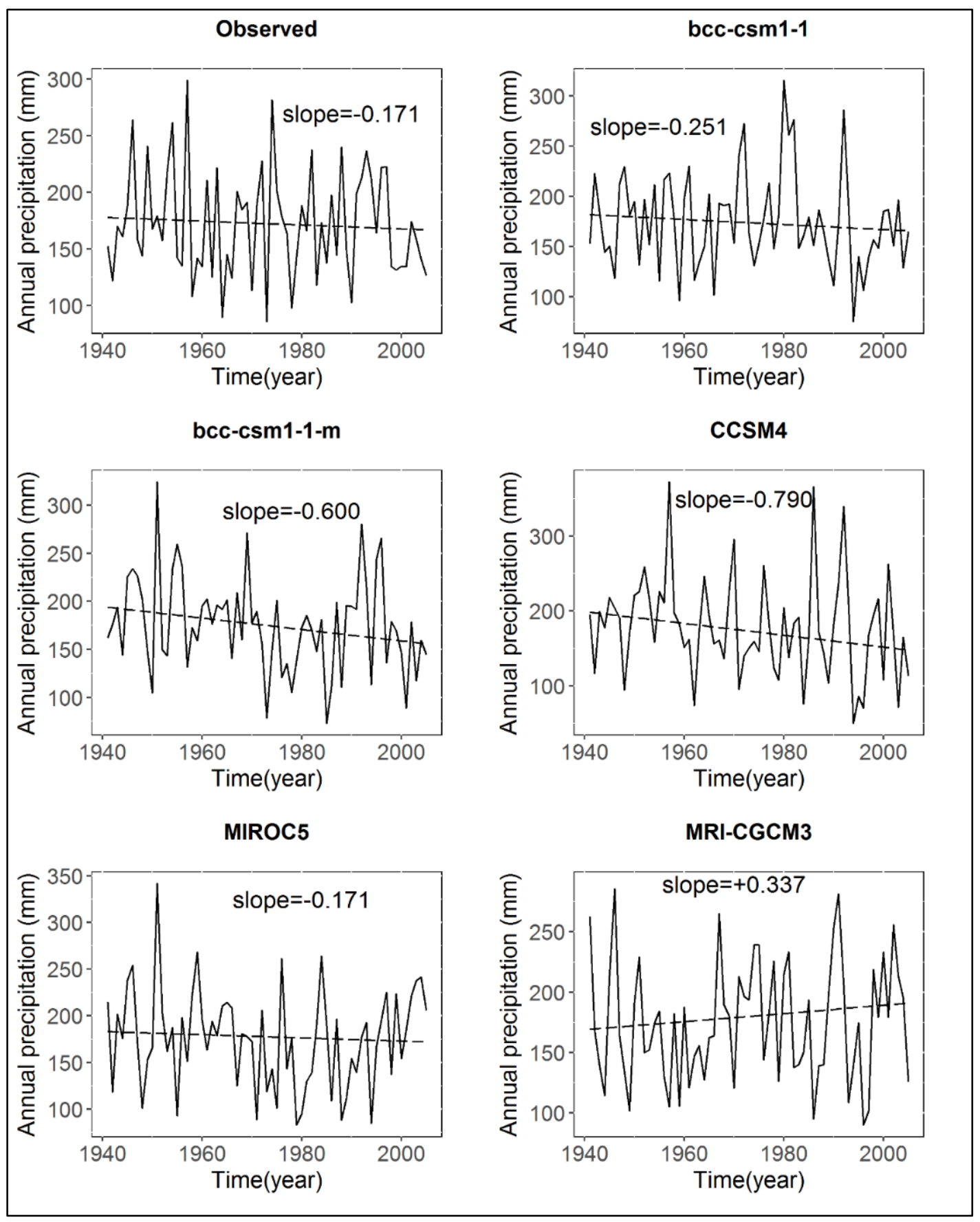

Fig. 8-12 Linear trends of the annual areal average rainfall for the observed and simulated time series using the Mann Kendall trend test over Iraq

The climate projection is the only source for the future climate data, and it's typically used with hydrological models for the long-term future prediction of water resources. Consequently, the trend of the precipitation from climate models for the long-term historical period is investigated and compared with observed one. This could be used 
to see whether climate models it can reproduce the historical climate condition. This assessment needs long historical records of observed and modelled precipitation. The different lengths of precipitation records affect the evaluation and conclusion of the trend quantification. However, for climate change impact studies, long-term records are needed to give a rough estimation of increasing and/or decreasing trend.

\subsection{Summary}

Accurate and reliable climate projections are the key to trustworthy and dependable climate change impact studies on hydrological processes and water resource planning and management. This chapter examines whether climate projections can reliably reproduce the observed trend of precipitation. Thirty stations data over 65 years have been studied over Iraq. A total number of 18 models from CMIP5 project with different spatial resolution are used to represent the typical projected climate data in this study. A non-parametric trend test Mann Kendall method is employed to test the trends in both datasets, followed by another analysis of the underlying probability distributions.

It can be concluded from the findings that the projected data of precipitation shows a persistently low performance with both substantial bias and very little correlation with the observed data. However, a certain agreement is also observed of the trend of annual precipitation regarding the direction (positive or negative) but not the value. This may occur due to the fact the rain itself is among the most difficult variables to simulate and its highly intermittent nature. Further, the preliminary analysis reveals that the observed appears to fit well with a beta type of probability distribution function PDF. For the modelled perception, 8 out of 18 models fit the same distribution.

It is also clear that there is a need to extend this study so that more projections from different climate models can be included. Further, the large-scale difference as to the use of coarse spatial resolution model data at catchment scale can be a significant source of errors, especially, when variables such as precipitation have a much higher 
spatial variability even within the catchment. In this regard, it may well be worth waiting until the resolution of climate models progress even finer to catchment scale, or probably more realistically, up-scaling the downstream model to reduce this scale gap to make the climate change impact study more reliable. Nevertheless, the findings of this study cast doubt over the practice of directly using projected precipitation for climate change impact study in hydrological processes. 


\section{Chapter 9 Climate Change Impact on Water Resources}

The primary objective of sustainable river basin management is to understand water resources systems and their types and relationships, e.g. groundwater, surface water, quantity and quality, biotic components, upstream and downstream interactions and human activities (physical flow control and water withdraw). Water resources management cannot be treated in separation; it is essential to consider with the performance of ecosystems simultaneously at different levels, and at different spatial and temporal scales. This often involves management and planning of water system at various local levels such as field, farm, and village and at regional levels such as catchments and river basins.

The first classification of IWRM problems is the technique of solving the interaction between surface and groundwater systems as solving both systems simultaneously (i.e. integrated or coupled models) or individually. Realistically, the major challenges of the selection of modelling technique are that whether it is capable and whether it can bridge the existing gaps in some models. The other classification is dependent on the complexity of solving the governing equations of the model, and they are allocation (decision-making software) and simulation models with or without climate change impact scenarios. From IWRM perspective, the modelling of highly-regulated river basins is very challenging because the flow is frequently interfered by human activities, such as water withdrawal among various users. For this reason, it is essential to consider the coupling of simulation and allocation models to investigate such interaction with climate change impact.

In this chapter, the calibrated SWAT model for the Dee River catchment (refer to chapter 3) is employed with climate data from the CMIP5 project (NCAR-CCSM4 model) with four future scenarios (RCP26, RCP45, RCP60 and RCP85) from 2006 to 2040. Then, the decision support tool, WEAP model (Water Evaluation and Planning software) is set-up, and the water supply data from SWAT model is used as the input for the river reach in the downstream of the Dee River. The coupling SWAT-WEAP is utilised to create various future scenarios of surface water abstraction of PWS in the 
downstream (Maximum licensed withdraw, $50 \%$ authorised abstractions and monthly time series with $1 \%$ annual increase of water use).

\subsection{Overview}

Climate change will probably influence on both surface and groundwater resources owing to the projected changes in evapotranspiration and rainfall and the spatial and temporal distribution of these key water balance components (Garner et al., 2017; Kirby et al., 2016). For instance, Trenberth (2011) pointed out that increasing intensities of rainfall will result in higher rates of surface runoff, decreased rates of groundwater recharge and an increased risk of flooding.

The impact of climate change on water resources needs to be quantified from regional to basin scales with the purpose of facilitating water resources planning and management to cope with future challenges. Global climate models (GCMs) are frequently utilised to grasp the climate dynamics and projecting future climate change. These models can offer input for climate change impact studies on coarse spatial resolution (typically 100-300 km). Nevertheless, it is still too coarse for any basin or regional scale of climate change impact studies.

The river basin (watershed) can be deemed as a suitable unit to perform analysis, planning of the challenges that confronting water resources. The looming climate change impact further worsens the situation with many studies showing that further climate can be more extreme, not only in the sense of more storms and flooding; it also means that the current arid areas will be subject to more severe droughts and water scarcity problems (Solomon, 2007). It is unsurprising that managing existing water resources has become a critical topic in many theoretical as well as practical studies (Jain and Singh, 2003).

In a broad sense, computer models are utilised to access reservoir operation, water allocation, flood risk assessment, drought conditions, groundwater development, water quality, irrigation operation and forecasting and control of high water. There are 
large numbers of software available to simulate problems water resources management, and it can be divided mainly into two groups, allocation (optimisation or decision-making system models) and simulation models (Loucks et al., 2005; Loucks, 2008; Condon and Maxwell, 2013).

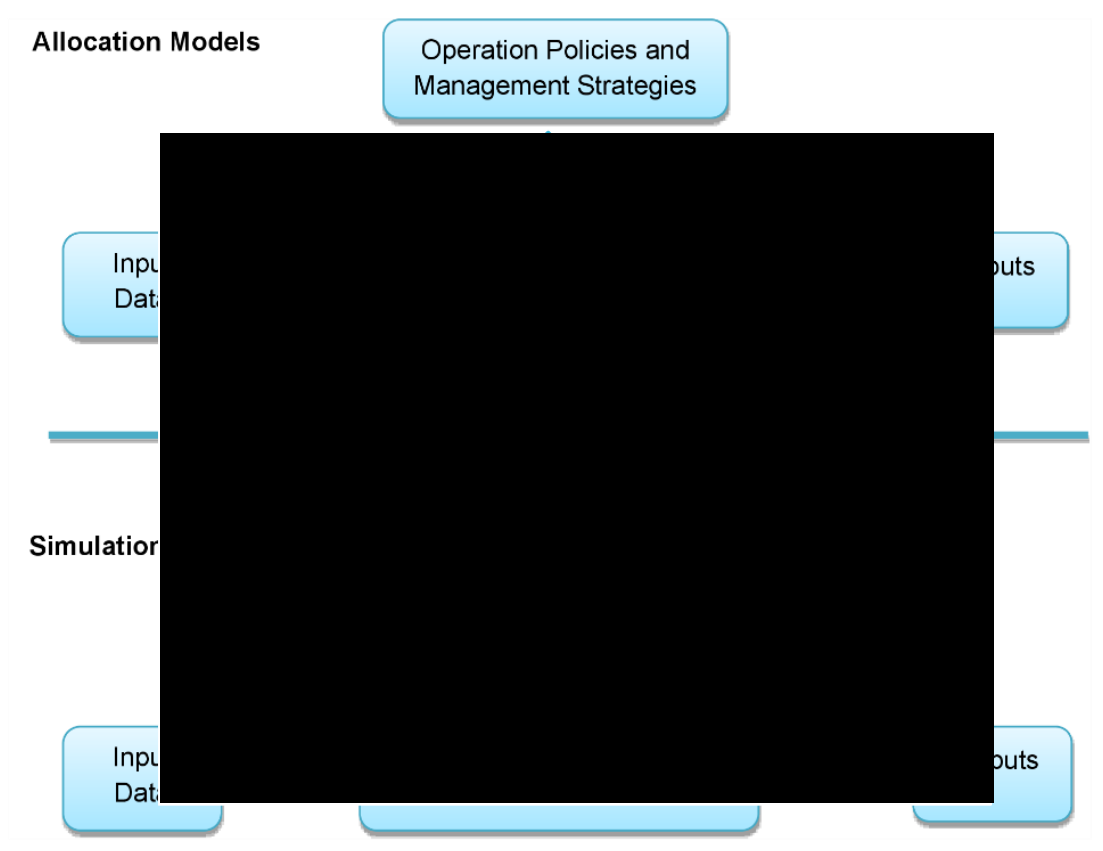

Fig.9-1 Management strategies within simulation and allocation models (After Loucks et al., 2005)

Simulation models address certain limitations of allocation models by solving physically based flow equations to offer spatially distributed water resources outputs for a number of parameters (runoff, water table elevation,...etc.) (Condon and Maxwell, 2013). Allocation (optimisation) models are frequently utilised in the applied problem of water resources management. These models optimise water allocation from various resources to meet a range of demands and what design and operating policy will best meet the identified objectives under a set of priorities and constraints (Loucks, 2008). The simulation and allocation models are discussed in chapter 2. Figure 9-1 demonstrates how the simulation and optimisation work with input, output data and operating policy and system design. 
The primary objective of this chapter is to evaluate the impacts of the likely future water use for public water supply on the water resources in the downstream area of the Dee River watershed. The calibrated SWAT model (from chapter 3) is used together with climate projections from the CMIP5 project (NCAR-CCSM4 model) for four future scenarios of (RCP26, RCP45, RCP60 and RCP85) from 2006 to 2040. The simulated streamflow of SWAT model with CMIP5 model data is used as the input to WEAP model to create different scenarios of water use rate of surface water abstraction for the public water supply PWS in Chester city.

For each future scenario, three scenarios of water use rate are considered: Maximum licensed abstraction, $50 \%$ of maximum licensed abstraction and time series with $1 \%$ annual increase of water use. Chester weir is utilised as a checkpoint of unmet flow requirement and coverage. The daily water uses in the UK has been gradually increasing by $1 \%$ per year since 1930, and the average person now consumes 150 litres a day (Waterwise, 2012).

\subsection{Methodology}

\subsubsection{Hydrological simulation}

SWAT model was created to simulate the hydrology of the basin, and the model was calibrated and validated based on historical daily streamflow at six sites (Catchment C, Fig. 3-6 in chapter 3, page 47), one location for both of Catchment A and B (Fig. 3-6). The main Dee River (Catchment C) was divided into 57 sub-basins to predict catchment hydrology. The CIMP5 climate data of NCAR-CCSM4 model (precipitation, maximum air temperature and minimum air temperature) are used with the calibrated SWAT model to simulate river flow for four future scenarios (RCP26, RCP45, RCP60 and RCP85) from 2006 to 2040 with a 2-years warm-up period to make hydrological cycle fully operational. 


\subsubsection{Water demand sites}

As mentioned before, the Dee River basin is an example of the complex river flow system and highly-regulated through a management scheme that provides water for both industrial and public water supply (PWS) in summers and prevents flooding between Bala lake and city of Chester in winters. There are massive PWS in the downstream area of the river basin (Chester city) as revealed in Figs. 9-2 and 9-3 which are considered for the evaluation of the impact of water demand on the availability of water resources under scenarios of climate change. These demands sites are:

1) PWS1 that consumes a maximum of $\left(686,446 \mathrm{~m}^{3} / \mathrm{day}\right)$;

2) PWS2 that consumes a maximum of $\left(686,446 \mathrm{~m}^{3} / \mathrm{day}\right)$;

3) PWS3 that consumes a maximum of $\left(36,000 \mathrm{~m}^{3} / \mathrm{day}\right)$; and

4) PWS4 that consumes a maximum of $\left(686,446 \mathrm{~m}^{3} / \mathrm{day}\right)$.

There are two key control points in the basin (DEFRA, 2014) as follows:

1) Chester Weir is a control point for the River Dee Regulation Scheme. The Dee Regulation Scheme aims to maintain a minimum of $\left(4.2 \mathrm{~m}^{3} / \mathrm{sec}\right.$.) under "Normal General Directions" at Chester Weir. The Chester Weir residual flow is calculated based on flows measured at the Chester Suspension Bridge ultrasonic flow gauge, minus the abstractions are taken by United Utilities from the Chester Weir intake.

2) Manley Hall gauging station is also a control point. It is our understanding that the Dee regulation Scheme aims to maintain around $\left(10.2 \mathrm{~m}^{3} / \mathrm{sec}\right.$.) at this point.

Chester weir is used to evaluate the unmet streamflow demand. 


$$
4
$$

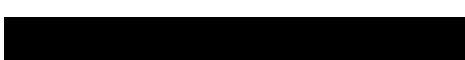

Fig. 9-2 Dee River demand sites (After Dee Valley Water and United Utilit 


\subsubsection{Future climate scenario}

In general, the UK climate is expected to become hotter and drier in the summer and warmer and wetter in the winter (DEFRA, 2008):

1. Average UK annual temperatures may rise by 2 to $3.5^{\circ} \mathrm{C}$ by the $2080 \mathrm{~s}$. In general, greater warming is expected in the South East than the North West of the UK, and there may be more warming in the summer and autumn than winter and spring. Under a 'High Emissions' scenario, the South East may be up to $50{ }^{\circ} \mathrm{C}$ warmer in the summer by the 2080s;

2. Annual average precipitation across the UK may decrease slightly, by between 0 and $15 \%$ by the 2080 s. But the seasonal distribution of precipitation will change significantly, with winters becoming wetter and summers drier. Under the 'High Emissions' scenario, precipitation in the 2080s may decrease in summer by $50 \%$ in the South East and an increase in winter by up to $30 \%$.

\subsubsection{WEAP model}

The Water Evaluation and Planning software WEAP is an integrated water resource management (IWRM) software that developed by the Stockholm Environment Institute SEI in the USA. It is designed to assess user-developed scenarios that accommodate changes in the socio-economic and biophysical conditions of catchments over time (Yates et al., 2005). WEAP allows planner access to a more comprehensive view of the broad range of factors that should be considered in managing water resources for present and future use owing to its integrated approach to simulating both the natural (e.g., runoff, baseflow, evapotranspirative demands, etc.) and engineered structures (e.g., reservoirs) of water resources systems (Sieber, 2018). 
WEAP operates in many capacities (Sieber, 2018) including:

1) Water balance database: WEAP provides a system for maintaining water demand and supply information;

2) Scenario generation tool: WEAP simulates water demand, supply, runoff, storage, pollution generation, treatment and discharge and instream water quality; and

3) Policy analysis tool: WEAP evaluates a full range of water development and management options, and takes account of multiple and competing uses of water systems.

WEAP has the high global popularity for the scenario analysis of water supply and demands and has been utilised in water-related issues/projects in different part of the world under effects of climate change (e.g. Bhave et al., 2018; Katirtzidou and Latinopoulos, 2017; Hao et al., 2015).

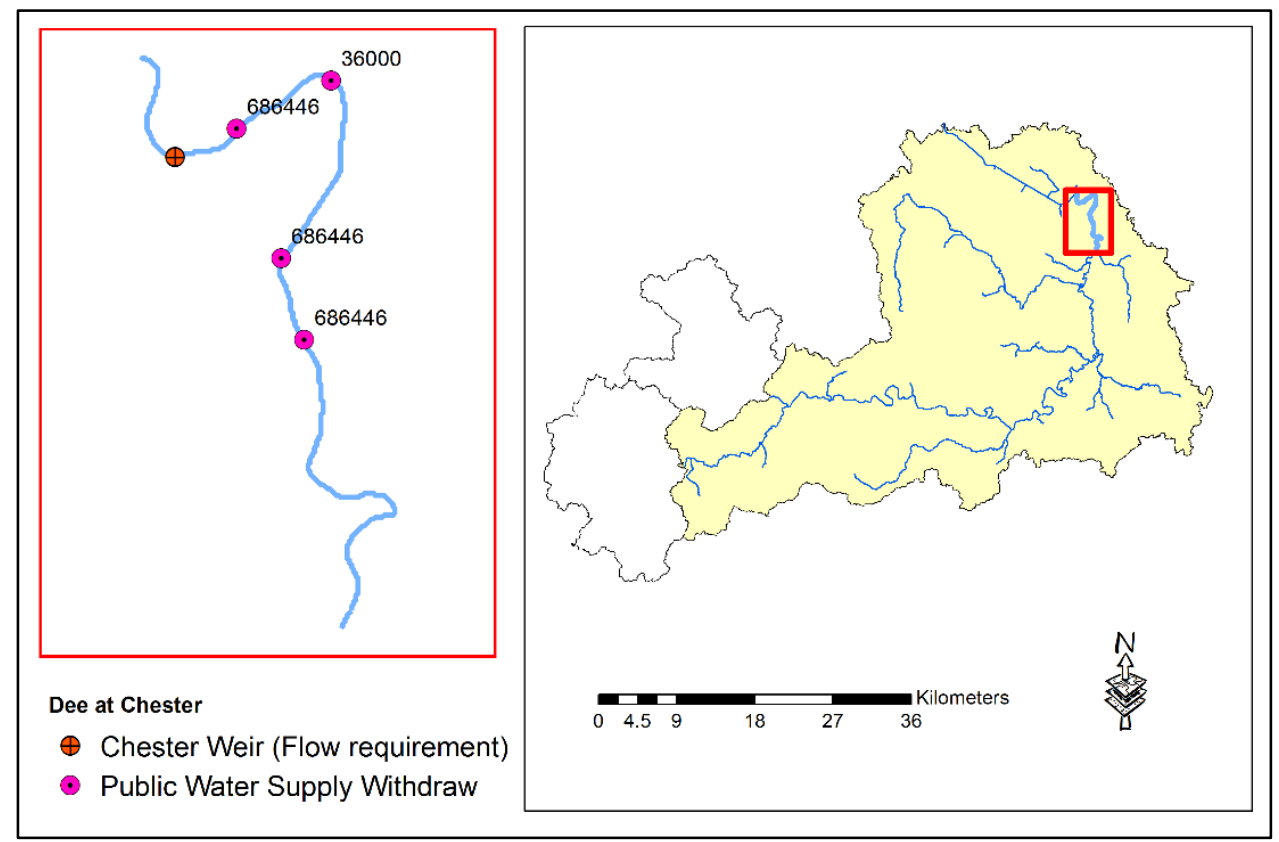

Fig. 9-3 Study region of coupled SWAT-WEAP model (surface water abstraction in $\mathrm{m}^{3} /$ day) 
The model then optimises water use in the basin using a linear optimisation algorithm to allocate water to the various demand sites, as per the demand priorities that range from 1 to 99 , with 1 being the highest priority. For more information on the WEAP model, readers are directed to (Yates et al., 2005; Sieber and Purkey, 2011). In the present study, the simulated streamflow for future scenarios of the SWAT model will be used as the input to the head of the river reach in the downstream to decision support tool, WEAP model. In the coupled SWAT-WEAP, Chester weir will be utilised as a checkpoint for minimum streamflow requirement for the ecological purposes with minimum river flow of $\left(4.2 \mathrm{~m}^{3} / \mathrm{sec}\right.$. $)$ and the evaluation of the unmet flow requirements and converge. Figure 9-3 shows the study area of the coupled SWAT-WEAP model.

The simulated discharge from the SWAT model is aggregated into monthly time series and utilised as input for the WEAP model. In the coupled SWAT-WEAP, 12 scenarios of surface water abstraction to check the unmet demands as follow:

1) $100 \%$ of the maximum licensed of surface water abstraction (RCP26 scenario, 2008-2040);

2) $50 \%$ of the maximum licensed of surface water abstraction (RCP26 scenario, 2008-2040);

3) Monthly time series (per cent of maximum licensed of surface water abstraction) (RCP26 scenario, 2008-2040) as it can be seen in Fig. 9-4;

4) $100 \%$ of the maximum licensed of surface water abstraction (RCP45 scenario, 2008-2040);

5) $50 \%$ of the maximum licensed of surface water abstraction (RCP45 scenario, 2008-2040);

6) Monthly time series (per cent of maximum licensed of surface water abstraction) (RCP45 scenario, 2008-2040);

7) $100 \%$ of the maximum licensed of surface water abstraction (RCP60 scenario, 2008-2040);

8) $50 \%$ of the maximum licensed of surface water abstraction (RCP60 scenario, 2008-2040); 
9) Monthly time series (per cent of maximum licensed of surface water abstraction) (RCP60 scenario, 2008-2040);

10) $100 \%$ of the maximum licensed of surface water abstraction (RCP85 scenario, 2008-2040);

11) $50 \%$ of the maximum licensed of surface water abstraction (RCP85 scenario, 2008-2040);

12) Monthly time series (per cent of maximum licensed of surface water abstraction) (RCP85 scenario, 2008-2040);

Figure 9-5 shows the flowchart of the coupled simulation model (SWAT), allocation model (WEAP) and the climate projection data (CMIP5 models).

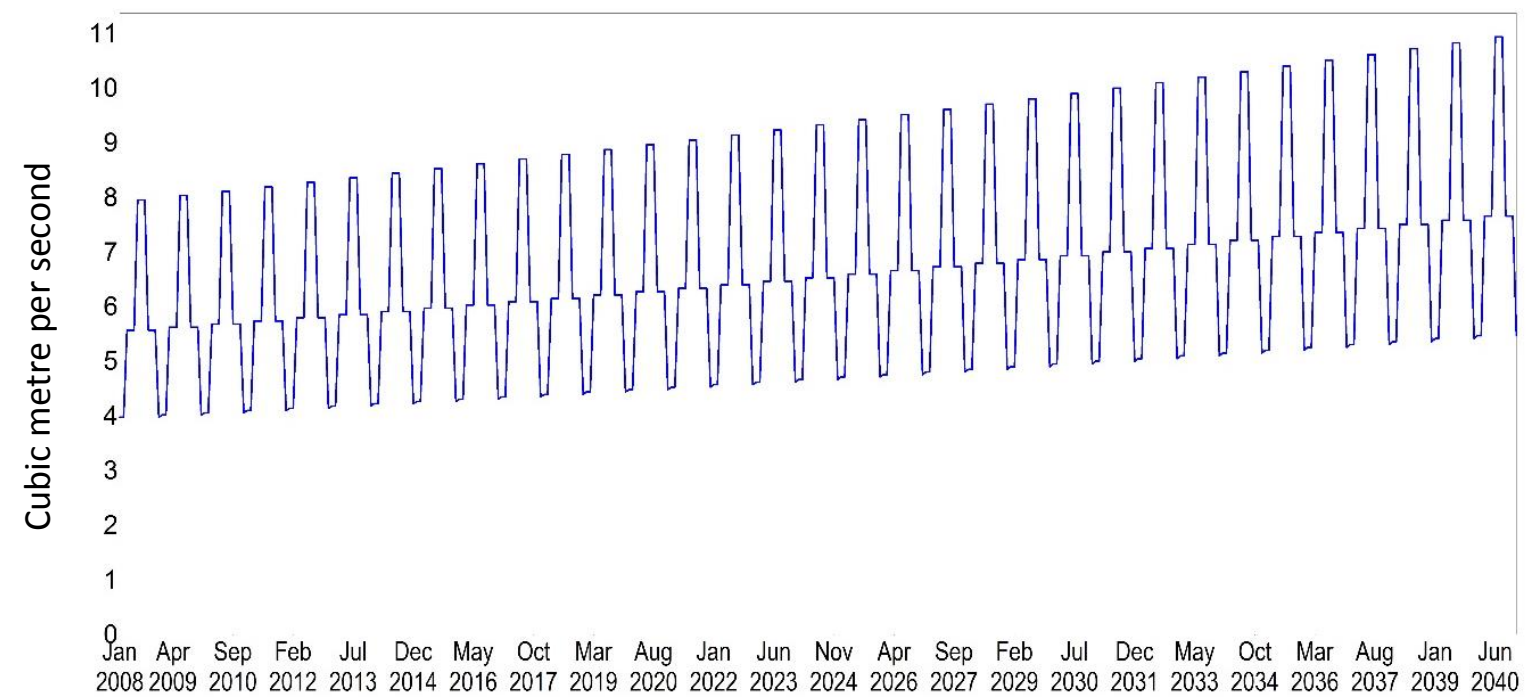

Fig. 9-4 Public water supply PWS1 abstractions for scenarios no. 3, 6, 9 and 12 [ $100 \%$ of maximum licensed abstractions for summer months (June, July and August), $50 \%$ of maximum licensed abstractions for winter months (December, January and February), and $70 \%$ for the rest with $1 \%$ increase of water use per year] 


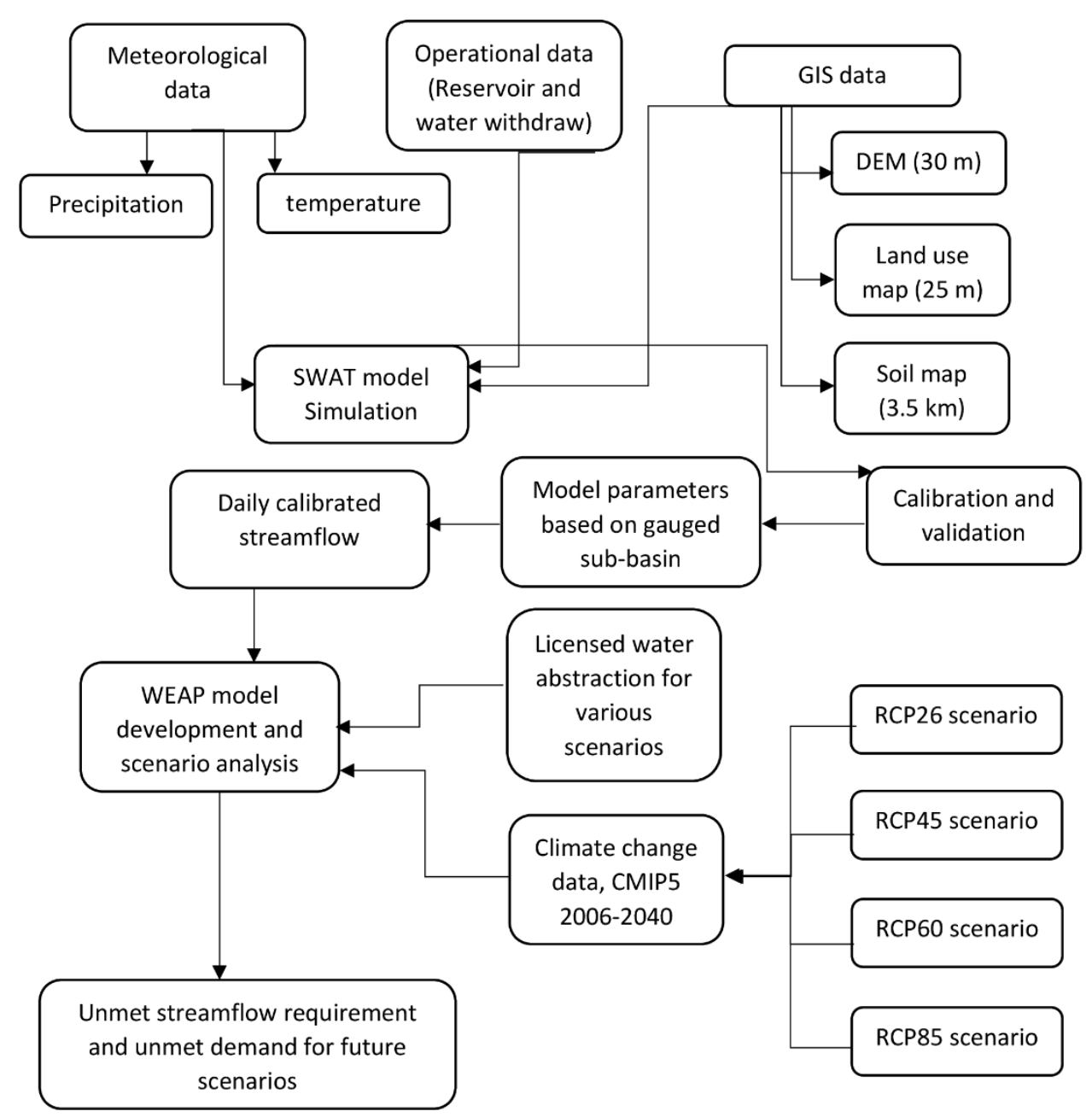

Fig. 9-5 Flowchart of the coupled SWAT-WEAP model framework

\subsection{Results and discussion}

The coupled SWAT-WEAP model is built for the future scenario to evaluate the likely unmet demands at the public water supply locations. Firstly, the unmet flow requirement and per cent of coverage at Chester weir station $\left(4.2 \mathrm{~m}^{3} / \mathrm{sec}\right.$.). Clearly, in the four emission scenarios, there is unmet flow demand from June until October with the maximum monthly average unmet flow of $1.8 \mathrm{~m}^{3} / \mathrm{s}$ (57\% of flow needed) in August for the RCP26 scenario as illustrated in Fig. 9-6 and 9-7 and tables 9-1 and 9-2. 


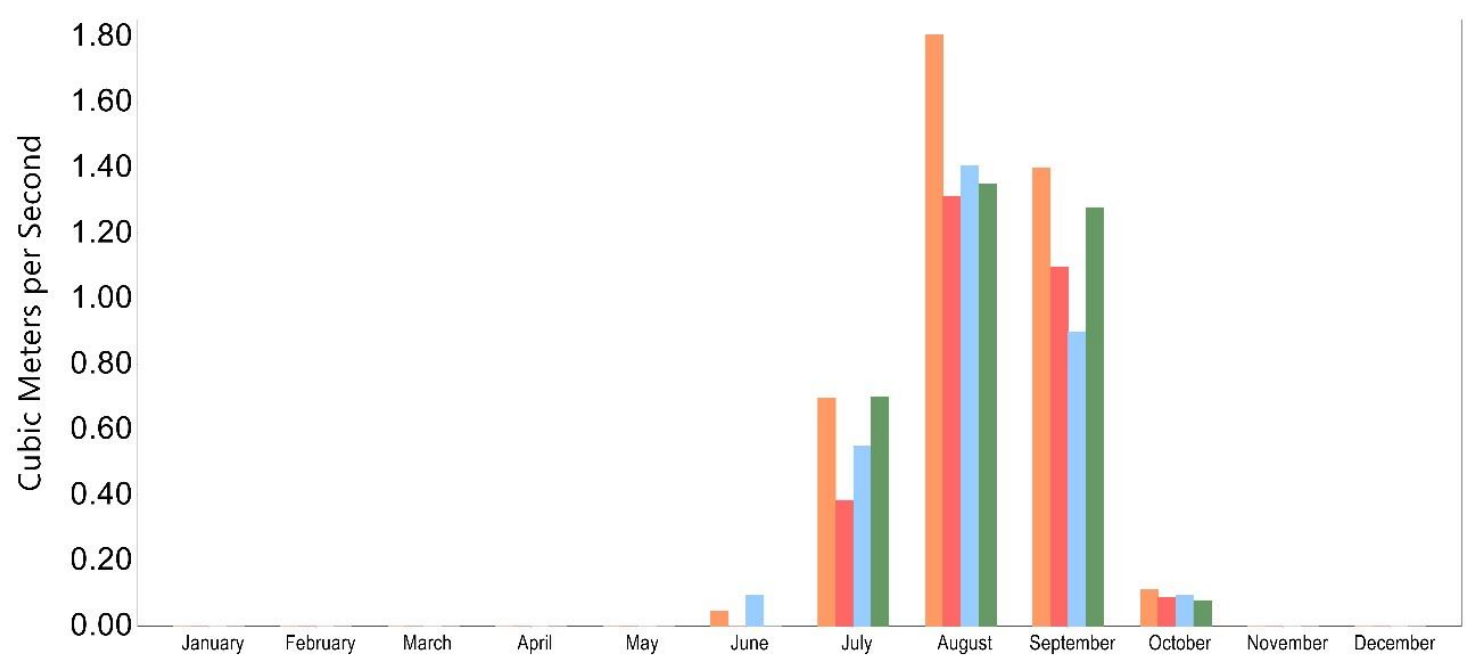

Fig. 9-6 The average monthly unmet streamflow requirement $\left(\mathrm{m}^{3} / \mathrm{sec}\right.$.) in Chester weir for the four future scenarios for the period of 2008-2040

Table 9-1: The average monthly unmet streamflow requirement $\left(\mathrm{m}^{3} / \mathrm{sec}\right.$.) in Chester weir for the four future scenarios for the period of 2008-2040

\begin{tabular}{c|ccccc}
\multicolumn{2}{c}{ June } & July & August & September & October \\
\hline RCP26 & 0.047 & 0.697 & 1.807 & 1.401 & 0.112 \\
RCP45 & 0.000 & 0.385 & 1.314 & 1.097 & 0.088 \\
RCP60 & 0.096 & 0.550 & 1.407 & 0.900 & 0.094 \\
RCP85 & 0.000 & 0.700 & 1.350 & 1.280 & 0.079
\end{tabular}




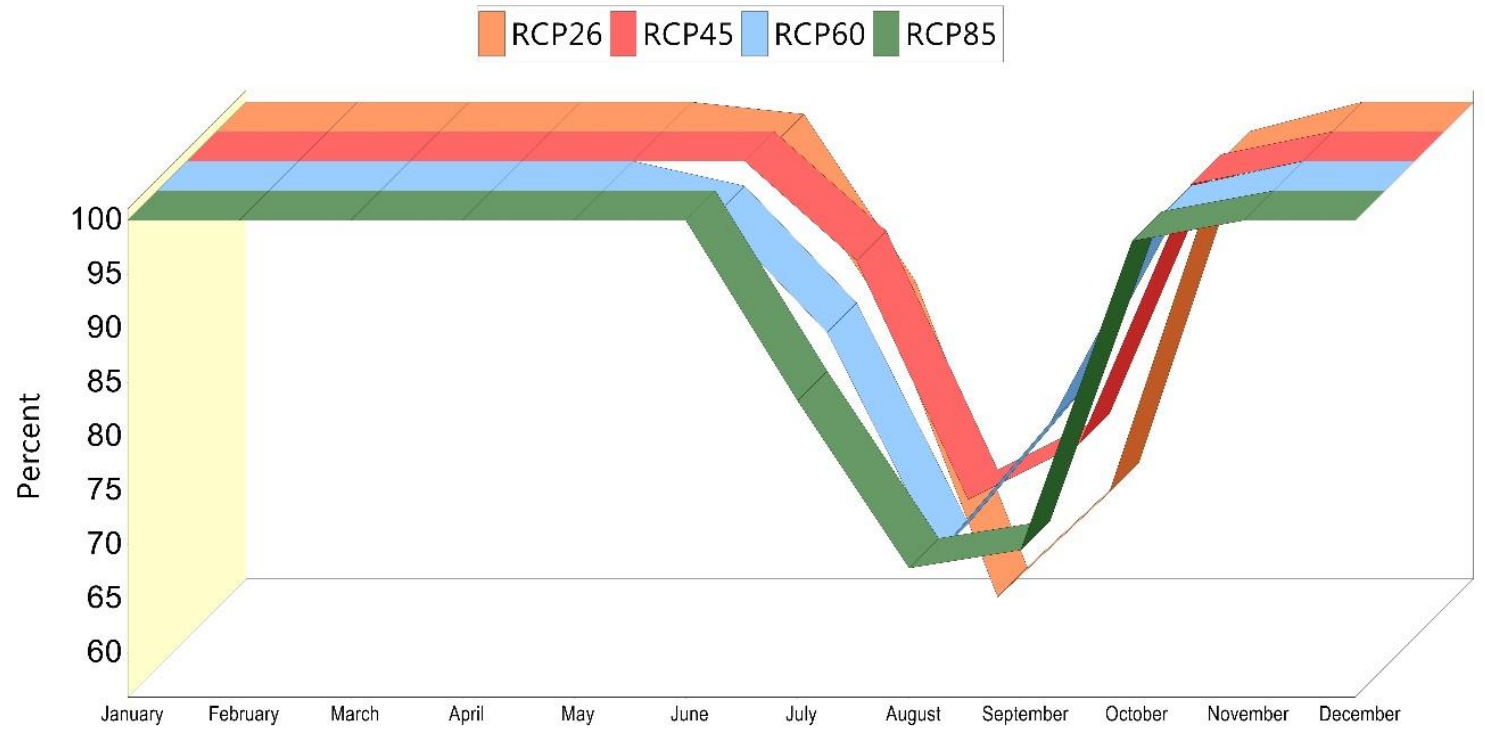

Fig. 9-7 The average monthly flow requirement coverage (\% of flow requirement) in Chester weir for the four future scenarios for the period of 2008-2040

Table 9-2: The average monthly flow requirement coverage (\% of flow requirement) in Chester weir for the four future scenarios for the period of 2008-2040

\begin{tabular}{c|ccccc}
\multicolumn{2}{c}{ June } & July & August & September & October \\
\hline RCP26 & 98.9 & 83.4 & 57.0 & 66.7 & 97.3 \\
RCP45 & 100.0 & 90.8 & 68.7 & 73.9 & 97.9 \\
RCP60 & 97.7 & 86.9 & 66.5 & 78.6 & 97.8 \\
RCP85 & 100.0 & 83.3 & 67.8 & 69.5 & 98.1
\end{tabular}

The average monthly unmet demand for maximum surface water abstraction is revealed in Fig. 9-8. It can be clearly seen that in the summer a significant unmet demand reaches more than $4 \mathrm{~m}^{3} / \mathrm{sec}$ for the PWS1, PWS2 and PWS4 as in Fig. 9-8a for all of the future scenarios. On the other hand, PWS3 has also had unmet demand with a maximum projected value of $0.04 \mathrm{~m}^{3} / \mathrm{sec}$. 
\begin{tabular}{ll|l|l} 
RCP26 & RCP45 & RCP60 & RCP85
\end{tabular}

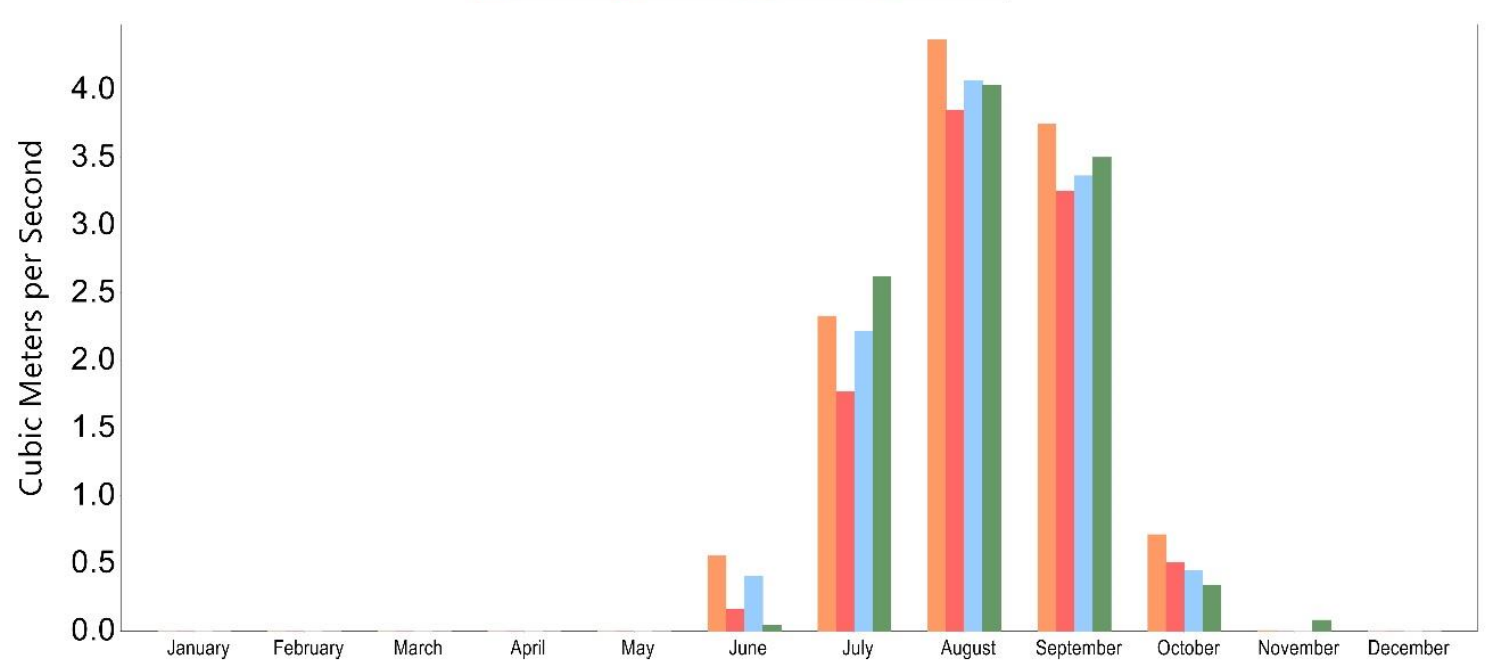

a. PWS1, PWS2 and PWS4

\begin{tabular}{l|l|l|l} 
RCP26 & RCP45 & RCP60 & RCP85
\end{tabular}

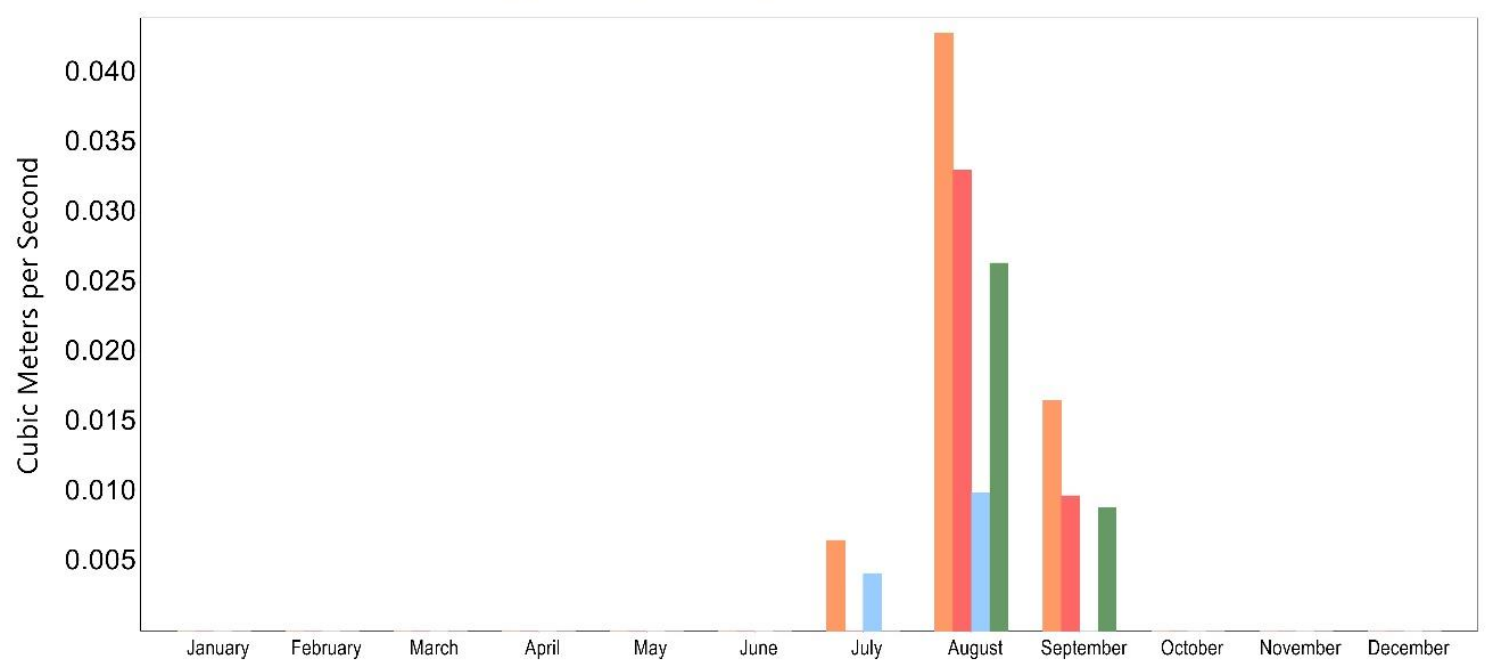

b. PWS3

Fig. 9-8 The average monthly unmet demand $\left(\mathrm{m}^{3} / \mathrm{sec}\right.$.) for public water supply PWS for the four future scenarios for the period of 2008-2040 for the maximum withdraw scenario

Similarly, the unmet demand for $50 \%$ of maximum water withdraw and time series water uses scenarios are presented in Fig. 9-9 and 9-10. Again, summer months have a tremendous value of unmet demand. 


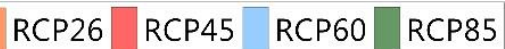

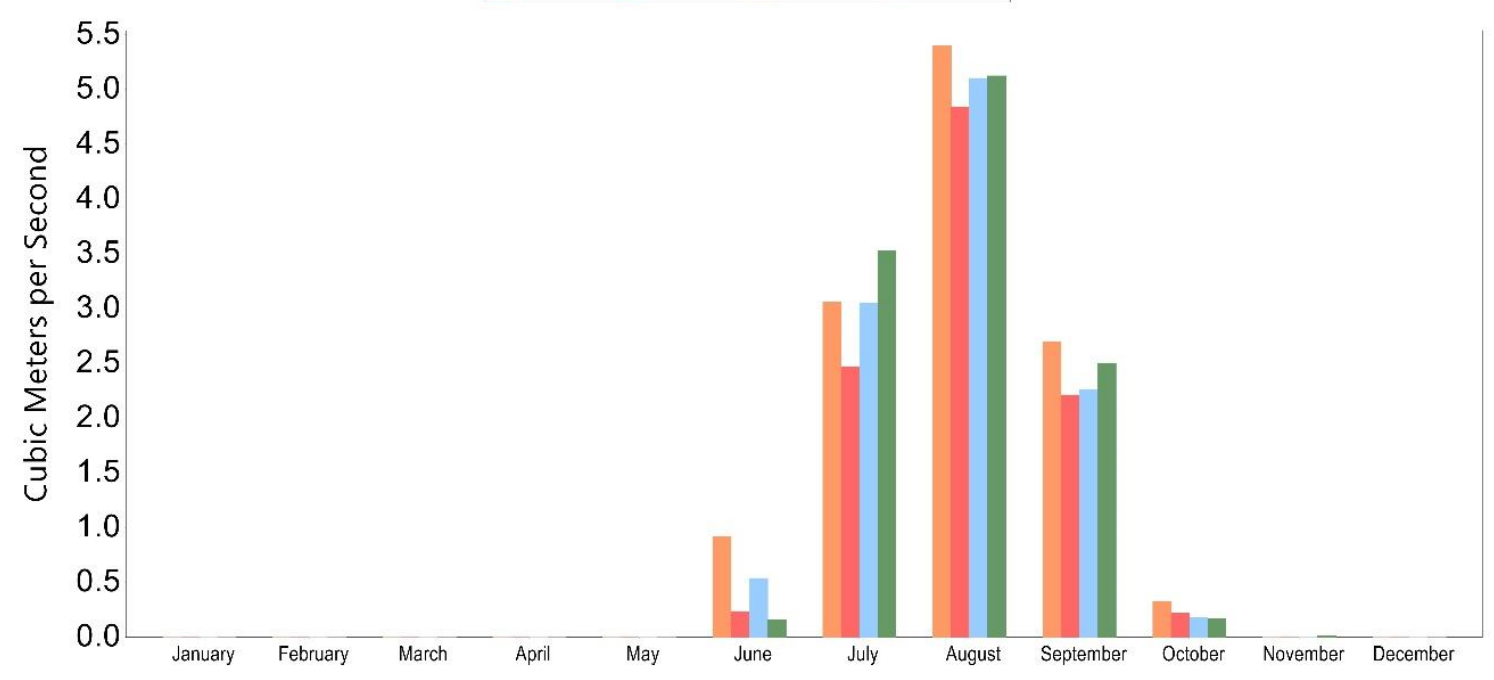

a. PWS1, PWS2 and PWS4

\begin{tabular}{l|l|l|l|} 
RCP26 & RCP45 & RCP60 & RCP85
\end{tabular}

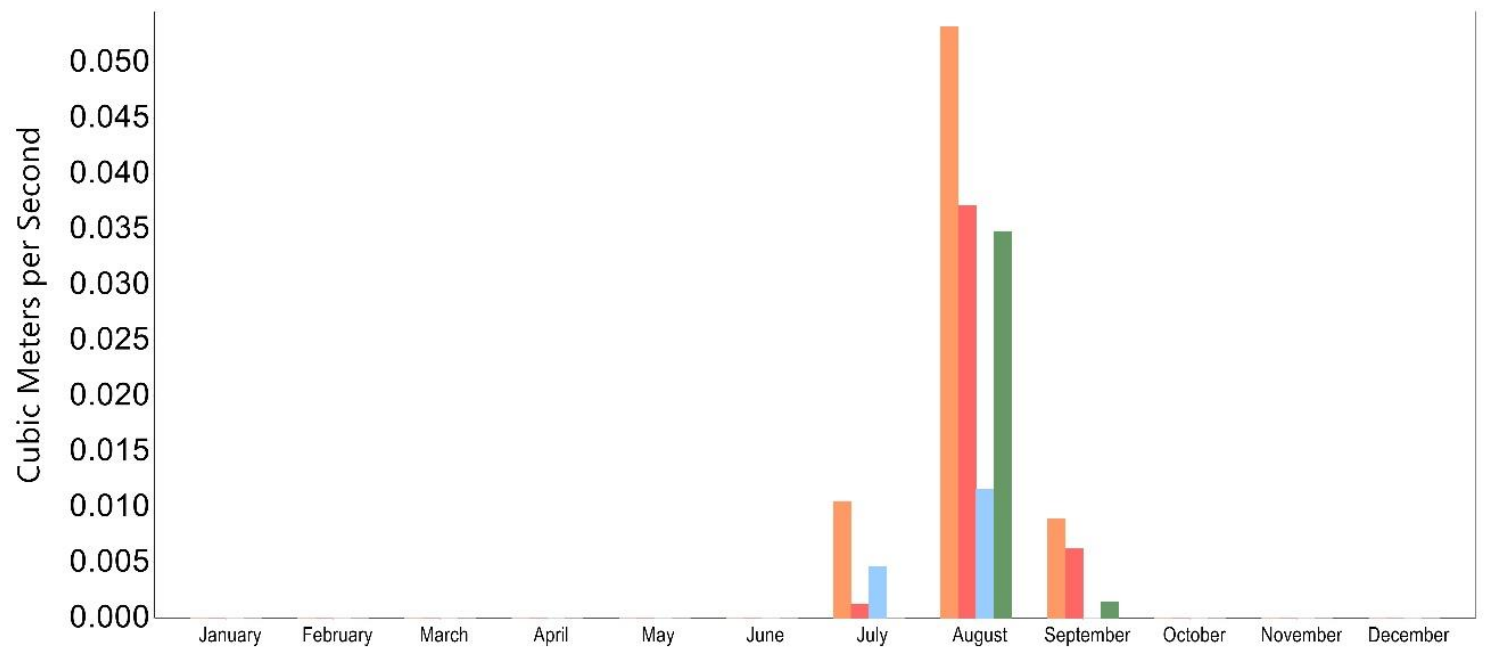

b. PWS3

Fig. 9-9 The average monthly unmet demand $\left(\mathrm{m}^{3} / \mathrm{sec}\right.$.) for public water supply PWS for the four future scenarios for the period of 2008-2040 for the $50 \%$ maximum withdraw scenario 
\begin{tabular}{ll|l|l|} 
RCP26 & RCP45 & RCP60 & RCP85
\end{tabular}

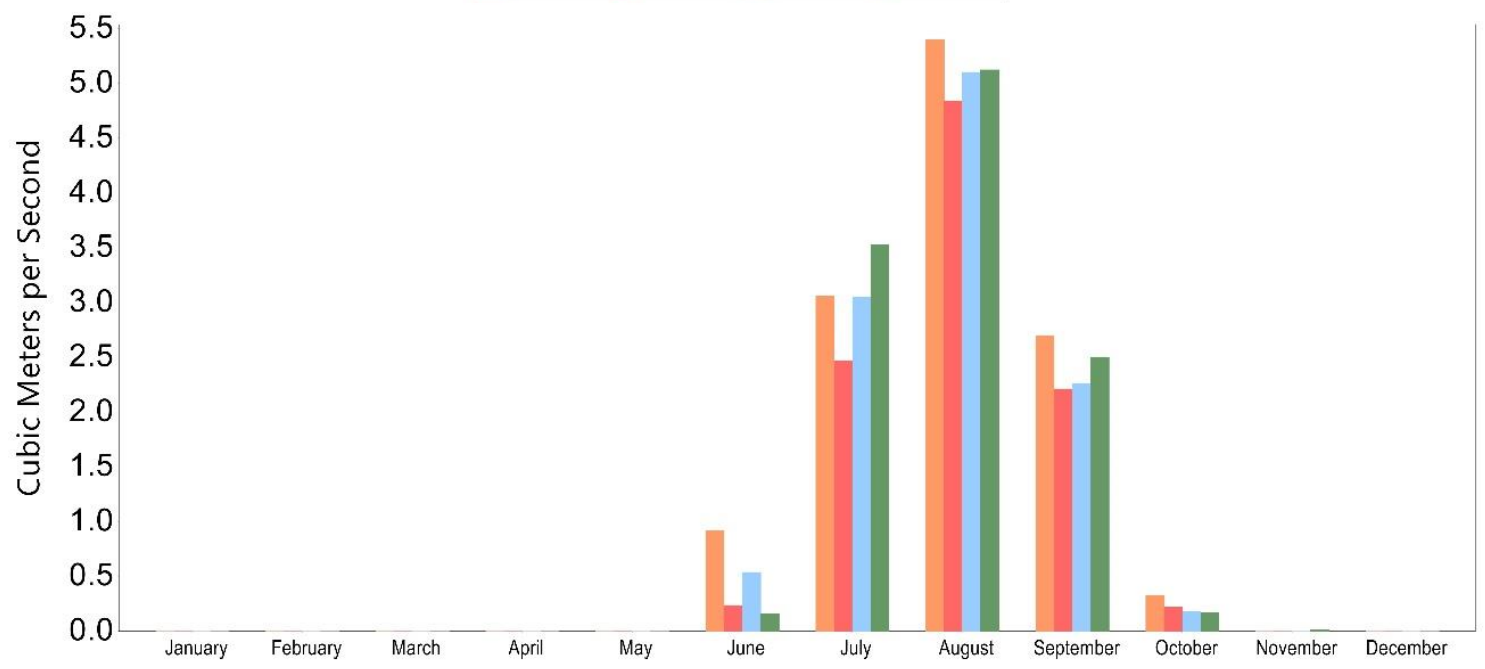

a. PWS1, PWS2 and PWS4

\begin{tabular}{ll|l|l} 
RCP26 & RCP45 & RCP60 & RCP85
\end{tabular}

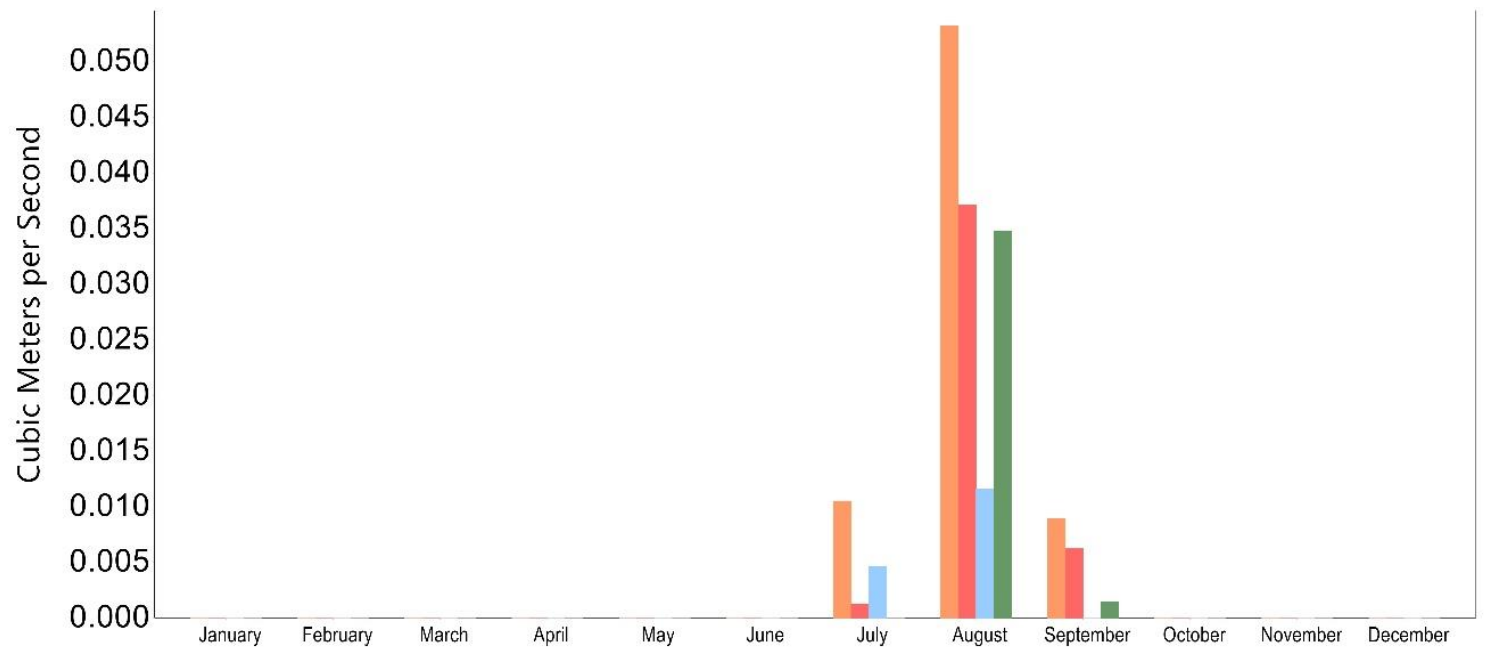

b. PWS3

Fig. 9-10 The average monthly unmet demand $\left(\mathrm{m}^{3} / \mathrm{sec}\right.$.) for public water supply PWS for the four future scenarios for the period of 2008-2040 for the time series withdraw scenario 


\subsection{Summary}

In this chapter, the coupled SWAT-WEAP model is setup and used in the highlyregulated river basin with climate change data of CMIP5 project to evaluate unmet demand of water for public water supply in the downstream region (city of Chester) as well as check unmet streamflow requirement in Chester weir. The SWAT model was previously calibrated against historical daily streamflow, afterward, the future climate data of NCAR-CCSM4 model precipitation, maximum and minimum air temperature), part of CMIP5, (for four future scenarios: RCP26, RCP45, RCP60 and RCP85) are used as input in calibrated SWAT model to simulate the catchment hydrology for the period of 2006-2040 with 2 years' warm-up period. The simulated discharge from the SWAT model will be used as input to the modelled reach in WEAP model on a monthly time step.

Twelve scenarios of water use rate of 4 selected location of public water supplies in the downstream of the Dee River basin with a considerable amount of water abstraction utilised to evaluate the likely unmet demands. The results agreed that there is expected unmet demand with large quantity, especially in the summer season (June, July and August). Actions and measurements for mitigating the effects of unmet water demands and uncertainties as to how the climate will change and how it will affect water resources are the challenges that designers and planners will have to cope. How water resources management will have to adapt to climate changes is the pressing question to be answered. The possible mitigations for the unmet water demands are:

1) Augmenting streamflow from a deep well source;

2) Using reclaimed water;

3) Storing and recovering surface or groundwater;

4) Transferring water into basins; and

5) Adjust reservoirs regulation rules. 


\section{Chapter 10: Conclusions and Recommendations}

This chapter concludes this study and presents some recommendations for future work.

\subsection{Main conclusion}

A hydrological model for a highly-regulated watershed, the Dee River basin in the United Kingdom was developed using SWAT model to examine the human interventions within natural hydrological systems within SWAT through creating different scenarios regarding reservoirs releases and made a rough estimation of daily water withdraw within the study area. This study also investigated the impact of model input uncertainty, i.e. rainfall on the model output, i.e. streamflow and examined the climate change impact (i.e. the trend of likely drought and likely flooding) using quantile regression method on the observed variables, i.e. precipitation and simulated results of streamflow and catchment water yield. The model was thoroughly calibrated and validated against measured daily streamflow at eight river gauges stations.

Additionally, the SWAT model is coupled with a groundwater flow model, MODFLOW to improve the baseflow estimation of standalone SWAT model. The model uncertainty of the coupled SWAT-MODFLOW is scrutinised using the SAFE toolbox. As climate change data is uncertain, the study examines climate change data from the CMIP5 project to check whether the simulated rainfall can produce the trend of observed precipitation. Moreover, the SWAT model is coupled with water allocation model, WEAP to study considers several possible future climate conditions regarding the water use rate for the public water supply in the downstream of the Dee River basin. 
There were four main parts of this study: the first part is the modelling of highlyregulated river system, the Dee River watershed, by incorporating the complex hydrological processes, regulatory mechanisms and the drivers of changes at a primarily daily temporal scale and at spatial scales from the sub-catchment scale to the basin scale. The Dee River basin is managed thoroughly via the physical flow control and storage structures (i.e. reservoirs in the upstream) and a range of water sharing rules and regulations that provide resources for a variety of water needs: public water supply and industrial consumptions in the downstream part of the study basin. An approximate approach was utilised to calculate the daily water abstraction at alongside the river basin, as this amount is not available from the data collected. The calculation was based on the information from public water supplies companies who provide a weekly abstraction forecast to Natural Resources Wales for the assistance in calculating the required releases from the reservoirs. Justifiably, this method, although aids to reveal the interaction between streamflow regulation and surface water abstraction.

The second part is related to model input uncertainty through the investigation of the impact of several areal precipitation pre-processing methods on the hydrological model performance of Dee River basin. The latest high-resolution gridded precipitation dataset CEH-GEAR is used to quantify such implications for calibration and validation of a quasi-distributed model SWAT. The associated term 'compensation' owing to model parameterisation was also investigated by comparing the three distinctive models calibrated with different rainfall pre-processing methods: the centroid point estimate method (CPEM), the grid area method (GAM) and the grid point method (GPM). The models were further cross-validated over the different period to separate the changes in performances caused by model calibration and the input precipitation from different preprocessing methods. 
The third part is the use of the quantile regression technique (Linear and non-linear) for analysing the trend in climatic variables. The long-term precipitation data from two drastically different climate regions is examined focusing on the trends of the data close to 'extreme' regimes, to link them to the events of interests. The quantile regression technique is also combined with several extreme and drought indices to investigate the long-term trend. The linear quantile regression technique is also combined with the SWAT model to examine long-term trends of simulated river discharge and catchment water yields under the impact of water resources management practice. Three daily SWAT models are set up to simulate the hydrological processes in the Dee River catchment in the UK with flow control and water withdrawal process explicitly represented. Two scenarios (with and without flow control) are constructed to explore the impact of management practice. Further, two quantiles 0.02 and 0.98 are used to signpost high flow (wet) and low flow (dry) conditions that water managers are typically concerned with. The quantile of 0.5 is also considered as the medium flow and water yield.

The fourth part is the coupling SWAT model with MODFLOW to improve the simulation of baseflow as this variable concern the decision makers for water resources management. Moreover, parameters uncertainty for the SWAT model and the coupled SWAT-MODFLOW are scrutinised using SAFE toolbox within MATLAB environment. The dotty plots, Generalized Likelihood Uncertainty Estimation GLUE and Elementary Effect Test EET are used to quantify parameters uncertainty.

The primary findings of this thesis are as follows:

1) For the modelling of highly-regulated river basin: the demonstration of the management rules regulations such as flow control and water withdraw in the modelling process within the study area is capable of revealing the impact from 
the latter. It lays a foundation for further study on how various management practice can mitigate the effect of other sources such as climate change on catchment water resources management. An estimated method was used to estimate the daily water withdrawal amount at the water abstraction points. This approach, while helps to show the interaction between flow regulation and water abstraction.

2) For Model input uncertainty:

a) The results show the GEH-GEAR dataset is consistent with the rain gauge measurements and can be a trustworthy source for model calibration and validation. Additionally, both GPM and GAM methods are theoretically better than the default CPEM used by SWAT, and they also support to improve the calibration and validation of the model;

b) While model calibration varies when using different pre-processed precipitation data, it is the rainfall input data that controls the crossvalidation performance rather than how a model is calibrated;

c) A less well-calibrated model due to the use of a low-grade pre-processing method such as CPEM can do equally well when fed with better-preprocessed precipitation data such as GAM or GPM during validation;

d) A model earlier calibrated with low-quality rainfall data can still use highquality rainfall inputs when they become available at later times without having to re-calibrating which is often limited by the length of data.

3) For the use of quantile regression technique:

a) The QR based trend analysis can offer far more detailed information with respect to the quantity in question, and this is principally valuable for water managers who are more worried about extreme values instead of the average one;

b) The QR method can help form a comprehensive picture of climatic regarding their variation over time at different frequency and magnitude;

c) The association of quantile gets an extra benefit to bridge the trend analysis with a frequency that indicates an excessive potential of its use in 
studying climate change impact on engineering design without the existence of limitation by assumptions of data stationarity;

d) The QR helps better to grasp the climate change impact. A decreasing trend in summer precipitation might still be accompanied with increasing severe storms in the same season;

e) The combination of quantile regression and semi-distributed hydrological modelling approach prefers in presenting spatially distributed trend information for extremely dry and wet scenarios, which can thoroughly address the needs of practitioners and decision makers in dealing with long-term planning of water resources under climate change;

f) The demonstration of the management practice such as physical flow control and water withdraw in the modelling process can reveal the impact from the latter, and as such lays a foundation for further study on how numerous management practices can mitigate the impact from other sources such as climate change on catchment water resources management;

g) It has been shown that the 0.98 quantile has an increasing trend for both 'real-life' and natural flow scenario with a significant trend in most of the river basin. Furthermore, the result shows that the management practices tend to decrease the floods in the catchment.

h) For the 0.02 quantile, both positive (mainly upstream) and negative (downstream) trends exist for the two scenarios, although most of them are statistically insignificant. The comparison of the two scenarios indicates that the management practices are undoubtedly able to mitigate strong decrease trends in the downstream;

i) The key finding is that trends of low quantile 0.02 are mostly insignificant necessitates further study. As the trend analysis is carried out over the simulated data, the performance of the model, particularly its representativeness of high and low flow conditions may directly affect the results hence the conclusion; 
4) The coupled SWAT-MODFLOW are revealed to simulate the hydrological processes in the Dee River basin with focus on the baseflow simulation. The results show that the coupled models can produce comparably better simulations of low flows in the stream network, and thus improved the water balance in the catchment. Generally, the study shows an encouraging direction for using coupled surface-groundwater model in the concept of IWRM.

5) This study has also evaluated the possibility of two hydrological models that have a difference in the depiction of processes that are associated with groundwater flow modules such as spatial distribution of groundwater level and hydraulic conductivity, and are assessed for their performance to simulate streamflow with a reduced amount of uncertainty. The SWAT model (semi-distributed which has lumped groundwater module) and the coupled SWAT-MODFLOW (fullydistributed hydrological model) are applied over Alyn catchment from 1995 to 2000. Parameter uncertainty is examined using the EET and GLUE methods both the SWAT model and the coupled SWAT-MODFLOW. It can objectively highlight the following:

a) Model sensitivities and uncertainties towards the input parameters are the key base of model development and improvement which can solve the problems associated with water resources planning and management;

b) Since parameter uncertainty was only capable of quantifying a small part of whole uncertainty in the models, this study suggests further studies prerequisite on model structure.

c) The term parameters 'equifinality' exhibited there is no unique parameter estimation for some parameters in this study, and therefore uncertainty in the appraised parameters in the study basin is recognisable. This result agreed well with some other studies as aforementioned previously.

d) The likely reason for 'equifinality' is because parameters obtained from the calibration process were influenced by some other causes such as sensitivity or insensitivity in parameters and correlations amongst parameters. 
6) For the climate projections: it can be objectively concluded from that the projected data of precipitation shows a persistently low performance with both substantial bias and very little correlation with the observed data. However, a certain agreement is also observed of the trend of annual precipitation regarding the direction (positive or negative) but not the value. This might be owing to the fact the precipitation itself is among the most difficult variables to simulate and its highly intermittent nature. Nevertheless, it is still the only source of future climate data.

\subsection{Recommendations}

Indeed, more studies are needed to improve the method with respect to the problems found in this study, which include but are not limited to:

1) The technique used to estimate the daily surface water abstraction with the SWAT model presented some uncertainties. It can be developed by further modelling the probabilistic nature of water abstraction or by conditioning them on other variables such as temperature.

2) For quantile regression:

a) A better link between quantiles and event frequency (return period) in the context of trend analysis;

b) The quantiles used in this study are not really 'extreme'. Reliable quantile regression for the higher quantile (e.g., 0.99) needs to be examined; and

c) The method needs to be refined further to be more reliable on lower quantiles. It has been found that most trends of quantile 0.02 are insignificant, but this may not be true as the lower end of the data suffers from more significant errors, and zero values may as well interfere with the process.

3) For the uncertainty analysis of the coupled SWAT-MODFLOW: 
a) Because of a long time of the coupled SWAT-MODFLOW, only 1,000 simulations is considered. Therefore, it is suggested to create more runs with 10,000 and larger;

b) Only one ranking and screening method are used (EET method) to rank the most sensitive parameters. More techniques of ranking and screening might utilise for the comparison; and

c) More parameters need to be combined and considered with GLUE and different screening methods for both the SWAT and the coupled SWAT-MODFLOW.

4) For climate projection data: It is also clear that there is a need to extend this study to large-scale applications, i.e. global or continental scale. Further, the large-scale difference as to the use of coarse spatial resolution model data at catchment scale can be a key source of errors, especially, when variables such as precipitation have a much higher spatial variability even within the catchment. In this regard, it may well be worth waiting until the resolution of climate models progress even finer to catchment scale, or probably more realistically, up-scaling the downstream model to reduce this scale gap to make the climate change impact study more reliable. However, the findings cast distrust over the practice of directly using projected precipitation for climate change impact study in hydrological processes.

5) Since land use change has a significant influence on the hydrologic characteristics of soil and water management in a watershed, the potential impacts of the land use need to be investigated on the hydrological response (e.g. streamflow).

6) The groundwater flow model was constructed with a single layer unconfined aquifer (taking average of the two layers) and with one stress period (i.e. groundwater abstractions are assumed to be constant during the period of the simulation) to simplifying modelling processes and reducing the running time of the coupled SWAT-MODFLOW. A multiple stress 
Chapter 10: Conclusions and Recommendations

periods/layers scenario might be worthy to try examine how these settings influence on the estimations of the streamflow and the baseflow. 


\section{References}

Abbaspour, K., 2013. SWAT-CUP 2012: SWAT calibration and uncertainty programs-a user manual. Eawag: Dübendorf, Switzerland, 103.

Abbaspour, K., Faramarzi, M., Ghasemi, S., and Yang, H., 2009. Assessing the Impact of Climate Change on Water Resources in Iran. Water Resources Research, 45(10), n/a-n/a.

Abbaspour, K., Johnson, C., and Van Genuchten, M., 2004. Estimating Uncertain Flow and Transport Parameters Using a Sequential Uncertainty Fitting Procedure. Vadose Zone Journal, 3(4), pp.1340-1352.

Abbaspour, K., Rouholahnejad, E., Vaghefi, S., Srinivasan, R., Yang, H., and Kløve, B, 2015. A Continental-Scale Hydrology and Water Quality Model for Europe: Calibration and Uncertainty of a High-Resolution Large-Scale SWAT Model. Journal of Hydrology, 524, pp.733-752.

Abbaspour, K., Yang, J., Maximov, I., Siber, R., Bogner, K., and Mieleitner, J. et al., 2007. Modelling Hydrology and Water Quality in the Pre-alpine/alpine Thur Watershed using SWAT. Journal of Hydrology, 333(2-4), pp.413-430.

Abbott, M., Bathurst, J., Cunge, J., O'Connell, P., and Rasmussen, J., 1986. An Introduction to the European Hydrological System - Systeme Hydrologique Europeen, "SHE", 2: Structure of a Physically-based, Distributed Modelling System. Journal of Hydrology, 87(1-2), pp.61-77.

Abdo, K. S., Fiseha, B. M., Rientjes, T. H. M., Gieske, A. S. M., and Haile, A. T., 2009. Assessment of Climate Change Impacts on the Hydrology of Gilgel Abay Catchment in Lake Tana Basin, Ethiopia. Hydrological Processes, 23(26), pp.36613669.

Aeschbach-Hertig, W. and Gleeson, T., 2012. Regional strategies for the accelerating global problem of groundwater depletion. Nature Geoscience, 5(12), p.853. 
Agyenim, J.B. and Gupta, J., 2012. IWRM and developing countries: Implementation challenges in Ghana. Physics and Chemistry of the Earth, Parts $A / B / C, 47$, pp.4657.

Ahl, R.S., Woods, S.W. and Zuuring, H.R., 2008. Hydrologic calibration and validation of SWAT in a snow-dominated Rocky Mountain watershed, Montana, USA. JAWRA Journal of the American Water Resources Association, 44(6), pp.14111430.

Ajaaj, A. A., Mishra, A. K., and Khan, A. A., 2016. Comparison of BIAS Correction Techniques for GPCC Rainfall Data in Semi-Arid Climate. Stochastic Environmental Research and Risk Assessment, 30(6), pp.1659-1675.

Andres, T.H., 1997. Sampling methods and sensitivity analysis for large parameter sets. Journal of Statistical Computation and Simulation, 57(1-4), pp.77-110.

Andrews, R.N.L., 2006. Managing the Environment, Managing Ourselves. New Haven: Yale University Press.

Arnault, J., Wagner, S., Rummler, T., Fersch, B., Bliefernicht, J., Andresen, S. and Kunstmann, H., 2016. Role of runoff-infiltration partitioning and resolved overland flow on land-atmosphere feedbacks: A case study with the WRF-Hydro coupled modeling system for West Africa. Journal of Hydrometeorology, 17(5), pp.14891516.

Arnell, N. W., 2004. Climate Change and Global Water Resources: SRES Emissions and Socio-economic Scenarios. Global Environmental Change, 14(1), pp.31-52.

Arnold, J. G., Kiniry, J. R., Srinivasan, R., Williams, J. R., Haney, E. B., and Neitsch, S. L., 2013. Soil and Water Assessment Tool: Input/output Documentation. Version 2012. Texas Water Resources Institute. TR-439, 650.

Arnold, J., Srinivasan, R., Muttiah, R., and Williams, J., 1998. Large Area Hydrologic Modeling and Assessment Part I: Model Development. JAWRA Journal of the American Water Resources Association, 34(1), pp.73-89. 
Arnold, J.G., Allen, P.M. and Bernhardt, G., 1993. A comprehensive surfacegroundwater flow model. Journal of Hydrology, 142(1-4), pp.47-69.

Ashraf Vaghefi, S., Abbaspour, K., Faramarzi, M., Srinivasan, R. and Arnold, J., 2017. Modeling Crop Water Productivity Using a Coupled SWAT-MODSIM Model. Water, 9(3), p.157.

Ashraf Vaghefi, S., Mousavi, S., Abbaspour, K., Srinivasan, R., and Arnold, J., 2015. Integration of Hydrologic and Water Allocation Models in Basin-scale Water Resources Management Considering Crop Pattern and Climate Change: Karkheh River Basin in Iran. Regional Environmental Change, 15(3), pp.475-484.

Babar, S. and Ramesh, H., 2015. Streamflow Response to Land Use-Land Cover Change over the Nethravathi River Basin, India. ASCE Journal of Hydrologic Engineering, 20(10), pp.05015002.

Bailey, R. T., Wible, T. C., Arabi, M., Records, R. M., and Ditty, J., 2016. Assessing regional-scale spatio-temporal patterns of groundwater-surface water interactions using a coupled SWAT-MODFLOW model. Hydrological Processes, 30(23), pp.4420-4433.

Bailey, R., Rathjens, H., Bieger, K., Chaubey, I. and Arnold, J., 2017. SWATMOD-Prep: Graphical user interface for preparing coupled SWAT-MODFLOW simulations. JAWRA Journal of the American Water Resources Association, 53(2), pp.400-410.

Baker, T.J. and Miller, S.N., 2013. Using the Soil and Water Assessment Tool (SWAT) to assess land use impact on water resources in an East African watershed. Journal of Hydrology, 486, pp.100-111.

Ballweber, J., 2006. A Comparison of IWRM Frameworks: The United States and South Africa. Journal of Contemporary Water Research and Education, 135(1), pp.74-79.

Bannwarth, M. A., Sangchan, W., Hugenschmidt, C., Lamers, M., Ingwersen, J., Ziegler, A. D., and Streck, T., 2014. Pesticide transport simulation in a tropical catchment by SWAT. Environmental Pollution, 191, pp.70-79. 
Barbosa, S. M., 2008. Quantile Trends in Baltic Sea Level. Geophysical Research Letters, 35(22).

Bayissa, Y., Moges, S., Xuan, Y., Van Andel, S., Maskey, S., and Solomatine, D. et al., 2015. Spatio-Temporal Assessment of Meteorological Drought under the Influence of Varying Record Length: The Case of Upper Blue Nile Basin, Ethiopia. Hydrological Sciences Journal, pp.1-16.

Bell, V., and Moore, R., 2000. The sensitivity of catchment runoff models to rainfall data at different spatial scales. Hydrology and Earth System Sciences, 4(4), pp.653-667.

Best, M.J., Pryor, M., Clark, D.B., Rooney, G.G., Essery, R., Ménard, C.B., Edwards, J.M., Hendry, M.A., Porson, A., Gedney, N. and Mercado, L.M., 2011. The Joint UK Land Environment Simulator (JULES), model description-Part 1: energy and water fluxes. Geoscientific Model Development, 4(3), pp.677-699.

Betrie, G.D., Van Griensven, A., Mohamed, Y.A., Popescu, I., Mynett, A.E. and Hummel, S., 2011. Linking SWAT and SOBEK using open modeling interface (OPENMI) for sediment transport simulation in the Blue Nile River basin. Transactions of the ASABE, 54(5), pp.1749-1757.

Beven, K. and Binley, A., 1992. The future of distributed models: model calibration and uncertainty prediction. Hydrological Processes, 6(3), pp.279-298.

Beven, K. and Freer, J., 2001. Equifinality, data assimilation, and uncertainty estimation in mechanistic modelling of complex environmental systems using the GLUE methodology. Journal of Hydrology, 249(1-4), pp.11-29.

Bhave, A., Conway, D., Dessai, S. and Stainforth, D., 2018. Water Resource Planning Under Future Climate and Socioeconomic Uncertainty in the Cauvery River Basin in Karnataka, India. Water Resources Research.

Biswas, A., 2008. Current Directions: Integrated Water Resources Management - a Second Look. Water International, 33(3), pp.274-278. 
Blasone, R.S., Madsen, H. and Rosbjerg, D., 2008. Uncertainty assessment of integrated distributed hydrological models using GLUE with Markov chain Monte Carlo sampling. Journal of Hydrology, 353(1-2), pp.18-32.

Bocheva, L., Marinova, T., Simeonov, P., and Gospodinov, I., 2009. Variability and Trends of Extreme Precipitation Events over Bulgaria (1961-2005). Atmospheric Research, 93(1-3), pp.490-497.

Breidt, J., 2005. Nonlinear Time Series: Nonparametric and Parametric Methods. Journal of the American Statistical Association, 100(469), 348-349.

Bremnes, J., 2004. Probabilistic Forecasts of Precipitation in Terms of Quantiles using NWP Model Output. Monthly Weather Review, 132(1), pp.338-347.

British Atmospheric Data Centre, 2015. Met Office - MIDAS Land Surface Stations Data. Unpublished raw data.

Bronaugh, D., 2015. R package 'climdex. Pcic': PCIC Implementation of Climdex Routines. Victoria, British Columbia, Canada: Pacific Climate Impact Consortium.

Brown, J. F., Wardlow, B. D., Tadesse, T., Hayes, M. J., and Reed, B. C., 2008. The Vegetation Drought Response Index (VegDRI): A New Integrated Approach for Monitoring Drought Stress in Vegetation. GIScience and Remote Sensing, 45(1), pp.16-46.

Brunner, P., and Simmons, C., 2012. HydroGeoSphere: A Fully Integrated, Physically Based Hydrological Model. Ground Water, 50(2), pp.170-176.

Butts, M., Payne, J., Kristensen, M., and Madsen, H., 2004. An Evaluation of the Impact of Model Structure on Hydrological Modelling Uncertainty for Streamflow Simulation. Journal of Hydrology, 298(1-4), pp.242-266.

Cai, Y. and Reeve, D., 2013. Extreme Value Prediction via a Quantile Function Model. Coastal Engineering, 77, pp.91-98. 
Campolongo, F., Cariboni, J. and Saltelli, A., 2007. An effective screening design for sensitivity analysis of large models. Environmental Modelling \& Software, 22(10), pp.1509-1518.

Campolongo, F., Saltelli, A. and Cariboni, J., 2011. From screening to quantitative sensitivity analysis. A unified approach. Computer Physics Communications, 182(4), pp.978-988.

Cannon, A., 2011. Quantile Regression Neural Networks: Implementation in R and Application to Precipitation Downscaling. Computers and Geosciences, 37(9), pp.1277-1284.

Cannon, A., Sobie, S., and Murdock, T., 2015. Bias Correction of GCM Precipitation by Quantile Mapping: How Well Do Methods Preserve Changes in Quantiles and Extremes? Journal of Climate, 28(17), pp.6938-6959.

Caruso, C., and Quarta, F., 1998. Interpolation methods comparison. Computers and Mathematics with Applications, 35(12), pp.109-126.

Chaplot, V., Saleh, A., and Jaynes, D., 2005. Effect of the Accuracy of Spatial Rainfall Information on the Modeling of Water, Sediment, and $\mathrm{NO}_{3}-\mathrm{N}$ Loads at the Watershed Level. Journal of Hydrology, 312(1-4), pp.223-234.

Chattopadhyay, 2014. Climate Change Impact Assessment on Watershed Hydrology: A Comparison of Three Approaches. American Journal of Engineering and Applied Sciences, 7(1), pp.122-128.

Chattopadhyay, S., and Jha, M. K., 2016. Hydrological Response Due to Projected Climate Variability in Haw River Watershed, North Carolina, USA. Hydrological Sciences Journal, 61(3), pp.495-506.

Chaubey, I., Haan, C., Grunwald, S., and Salisbury, J., 1999. Uncertainty in the model parameters due to spatial variability of rainfall. Journal of Hydrology, 220(1-2), pp.48-61. 
Chen, S.M., Wang, Y.M. and Tsou, I., 2013. Using artificial neural network approach for modelling rainfall-runoff due to typhoon. Journal of Earth System Science, 122(2), pp.399-405.

Cheng, Q., Chen, X., Xu, C., Reinhardt-Imjela, C., and Schulte, A., 2014. Improvement and Comparison of Likelihood Functions for Model Calibration and Parameter Uncertainty Analysis within a Markov Chain Monte Carlo Scheme. Journal of Hydrology, 519, pp.2202-2214.

Cheng, S.J., 2011. The best relationship between lumped hydrograph parameters and urbanized factors. Natural hazards, 56(3), pp.853-867.

Chiang, W.H. and Kinzelbach, W., 1998. Processing MODFLOW: a simulation system for modeling groundwater flow and pollution. Software Instruction Book, Hamburg-Zurich.

Cho, H., and Olivera, F., 2009. Effect of the Spatial Variability of Land Use, Soil Type, and Precipitation on Streamflow in Small Watersheds. JAWRA Journal of the American Water Resources Association, 45(3), pp.673-686.

Chow, T. V., Maidment, R. D., \& Mays, W. L., 1988. Applied hydrology (2010 Ed.). New York, NY: Tata McGraw-Hill.

Chu, M.L., Knouft, J.H., Ghulam, A., Guzman, J.A. and Pan, Z., 2013. Impacts of urbanization on river flow frequency: A controlled experimental modeling-based evaluation approach. Journal of Hydrology, 495, pp.1-12.

Chu, T.W. and Shirmohammadi, A., 2004. Evaluation of the SWAT model's hydrology component in the piedmont physiographic region of Maryland. Transactions of the ASAE, 47(4), p.1057.

Clark, D.B., Mercado, L.M., Sitch, S., Jones, C.D., Gedney, N., Best, M.J., Pryor, M., Rooney, G.G., Essery, R.L.H., Blyth, E. and Boucher, O., 2011. The Joint UK Land Environment Simulator (JULES), model description-Part 2: carbon fluxes and vegetation dynamics. Geoscientific Model Development, 4(3), pp.701-722. 
Cole, S. J., and Moore, R. J., 2008. Hydrological modelling using raingauge- and radarbased estimators of areal rainfall. Journal of Hydrology, 358(3-4), pp.159-181.

Collischonn, B., Collischonn, W., and Tucci, C. E. M., 2008. Daily hydrological modeling in the Amazon basin using TRMM rainfall estimates. Journal of Hydrology, 360(1-4), pp.207-216.

Condon, L., and Maxwell, R., 2013. Implementation of a Linear Optimization Water Allocation Algorithm into a Fully Integrated Physical Hydrology Model. Advances in Water Resources, 60, pp.135-147.

Cukier, R.I., Fortuin, C.M., Shuler, K.E., Petschek, A.G. and Schaibly, J.H., 1973. Study of the sensitivity of coupled reaction systems to uncertainties in rate coefficients. I Theory. The Journal of Chemical Physics, 59(8), pp.3873-3878.

Cullen, A. C., and Frey, H. C., 1999. Probabilistic Techniques in Exposure Assessment: A Handbook for Dealing with Variability and Uncertainty in Models and Inputs. Springer Science and Business Media.

De Schepper, G., Therrien, R., Refsgaard, J., and Hansen, A., 2015. Simulating Coupled Surface and Subsurface Water Flow in a Tile-drained Agricultural Catchment. Journal of Hydrology, 521, pp.374-388.

Dee Valley Water and United Utilities, 2017. River Dee Catchment: Using Data and Evidence to Target Water Quality Measures. Westcountry Rivers Trust for United Utilities, pp.1-16.

DEFRA, 2008. Future Water: The Government's water strategy for England.

DEFRA, 2014. The Impact of Water Abstraction Reform: Public Water Supply Operations. Wallingford: Department for Environment, Food and Rural Affairs/ Flood Risk Management Division.

Delignette-Muller, M. L., and Dutang, C., 2014. fitdistrplus: An R Package for Fitting Distributions. Journal of Statistical Software, 64, pp.1-34. 
Dinpashoh, Y., Mirabbasi, R., Jhajharia, D., Abianeh, H. Z., and Mostafaeipour, A., 2014. Effect of Short-Term and Long-Term Persistence on Identification of Temporal Trends. ASCE Journal of Hydrologic Engineering, 19(3), pp.617-625.

Dirks, K., Hay, J., Stow, C., and Harris, D., 1998. High-resolution studies of rainfall on Norfolk Island. Journal of Hydrology, 208(3-4), pp.187-193.

Donat, M. G., Alexander, L. V., Yang, H., Durre, I., Vose, R., Dunn, R. J. H., Hewitson, B. et al., 2013. Updated Analyses of Temperature and Precipitation Extreme Indices since the Beginning of the Twentieth Century: The HadEX2 Dataset. Journal of Geophysical Research: Atmospheres, 118(5), pp.2098-2118.

Droogers, P., 2009. Climate Change and Hydropower, Impact and Adaptation Costs: Case Study Kenya. Report Future Water 85, 27.

Duffy, C. J., 2004. Semi-Discrete Dynamical Model for Mountain-Front Recharge and Water Balance Estimation, Rio Grande of Southern Colorado and New Mexico. Groundwater Recharge in a Desert Environment: The Southwestern United States, pp.255-271.

Dutta, D., Kim, S., Vaze, J., and Hughes, J., 2015. Streamflow Predictions in Regulated River Systems: Hydrological Non-stationarity versus Anthropogenic Water Use. Proceedings of the International Association of Hydrological Sciences, 371, pp.3542.

Eberhart, R. and Kennedy, J., 1995, October. A new optimizer using particle swarm theory. In Micro Machine and Human Science, 1995. MHS'95., Proceedings of the Sixth International Symposium on (pp. 39-43). IEEE.

Eckhardt, K., 2008. A comparison of baseflow indices, which were calculated with seven different baseflow separation methods. Journal of Hydrology, 352(1-2), pp.168173. 
Eden, J., Widmann, M., Grawe, D., and Rast, S., 2012. Skill, Correction, and Downscaling of GCM-Simulated Precipitation. Journal of Climate, 25(11), pp.3970-3984.

Elsner, J. B., Kossin, J. P., and Jagger, T. H., 2008. The Increasing Intensity of the Strongest Tropical Cyclones. Nature, 455(7209), pp.92-95.

Engle, N. L., Johns, O. R., Lemos, M., and Nelson D. R., 2011. Integrated and Adaptive Management of Water Resources: Tensions, Legacies, and the Next Best Thing. Ecology and Society 16(1).

Environment Agency Wales, 2010. Catchment Flood Management Plan, pp. 1-26. Cardiff.

Fan, L., and Chen, D., 2016. Trends in Extreme Precipitation Indices across China Detected using Quantile Regression. Atmospheric Science Letters, 17(7), pp.400406.

FAO, 2003. Towards Sustainable Agricultural Development in Iraq. The Transition from Relief, Rehabilitation and Reconstruction to Development.

Feidas, H., Makrogiannis, T., and Bora-Senta, E., 2004. Trend Analysis of Air Temperature Time series in Greece and Their Relationship with Circulation Using Surface and Satellite Data: 1955-2001. Theoretical and Applied Climatology, 79(34), pp.185-208.

Fersch, B., Gochis, D.J., Kunstmann, H., Mendicino, G. and Senatore, A., 2014. Book of Abstracts of the 1st European Fully Coupled Atmospheric-Hydrological Modeling and WRF-Hydro Users Workshop. Univ. of Calabria, Rende (CS), Italy. Available at http://cesmma. unical. it/wrf-hydro2104/BookOfAbstracts. pdf.

Ficklin, D., Stewart, I., and Maurer, E., 2013. Climate Change Impacts on Stream flow and Subbasin-Scale Hydrology in the Upper Colorado River Basin. PLoS ONE, 8(8), e71297. 
Friederichs, P., and Hense, A., 2007. Statistical Downscaling of Extreme Precipitation Events using Censored Quantile Regression. Monthly Weather Revision, 135(6), pp.2365-2378.

Fu, J., Qian, W., Lin, X., and Chen, D., 2008. Trends in Graded Precipitation in China from 1961 to 2000. Advances in Atmospheric Sciences, 25(2), pp.267-278.

Fuka, D.R., Walter, M.T., Archibald, J.A., Steenhuis, T.S., Easton, Z.M., Fuka, M.D. and KeepSource, T.R.U.E., 2014. Package 'EcoHydRology’.

Gain, A., Giupponi, C., and Renaud, F., 2012. Climate Change Adaptation and Vulnerability Assessment of Water Resources Systems in Developing Countries: A Generalized Framework and a Feasibility Study in Bangladesh. Water, 4(4), pp.345-366.

Gain, A., Rouillard, J., and Benson, D., 2013. Can Integrated Water Resources Management Increase Adaptive Capacity to Climate Change Adaptation? A Critical Review. Journal of Water Resource and Protection, 05(04), pp.11-20.

Ganguli, P., and Reddy, M. J., 2014. Evaluation of Trends and Multivariate Frequency Analysis of Droughts in Three Meteorological Subdivisions of Western India. International Journal of Climatology, 34(3), pp.911-928.

Garner, G., Hannah, D.M. and Watts, G., 2017. Climate change and water in the UK: Recent scientific evidence for past and future change. Progress in Physical Geography, 41(2), pp.154-170.

Gassman, P. W., Reyes, M. R., Green, C. H., and Arnold, J. G., 2007. The Soil and Water Assessment Tool: historical development, applications, and future research directions. Transactions of the ASABE, 50(4), pp.1211-1250.

Global Water Partnership, 2012. What is IWRM? [Online] Available at: https://globalwaterpartnership.wordpress.com/what-is-iwrm/ [Accessed 15 May 2017]. 
Gochis, D.J., Yu, W. and Yates, D.N., 2013. The WRF-Hydro Model Technical Description and User's Guide, Version 1.0, NCAR Technical Document, 120 pp., NCAR, Boulder, Colorado.

Golmohammadi, G., Rudra, R., Dickinson, T., Goel, P. and Veliz, M., 2017. Predicting the temporal variation of flow contributing areas using SWAT. Journal of Hydrology, 547, pp.375-386.

Green, W.H. and Ampt, G.A., 1911. Studies on Soil Physics. The Journal of Agricultural Science, 4(1), pp.1-24.

Gregersen, J., Gijsbers, P., and Westen, S., 2007. OpenMI: Open Modelling Interface. Journal of Hydroinformatics, 9(3), pp.175-191.

Griffiths, G., Salinger, M., and Leleu, I., 2003. Trends in Extreme Daily Rainfall across the South Pacific and Relationship to the South Pacific Convergence Zone. International Journal of Climatology, 23(8), pp.847-869.

Griggs, D. J., and Noguer, M., 2002. Climate Change 2001: The Scientific Basis. Contribution of Working Group I to the Third Assessment Report of the Intergovernmental Panel on Climate Change. Weather, 57(8), pp.267-269.

Gringorten, I., 1963. A Plotting Rule for Extreme Probability Paper. Journal of Geophysical Research, 68(3), pp.813-814.

Gupta, H.V., Beven, K.J. and Wagener, T., 2006. Model calibration and uncertainty estimation. Encyclopedia of hydrological sciences.

Guzman, J.A., Moriasi, D.N., Gowda, P.H., Steiner, J.L., Starks, P.J., Arnold, J.G. and Srinivasan, R., 2015. A model integration framework for linking SWAT and MODFLOW. Environmental Modelling \& Software, 73, pp.103-116.

Hadded, R., Nouiri, I., Alshihabi, O., Maßmann, J., Huber, M., and Laghouane, A., 2013. A Decision Support System to Manage the Groundwater of the Zeuss Koutine 
Aquifer Using the WEAP-MODFLOW Framework. Water Resources Management, 27(7), pp.1981-2000.

Hao, L., Sun, G., Liu, Y. and Qian, H., 2015. Integrated Modeling of Water Supply and Demand under Management Options and Climate Change Scenarios in Chifeng City, China. JAWRA Journal of the American Water Resources Association, 51(3), pp.655-671.

Hao, Z., and AghaKouchak, A., 2013. Multivariate Standardized Drought Index: A Parametric Multi-Index Model. Advances in Water Resources, 57, pp.12-18.

Hargreaves, G.L., Hargreaves, G.H. and Riley, J.P., 1985. Irrigation water requirements for Senegal River basin. Journal of Irrigation and Drainage Engineering, 111(3), pp.265-275.

Hartmann, A., Wagener, T., Rimmer, A., Lange, J., Brielmann, H. and Weiler, M., 2013. Testing the realism of model structures to identify karst system processes using water quality and quantity signatures. Water Resources Research, 49(6), pp.33453358.

Hassanzadeh, E., Elshorbagy, A., Wheater, H., Gober, P., and Nazemi, A., 2016. Integrating Supply Uncertainties from Stochastic Modeling into Integrated Water Resource Management: Case Study of the Saskatchewan River Basin. ASCE Journal of Water Resources Planning and Management, 142(2), pp.05015006-105015006-12.

Hassing, J., 2009. Integrated Water Resources Management in Action: Dialogue Paper. Unesco.

Helsel, D.R., Hirsch, R.M., 1992. Statistical Methods in Water Resources. Elsevier, New York.

Heuvelmans, G., Muys, B. and Feyen, J., 2006. Regionalisation of the parameters of a hydrological model: Comparison of linear regression models with artificial neural nets. Journal of Hydrology, 319(1-4), pp.245-265. 
Hirsch, R., Slack, J., and Smith, R., 1982. Techniques of Trend Analysis for Monthly Water Quality Data. Water Resources Research, 18(1), pp.107-121.

IPCC, 2007. Climate change, 2007: Climate Change Impacts, Adaptation and Vulnerability. Working Group II Contribution to the Intergovernmental Panel on Climate Change Fourth Assessment Report. Summary for Policymakers, p 23

Jain, S.K. and Singh, V.P., 2003. Water resources systems planning and management (Vol. 51). Elsevier.

Jayakrishnan, R., Srinivasan, R., Santhi, C., and Arnold, J., 2005. Advances in the Application of the SWAT Model for Water Resources Management. Hydrological Processes, 19(3), pp.749-762.

Jin, X., and Sridhar, V., 2011. Impacts of Climate Change on Hydrology and Water Resources in the Boise and Spokane River Basins1. JAWRA Journal of the American Water Resources Association, 48(2), pp.197-220.

Jin, X., Xu, C., Zhang, Q., and Singh, V., 2010. Parameter and Modeling Uncertainty Simulated by GLUE and a Formal Bayesian Method for a Conceptual Hydrological Model. Journal of Hydrology, 383(3-4), pp.147-155.

Johnson, G. L., and Hanson, C. L., 1995. Topographic and atmospheric influences on precipitation variability over a mountainous watershed. Journal of Applied Meteorology, 34(1), pp.68-87.

Joyce, B., Mehta, V., Purkey, D., Dale, L., and Hanemann, M., 2011. Modifying Agricultural Water Management to Adapt to Climate Change in California's Central Valley. Climatic Change, 109(S1), pp.299-316.

Kankam-Yeboah, K., Obuobie, E., Amisigo, B. and Opoku-Ankomah, Y., 2013. Impact of climate change on streamflow in selected river basins in Ghana. Hydrological Sciences Journal, 58(4), pp.773-788. 
Kannan, N., White, S.M., Worrall, F. and Whelan, M.J., 2007. Sensitivity analysis and identification of the best evapotranspiration and runoff options for hydrological modelling in SWAT-2000. Journal of Hydrology, 332(3-4), pp.456-466.

Katirtzidou, M. and Latinopoulos, P., 2017. Allocation of surface and subsurface water resources to competing uses under climate changing conditions: a case study in Halkidiki, Greece. Water Science and Technology: Water Supply, p.ws2017166.

Keller, V., Tanguy, M., Prosdocimi, I., Terry, J., Hitt, O., and Cole, S. et al., 2015. CEHGEAR: $1 \mathrm{~km}$ Resolution Daily and Monthly Areal Rainfall Estimates for the UK for Hydrological and other Applications. Earth System Science Data, 7(1), pp.143155.

Kendall, M. G., 1975. Rank Auto-correlation Methods. Charles Griffin, London.

Kerandi, N., Arnault, J., Laux, P., Wagner, S., Kitheka, J. and Kunstmann, H., 2018. Joint atmospheric-terrestrial water balances for East Africa: a WRF-Hydro case study for the upper Tana River basin. Theoretical and Applied Climatology, 131(3-4), pp.1337-1355.

Kim, N., Chung, I., Won, Y., and Arnold, J., 2008. Development and Application of the Integrated SWAT-MODFLOW Model. Journal of Hydrology, 356(1-2), pp.1-16.

Kim, N.W., Won, Y.S., Lee, J., Lee, J.E. and Jeong, J., 2011. Hydrological impacts of urban imperviousness in White Rock Creek watershed. Transactions of the ASABE, 54(5), pp.1759-1771.

Kirby, J.M., Mainuddin, M., Mpelasoka, F., Ahmad, M.D., Palash, W., Quadir, M.E., Shah-Newaz, S.M. and Hossain, M.M., 2016. The impact of climate change on regional water balances in Bangladesh. Climatic change, 135(3-4), pp.481-491.

Kirchner, J.W., 2006. Getting the right answers for the right reasons: Linking measurements, analyses, and models to advance the science of hydrology. Water Resources Research, 42(3). 
Koenker, R. and Bassett, G, 1978. Regression Quantiles. Econometrica, 46(1), 33.

Koenker, R., 2005. Quantile Regression (No. 38. Cambridge university press.

Koenker, R., Portnoy, S., Ng, P. T., Zeileis, A., Grosjean, P., and Ripley, B. D., 2016. Package 'quantreg'.

Ledoux, H., and Gold, C., 2005. An Efficient Natural Neighbour Interpolation Algorithm for Geoscientific Modelling. Developments in Spatial Data Handling, pp.97-108.

Lenhart, T., Eckhardt, K., Fohrer, N. and Frede, H.G., 2002. Comparison of two different approaches of sensitivity analysis. Physics and Chemistry of the Earth, Parts $A / B / C, 27(9-10)$, pp.645-654.

Li, L., Xu, C. and Engeland, K., 2013. Development and comparison in uncertainty assessment based Bayesian modularization method in hydrological modeling. Journal of Hydrology, 486, pp.384-394.

Li, Q., Li, P., Li, H., and Yu, M., 2015. Drought Assessment using a Multivariate Drought Index in the Luanhe River Basin of Northern China. Stochastic Environmental Research and Risk Assessment, 29(6), pp.1509-1520.

Liu, Y. and Gupta, H.V., 2007. Uncertainty in hydrologic modeling: Toward an integrated data assimilation framework. Water Resources Research, 43(7).

Lloyd, C. D., 2005. Assessing the effect of integrating elevation data into the estimation of monthly precipitation in Great Britain. Journal of Hydrology, 308(1-4), pp.128150.

Looper, J., and Vieux, B., 2011. An assessment of distributed flash flood forecasting accuracy using radar and rain gauge input for a physics-based distributed hydrologic model. Journal of Hydrology, 412-413, pp.114-132.

Loucks, D. P., Van Beek, E., Stedinger, J. R., Dijkman, J. P., and Villars, M. T., 2005. Water Resources Systems Planning and Management: An Introduction to Methods, Models and Applications. Paris: UNESCO. 
Loucks, D., 2008. Water Resource Management Models. The Bridge, 38(3), pp.24-30.

Lu, G. Y., and Wong, D. W., 2008. An adaptive inverse-distance weighting spatial interpolation technique. Computers and Geosciences, 34(9), pp.1044-1055.

Luce, C., and Holden, Z., 2009. Declining Annual Streamflow Distributions in the Pacific Northwest United States, 1948-2006. Geophysical Research Letters, 36(16).

Luo, Y., Arnold, J., Allen, P. and Chen, X., 2012. Baseflow simulation using SWAT model in an inland river basin in Tianshan Mountains, Northwest China. Hydrology and Earth System Sciences, 16(4), pp.1259-1267.

Ly, S., Charles, C., and Degre, A., 2011. Geostatistical interpolation of daily rainfall at catchment scale: the use of several variogram models in the Ourthe and Ambleve catchments, Belgium. Hydrology and Earth System Sciences, 15(7), pp.2259-2274.

Lyne, V. and Hollick, M., 1979, September. Stochastic time-variable rainfall-runoff modelling. In Institute of Engineers Australia National Conference (Vol. 1979, pp. 89-93.

Maganga, F., Kiwasila, H., Juma, I., and Butterworth, J., 2004. Implications of Customary Norms and Laws for Implementing IWRM: Findings from Pangani and Rufiji Basins, Tanzania. Physics and Chemistry of the Earth, Parts A/B/C, 29(1518), pp.1335-1342.

Malagó, A., Bouraoui, F., Vigiak, O., Grizzetti, B. and Pastori, M., 2017. Modelling water and nutrient fluxes in the Danube River Basin with SWAT. Science of the Total Environment, 603-604, pp.196-218.

Mandapaka, P.V., Krajewski, W.F., Mantilla R. and Gupta, V.K., 2009. Dissecting the Effect of Rainfall Variability on the Statistical Structure of Peak Flows. Advances in Water Resources, 32(10), pp.1508-1525.

Mann, H. B., 1945. Nonparametric Tests against Trend. Econometrica: Journal of the Econometric Society, pp.245-259. 
Maraun, D., 2013. Bias Correction, Quantile Mapping, and Downscaling: Revisiting the Inflation Issue. Journal of Climate, 26(6), pp.2137-2143.

Maraun, D., Wetterhall, F., Ireson, A., Chandler, R., Kendon, E., and Widmann, M. et al., 2010. Precipitation Downscaling under Climate Change: Recent Developments to Bridge the Gap between Dynamical Models and the End User. Review of Geophysics, 48(3), pp.1-34.

Marchetto, A., 2015. rkt: Mann-Kendall Test, Seasonal and Regional Kendall Tests. $R$ package. Technology, 40, pp.4066-4073.

Martinez, C., Maleski, J., and Miller, M., 2012. Trends in Precipitation and Temperature in Florida, USA. Journal of Hydrology, 452-453, pp.259-281.

Masih, I., Maskey, S., Uhlenbrook, S. and Smakhtin, V., 2011. Assessing the Impact of Areal Precipitation Input on Streamflow Simulations using the SWAT Model. JAWRA Journal of American Water Resources Association, 47(1), pp.179-195.

Maskey, S., Guinot, V., and Price, R., 2004. Treatment of Precipitation Uncertainty in Rainfall-runoff Modelling: A Fuzzy Set Approach. Advances in Water Resources, 27(9), pp.889-898.

Maurer, E., and Pierce, D., 2014. Bias Correction Can Modify Climate Model Simulated Precipitation Changes without Adverse Effect on the Ensemble Mean. Hydrology and Earth System Sciences, 18(3), pp.915-925.

Mayall, S., 2000. Management of the River Dee System: The 1995/96 Drought Experience. BHS occasional paper, 11, pp.125-132.

Mazvimavi, D., 2010. Investigating Changes over Time of Annual Rainfall in Zimbabwe. Hydrological Earth System Sciences. 14(12), pp.2671-2679.

McCray, K., 2001. American agriculture and ground water: brining issues to the surface. Irrigation Journal, 51(5), pp.27-29. 
McDonald, M.G. and Harbaugh, A.W., 1988. A modular three-dimensional finitedifference ground-water flow model (Vol. 6, p. A1). Reston, VA: US Geological Survey.

McKee, T.B., Doesken, N.J. and Kleist, J., 1993, January. The relationship of drought frequency and duration to time scales. In Proceedings of the 8th Conference on Applied Climatology (Vol. 17, No. 22, pp. 179-183). Boston, MA: American Meteorological Society.

Mehta, V., Aslam, O., Dale, L., Miller, N., and Purkey, D., 2013. Scenario-based Water Resources Planning for Utilities in the Lake Victoria Region. Physics and Chemistry of the Earth, Parts A/B/C, 61-62, pp.22-31.

Meinshausen, M., Smith, S. J., Calvin, K., Daniel, J. S., Kainuma, M. L. T., Lamarque, J. F., Thomson, A. et al., 2011. The RCP Greenhouse Gas Concentrations and Their Extensions from 1765 to 2300. Climatic change, 109(1-2), 213.

Melone, F., Barbetta, S., Diomede, T., Peruccacci, S., Rossi, M., Tessarolo, A. and Verdecchia, M., 2005. Review and selection of hydrological models-Integration of hydrological models and meteorological inputs. Contract, (12).

Miao, C., Su, L., Sun, Q., and Duan, Q., 2016. A Nonstationary Bias-correction Technique to Remove Bias in GCM Simulations. Journal of Geophysical Research: Atmospheres, 121(10), pp.5718-5735.

Miller, N., Dale, L., Brush, C., Vicuna, S., Kadir, T., Dogrul, E. and Chung, F., 2009. Drought Resilience of the California Central Valley Surface-Ground-WaterConveyance System. JAWRA Journal of the American Water Resources Association, 45(4), pp.857-866.

Milzow, C. and Kinzelbach, W., 2010. Accounting for subgrid scale topographic variations in flood propagation modeling using MODFLOW. Water Resources Research, 46(10). 
Mishra, S., 2009. Uncertainty and sensitivity analysis techniques for hydrologic modeling. Journal of Hydroinformatics, 11(3-4), pp.282-296.

Mitchell, B., 1990. Integrated water management: international experiences and perspectives. Integrated water management: international experiences and perspectives.

Modarres, R., and Sarhadi, A., 2009. Rainfall Trends Analysis of Iran in the Last Half of the Twentieth Century. Journal of Geophysical Research: Atmospheres, 114(D3), pp.1-9.

Molle, F., 2009. River- Basin Planning and Management: The Social Life of a Concept. Geoforum, 40(3), pp.484-494.

Montanari, A., 2005. Large sample behaviors of the generalized likelihood uncertainty estimation (GLUE) in assessing the uncertainty of rainfall-runoff simulations. Water Resources Research, 41(8).

Monteith, J.L., 1965. Evaporation and environment. The state and movement of water in living organisms. Symposium of the society of experimental biology, Vol. 19 (pp. 205-234).

Moore, R., 2007. The PDM Rainfall-Runoff Model. Hydrological Earth System Sciences, 11(1), pp.483-499.

Morris, M.D., 1991. Factorial sampling plans for preliminary computational experiments. Technometrics, 33(2), pp.161-174.

Mostert, E., Craps, M., and Pahl-Wostl, C., 2008. Social Learning: The Key to Integrated Water Resources Management? Water International, 33(3), pp.293-304.

Mu, Q., Zhao, M., Kimball, J. S., McDowell, N. G., and Running, S. W., 2013. A Remotely Sensed Global Terrestrial Drought Severity Index. Bulletin of the American Meteorological Society, 94(1), pp.83-98. 
Mulwafu, W., and Msosa, H., 2005. IWRM and Poverty Reduction in Malawi: A SocioEconomic Analysis. Physics and Chemistry of the Earth, Parts A/B/C, 30(11-16), pp.961-967.

Naabil, E., Lamptey, B.L., Arnault, J., Olufayo, A. and Kunstmann, H., 2017. Water resources management using the WRF-Hydro modelling system: Case-study of the Tono dam in West Africa. Journal of Hydrology: Regional Studies, 12, pp.196-209.

Nalbantis, I. and Tsakiris, G., 2008. Assessment of Hydrological Drought Revisited. Water Resources Management, 23(5), pp.881-897.

Nalder, I. A., and Wein, R. W., 1998. Spatial interpolation of climatic normals: test of a new method in the Canadian boreal forest. Agricultural and Forest Meteorology, 92(4), pp.211-225.

Nash, J. E., and Sutcliffe, J. V., 1970. River Flow Forecasting through Conceptual Models Part I-A discussion of principles. Journal of Hydrology, 10(3), pp.282-290.

Nathan, R.J. and McMahon, T.A., 1990. Evaluation of automated techniques for base flow and recession analyses. Water Resources Research, 26(7), pp.1465-1473.

Natural Resources Wales and Environment Agency, 2014. Dee River Basin District Consultation on the draft Flood Risk Management Plan (pp. 1-65). Cardiff: Natural Resources Wales and Environment Agency.

Natural Resources Wales, 2009. River Basin Management Plan Dee River Basin District. Cardiff: Natural Resources Wales.

Natural Resources Wales, 2015. The Dee Regulation Scheme. Cardiff: Natural Resources Wales.

Neitsch, S. L., Arnold, J. G., Kiniry, J. R., and Williams, J. R., 2011. Soil and Water Assessment Tool Theoretical Documentation Version 2009. Texas Water Resources Institute. 
New, M., Todd, M., Hulme, M. and Jones, P., 2001. Precipitation measurements and trends in the twentieth century. International Journal of Climatology, 21(15), pp.1889-1922.

Niswonger, R.G., Panday, S. and Ibaraki, M., 2011. MODFLOW-NWT, a Newton formulation for MODFLOW-2005 (No. 6-A37). US Geological Survey.

Norton, J., 2015. An introduction to sensitivity assessment of simulation models. Environmental Modelling \& Software, 69, pp.166-174.

Palmer, W. C., 1965. Meteorological Drought (Vol. 30). Washington, DC: US Department of Commerce, Weather Bureau.

Patel, D.P. and Srivastava, P.K., 2013. Flood hazards mitigation analysis using remote sensing and GIS: correspondence with town planning scheme. Water Resources Management, 27(7), pp.2353-2368.

Patel, D.P. and Srivastava, P.K., 2014. Application of geo-spatial technique for flood inundation mapping of low lying areas. In Remote Sensing Applications in Environmental Research (pp. 113-130). Springer, Cham.

Paulo, A., Ferreira, E., Coelho, C., and Pereira, L., 2005. Drought Class Transition Analysis through Markov and Loglinear Models, an Approach to Early Warning. Agricultural Water Management, 77(1-3), pp.59-81.

Paulo, A., Rosa, R., and Pereira, L., 2012. Climate Trends and Behaviour of Drought Indices Based on Precipitation and Evapotranspiration in Portugal. Natural Hazards and Earth System Science, 12(5), pp.1481-1491.

Perera, B., James, B., and Kularathna, M., 2005. Computer Software Tool REALM for Sustainable Water Allocation and Management. Journal of Environmental Management, 77(4), pp.291-300. 
Philandras, C. M., Nastos, P. T., Kapsomenakis, J., Douvis, K. C., Tselioudis, G., and Zerefos, C. S., 2011. Long Term Precipitation Trends and Variability within the Mediterranean Region. Natural Hazards and Earth System Sciences, 11(12), 3235.

Pianosi, F. and Wagener, T., 2015. A simple and efficient method for global sensitivity analysis based on cumulative distribution functions. Environmental Modelling \& Software, 67, pp.1-11.

Pianosi, F., Sarrazin, F. and Wagener, T., 2015. A Matlab toolbox for global sensitivity analysis. Environmental Modelling \& Software, 70, pp.80-85.

Piao, S., Friedlingstein, P., Ciais, P., de Noblet-Ducoudré, N., Labat, D., and Zaehle, S., 2007. Changes in Climate and Land Use Have a Larger Direct Impact than Rising $\mathrm{CO}_{2}$ on Global River Runoff Trends. Proceedings of the National Academy of Sciences, 104(39), pp.15242-15247.

Piccarreta, M., Capolongo, D., and Boenzi, F., 2004. Trend Analysis of Precipitation and Drought in Basilicata from 1923 to 2000 within a Southern Italy Context. International Journal of Climatology, 24(7), pp.907-922.

Playán, E. and Mateos, L., 2006. Modernization and optimization of irrigation systems to increase water productivity. Agricultural Water Management, 80(1-3), pp.100-116.

Povoa, L. V., Nery, J. T., and Povoa, M. L. V., 2016. Package 'precintcon'.

Powell, E. and Keim, B., 2015. Trends in Daily Temperature and Precipitation Extremes for the Southeastern United States: 1948-2012. Journal of Climate, 28(4), pp.15921612.

Priestley, C.H.B. and Taylor, R.J., 1972. On the assessment of surface heat flux and evaporation using large-scale parameters. Monthly Weather Review, 100(2), pp.8192.

Qu, Y., and Duffy, C., 2007. A Semi-Discrete Finite Volume Formulation for MultiProcess Watershed Simulation. Water Resources Research, 43(8), pp.1-18. 
Ratto, M., Tarantola, S. and Saltelli, A., 2001. Sensitivity analysis in model calibration: GSA-GLUE approach. Computer Physics Communications, 136(3), pp.212-224.

Ritchie, J.T., 1972. Model for predicting evaporation from a row crop with incomplete cover. Water Resources Research, 8(5), pp.1204-1213.

Rodriguez, L., Cello, P., Vionnet, C., and Goodrich, D., 2008. Fully Conservative Coupling of HEC-RAS with MODFLOW to Simulate Stream-Aquifer Interactions in a Drainage Basin. Journal of Hydrology, 353(1-2), pp.129-142.

Rostamian, R., Jaleh, A., Afyuni, M., Mousavi, S.F., Heidarpour, M., Jalalian, A. and Abbaspour, K.C., 2008. Application of a SWAT model for estimating runoff and sediment in two mountainous basins in central Iran. Hydrological Sciences Journal, 53(5), pp.977-988.

Ryu, J., Cho, J., Kim, I.J., Mun, Y., Moon, J.P., Kim, N.W.,Kim, S.J., Kong, D.S. and Lim, K.J., 2011. Enhancement of SWAT-REMM to simulate reduction of total nitrogen with riparian buffer. Transactions of the ASABE, 54(5), pp.1791-1798.

Sahoo, G.B., Ray, C. and De Carlo, E.H., 2006. Calibration and validation of a physically distributed hydrological model, MIKE SHE, to predict streamflow at high frequency in a flashy mountainous Hawaii stream. Journal of Hydrology, 327(1-2), pp.94-109.

Saltelli, A. and Tarantola, S., 2002. On the relative importance of input factors in mathematical models: safety assessment for nuclear waste disposal. Journal of the American Statistical Association, 97(459), pp.702-709.

Saltelli, A., Ratto, M., Andres, T., Campolongo, F., Cariboni, J., Gatelli, D., Saisana, M. and Tarantola, S., 2008. Global sensitivity analysis: the primer. John Wiley \& Sons.

Santhi, C., Kannan, N., Arnold, J., and Di Luzio, M., 2008. Spatial Calibration and Temporal Validation of Flow for Regional Scale Hydrologic Modeling1. JAWRA Journal of the American Water Resources Association, 44(4), pp.829-846. 
Santos, C., 2014. Recent Changes in Temperature and Precipitation Extremes in an Ecological Reserve in Federal District, Brazil. Revista Brasileira De Meteorologia, 29(1), pp.13-20.

Sarrazin, F., Pianosi, F. and Wagener, T., 2016. Global sensitivity analysis of environmental models: convergence and validation. Environmental Modelling \& Software, 79, pp.135-152.

Savenije, H., and Van der Zaag, P., 2008. Integrated Water Resources Management: Concepts and Issues. Physics and Chemistry of the Earth, Parts A/B/C, 33(5), pp.290-297.

Sayemuzzaman, M., and Jha, M., 2014. Seasonal and Annual Precipitation Time Series Trend Analysis in North Carolina, United States. Atmospheric Research, 137, pp.183-194.

Scherberg, J., Baker, T., Selker, J., and Henry, R., 2014. Design of Managed Aquifer Recharge for Agricultural and Ecological Water Supply Assessed Through Numerical Modeling. Water Resources Management, 28(14), pp.4971-4984.

Schneider, C., Laizé, C. L. R., Acreman, M. C., and Florke, M., 2013. How will Climate Change Modify River Flow Regimes in Europe? Hydrology and Earth System Sciences, 17(1), pp.325-339.

Schoups, G., Addams, C.L., Minjares, J.L. and Gorelick, S.M., 2006. Sustainable conjunctive water management in irrigated agriculture: Model formulation and application to the Yaqui Valley, Mexico. Water Resources Research, 42(10).

Schuurmans, J.M. and Bierkens, M.F.P., 2007. Effect of Spatial Distribution of Daily Rainfall on Interior Catchment Response of a Distributed Hydrological Model. Hydrological Earth System Sciences, 11(2), pp.677-693.

Segond, M.L., Wheater, H.S. and Onof, C., 2007. The Significance of Spatial Rainfall Representation for Flood Runoff Estimation: A Numerical Evaluation Based on the Lee Catchment, UK. Journal of Hydrology, 347(1-2), pp.116-131. 
Sen, P., 1968. Estimates of the Regression Coefficient Based on Kendall's Tau. Journal of the American Statistical Association, 63(324), pp.1379-1389.

Senatore, A., Mendicino, G., Gochis, D.J., Yu, W., Yates, D.N. and Kunstmann, H., 2015. Fully coupled atmosphere-hydrology simulations for the central Mediterranean: Impact of enhanced hydrological parameterization for short and long time scales. Journal of Advances in Modeling Earth Systems, 7(4), pp.16931715.

Shafer, B. A., and Dezman, L. E., 1982, April. Development of a Surface Water Supply Index (SWSI) to Assess the Severity of Drought Conditions in Snowpack Runoff Areas. In Proceedings of the Western Snow Conference (Vol. 50), pp. 164-175. Colorado State University Fort Collins CO.

Shah, S., O'Connell, P., and Hosking, J., 1996a. Modelling the effects of spatial variability in rainfall on catchment response. 1. Formulation and calibration of a stochastic rainfall field model. Journal of Hydrology, 175(1-4), pp.67-88.

Shah, S., O'Connell, P., and Hosking, J., 1996b. Modelling the effects of spatial variability in rainfall on catchment response. 2. Experiments with distributed and lumped models. Journal of Hydrology, 175(1-4), pp.89-111.

Sheer, D.P., 1981. Assuring Water Supply for the Washington Metropolitan Area-25 Years of Progress. Pp. 39-66 in a 1980s View of Water Management in the Potomac River Basin. Report of the Committee on Governmental Affairs, U.S. Congress, Senate, 97th Congress, 2d Session, November 12. Washington, D.C.: U.S. Government Printing Office.

Shen, Z., Chen, L., and Chen, T., 2012. Analysis of Parameter Uncertainty in Hydrological and Sediment Modeling using GLUE Method: A Case Study of SWAT Model Applied to Three Gorges Reservoir Region, China. Hydrological Earth System Sciences, 16(1), pp.121-132. 
Shi, X., and Xu, X., 2008. Interdecadal Trend Turning of Global Terrestrial Temperature and Precipitation during 1951-2002. Progress in Natural Science, 18(11), pp.13831393.

Sieber, J. and Purkey, D., 2011. WEAP Water Evaluation and Planning System: User Guide. Somerville, MA: Stockholm Environment Institute, US Centre.

Sieber, J., 2018. WEAP: Water Evaluation and Planning System. [Online] Weap21.org. Available at: http://www.weap21.org/index.asp?action=201 [Accessed 18 Feb. $2018]$.

Sin, G., Gernaey, K.V., Neumann, M.B., van Loosdrecht, M.C. and Gujer, W., 2011. Global sensitivity analysis in wastewater treatment plant model applications: prioritizing sources of uncertainty. Water Research, 45(2), pp.639-651.

Singh, R., Wagener, T., Crane, R., Mann, M.E. and Ning, L., 2014. A vulnerability driven approach to identify adverse climate and land use change combinations for critical hydrologic indicator thresholds: Application to a watershed in Pennsylvania, USA. Water Resources Research, 50(4), pp.3409-3427.

Singh, V. P., 1997. Effect of spatial and temporal variability in rainfall and watershed characteristics on stream flow hydrograph. Hydrological Processes, 11(12), pp.1649-1669.

Singh, V.P. ed., 1995. Computer models of watershed hydrology (Vol. 1130). Highlands Ranch, CO: Water Resources Publications.

Solomon, S. ed., 2007. Climate change 2007-the physical science basis: Working group I contribution to the fourth assessment report of the IPCC (Vol. 4). Cambridge university press.

Sonali, P., and Nagesh Kumar, D., 2013. Review of Trend Detection Methods and Their Application to Detect Temperature Changes in India. Journal of Hydrology, 476, pp.212-227. 
Song, X., Zhang, J., AghaKouchak, A., Roy, S., Xuan, Y., and Wang, G. et al., 2014. Rapid Urbanization and Changes in Spatiotemporal Characteristics of Precipitation in Beijing Metropolitan Area. Journal of Geophysical Research: Atmospheres, 119(19), pp.11, 250-11,271.

Song, X., Zhang, J., Zhan, C., Xuan, Y., Ye, M. and Xu, C., 2015. Global sensitivity analysis in hydrological modeling: Review of concepts, methods, theoretical framework, and applications. Journal of Hydrology, 523, pp.739-757.

Srivastava, P., McNair, J.N. and Johnson, T.E., 2006. Comparison of process-based and artificial neural network approaches for streamflow modeling in an agricultural watershed. JAWRA Journal of the American Water Resources Association, 42(3), pp.545-563.

Srivastava, P.K., Han, D., Ramirez, M.A.R. and Islam, T., 2013a. Appraisal of SMOS soil moisture at a catchment scale in a temperate maritime climate. Journal of Hydrology, 498, pp.292-304.

Srivastava, P.K., Han, D., Ramirez, M.R. and Islam, T., 2013b. Machine learning techniques for downscaling SMOS satellite soil moisture using MODIS land surface temperature for hydrological application. Water Resources Management, 27(8), pp.3127-3144.

Starks, P. J., and Moriasi, D. N., 2009. Spatial Resolution Effect of Precipitation Data on SWAT Calibration and Performance: Implications for CEAP. Transactions of the ASABE, 52(4), pp.1171-1180.

Strayer, D.L., Ewing, H.A. and Bigelow, S., 2003. What kind of spatial and temporal details are required in models of heterogeneous systems? Oikos, 102(3), pp.654662.

Tabari, H., Somee, B., and Zadeh, M., 2011. Testing for Long-Term Trends in Climatic Variables in Iran. Atmospheric Research, 100(1), pp.132-140. 
Tallaksen, L.M., 1995. A review of baseflow recession analysis. Journal of Hydrology, 165(1-4), pp.349-370.

Tan, K.S., Chiew, F.H.S., Grayson, R.B., Scanlon, P.J. and Siriwardena, L., 2005, December. Calibration of a daily rainfall-runoff model to estimate high daily flows. In MODSIM 2005 International Congress on Modelling and Simulation, Melbourne.

Tang, T., Reed, P., Wagener, T. and Van Werkhoven, K., 2007. Comparing sensitivity analysis methods to advance lumped watershed model identification and evaluation. Hydrology and Earth System Sciences Discussions, 3(6), pp.3333-3395.

Tang, Y., Shangyang, L., Xuan, Y., 2009. Integrated Modelling of Flood Forecasting and Multi-reservoir-based Operation in Yellow River Basin, Proceeding of 4th International Yellow River Forum.

Tareghian, R., and Rasmussen, P., 2012. Analysis of Arctic and Antarctic Sea Ice Extent using Quantile Regression. International Journal of Climatology, 33(5), pp.10791086.

Tareghian, R., and Rasmussen, P., 2013. Statistical Downscaling of Precipitation using Quantile Regression. Journal of Hydrology, 487, pp.122-135.

Taylor, R.G., Scanlon, B., Döll, P., Rodell, M., Van Beek, R., Wada, Y., Longuevergne, L., Leblanc, M., Famiglietti, J.S., Edmunds, M. and Konikow, L., 2013. Ground water and climate change. Nature Climate Change, 3(4), p.322.

Teegavarapu, R. S., and Chandramouli, V., 2005. Improved weighting methods, deterministic and stochastic data-driven models for estimation of missing precipitation records. Journal of Hydrology, 312(1-4), pp.191-206.

Tegegne, G., Hailu, I., and Aranganathan, S., 2013. Evaluation of Operation of Lake Tana Reservoir Future Water Use under Emerging Scenario with and without Climate Change Impacts, Upper Blue Nile. International Journal of Computers and Technology, 4(2), pp.654-663. 
Tetzlaff, D., and Uhlenbrook, U., 2005. Effects of Spatial Variability of Precipitation for Process-orientated Hydrological Modelling: Results from Two Nested Catchments. Hydrology and Earth System Sciences Discussions, 2(1), pp.119-154.

Theil, H., 1950. A Rank-Invariant Method of Linear and Polynomial Regression Analysis, 3; Confidence Regions for the Parameters of Polynomial Regression Equations. Stichting Mathematisch Centrum. Statistische Afdeling, (SP 5a/50/R), pp.1-16.

Tobin, K. J., and Bennett, M. E., 2009. Using SWAT to Model Streamflow in Two River Basins with Ground and Satellite Precipitation Data. JAWRA Journal of the American Water Resources Association, 45(1), pp.253-271.

Todd, D. K. and Mays, L. W., 2005. Groundwater Hydrology, Third Edition, John Willey and Sons, Inc. p. 636. USA.

Tomassini, L. and Jacob, D., 2009. Spatial Analysis of Trends in Extreme Precipitation Events in High-resolution Climate Model Results and Observations for Germany. Journal of Geophysical Research, 114(D12).

Trenberth, K.E., 2011. Changes in precipitation with climate change. Climate Research, 47(1/2), pp.123-138.

Tung, Y. K., 2005. Uncertainty and reliability analysis. In Water Resources Systems Management Tools, Chapter 2, Mays LW (ed). McGraw-Hill: New York, 2.1-2.61.

Uhlenbrook, S. and Sieber, A., 2005. On the value of experimental data to reduce the prediction uncertainty of a process-oriented catchment model. Environmental Modelling \& Software, 20(1), pp.19-32.USDA-SCS, 1972. National engineering handbook, hydrology, section 4. US Department of Agriculture, Washington, DC.

Van Griensven, A. and Meixner, T., 2007. A global and efficient multi-objective autocalibration and uncertainty estimation method for water quality catchment models. Journal of Hydroinformatics, 9(4), pp.277-291. 
Van Werkhoven, K., Wagener, T., Reed, P. and Tang, Y., 2008. Characterization of watershed model behavior across a hydroclimatic gradient. Water Resources Research, 44(1).

Vanrolleghem, P.A., Insel, G., Petersen, B., Sin, G., De Pauw, D., Nopens, I., Dovermann, H., Weijers, S. and Gernaey, K., 2003. A comprehensive model calibration procedure for activated sludge models. Proceedings of the Water Environment Federation, 2003(9), pp.210-237.

Vanuytrecht, E., Raes, D. and Willems, P., 2014. Global sensitivity analysis of yield output from the water productivity model. Environmental Modelling \& Software, 51, pp.323-332.

Verdecchia, M., Coppola, E., Tomassetti, B., and Visconti, G., 2009. Cetemps Hydrological Model (CHYM), a Distributed Grid-Based Model Assimilating Different Rainfall Data Sources. In Hydrological Modelling and the Water Cycle (pp. 165-201). Springer Berlin Heidelberg.

Vigiak, O., Malagó, A., Bouraoui, F., Vanmaercke, M., Obreja, F., Poesen, J., Habersack, H., Fehér, J. and Grošelj, S., 2017. Modelling sediment fluxes in the Danube River Basin with SWAT. Science of the Total Environment, 599-600, pp.992-1012.

Villarini, G., Smith, J., Napolitano, F., and Baeck, M., 2011. Hydro-meteorological Analysis of the December 2008 Flood in Rome. Hydrological Sciences Journal, 56(7), pp.1150-1165.

Vrugt, J.A., Ter Braak, C.J., Clark, M.P., Hyman, J.M. and Robinson, B.A., 2008. Treatment of input uncertainty in hydrologic modeling: Doing hydrology backward with Markov chain Monte Carlo simulation. Water Resources Research, 44(12).

Wagener, T. and Kollat, J., 2007. Numerical and visual evaluation of hydrological and environmental models using the Monte Carlo analysis toolbox. Environmental Modelling \& Software, 22(7), pp.1021-1033. 
Wagener, T., McIntyre, N., Lees, M.J., Wheater, H.S. and Gupta, H.V., 2003. Towards reduced uncertainty in conceptual rainfall-runoff modelling: Dynamic identifiability analysis. Hydrological Processes, 17(2), pp.455-476.

Wang, G., Jager, H., Baskaran, L., Baker, T. and Brandt, C., 2016. SWAT Modeling of Water Quantity and Quality in the Tennessee River Basin: Spatiotemporal Calibration and Validation. Hydrology and Earth System Sciences Discussions, pp.1-33.

Waterwise (2012). Water - The Facts Why do we need to think about water? [online] Broads-authority.gov.uk. Available at: http://www.broadsauthority.gov.uk/looking-after/managing-land-and-water/conservationpublications-and-reports/water-conservation-reports/28.-Water-Factsheet-2012.pdf [Accessed 6 Aug. 2018].

Weerts, A., Winsemius, H., and Verkade, J., 2010. Estimation of Predictive Hydrological Uncertainty Using Quantile Regression: Examples from the National Flood Forecasting System (England and Wales). Hydrology and Earth System Sciences Discussions, 7(4), pp.5547-5575.

Wheeler, T. and Von Braun, J., 2013. Climate change impacts on global food security. Science, 341(6145), pp.508-513.

Wijesekara, G.N., Gupta, A., Valeo, C., Hasbani, J.G., Qiao, Y., Delaney, P. and Marceau, D.J., 2012. Assessing the impact of future land-use changes on hydrological processes in the Elbow River watershed in southern Alberta, Canada. Journal of hydrology, 412, pp.220-232.Wilhite, D.A. and Glantz, M.H., 1985. Understanding the Drought Phenomenon: The Role of Definitions. Water International 10(3), pp.111-120.

Wu, K. and Johnston, C.A., 2007. Hydrologic response to climatic variability in a Great Lakes Watershed: A case study with the SWAT model. Journal of Hydrology, 337(1-2), pp.187-199. 
Wurbs, R. A., 1994. Computer Models for Water Resources Planning and Management (No. IWR-94-NDS-7). Arm Engineer Institute for Water Resources Fort Belvoir VA.

Xu, Z., Takeuchi, K., Ishidaira, H., and Li, J., 2005. Long-Term Trend Analysis for Precipitation in Asian Pacific FRIEND River Basins. Hydrological Processes, 19(18), pp.3517-3532.

Xue, C., Chen, B. and Wu, H., 2014. Parameter uncertainty analysis of surface flow and sediment yield in the Huolin Basin, China. Journal of Hydrologic Engineering, 19(6), pp.1224-1236.

Yang, J., 2011. Convergence and uncertainty analyses in Monte-Carlo based sensitivity analysis. Environmental Modelling \& Software, 26(4), pp.444-457.

Yang, J., Reichert, P., Abbaspour, K.C., Xia, J. and Yang, H., 2008. Comparing uncertainty analysis techniques for a SWAT application to the Chaohe Basin in China. Journal of Hydrology, 358(1-2), pp.1-23.

Yates, D., Sieber, J., Purkey, D., and Huber-Lee, A., 2005. WEAP21- A Demand, Priority, and Preference-Driven Water Planning Model. Water International, 30(4), pp.487-500.

Yatheendradas, S., Wagener, T., Gupta, H., Unkrich, C., Goodrich, D., Schaffner, M. and Stewart, A., 2008. Understanding uncertainty in distributed flash flood forecasting for semiarid regions. Water Resources Research, 44(5).

Yoon, Y., Beighley, E., Lee, H., Pavelsky, T., and Allen, G., 2016. Estimating Flood Discharges in Reservoir-Regulated River Basins by Integrating Synthetic SWOT Satellite Observations and Hydrologic Modelling. ASCE Journal of Hydrologic Engineering, 21(4), pp.05015030.

You, Q., Kang, S., Aguilar, E., Pepin, N., Flügel, W. A., Yan, Y., Huang, J. et al., 2011. Changes in Daily Climate Extremes in China and Their Connection to the Large 
Scale Atmospheric Circulation during 1961-2003. Climate Dynamics, 36(11-12), pp.2399-2417.

Zagona, E., Fulp, T., Shane, R., Magee, T., and Goranflo, H., 2001. RIVERWARE: A Generalized Tool for Complex Reservoir System Modeling 1. JAWRA Journal of the American Water Resources Association, 37(4), pp.913-929.

Zhai, P., Zhang, X., Wan, H., and Pan, X., 2005. Trends in Total Precipitation and Frequency of Daily Precipitation Extremes over China. Journal of Climate, 18(7), pp.1096-1108.

Zhang, Y., Cai, W., Chen, Q., Yao, Y., and Liu, K., 2015. Analysis of Changes in Precipitation and Drought in Aksu River Basin, Northwest China. Advances in Meteorology, 2015.

Zhao, P., Jones, P., Cao, L., Yan, Z., Zha, S., and Zhu, Y. et al., 2014. Trend of Surface Air Temperature in Eastern China and Associated Large-Scale Climate Variability over the Last 100 Years. Journal of Climate, 27(12), pp.4693-4703.

Zhu, D., Xuan, Y., and Cluckie, I., 2014. Hydrological Appraisal of Operational Weather Radar Rainfall Estimates in the Context of Different Modelling Structures. Hydrology and Earth System Sciences, 18(1), pp.257-272. 